\title{
Lewis acid catalysed allylboration: Discovery, optimization, and application to the formation of stereogenic quaternary carbon centres
}

\author{
Jason W. J. Kennedy and Dennis G. Hall* \\ Gunning-Lemieux Chemistry Centre, University of Alberta \\ Edmonton, Alberta, Canada, T6G 2G2 \\ dennis.hall@ualberta.ca
}

\section{Supporting Information}

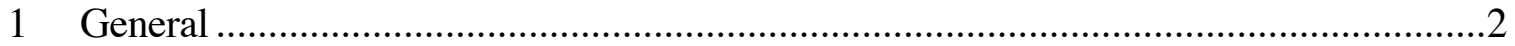

2 X-ray crystallography experimental details ....................................................

3 Stereochemical Assignments.............................................................................

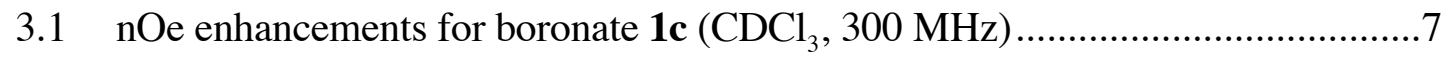

3.2 nOe enhancements for lactones $\mathbf{2 i}$ and $\mathbf{2} \mathbf{j}\left(\mathrm{CDCl}_{3}, 300 \mathrm{MHz}\right)$..........................

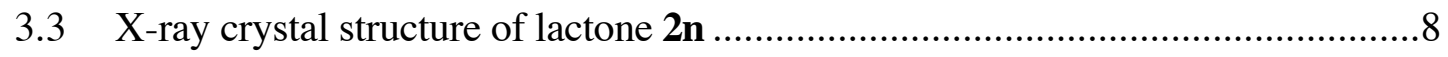

3.4 X-ray crystal structure of lactone $\mathbf{2 0}$

3.5 X-ray crystal structure of $\square$-methyl lactone 27c

4 Preparation of 2-carboxyester 3,3-disubstituted allylboronates 1 ..............................

4.1 Allylboronates 1 from 3 equivalents of electrophile 5a.................................9

4.2 Allylboronates 1 from 2 equivalents of electrophile 5a.................................16

5 Thermal reactions of 2-carboxyester 3,3-disubstituted allylboronates 1 .....................17

5.1 Exo-methylene butyrolactones 2 from diastereoselective allylborations with 1 ...17

5.2 Enantioselective allylborations - Single auxiliary approach .............................24

5.3 Enantioselective allylborations - Dual auxiliary approach.................................39

6 Lewis acid catalysed allylborations with 2-carboxyester 3,3-disubstituted allylboronates

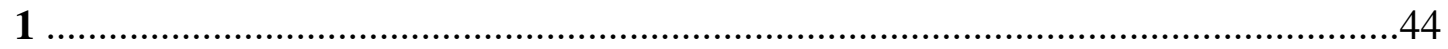

6.1 General procedure for NMR catalyst search ............................................44

6.2 Optimization and control experiments for Lewis acid catalysed allylboration.....46

$6.3{ }^{1} \mathrm{H}$ and ${ }^{11} \mathrm{~B}$ NMR spectra of mixtures of allylboronate $1 \mathrm{c}$ and $\mathrm{Sc}(\mathrm{OTf})_{3} \ldots \ldots \ldots . . .51$

6.4 Catalysed allylborations with aromatic aldehydes........................................54

6.5 Catalysed allylborations with aliphatic aldehydes .......................................60

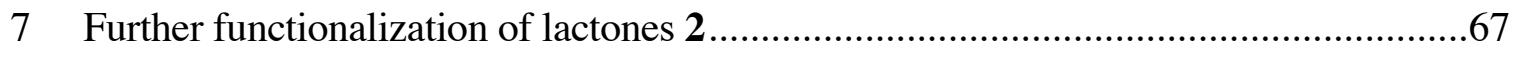


7.1 -Methylbutyrolactones 27 via hydrogenation of the exocyclic double bond.....67

7.2 Reduction of butyrolactone $\mathbf{2 7}$ a to acyclic diol $\mathbf{2 8}$.

$7.3\left(2 R^{*}, 3 R^{*}, 4 S^{*}\right)$-3-Ethyl-2,3-dimethyltridecane-1,4-diol 28

8 NMR spectra of 2-carboxyester-3,3-disubstituted allylboronates $\mathbf{1}$...........................70

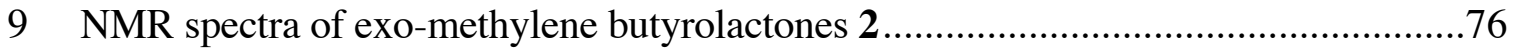

10 NMR spectra of selected single auxiliary chiral alkynoates $\mathbf{1 0}$ and allylboronates $\mathbf{1 1 . . 8 8}$

11 NMR spectra of butyrolactones 27 and diol 28 .92

\section{General}

Experimental details for some compounds have already been published, ${ }^{1}$ but are included here for the convenience of the reader. ${ }^{1} \mathrm{H}$ and ${ }^{13} \mathrm{C}$ NMR spectra for new compounds are included at the end of this section - spectra for other compounds can be found in these previous publications. Methyl 2-heptynoate 3d, ${ }^{2}$ methyl 4-(tertbutyldiphenylsilyloxy)-2-butynoate $\mathbf{3 e},^{3,4}$ methyl 5-(tert-butyldiphenylsilyloxy)-2pentynoate $\mathbf{3 f},{ }^{4}$ methyl 3-phenylpropynoate $\mathbf{3 g},{ }^{2}$ and pinacol iodomethaneboronate $\mathbf{5} \mathbf{a}^{5}$ were prepared by literature procedures. All aldehydes were purified by Kugelrohr distillation prior to use. 8-Arylmenthols $\mathbf{9 f - h}$ were made according to published procedures; ${ }^{6}$ arylmenthol $9 \mathbf{i}$ was prepared in analogy to these procedures. Bornanediol 14 was prepared by Naheed Rajabali according to a literature procedure. ${ }^{7}$ 3-(tert-Butyldiphenylsilyloxy)propanal 12, ${ }^{8}$ diisopropyl bromomethaneboronate $\mathbf{1 6} \mathbf{a}^{9}$ and diisopropyl iodomethaneboronate $\mathbf{1 6} \mathbf{b}^{10}$ were prepared via literature procedures. $(R)$-Pantolactone 9a, $l$-borneol 9b, $(R)$-1-phenylethanol 9c, (-)-menthol 9d, and

(1) (a) Kennedy, J. W. J.; Hall, D. G. J. Am. Chem. Soc. 2002, 124, 898-899; (b) Kennedy, J. W. J.; Hall, D. G. J. Am. Chem. Soc. 2002, 124, 11586-11587.

(2) Hall, D. G.; Ph. D. Thesis, Université de Sherbrooke, 1995, p. 257.

(3) Zacharie, B.; Gagnon, L.; Attardo, G.; Connolly, T. P.; St-Denis, Y.; Penney, C. L. J. Med. Chem. 1997, 40, 2883-2894.

(4) (a) Nagaoka, H.; Kishi, Y. Tetrahedron, 1981, 37, 3873; (b) Hall, D. G.; Ph. D. Thesis, Université de Sherbrooke, 1995, p. 257.

(5) Phillion, D. P.; Neubauer, R.; Andrew, S.S. J. Org. Chem 1986, 51, 1610-1612.

(6) Yang, D. Xu, M.; Bian, M.-Y. Org. Lett. 2001, 3, 111-114. See also (a) Ort. O. Org. Synth. 1987, 65, 203-213; (b) Potin, D.; Dumas, F. Synth. Commun. 1990, 20, 2805-2813.

(7) Herold, T.; Schrott, U.; Hoffmann, R. W.; Schnelle, G.; Ladner, W.; Steinbach, K. Chem. Ber. 1981, 114, 359-374.

(8) Ndibwami, A.; Lamothe, S.; Guay, D., Plance, R.; Soucy, P. Godldstein, S.; Deslongchamps, P. Can. J. Chem. 1993, 71, 695-713.

(9) Michnick, T. J.; Matteson, D. S. Synlett 1991, 631-632.

(10) (a) Smil, David V., Ph. D. Thesis, University of Toronto, p94; (b) Brown, H. C.; Roy, C. D.; Soundararajan Tetrahedron Lett. 1997, 38, 765-768. 
(-)-8-phenylmenthol 9e were purchased from commercial sources and used as received. allylboronate $\mathbf{2 4}$ was prepared according to the procedure of Hoffmann and Schlapbach. ${ }^{11}$ Tetrahydrofuran was distilled from sodium-benzophenone. HMPA and DMPU were distilled over $\mathrm{CaH}_{2}$ before use. Alkyllithiums were titrated according to the Gilman double titration method ${ }^{12}$ prior to use. All other chemicals were purchased from either the Aldrich Chemical Company or Caledon Chemicals and used as received.

Glassware for cuprate reactions was flame-dried under vacuum and then allowed to cool under an inert atmosphere. $\mathrm{NH}_{4} \mathrm{Cl}_{(\text {aq) }}$ and $\mathrm{NaHCO}_{3(\text { aq) }}$ refer to saturated aqueous solutions.

Thin layer chromatography data was performed on Merck Silica Gel $60 \mathrm{~F}_{254}$ plates and were visualized with UV light and either 5\% phosphomolybdic acid in ethanol (PMA) or $1 \% \mathrm{KMnO}_{4(\mathrm{aq})}$. $\mathrm{R}_{f}$ values are approximate. Chiral HPLC analysis was performed using a CHIRALPAK AD-RH column $(0.46 \mathrm{~cm} \times 15 \mathrm{~cm})$ or a CHIRALCEL OD column $(0.46 \mathrm{~cm} \times 25 \mathrm{~cm})$ with UV detection at $210 \mathrm{~nm}$.

NMR spectra were recorded on Bruker AM 300, Bruker AM 200, Varian INOVA-300, INOVA-400 or INOVA-500 MHz instruments. The residual solvent protons $\left({ }^{1} \mathrm{H}\right)$ or the solvent carbons $\left({ }^{13} \mathrm{C}\right)$ were used as internal standards. Boron NMR spectra are referenced to external $\mathrm{BF}_{3} \cdot \mathrm{OEt}_{2}$ and were performed in quartz NMR tubes. ${ }^{1} \mathrm{H}$ NMR data are presented as follows: chemical shift in ppm downfield from tetramethylsilane (multiplicity, coupling constant, integration). The following abbreviations are used in reporting NMR data: s, singlet; br s, broad singlet; d, doublet; t, triplet; q, quartet; qt, quartet of triplets; dd, doublet of doublets; dt, doublet of triplets; $\mathrm{AB}, \mathrm{AB}$ quartet; m, multiplet. High resolution mass spectra were recorded by the University of Alberta Mass Spectrometry Services Laboratory using either electron impact (EI) or electrospray (ES) ionization techniques. Infrared spectra were recorded by University of Alberta Spectral Services and combustion analyses were performed by the University of Alberta Micro-Analytical Lab.

(11) Hoffmann, R. W. ; Schlabach, A. Tetrahedron 1992, 48, 1959-1968.

(12) Taylor, R. K.; Casy, G. In Organocopper Reagents : A Practical Approach; Taylor, R. K., Ed.; Oxford University Press: New York, 1994, pp 53-72. 


\section{X-ray crystallography experimental details}

Experimental. X-ray diffraction data for all compounds were collected with the crystals cooled under a nitrogen stream to $-80{ }^{\circ} \mathrm{C}$. All measurements were carried out using a Bruker PLATFORM diffractometer equipped with a SMART $1000 \mathrm{CCD}$ detector, ${ }^{13}$ and employing Mo $\mathrm{K} \square$ radiation $(\square=0.71073 \AA)$. In all cases data were corrected for absorption (and other systematic effects) through use of a multi-scan method (SADABS). The structures of $\mathbf{2 n}, \mathbf{1 1 f}$, and 27c were solved through use of the direct-methods program SHELXS-86, ${ }^{14}$ while the structure of $\mathbf{2 0}$ was solved using a combined Patterson search/structure expansion method as implemented in the DIRDIF-99 program system. $^{15}$ All structures were refined using full-matrix least-squares methods as implemented in the program SHELXL-93. ${ }^{16}$ Details of the data collections and structure refinements are found in Table S-1; full structure reports will be deposited at the Cambridge Crystallographic Database upon acceptance of this publication.

(13) Programs for diffractometer operation, data collection, data reduction and absorption correction were those supplied by Bruker.

(14) Sheldrick, G. M. Acta Crystallogr. 1990, A46, 467-473.

(15) Beurskens, P. T.; Beurskens, G.; de Gelder, R.; Garcia-Granda, S.; Israel, R.; Gould, R. O.; Smits, J. M. M. (1999). The DIRDIF-99 program system. Crystallography Laboratory, University of Nijmegen, The Netherlands.

(16) Sheldrick, G. M. SHELXL-93. Program for crystal structure determination. University of Göttingen, Germany, 1993. 
Table S-1. Crystallographic Experimental Details

\begin{tabular}{|c|c|c|c|c|}
\hline compound & $2 n$ & 20 & 11f & $27 c$ \\
\hline crystal color & colorless & colorless & colorless & colorless \\
\hline formula & $\mathrm{C}_{18} \mathrm{H}_{15} \mathrm{NO}_{4}$ & $\mathrm{C}_{13} \mathrm{H}_{13} \mathrm{BrO}_{2}$ & $\mathrm{C}_{32} \mathrm{H}_{45} \mathrm{BO}_{4}$ & $\mathrm{C}_{17} \mathrm{H}_{24} \mathrm{O}_{3}$ \\
\hline formula weight & 309.31 & 281.14 & 504.49 & 276.36 \\
\hline crystal dimensions (mm) & $0.34 \square 0.28 \square 0.15$ & $1.01 \square 0.20 \square 0.14$ & $0.66 \square 0.30 \square 0.06$ & $0.52 \square 0.24 \square 0.03$ \\
\hline crystal system & monoclinic & orthorhombic & orthorhombic & orthorhombic \\
\hline space group & $C 2 / c($ No. 15$)$ & $P 2_{1} 2_{1} 2_{1}$ (No. 19$)$ & $P 2_{1}{ }_{2} 2_{1}$ (No. 19$)$ & Pbca (No. 61) \\
\hline \multicolumn{5}{|l|}{ unit cell params $a$} \\
\hline$a(\AA)$ & $13.6632(13)$ & $10.0541(8)$ & $10.9195(14)$ & $12.3717(11)$ \\
\hline$b(\AA)$ & $9.8807(9)$ & $11.0212(9)$ & $14.2580(18)$ & $7.7720(7)$ \\
\hline$c(\AA)$ & $22.901(2)$ & $11.2992(9)$ & $19.034(2)$ & $32.821(3)$ \\
\hline$\square(\mathrm{deg})$ & $98.491(2)$ & & & \\
\hline$V\left(\AA^{3}\right)$ & $3057.8(5)$ & $1252.04(17)$ & $2963.4(7)$ & $3155.8(5)$ \\
\hline$Z$ & 8 & 4 & 4 & 8 \\
\hline$\square_{\text {calcd }}\left(\mathrm{g} \mathrm{cm}^{-3}\right)$ & 1.344 & 1.491 & 1.131 & 1.163 \\
\hline$\mu\left(\mathrm{mm}^{-1}\right)[\square=0.71073 \AA]$ & 0.096 & 3.266 & 0.072 & 0.078 \\
\hline temperature $\left({ }^{\circ} \mathrm{C}\right)$ & -80 & -80 & -80 & -80 \\
\hline total data collected & 8325 & 5302 & 16416 & 17050 \\
\hline ind reflns $\left(R_{\text {int }}\right)$ & $3143(0.0379)$ & $2549(0.0275)$ & $6087(0.0819)$ & $3216(0.0973)$ \\
\hline obsd reflns $\left[F_{\mathrm{O}}^{2} \geq 2 \square\left(F_{\mathrm{O}}^{2}\right)\right]$ & 2330 & 2126 & 3838 & 1975 \\
\hline range of trans factors & $0.9858-0.9682$ & $0.6578-0.1370$ & $0.9957-0.9541$ & $0.9977-0.9605$ \\
\hline data $\left[F_{\mathrm{O}}^{2} \geq-3 \square\left(F_{\mathrm{O}}^{2}\right)\right] /$ params & $3143 / 208$ & $2549 / 145$ & 6087 / 331 & $3216 / 181$ \\
\hline Flack abs structure param $b$ & & $0.018(10)$ & $0.3(15)$ & \\
\hline $\operatorname{GOF}(S)^{c}\left[F_{\mathrm{O}}^{2} \geq-3 \square\left({F_{\mathrm{O}}}^{2}\right)\right]$ & 1.024 & 0.984 & 1.010 & 1.033 \\
\hline \multicolumn{5}{|l|}{ final $R$ indices $d$} \\
\hline$R_{1}\left[F_{\mathrm{O}}^{2} \geq 2 \square\left(F_{\mathrm{O}}^{2}\right)\right]$ & 0.0439 & 0.0321 & 0.0606 & 0.0650 \\
\hline$w R_{2}\left[F_{\mathrm{O}}^{2} \geq-3 \square\left(F_{\mathrm{O}}^{2}\right)\right]$ & 0.1109 & 0.0730 & 0.1284 & 0.1295 \\
\hline largest diff peak, hole (e $\AA^{-3}$ ) & $0.250,-0.164$ & $0.409,-0.377$ & $0.156,-0.170$ & $0.191,-0.173$ \\
\hline
\end{tabular}


$a_{\text {For } 2 n}$, obtained from least-squares refinement of 2947 reflections with $5.11^{\circ}<2 \square<51.98^{\circ} ; 20: 2462$ reflns, $5.16^{\circ}<2 \square<52.25^{\circ}$; 11f: 5822 reflns, $4.69^{\circ}<2 \square<50.67^{\circ} ; 27$ c: 4478 reflns, $5.96^{\circ}<2 \square<51.63^{\circ}$. ${ }^{b}$ Flack, H. D. Acta Crystallogr. 1983, A39, 876-881; Flack, H. D.; Bernardinelli, G. Acta Crystallogr. 1999, A55, 908-915; Flack, H. D.; Bernardinelli, G. J. Appl. Cryst. 2000, 33, 1143-1148. The Flack parameter will refine to a value near zero if the structure is in the correct configuration, or to a value near one for the inverted configuration. For 11f, although the Flack parameter has refined to a value indicative of a degree of racemic twinning, the large value of the standard error for this parameter and the presence of only light atoms (oxygen, carbon, boron and hydrogen) in the molecule suggest that absolute configuration cannot be assigned based on X-ray data alone; the assignment of absolute configuration of 11f is based upon the known stereochemistry of the precursor compound. $c S=\left[\square w\left(F_{\mathrm{o}}^{2}-F_{\mathrm{c}}^{2}\right)^{2} /(n-p)\right]^{1 / 2}(n=$ number of data; $p=$ number of parameters varied; $w=\left[\square^{2}\left(F_{\mathrm{o}}^{2}\right)+\left(A_{0} P\right)^{2}+A_{1} P\right]^{-1}$ where $P=\left[\operatorname{Max}\left(F_{\mathrm{o}}^{2}, 0\right)+2 F_{\mathrm{c}}^{2}\right] / 3$ and the values of $A_{0}$ and $A_{1}$ are optimized during refinement; for 2n, $A_{0}=0.0496, A_{1}=1.2237$; for 2o, $A_{0}=0, A_{1}=0$; for 11f, $A_{0}=0.0469, A_{1}=0.1044$; for 27c, $A_{0}=0.0381, A_{1}=1.6070$ ). $d R_{1}=\square|| F_{\mathrm{o}}|-| F_{\mathrm{c}} \| / \square\left|F_{\mathrm{o}}\right| ; w R_{2}=\left[\square w\left(F_{\mathrm{o}}^{2}-F_{\mathrm{c}}^{2}\right)^{2} / \square w\left(F_{\mathrm{o}}{ }^{4}\right)\right]^{1 / 2}$. 


\section{Stereochemical Assignments}

\section{1 nOe enhancements for boronate $1 \mathrm{c}\left(\mathrm{CDCl}_{3}, 300 \mathrm{MHz}\right)$}

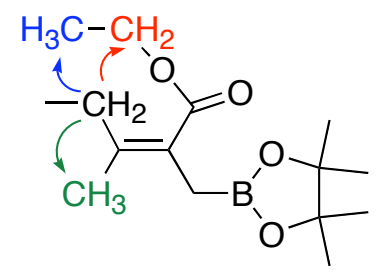

Irradiation at the $\mathrm{Z}-\mathrm{CH}_{2}$ group causes enhancement of the signals for the two groups of protons on the ethyl ester but not of the signal for the $\mathrm{CH}_{2}-\mathrm{B}$ protons. Unfortunately, the chemical shift of the $E$-methyl group ( $\square 1.73 \mathrm{ppm}$ ) is too close to that of the methylene adjacent to the boron ( $\square 1.82 \mathrm{ppm}$ ) to allow for selective irradiation.

3.2 nOe enhancements for lactones $2 \mathrm{i}$ and $2 \mathrm{j}\left(\mathrm{CDCl}_{3}, 300 \mathrm{MHz}\right)$
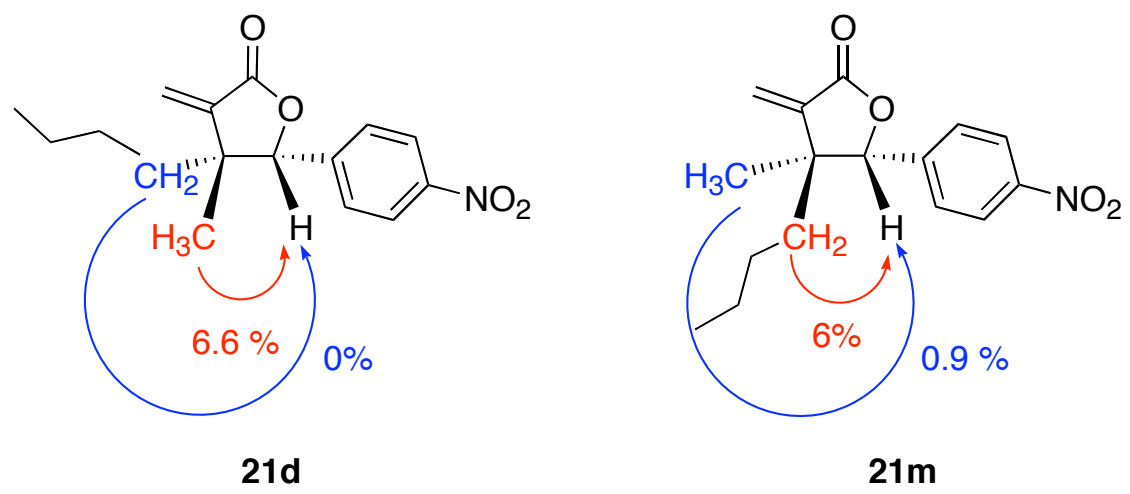
3.3 X-ray crystal structure of lactone $2 n$ (ORTEP, with Chemdraw structure for reference)
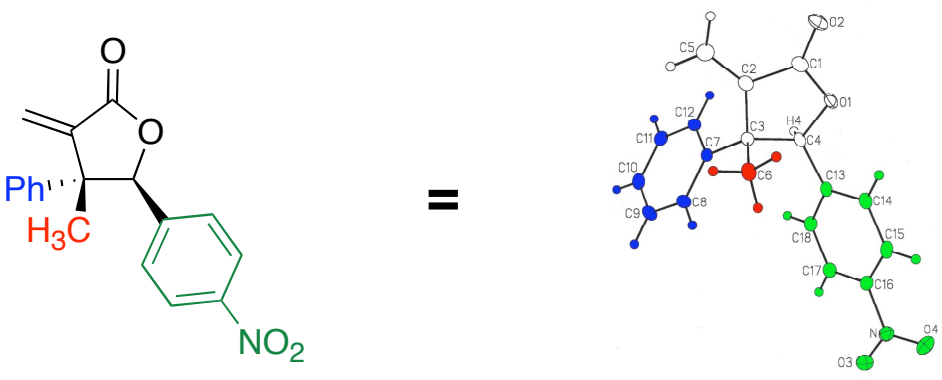

2n

3.4 X-ray crystal structure of lactone 2o (ORTEP, with Chemdraw structure for reference)
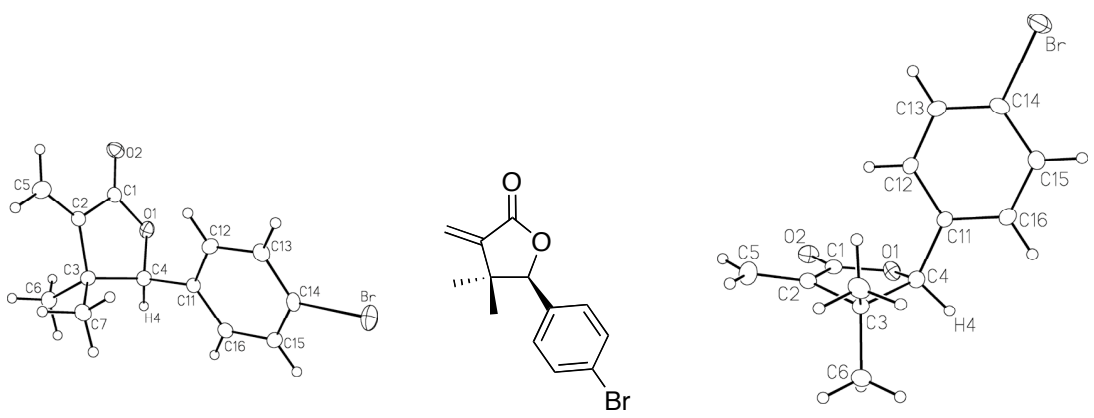

20

3.5 X-ray crystal structure of $\square$-methyl lactone 27c (ORTEPs, with Chemdraw structure for reference)
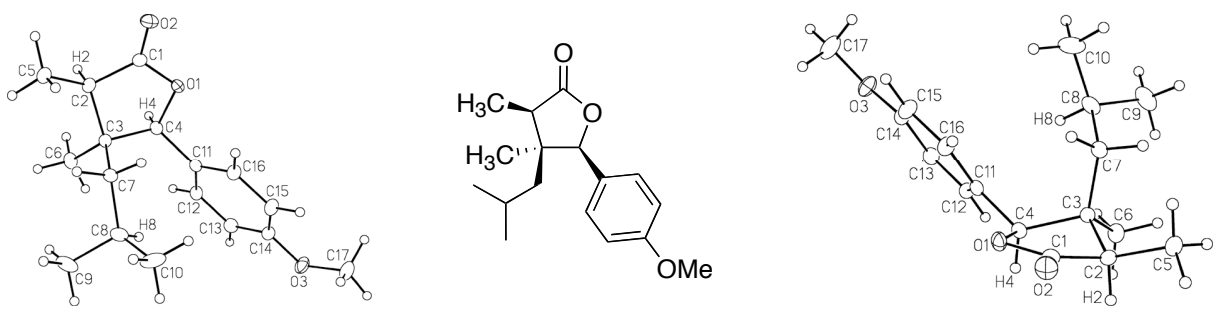

27c 


\section{Preparation of 2-carboxyester 3,3-disubstituted allylboronates 1}

\subsection{Allylboronates 1 from 3 equivalents of electrophile $5 a$}

\subsubsection{Ethyl 2-[(4,4,5,5-tetramethyl-1,3,2-dioxaborolan-2-yl)methyl]-3-methylbut-2-enoate 1b}

A slurry of $\mathrm{CuBr} \cdot \mathrm{SMe}_{2}(1.35 \mathrm{~g}, 6.58 \mathrm{mmol})$ in THF $(15 \mathrm{~mL})$ at $0{ }^{\circ} \mathrm{C}$ under Ar was treated with MeLi (1.18 M in ether, $11.2 \mathrm{~mL}, 13.2 \mathrm{mmol})$. When the clear, colourless solution had formed ( $\sim 10 \mathrm{~min})$ the flask was placed in an acetone $/ \mathrm{CO}_{2}$ bath and treated via canula with a $-78{ }^{\circ} \mathrm{C}$ solution of ethyl 2-butynoate $(0.721 \mathrm{~g}, 6.43 \mathrm{mmol})$ in THF $(2.5 \mathrm{~mL}+$ $2.5 \mathrm{~mL}$ canula rinse). The resulting light brown mixture was stirred at $-78{ }^{\circ} \mathrm{C}$ for $1 \mathrm{~h}$ and then injected sequentially with iodomethaneboronate $5 \mathbf{a}(4.85 \mathrm{~g}, 18.10 \mathrm{mmol})$ and DMPU $(15 \mathrm{~mL})$. After 10 minutes the mixture was brought to $0{ }^{\circ} \mathrm{C}$, left to stir for $2 \mathrm{~h}$, and then the reaction was quenched with $\mathrm{NH}_{4} \mathrm{Cl}_{(\mathrm{aq})}(25 \mathrm{~mL})$. The layers were separated and the aqueous layer was extracted with ether $(4 \times 25 \mathrm{~mL})$. The combined organic layers were washed with water $(6 \times 25 \mathrm{~mL})$ and brine $(25 \mathrm{~mL})$, dried $\left(\mathrm{Na}_{2} \mathrm{SO}_{4}\right)$, and concentrated to give the product as a yellow oil (1.554 $\mathrm{g}, 90 \%)$.

TLC (25\% Et $\left.\mathrm{E}_{2} \mathrm{O} / \mathrm{Hexane}_{\mathrm{KMnO}}\right): 0.36 ;{ }^{1} \mathrm{H}$ NMR (300 $\left.\mathrm{MHz}, \mathrm{CDCl}_{3}\right): \square 4.14$ (q, $J=7.1 \mathrm{~Hz}, 2 \mathrm{H}), 2.06(\mathrm{~s}, 3 \mathrm{H}), 1.86(\mathrm{~s}, 2 \mathrm{H}), 1.77(\mathrm{~s}, 3 \mathrm{H}), 1.26(\mathrm{t}, J=7.1 \mathrm{~Hz}, 3 \mathrm{H}), 1.22$ (s, $12 \mathrm{H}) ;{ }^{13} \mathrm{C} \mathrm{NMR}\left(50 \mathrm{MHz}, \mathrm{CDCl}_{3}\right)$ : $\square$ 169.4, 142.4, 123.7, 83.1, 59.9, 24.7, 23.0, 14.3; ${ }^{11} \mathrm{~B}$ NMR (64 MHz, $\left.\mathrm{CDCl}_{3}\right)$ : $\square$ 32.7; IR $\left(\mathrm{CH}_{2} \mathrm{Cl}_{2}\right.$ cast, $\left.\mathrm{cm}^{-1}\right)$ : 2979, 1713, 1635, 1148; HRMS (EI, $m / z$ ) Calcd for $\mathrm{C}_{14} \mathrm{H}_{25}{ }^{11} \mathrm{BO}_{4}$ : 268.18460. Found: 268.18450.

\subsubsection{Ethyl (2Z)-2-[(4,4,5,5-tetramethyl-1,3,2-dioxaborolan-2-yl)methyl]-3-methylpent-2-enoate 1c}

A slurry of $\mathrm{CuBr} \cdot \mathrm{SMe}_{2}(1.11 \mathrm{~g}, 5.39 \mathrm{mmol})$ in $\mathrm{THF}(7.5 \mathrm{~mL})$ at $0{ }^{\circ} \mathrm{C}$ under Ar was treated with $\mathrm{MeLi}\left(1.27 \mathrm{M}\right.$ in $\left.\mathrm{Et}_{2} \mathrm{O}, 8.6 \mathrm{~mL}, 11 \mathrm{mmol}\right)$. When the clear, colourless solution had formed ( $10 \mathrm{~min})$ the flask was placed in an acetone/ $\mathrm{CO}_{2}$ bath and treated via canula with a $-78{ }^{\circ} \mathrm{C}$ solution of ethyl 2-pentynoate $(674 \mathrm{mg}, 5.34 \mathrm{mmol})$ in THF $(2.5 \mathrm{~mL}+$ $2.5 \mathrm{~mL}$ canula rinse). The resulting light brown mixture was stirred at $-78{ }^{\circ} \mathrm{C}$ for $1 \mathrm{~h}$ and then injected sequentially with iodomethaneboronate 5a $(3.700 \mathrm{mg}, 13.81 \mathrm{mmol})$ and HMPA $(7.5 \mathrm{~mL})$. After 10 minutes the mixture was brought to $0{ }^{\circ} \mathrm{C}$, left to stir for $2 \mathrm{~h}$, and then reaction was quenched with $\mathrm{NH}_{4} \mathrm{Cl}_{(\mathrm{aq})}(25 \mathrm{~mL})$. The layers were separated and the aqueous layer was extracted with ether $(4 \times 25 \mathrm{~mL})$. The combined organic layers were washed with water $(6 \times 25 \mathrm{~mL})$ and brine $(25 \mathrm{~mL})$, dried $\left(\mathrm{Na}_{2} \mathrm{SO}_{4}\right)$, and concentrated to give 
the product as a yellow oil $(1.090 \mathrm{~g}, 3.834 \mathrm{mmol}, 72 \%)$. The product was purified by Kugelrohr distillation $\left(0.1\right.$ torr, $\left.200{ }^{\circ} \mathrm{C}\right)$ to give a colourless oil $(1.020 \mathrm{~g}, 3.614 \mathrm{mmol}, 68 \%)$. TLC (25\% Et $\left.2 \mathrm{O} / \mathrm{Hexane}, \mathrm{KMnO}_{4}\right): 0.35 ;{ }^{1} \mathrm{H} \mathrm{NMR}\left(300 \mathrm{MHz}, \mathrm{CDCl}_{3}\right): 04.13$ (q, $J=7.1 \mathrm{~Hz}, 2 \mathrm{H}), 2.36$ (q, $J=7.4,2 \mathrm{H}), 1.82(\mathrm{~s}, 2 \mathrm{H}), 1.75(\mathrm{~s}, 3 \mathrm{H}), 1.26(\mathrm{t}, J=7.1,3 \mathrm{H}), 1.21$ (s, 12H), 1.02 (t, $J=7.4,3 \mathrm{H}) ;{ }^{13} \mathrm{C}$ NMR (50 MHz, $\mathrm{CDCl}_{3}$ ): $\square$ 169.2, 147.1, 123.3, 83.0, 59.8, 29.5, 24.6, 20.2, 14.2, 12.8; ${ }^{11} \mathrm{~B}$ NMR (64 MHz, $\mathrm{CDCl}_{3}$ ): $\square$ 32.7, 22.3 (impurity); IR $\left(\mathrm{CH}_{2} \mathrm{Cl}_{2}\right.$ cast, $\left.\mathrm{cm}^{-1}\right): 2978,1713,1633,1147$; HRMS (EI, $\left.m / z\right)$ Calcd for $\mathrm{C}_{15} \mathrm{H}_{27}{ }^{11} \mathrm{BO}_{4}$ : 282.20023. Found: 282.20020 .

\subsubsection{Methyl (2Z)-2-[(4,4,5,5-tetramethyl-1,3,2-dioxaborolan-2-yl)methyl]-3-methylhept-2- enoate 1d}

This compound was prepared according to the procedure in Section 4.1.2, with $\mathrm{CuBr} \cdot \mathrm{SMe}_{2}$ (225 mg, $\left.1.01 \mathrm{mmol}\right)$, MeLi (1.47 M in ether, $\left.1.5 \mathrm{~mL}, 2.2 \mathrm{mmol}\right)$, methyl 2-heptynoate 3d (156 mg, $1.12 \mathrm{mmol})$ and iodomethaneboronate $5 \mathbf{a}(0.5 \mathrm{~mL}, 3 \mathrm{mmol})$ using DMPU $(5 \mathrm{~mL})$ instead of HMPA to give the desired allylboronate as a yellow oil (251 mg, 85\%).

$\operatorname{TLC}\left(25 \% \mathrm{Et}_{2} \mathrm{O} / \mathrm{Hexane}, \mathrm{KMnO}_{4}\right): 0.40 ;{ }^{1} \mathrm{H} \mathrm{NMR}\left(400 \mathrm{MHz}, \mathrm{CDCl}_{3}\right): \square 3.68(\mathrm{~s}, 3 \mathrm{H})$, $2.41(\mathrm{~d}, J=8 \mathrm{~Hz}, 2 \mathrm{H}), 1.84(\mathrm{~s}, 2 \mathrm{H}), 1.79(\mathrm{~s}, 3 \mathrm{H}), 1.41(\mathrm{~m}, 2 \mathrm{H}), 1.32(\mathrm{~m}, 2 \mathrm{H}), 1.25(\mathrm{~m}, 12 \mathrm{H}$ + impurities), 0.90 (t, $J=4 \mathrm{~Hz}, 3 \mathrm{H}) ;{ }^{13} \mathrm{C}$ NMR (50 MHz, $\mathrm{CDCl}_{3}$ ): $\square$ 169.9, 146.9, 123.4, 83.1, 51.0, 36.1, 30.6, 24.6, 22.6, 20.9, 15.0, 14.0; ${ }^{11} \mathrm{~B}$ NMR (128 MHz, $\left.\mathrm{CDCl}_{3}\right)$ : $\square$ 34.0, 22.3 (impurity); IR $\left(\mathrm{CH}_{2} \mathrm{Cl}_{2}\right.$ cast, $\left.\mathrm{cm}^{-1}\right)$ : 2978, 1718, 1629, 1109; HRMS (EI, $m / z$ ) Calcd for $\mathrm{C}_{16} \mathrm{H}_{29}{ }^{11} \mathrm{BO}_{4}$ : 296.21588. Found: 296.21643.

\subsubsection{Ethyl (2E)-2-[(4,4,5,5-tetramethyl-1,3,2-dioxaborolan-2-yl)methyl]-3-methylhept-2-enoate 10}

The dibutylcuprate was formed by adding $n$-BuLi $(1.6 \mathrm{M}$ in Hexanes, $1.3 \mathrm{~mL}$, $2.1 \mathrm{mmol})$ to a slurry of $\mathrm{CuBr} \cdot \mathrm{SMe}_{2}(207 \mathrm{mg}, 1.00 \mathrm{mmol})$ in $\mathrm{THF}(3 \mathrm{~mL})$ at $-40{ }^{\circ} \mathrm{C}$. After the dark brown homogenous solution formed ( $\sim 30 \mathrm{~min})$, the preparation was carried out as described in Section 4.1.2, with ethyl 2-butynoate $(130 \mathrm{mg}, 1.16 \mathrm{mmol}$ ) and iodomethaneboronate $\mathbf{5 a}(807 \mathrm{mg}, 3.01 \mathrm{mmol})$. The desired allylboronate was obtained as a yellow oil (466 mg, >100\%).

${ }^{1} \mathrm{H}$ NMR (300 MHz, $\mathrm{CDCl}_{3}$ ): $\square 4.18$ (q, $\left.J=6 \mathrm{~Hz}, 2 \mathrm{H}\right), 2.09$ (m, 2H), 2.01 (s, 3H), 1.86 (s, $2 \mathrm{H}), 1.38(\mathrm{~m}, 2 \mathrm{H}), 1.24(\mathrm{~m}, 12 \mathrm{H}+$ impurities $), 0.89(\mathrm{~m}, 3 \mathrm{H}+$ hydrocarbon impurities);

${ }^{13} \mathrm{C} \mathrm{NMR}\left(75 \mathrm{MHz}, \mathrm{CDCl}_{3}\right)$ : $\square 169.5,146.4,123.5,83.1,59.9,36.8,29.6,24.6,22.9,21.2$, 
14.3, 14.0; ${ }^{11} \mathrm{~B}$ NMR (64 MHz, $\left.\mathrm{CDCl}_{3}\right)$ : $\square 32.3,22.0$ (impurity); IR $\left(\mathrm{CH}_{2} \mathrm{Cl}_{2}\right.$ cast, $\left.\mathrm{cm}^{-1}\right)$ : 2978, 1714, 1627, 1148; HRMS (EI, $m / z$ ) Calcd for $\mathrm{C}_{17} \mathrm{H}_{31}{ }^{11} \mathrm{BO}_{4}$ : 310.23154. Found: 310.23185 .

\subsubsection{Ethyl (2E)-2-[(4,4,5,5-tetramethyl-1,3,2-dioxaborolan-2-yl)methyl]-3,5-dimethylhex-2- enoate $1 f$}

The dibutylcuprate was formed by adding isobutylmagnesium chloride $(2.0 \mathrm{M}$ in THF, $1.1 \mathrm{~mL}, 2.2 \mathrm{mmol})$ to a slurry of $\mathrm{CuBr} \cdot \mathrm{SMe}_{2}(222 \mathrm{mg}, 1.08 \mathrm{mmol})$ in THF $(1.5 \mathrm{~mL})$ at $-40{ }^{\circ} \mathrm{C}$. After a black mixture formed $(\sim 30 \mathrm{~min})$, the preparation was carried out described in Section 4.1.2, with ethyl 2-butynoate $(121 \mathrm{mg}, 1.08 \mathrm{mmol})$ and iodomethaneboronate $5 \mathbf{a}(0.96 \mathrm{~g}, 3.6 \mathrm{mmol})$. The product was then purified by flash chromatography (25\% $\mathrm{Et}_{2} \mathrm{O} / \mathrm{Hexanes}, 23 \mathrm{~g}$ silica) to give the desired allylboronate (149 $\mathrm{mg}$, $0.481 \mathrm{mmol}, 44 \%)$.

TLC (25\% Et 2 O/Hexane, $\left.\mathrm{KMnO}_{4}\right): 0.48 ;{ }^{1} \mathrm{H}$ NMR (400 $\left.\mathrm{MHz}, \mathrm{CDCl}_{3}\right): \square 4.15$ (q, $J=7.1 \mathrm{~Hz}, 2 \mathrm{H}), 2.02(\mathrm{~d}, J=7.3 \mathrm{~Hz}, 2 \mathrm{H}), 2.00(\mathrm{~s}, 3 \mathrm{H}), 1.90(\mathrm{br} \mathrm{s}, 2 \mathrm{H}) 1.86(\mathrm{~m}, 1 \mathrm{H}), 1.27(\mathrm{t}$, $J=7.1 \mathrm{~Hz}, 3 \mathrm{H}), 1.21(\mathrm{~s}, 12 \mathrm{H}), 0.89(\mathrm{~d}, J=6.6 \mathrm{~Hz}, 6 \mathrm{H}) ;{ }^{13} \mathrm{C}$ NMR $\left(75 \mathrm{MHz}, \mathrm{CDCl}_{3}\right)$ : प169.5, 145.2, 124.7, 83.1, 59.9, 45.6, 27.2, 24.7, 22.6, 21.7; ${ }^{11} \mathrm{~B}$ NMR (64 MHz, $\left.\mathrm{CDCl}_{3}\right)$ : प32.6; IR $\left(\mathrm{CH}_{2} \mathrm{Cl}_{2}\right.$ cast, $\left.\mathrm{cm}^{-1}\right): 2977,1715,1624,1349,1148$; HRMS (EI, $\left.m / z\right)$ Calcd for $\mathrm{C}_{17} \mathrm{H}_{31}{ }^{11} \mathrm{BO}_{4}$ : 310.23154. Found: 310.23236.

\subsubsection{Ethyl (2E)-2-[(4,4,5,5-tetramethyl-1,3,2-dioxaborolan-2-yl)methyl]-3,4-dimethylhex-2- enoate $1 \mathrm{~g}$}

The dibutylcuprate was formed by adding $(s)$-BuLi (1.40 M in cyclohexane, $1.6 \mathrm{~mL}$, $2.2 \mathrm{mmol})$ to a slurry of $\mathrm{CuBr} \cdot \mathrm{SMe}_{2}(226 \mathrm{mg}, 1.10 \mathrm{mmol})$ in $\mathrm{THF}(1 \mathrm{~mL})$ at $-40{ }^{\circ} \mathrm{C}$. After a black mixture formed ( $\sim 30 \mathrm{~min})$, the preparation was carried out as described in Section 4.1.2, with ethyl 2-butynoate $(117 \mathrm{mg}, 1.04 \mathrm{mmol})$ and iodomethaneboronate $5 \mathbf{5 a}(1.06 \mathrm{~g}$, $3.94 \mathrm{mmol})$. The product was then purified by Kugelrohr distillation $\left(175^{\circ} \mathrm{C}, 0.1\right.$ torr) to give the desired allylboronate ( $219 \mathrm{mg}, 0.706 \mathrm{mmol}, 70 \%$ ).

TLC (25\% Et 2 O/Hexane, $\left.\mathrm{KMnO}_{4}\right): 0.50 ;{ }^{1} \mathrm{H}$ NMR (500 MHz, $\left.\mathrm{CDCl}_{3}\right): 04.14$ (q, $J=7.0 \mathrm{~Hz}, 2 \mathrm{H}), 2.54(\mathrm{~m}, 1 \mathrm{H}), 1.90(\mathrm{~d}, J=3.4 \mathrm{~Hz}, 2 \mathrm{H}), 1.82(\mathrm{t}, J=1 \mathrm{~Hz}, 3 \mathrm{H}), 1.36(\mathrm{~m}, 2 \mathrm{H})$, $1.26(\mathrm{t}, J=7.2 \mathrm{~Hz}, 3 \mathrm{H}), 1.20(\mathrm{~s}, 12 \mathrm{H}), 0.96(\mathrm{~d}, J=6.7 \mathrm{~Hz}, 3 \mathrm{H}), 0.81(\mathrm{t}, J=7.5 \mathrm{~Hz}, 3 \mathrm{H})$;

${ }^{13} \mathrm{C} \mathrm{NMR}\left(125 \mathrm{MHz}, \mathrm{CDCl}_{3}\right)$ : $\square 169.7,148.0,123.5,83.0,59.8,38.7,27.4,24.8,24.7,18.0$, 
14.5, 14.3, 12.2; ${ }^{11} \mathrm{~B}$ NMR (64 MHz, $\left.\mathrm{CDCl}_{3}\right)$ : 732.7 ; IR $\left(\mathrm{CH}_{2} \mathrm{Cl}_{2}\right.$ cast, $\left.\mathrm{cm}^{-1}\right): 2976,1713$, 1616, 1146; HRMS (EI, $m / z$ ) Calcd for $\mathrm{C}_{17} \mathrm{H}_{31}{ }^{11} \mathrm{BO}_{4}$ : 310.23154. Found: 310.23180 .

\subsubsection{Ethyl (2E)-2-[(4,4,5,5-tetramethyl-1,3,2-dioxaborolan-2-yl)methyl]-3,4,4-trimethylpent-2- enoate $1 \mathrm{~h}$}

The dibutylcuprate was formed by adding $(t)$ - $\mathrm{BuLi}(1.54 \mathrm{M}$ in pentane, $1.3 \mathrm{~mL}$, $2.0 \mathrm{mmol})$ to a slurry of $\mathrm{CuBr} \cdot \mathrm{SMe}_{2}(203 \mathrm{mg}, 0.989 \mathrm{mmol})$ in THF $(1 \mathrm{~mL})$ at $-40{ }^{\circ} \mathrm{C}$. After a black mixture formed ( $60 \mathrm{~min})$, the preparation was carried out as described in Section 4.1.2, with ethyl 2-butynoate $(110 \mathrm{mg}, 0.986 \mathrm{mmol})$ and iodomethaneboronate $\mathbf{5 a}$ $(911 \mathrm{~g}, 3.41 \mathrm{mmol})$. The product was then purified by silica gel chromatography (25\% $\mathrm{Et}_{2} \mathrm{O} / \mathrm{Hexane}$ ) to give the desired allylboronate as a colourless oil (222 $\mathrm{mg}$, $0.714 \mathrm{mmol}, 72 \%)$ as a 2:1 mixture of diastereomers.

TLC (25\% Et 2 O/Hexane, $\left.\mathrm{KMnO}_{4}\right): 0.38 ;{ }^{1} \mathrm{H}$ NMR $\left(500 \mathrm{MHz}, \mathrm{CDCl}_{3}\right)$ : Major Isomer: $\square 4.16$ (q, $J=7.2 \mathrm{~Hz}, 2 \mathrm{H}$ ), 2.06 (br s, 2H), 1.79 (s, 3H), 1.27 (t, $J=7.2 \mathrm{~Hz}, 3 \mathrm{H}$ ), 1.21 (s, 12H), 1.17 (s, 9H); Minor Isomer: $\square 4.12$ (q, $J=7.2 \mathrm{~Hz}, 2 \mathrm{H}), 1.77$ (br s, 2H), $1.65(\mathrm{~s}, 3 \mathrm{H})$, $1.27(\mathrm{t}, J=7.2 \mathrm{~Hz}, 3 \mathrm{H}), 1.21(\mathrm{~s}, 12 \mathrm{H}), 1.09(\mathrm{~s}, 9 \mathrm{H}) ;{ }^{13} \mathrm{C} \mathrm{NMR}^{17}\left(125 \mathrm{MHz}, \mathrm{CDCl}_{3}\right)$ : प173.3, 172.2, 145.1, 140.6, 126.1, 122.8, 83.2, 83.1, 60.1, 37.3, 36.3, 30.0, 29.4, 24.8, 24.7, 19.8, 16.6, 14.0; ${ }^{11} \mathrm{~B}$ NMR (64 MHz, $\left.\mathrm{CDCl}_{3}\right)$ : 732.1 ; IR $\left(\mathrm{CH}_{2} \mathrm{Cl}_{2}\right.$ cast, $\left.\mathrm{cm}^{-1}\right): 2978,1720$, 1610, 1341, 1143; HRMS (EI, $m / z$ ) Calcd for $\mathrm{C}_{17} \mathrm{H}_{31}{ }^{11} \mathrm{BO}_{4}$ : 310.23154. Found: 310.23241 .

\subsubsection{Methyl (2E)-2-[(4,4,5,5-tetramethyl-1,3,2-dioxaborolan-2-yl)methyl]-3-butylundec-2- enoate $1 \mathbf{j}$}

The dioctylcuprate was formed by adding octylmagnesium chloride (2.0 M in THF, $1.1 \mathrm{~mL}, 2.2 \mathrm{mmol})$ to a slurry of $\mathrm{CuBr} \cdot \mathrm{SMe}_{2}(228 \mathrm{mg}, 1.11 \mathrm{mmol})$ in $\mathrm{THF}(3.0 \mathrm{~mL})$ at $0{ }^{\circ} \mathrm{C}$. After a purple mixture formed $(\sim 2 \mathrm{~min})$, flask was immersed in a $-78{ }^{\circ} \mathrm{C}$ bath and the preparation was carried out as described in Section 4.1.2, with methyl 2-heptynoate 3d $(154 \mathrm{mg}, 1.10 \mathrm{mmol})$ and iodomethaneboronate $5 \mathbf{a}(0.97 \mathrm{~g}, 3.6 \mathrm{mmol})$. The product was then purified by flash chromatography $\left(10 \% \mathrm{Et}_{2} \mathrm{O} / \mathrm{Hexanes}, 22 \mathrm{~g}\right.$ silica) to give the desired allylboronate that show some hydrocarbon impurities (104 mg, $0.264 \mathrm{mmol}, 24 \%$ ).

TLC (10\% Et 2 O/Hexane, $\mathrm{KMnO}_{4}$ ): 0.29; ${ }^{1} \mathrm{H}$ NMR (300 MHz, $\mathrm{CDCl}_{3}$ ): 73.65 (s, 3H), 2.35 (m, 2H), $2.06(\mathrm{~m}, 2 \mathrm{H}), 1.81$ (s, 2H), 1.44-1.18 (m, $16 \mathrm{H}), 1.20(\mathrm{~s}, 12 \mathrm{H}), 0.87$ (m,6H); 
${ }^{13} \mathrm{C}$ NMR (125 MHz, $\mathrm{CDCl}_{3}$ ): $\square 169.4,151.4,123.2,83.0,51.0,34.7,34.3,31.9,31.2,30.1$, 29.6, 29.3, 27.9, 24.7, 23.0, 22.7, 14.1, 14.0; ${ }^{11} \mathrm{~B} \mathrm{NMR}\left(128 \mathrm{MHz}, \mathrm{CDCl}_{3}\right)$ : $\square$ 32.7; IR $\left(\mathrm{CH}_{2} \mathrm{Cl}_{2}\right.$ cast, $\left.\mathrm{cm}^{-1}\right): 2927,1720,1623,1348,1147$; HRMS (EI, $\left.\mathrm{m} / z\right)$ Calcd for $\mathrm{C}_{23} \mathrm{H}_{43}{ }^{11} \mathrm{BO}_{4}$ : 394.32544 . Found: 394.32545 .

\subsubsection{Methyl (2E)-2-[(4,4,5,5-tetramethyl-1,3,2-dioxaborolan-2-yl)methyl]-3-methylhexa-2,5- dienoate $1 \mathrm{k}$}

The cuprate was formed by adding allylmagnesium chloride (2.0 M in THF, $1.0 \mathrm{~mL}$, $2.0 \mathrm{mmol})$ to a slurry of $\mathrm{CuBr} \cdot \mathrm{SMe}_{2}(209 \mathrm{mg}, 1.02 \mathrm{mmol})$ in $\mathrm{THF}(3 \mathrm{~mL})$ at $-40{ }^{\circ} \mathrm{C}$. After a tan mixture formed ( $\sim 30 \mathrm{~min})$, the preparation was carried out as described in Section 4.1.2, with methyl 2-butynoate $(99 \mathrm{mg}, 1.0 \mathrm{mmol})$ and iodomethaneboronate $\mathbf{5 a}(0.83 \mathrm{~g}$, $3.1 \mathrm{mmol})$. The product was then purified by flash chromatography $\left(25 \% \mathrm{Et}_{2} \mathrm{O} / \mathrm{Hexanes}\right.$, $30 \mathrm{~g}$ silica) to give the desired allylboronate $(170 \mathrm{mg}, 0.606 \mathrm{mmol}, 60 \%)$.

$\operatorname{TLC}\left(25 \% \mathrm{Et}_{2} \mathrm{O} / \mathrm{Hexane}, \mathrm{KMnO}_{4}\right): 0.48 ;{ }^{1} \mathrm{H}$ NMR $\left(500 \mathrm{MHz}, \mathrm{CDCl}_{3}\right)$ : $5.74(\mathrm{~m}, 1 \mathrm{H})$, $5.03(\mathrm{~m}, 2 \mathrm{H}), 3.68(\mathrm{~s}, 3 \mathrm{H}), 2.86(\mathrm{~d}, 2 \mathrm{H}), 2.01$ (s, 3H), 1.87 (br s, 2H), $1.20(\mathrm{~s}, 12 \mathrm{H})$;

${ }^{13} \mathrm{C}$ NMR (125 MHz, $\mathrm{CDCl}_{3}$ ): $\square$ 169.3, 143.5, 133.9, 124.6, 116.1, 83.1, 51.1, 40.9, 24.7,

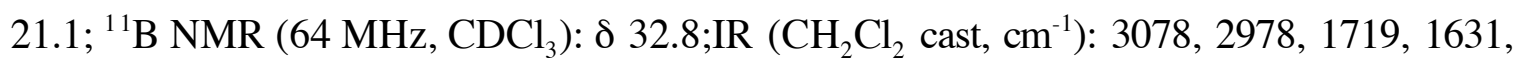
1349, 1146, 993, 914; HRMS (EI, $m / z$ ) Calcd for $\mathrm{C}_{15} \mathrm{H}_{25}{ }^{11} \mathrm{BO}_{4}$ : 280.18460. Found: 280.18496 .

\subsubsection{Methyl (2Z)-4-(tert-butyldiphenylsilyloxy)-2-[(4,4,5,5-tetramethyl-1,3,2-dioxaborolan-2- yl)methyl]-3-methylbut-2-enoate 1 I}

This compound was prepared according to the procedure in Section 4.1.2, with $\mathrm{CuBr} \cdot \mathrm{SMe}_{2}$ (210 mg, $\left.1.02 \mathrm{mmol}\right)$, MeLi (1.27 M in ether, $\left.1.6 \mathrm{~mL}, 2.0 \mathrm{mmol}\right)$, methyl 4-(tert-butyldiphenylsilyloxy)-2-butynoate $3 \mathbf{e}(368 \mathrm{mg}, \quad 1.01 \mathrm{mmol})$ and iodomethaneboronate $5 \mathbf{a}(846 \mathrm{mg}, 3.16 \mathrm{mmol})$ to give the crude allylboronate as an oil $(556 \mathrm{mg})$. Purification by flash chromatography $\left(25 \% \mathrm{Et}_{2} \mathrm{O} / \mathrm{Hexane}, 27 \mathrm{~g}\right.$ silica) gave the desired allylboronate (308 $\mathrm{mg}, 0.606 \mathrm{mmol}, 60 \%)$.

TLC (25\% Et 2 O/Hexane, $\mathrm{KMnO}_{4}$ ): 0.41; ${ }^{1} \mathrm{H}$ NMR (500 MHz, CDCl $)$ : $7.64(\mathrm{~m}, 4 \mathrm{H})$, 7.37 (m, 6H), 4.58 (s, 2H), 3.47 (s, 3H), 1.94 (s, 3H), 1.86 (s, 2H), 1.21 (s, 12H), 1.03 (s, $3 \mathrm{H}) ;{ }^{13} \mathrm{C}$ NMR (125 MHz, $\mathrm{CDCl}_{3}$ ): $\square$ 168.6, 145.6, 135.5, 133.7, 129.4, 127.7, 127.5, 124.3, 83.2, 65.2, 51.2, 27.0, 24.9, 19.5, 17.2; ${ }^{11} \mathrm{~B}$ NMR (64 $\mathrm{MHz}, \mathrm{CDCl}_{3}$ ): 732.2 ; 
IR $\left(\mathrm{CH}_{2} \mathrm{Cl}_{2}\right.$ cast, $\left.\mathrm{cm}^{-1}\right): 3071,2856,1715,1634,1589,1347,1112,1070,741,702$; HRMS (ES, $m / z$ ) Calcd for $\mathrm{C}_{29} \mathrm{H}_{41}{ }^{11} \mathrm{BSiO}_{5} \mathrm{Na}$ : 531.271403. Found: 531.270743.

\subsubsection{Methyl (2Z)-5-(tert-butyldiphenylsilyloxy)-2-[(4,4,5,5-tetramethyl-1,3,2-dioxaborolan-2- yl)methyl]-3-methylpent-2-enoate $1 \mathrm{~m}$}

This compound was prepared according to the procedure in Section 4.1.2, with $\mathrm{CuBr} \cdot \mathrm{SMe}_{2}(221 \mathrm{mg}, 1.07 \mathrm{mmol}), \mathrm{MeLi}(1.27 \mathrm{M}$ in ether, $1.7 \mathrm{~mL}, 2.1 \mathrm{mmol})$, methyl 5-(tert-butyldiphenylsilyloxy)-2-pentynoate $3 f(404 \mathrm{mg}, 1.06 \mathrm{mmol})$ and iodomethaneboronate $\mathbf{5 a}(752 \mathrm{mg}, 2.81 \mathrm{mmol})$ to give the crude allylboronate as an oil (487 mg, 92\%).

${ }^{1} \mathrm{H}$ NMR (500 MHz, $\left.\mathrm{CDCl}_{3}\right): \square 7.64(\mathrm{~m}, 4 \mathrm{H}), 7.36(\mathrm{~m}, 6 \mathrm{H}), 3.77(\mathrm{~d}, J=6.3 \mathrm{~Hz}, 2 \mathrm{H})$, $3.61(\mathrm{~s}, 3 \mathrm{H}), 2.73(\mathrm{~d}, J=6.3 \mathrm{~Hz}, 2 \mathrm{H}), 1.84$ (s, 2H), 1.76 (s, 3H), 1.19 (s, 12H), 1.01 (s, 9H); ${ }^{13} \mathrm{C}$ NMR (125 MHz, $\mathrm{CDCl}_{3}$ ): $\square 169.0,143.9,135.5,133.9,129.4,127.5,125.2,83.1,63.3$, 51.1, 39.5, 27.0, 24.9, 24.8, 19.3; ${ }^{1} \mathrm{~B}$ NMR (64 $\left.\mathrm{MHz}, \mathrm{CDCl}_{3}\right)$ : $\square$ 33.4; IR $\left(\mathrm{CH}_{2} \mathrm{Cl}_{2}\right.$ cast, $\left.\mathrm{cm}^{-1}\right)$ : 3070, 2931, 1718, 1622, 1589, 1348, 1146, 1111, 739, 702; HRMS (EI, $\left.m / z\right)$ Calcd for $\mathrm{C}_{29} \mathrm{H}_{40}{ }^{11} \mathrm{BSiO}_{4}\left(\mathrm{M}^{+}-(t)-\mathrm{Bu}\right)$ : 491.27890. Found: 491.27823.

\subsubsection{Ethyl (2E)-2-[(4,4,5,5-tetramethyl-1,3,2-dioxaborolan-2-yl)methyl]-3,5-dimethylhex-2-} enoate $1 \mathrm{n}$

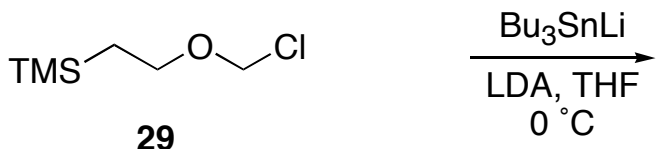

29

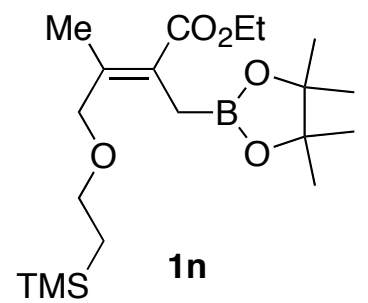<smiles>CCCC[SbH2]COCCS(C)(C)C</smiles>

30

1) BuLi, THF, $-78{ }^{\circ} \mathrm{C}$

2) $\mathrm{CuBr} \cdot \mathrm{SMe}_{2},-40{ }^{\circ} \mathrm{C}$<smiles>CC(C)(C)COCCS(C)(C)C</smiles>

31 
Stannane 30: ${ }^{18}$ A solution of diisopropylamine $(2.0 \mathrm{~mL}, 14 \mathrm{mmol})$ in THF $(25 \mathrm{~mL})$ at $0{ }^{\circ} \mathrm{C}$ was treated with BuLi $(1.65 \mathrm{M}, 7.5 \mathrm{~mL}, 12 \mathrm{mmol})$, stirred for $10 \mathrm{~min}$ and then treated dropwise with freshly distilled $\mathrm{Bu}_{3} \mathrm{SnH}(3.37 \mathrm{~g}, 11.6 \mathrm{mmol})$ and left to stir at this temperature. After 20 minutes, the resulting yellow solution was treated dropwise with SEM-Cl $29(5.04 \mathrm{~g}, 30.2 \mathrm{mmol})$, stirred for 5 minutes, and then poured into a mixture of hexane $(200 \mathrm{~mL})$ and $1 \mathrm{M} \mathrm{HCl} / \mathrm{brine}(100 \mathrm{~mL})$. The layers were separated and the organic layer was washed with $5 \% \mathrm{NaHCO}_{3(\mathrm{aq})}$, dried $\left(\mathrm{MgSO}_{4}\right)$ and concentrated to give the crude product. Distillation ( 0.1 torr, $150{ }^{\circ} \mathrm{C}$ bath) gave stannane 30 as a colourless oil (bp $135{ }^{\circ} \mathrm{C}$, $3.60 \mathrm{~g}, 8.54 \mathrm{mmol}, 74 \%)$. ${ }^{1} \mathrm{H}$ NMR (500 MHz, $\left.\mathrm{CDCl}_{3}\right): \square 3.68\left(\mathrm{~m}, J_{\mathrm{Sn}-\mathrm{H}}=6.8 \mathrm{~Hz}, 2 \mathrm{H}\right)$, $3.36(\mathrm{~m}, 2 \mathrm{H}), 1.49(\mathrm{~m}, 6 \mathrm{H}), 1.28(\mathrm{~m}, 6 \mathrm{H}), 0.88(\mathrm{~m}, 17 \mathrm{H}),-0.02(\mathrm{~s}, 9 \mathrm{H})$.

The cuprate 31 was prepared in analogy to the procedure of Overman and Paone. ${ }^{19}$ A flame dried flask under $\mathrm{Ar}$ was charged with a stir bar and $\mathrm{CuBr} \cdot \mathrm{SMe}_{2}(262 \mathrm{mg}$, $1.27 \mathrm{mmol}$ ) and immersed in a $-78{ }^{\circ} \mathrm{C}$ bath. In a second flask, a solution of stannane $\mathbf{3 0}$ $(1.02 \mathrm{~g}, 2.53 \mathrm{mmol})$ in THF $(3.8 \mathrm{~mL})$ under Ar was chilled to $-78{ }^{\circ} \mathrm{C}$ and treated with $\mathrm{BuLi}$ (1.65 M, $1.5 \mathrm{~mL}, 2.5 \mathrm{mmol})$. After $15 \mathrm{~min}$ this solution was transferred via canula to the flask containing $\mathrm{CuBr} \cdot \mathrm{SMe}_{2}$. The resulting pink mixture was then stirred at $-40{ }^{\circ} \mathrm{C}$ for $1 \mathrm{~h}$, causing a black mixture of the cuprate $\mathbf{3 1}$ to form. This mixture was then brought back to $-78{ }^{\circ} \mathrm{C}$, and the allylboronate 1n was prepared as described in Section 4.1.2, with ethyl 2-butynoate $(146 \mathrm{mg}, 1.30 \mathrm{mmol})$ and iodomethaneboronate $\mathbf{5 a}(0.75 \mathrm{~mL}, 3.9 \mathrm{mmol})$. The product was purified by flash chromatography $\left(25 \% \mathrm{Et}_{2} \mathrm{O} / \mathrm{Hexanes}, 91 \mathrm{~g}\right.$ silica) to give the desired allylboronate (134 mg, $0.348 \mathrm{mmol}, 28 \%$ ).

TLC (25\% Et $\left.2 \mathrm{O} / \mathrm{Hexane}, \mathrm{KMnO}_{4}\right): 0.28 ;{ }^{1} \mathrm{H} \mathrm{NMR}\left(500 \mathrm{MHz}, \mathrm{CDCl}_{3}\right): 04.16$ (q, $J=7.2 \mathrm{~Hz}, 2 \mathrm{H}), 3.98$ (s. 3H), 3.45 (m, 2H), 2.02 (s, 3H), 1.90 (s, $2 \mathrm{H}), 1.27$ (t, $J=7.1 \mathrm{~Hz}$, $3 \mathrm{H}), 1.20(\mathrm{~s}, 12 \mathrm{H}), 0.92(\mathrm{~m}, 2 \mathrm{H}),-0.01(\mathrm{~s}, 9 \mathrm{H}) ;{ }^{13} \mathrm{C} \mathrm{NMR}\left(100 \mathrm{MHz}, \mathrm{CDCl}_{3}\right): \square$ 169.1, $141.2,126.3,83.1,71.2,67.5,60.0,24.6,18.22,18.20,14.2,-1.4 ;{ }^{11} \mathrm{~B}$ NMR (64 MHz, $\left.\mathrm{CDCl}_{3}\right): \mathrm{Q} 32.7$; IR $\left(\mathrm{CH}_{2} \mathrm{Cl}_{2}\right.$ cast, $\left.\mathrm{cm}^{-1}\right): 2978,1717,1632,1349,1146$; HRMS (EI, $\mathrm{m} / z$ ) Calcd for $\mathrm{C}_{19} \mathrm{H}_{37}{ }^{11} \mathrm{BSiO}_{5}$ : 384.25034 . Found: 384.25140 .

(18) (a) Still, W. C. J. Am. Chem. Soc. 1978, 100, 1481-1486; (b) Hutchinson, D. K.; Fuchs, P. L. J. Am. Chem. Soc. 1987, 109, 4930-4939; (c) Buchwald, S. L.; Nielsen, R. B.; Dewan, J. C. Organometallics 1989, 8, 1593-1598.

(19) Overman, L.; Paone, D. V. J. Am. Chem. Soc. 2001, 123, 9465-9467. 


\subsubsection{Methyl (2E)-3-Phenyl-2-[(4,4,5,5-tetramethyl-1,3,2-dioxaborolan-2-yl)methyl]-but-2- enoate 10}

This compound was prepared according to the procedure in Section 4.1.2, with $\mathrm{CuBr} \cdot \mathrm{SMe}_{2}$ (242 mg, $\left.1.18 \mathrm{mmol}\right)$, MeLi (1.27 M in ether, $\left.1.8 \mathrm{~mL}, 2.3 \mathrm{mmol}\right)$, methyl 3-phenylpropynoate $\mathbf{3 g}(182 \mathrm{mg}, 1.14 \mathrm{mmol})$ and iodomethaneboronate $\mathbf{5 a}(865 \mathrm{mg}$, $3.23 \mathrm{mmol})$ to give the crude allylboronate as an oil $(244 \mathrm{mg})$. Purification by flash chromatography (25\% $\mathrm{Et}_{2} \mathrm{O} / \mathrm{Hexane}, 15 \mathrm{~g}$ silica) gave the desired allylboronate which contained some aromatic impurities (125 mg, $0.394 \mathrm{mmol}, 35 \%)$.

TLC (25\% Et 2 O/Hexane, $\mathrm{KMnO}_{4}$ ): 0.38; ${ }^{1} \mathrm{H}$ NMR (300 MHz, $\left.\mathrm{CDCl}_{3}\right): \square 7.3-7.0(\mathrm{~m}, 5 \mathrm{H})$, 3.35 (s, 3H), 2.07 (s, 3H), 2.00 (s, 2H), $1.24(\mathrm{~s}, 12 \mathrm{H}) ;{ }^{13} \mathrm{C} \mathrm{NMR}\left(125 \mathrm{MHz}, \mathrm{CDCl}_{3}\right)$ : $\mathrm{C} 170.3,144.3,142.5,127.7,126.8,126.5,125.3,83.2,51.0,24.8,22.3$; IR $\left(\mathrm{CH}_{2} \mathrm{Cl}_{2}\right.$ cast, $\mathrm{cm}^{-1}$ ): 2978, 1709, 1631, 1598, 1346, 1109, 765, 701; HRMS (EI, $\mathrm{m} / z$ ) Calcd for $\mathrm{C}_{18} \mathrm{H}_{25}{ }^{11} \mathrm{BO}_{4}$ : 316.18460. Found: 316.18460 .

\subsection{Allylboronates 1 from 2 equivalents of electrophile 5a}

\subsubsection{Methyl (2E)-2-[(4,4,5,5-tetramethyl-1,3,2-dioxaborolan-2-yl)methyl]-but-2-enoate 1a}

A slurry of $\mathrm{CuBr}: \mathrm{SMe}_{2}(211 \mathrm{mg}, 1.03 \mathrm{mmol})$ in $\mathrm{THF}(3 \mathrm{~mL})$ at $0{ }^{\circ} \mathrm{C}$ under Ar was treated with $\mathrm{MeLi}\left(1.45 \mathrm{M}\right.$ in $\left.\mathrm{Et}_{2} \mathrm{O}, 1.4 \mathrm{~mL}, 2.0 \mathrm{mmol}\right)$. When the clear, colourless solution had formed ( 10 $\mathrm{min})$ the flask was placed in an acetone $/ \mathrm{CO}_{2}$ bath and treated via canula with a $-78{ }^{\circ} \mathrm{C}$ solution of methyl propiolate $(86 \mathrm{mg}, 1.02 \mathrm{mmol})$ in THF $(0.5 \mathrm{~mL}+0.5 \mathrm{~mL}$ canula rinse). The resulting light brown mixture was stirred at $-78{ }^{\circ} \mathrm{C}$ for $1 \mathrm{~h}$ and then injected sequentially with iodomethaneboronate $5 \mathbf{a}(0.66 \mathrm{~g}, 2.5 \mathrm{mmol})$ and HMPA $(1.8 \mathrm{~mL}$, $10 \mathrm{mmol}$ ). After 5 minutes the mixture was brought to $\mathrm{rt}$, left to stir for $2 \mathrm{~h}$, and then the reaction was quenched with $\mathrm{NH}_{4} \mathrm{Cl}_{(\text {aq) }}(10 \mathrm{~mL})$. The layers were separated and the aqueous layer was extracted with ether $(4 \times 10 \mathrm{~mL})$. The combined organic layers were washed with water $(6 \times 10 \mathrm{~mL})$ and brine $(10 \mathrm{~mL})$, dried $\left(\mathrm{Na}_{2} \mathrm{SO}_{4}\right)$, and concentrated, yielding a yellow oil $(254 \mathrm{mg})$. Flash chromatography $\left(25 \% \mathrm{Et}_{2} \mathrm{O} /\right.$ Hexanes, $\left.15 \mathrm{~g} \mathrm{SiO}_{2}\right)$ gave the product as a colourless oil (140 mg, $0.611 \mathrm{mmol}, 60 \%)$.

TLC (25\% $\mathrm{Et}_{2} \mathrm{O} / \mathrm{Hexane}, \mathrm{KMnO}_{4}$ ): 0.31; ${ }^{1} \mathrm{H}$ NMR (300 MHz, $\mathrm{CDCl}_{3}$ ): 6.80 (qt, $J=7.0 \mathrm{~Hz}, 1.1 \mathrm{~Hz}, 1 \mathrm{H}), 3.68$ (s, 3H), 1.83 (br s, 2H), 1.75 (dt, $J=7.0 \mathrm{~Hz}, 0.8 \mathrm{~Hz}, 3 \mathrm{H}), 1.21$ (s, $12 \mathrm{H}) ;{ }^{13} \mathrm{C}$ NMR (50 MHz, $\mathrm{CDCl}_{3}$ ): $\square$ 168.3, 135.5, 129.9, 83.2, 51.5, 24.7, 14.5; ${ }^{11} \mathrm{~B}$ NMR (64 MHz, $\left.\mathrm{CDCl}_{3}\right)$ : $\square 32.6$; IR $\left(\mathrm{CH}_{2} \mathrm{Cl}_{2}\right.$ cast, $\left.\mathrm{cm}^{-1}\right)$ : 2978, 1712, 1651, 1145, 756; HRMS (EI, $m / z$ ) Calcd for $\mathrm{C}_{12} \mathrm{H}_{21}{ }^{11} \mathrm{BO}_{4}$ : 240.15329. Found: 240.15289 . 


\subsubsection{Methyl (2Z)-2-[(4,4,5,5-tetramethyl-1,3,2-dioxaborolan-2-yl)methyl]-3-ethylhept-2-enoate} $1 \mathrm{i}$

A slurry of $\mathrm{CuBr} \cdot \mathrm{SMe}_{2}(966.4 \mathrm{mg}, 4.70 \mathrm{mmol})$ in THF $(15 \mathrm{~mL})$ at $0{ }^{\circ} \mathrm{C}$ under $\mathrm{Ar}$ was treated with BuLi $(1.60 \mathrm{M}$ in Hexanes, $5.8 \mathrm{~mL}, 9.3 \mathrm{mmol})$. After 5 minutes, the dark mixture was placed in an acetone $/ \mathrm{CO}_{2}$ bath and treated via canula with a $-78{ }^{\circ} \mathrm{C}$ solution of ethyl 2-pentynoate $(592 \mathrm{mg}, 4.69 \mathrm{mmol})$ in THF $(2.5 \mathrm{~mL}+2.5 \mathrm{~mL}$ canula rinse). The resulting light brown mixture was stirred at $-78{ }^{\circ} \mathrm{C}$ for $1 \mathrm{~h}$ and then injected sequentially with iodomethaneboronate $5 \mathrm{a}(2.57 \mathrm{~g}, 9.59 \mathrm{mmol})$ and HMPA $(7.5 \mathrm{~mL}, 43 \mathrm{mmol})$. After 5 minutes the mixture was brought to room temperature, left to stir for $2 \mathrm{~h}$, and then reaction was quenched with $\mathrm{NH}_{4} \mathrm{Cl}_{\text {(aq) }}(25 \mathrm{~mL})$. The layers were separated and the aqueous layer was extracted with ether $(4 \times 25 \mathrm{~mL})$. The combined organic layers were washed with water $(6 \times 25 \mathrm{~mL})$ and brine $(25 \mathrm{~mL})$, dried $\left(\mathrm{Na}_{2} \mathrm{SO}_{4}\right)$, and concentrated, yielding a yellow oil $(254 \mathrm{mg})$. Flash chromatography $\left(25 \% \mathrm{Et}_{2} \mathrm{O} / \mathrm{Hexanes}, 114 \mathrm{~g} \mathrm{SiO}_{2}\right)$ gave the product as a colourless oil $(0.91 \mathrm{~g}, 2.80 \mathrm{mmol}, 60 \%)$.

TLC (25\% Et 2 O/Hexane, $\left.\mathrm{KMnO}_{4}\right): 0.44 ;{ }^{1} \mathrm{H}$ NMR $\left(500 \mathrm{MHz}, \mathrm{CDCl}_{3}\right): 4.13(\mathrm{q}, J=7.1 \mathrm{~Hz}$, $2 \mathrm{H}), 2.36(\mathrm{q}, J=7.4 \mathrm{~Hz}, 2 \mathrm{H}), 2.07(\mathrm{~m}, 2 \mathrm{H}), 1.83(\mathrm{br} \mathrm{s}, 2 \mathrm{H}), 1.42-1.38(\mathrm{~m}, 4 \mathrm{H}), 1.26(\mathrm{t}$, $J=7.1 \mathrm{~Hz}, 3 \mathrm{H}), 1.20(\mathrm{~s}, 12 \mathrm{H}), 1.02(\mathrm{t}, J=7.4 \mathrm{~Hz}, 3 \mathrm{H}), 0.89$ (t, $J=7.2 \mathrm{~Hz}, 3 \mathrm{H}) ;{ }^{13} \mathrm{C}$ NMR (125 MHz, $\mathrm{CDCl}_{3}$ ): $\square$ 169.2, 151.6, 123.3, 82.9, 59.7, 33.7, 30.0, 27.4, 24.7, 23.0, 14.2, 13.9, 13.3; ${ }^{11} \mathrm{~B}$ NMR $\left(64 \mathrm{MHz}, \mathrm{CDCl}_{3}\right)$ : $\square 32.9$; IR $\left(\mathrm{CH}_{2} \mathrm{Cl}_{2}\right.$ cast, $\left.\mathrm{cm}^{-1}\right): 2977,1714,1625$, 1348, 1147; HRMS (EI, $m / z$ ) Calcd for $\mathrm{C}_{18} \mathrm{H}_{33}{ }^{11} \mathrm{BO}_{4}$ : 324.24719. Found: 324.24757.

\section{Thermal reactions of 2-carboxyester 3,3-disubstituted allylboronates 1}

\subsection{Exo-methylene butyrolactones 2 from diastereoselective allylborations with 1}

\subsection{1 $\left(4 R^{\star}, 5 S^{\star}\right)-4-$ Methyl-3-methylene-5-phenyl-dihydro-furan-2-one 2a}

A solution of allylboronate 1a (298 mg, $1.24 \mathrm{mmol})$ and benzaldehyde $(66 \mathrm{mg}$, $0.63 \mathrm{mmol})$ in toluene $(2 \mathrm{~mL})$ were heated at $80^{\circ} \mathrm{C}$ for $64 \mathrm{~h}$. The mixture was then washed 
with $\mathrm{NH}_{4} \mathrm{Cl}_{(\mathrm{aq})}(10 \mathrm{~mL})$ and extracted with $\mathrm{Et}_{2} \mathrm{O}(3 \mathrm{x} 10 \mathrm{~mL})$. The combined extracts were washed with brine $(10 \mathrm{~mL})$, dried $\left(\mathrm{Na}_{2} \mathrm{SO}_{4}\right)$ and concentrated. The residue was purified by flash chromatography ( $25 \% \mathrm{Et}_{2} \mathrm{O} / \mathrm{Hexanes}, 10 \mathrm{~g}$ silica) to give a mixture of the lactone and the Ghydroxyester. This mixture was stirred overnight in $\mathrm{CH}_{2} \mathrm{Cl}_{2}(5 \mathrm{~mL})$ with a spatula tip of pTSA. The reaction was then quenched with $\mathrm{NaHCO}_{3(\mathrm{aq})}(5 \mathrm{~mL})$ and extracted with ether $(3 \times 5 \mathrm{~mL})$. The combined ether layers were washed with brine $(5 \mathrm{~mL})$, dried $\left(\mathrm{Na}_{2} \mathrm{SO}_{4}\right)$ and concentrated to give the desired lactone $(70 \mathrm{mg}, 0.37 \mathrm{mmol}, 60 \%)$.

${ }^{1} \mathrm{H}$ NMR (300 MHz, $\mathrm{CDCl}_{3}$ ): $\square 7.36(\mathrm{~m}, 3 \mathrm{H}), 7.18(\mathrm{~m}, 2 \mathrm{H}), 6.33$ (d, $\left.J=2.8 \mathrm{~Hz}, 1 \mathrm{H}\right), 5.59$ $(\mathrm{d}, J=8.1 \mathrm{~Hz}, 1 \mathrm{H}), 5.55(\mathrm{~d}, J=2.6 \mathrm{~Hz}, 1 \mathrm{H}), 3.42(\mathrm{~m}, 1 \mathrm{H}), 0.77(\mathrm{~d}, J=7.0 \mathrm{~Hz}, 3 \mathrm{H})$.

\subsection{2 rac-4,4-Dimethyl-3-methylene-5-phenyl-dihydro-furan-2-one 2b}

Allylboronate $1 \mathbf{b}(237 \mathrm{mg}, 0.885 \mathrm{mmol})$ and benzaldehyde $(62 \mathrm{mg}, 0.58 \mathrm{mmol})$ in $\mathrm{CH}_{2} \mathrm{Cl}_{2}(1 \mathrm{~mL})$ were refluxed under Ar. After $60 \mathrm{~h}$ the reaction was concentrated to give the crude products. Flash chromatography (13 g silica, 25-50\% $\mathrm{CH}_{2} \mathrm{Cl}_{2} / \mathrm{Hexanes}$ ) gave the product as a white solid (92 $\mathrm{mg}, 0.45 \mathrm{mmol}, 78 \%$ ). The compound could be further purified by Kugelrohr distillation $\left(0.1\right.$ torr, $\left.225^{\circ} \mathrm{C}\right)$ to give an analytically pure sample (83 mg, $0.41 \mathrm{mmol}, 71 \%$ ).

$\mathrm{mp}=48-50{ }^{\circ} \mathrm{C} ;{ }^{1} \mathrm{H}$ NMR $\left(300 \mathrm{MHz}, \mathrm{CDCl}_{3}\right)$ : $7.35(\mathrm{~m}, 3 \mathrm{H}), 7.22(\mathrm{~m}, 2 \mathrm{H}), 6.24(\mathrm{~s}, 1 \mathrm{H})$, $5.50(\mathrm{~s}, 1 \mathrm{H}), 5.14(\mathrm{~s}, 1 \mathrm{H}), 1.37(\mathrm{~s}, 3 \mathrm{H}), 0.72(\mathrm{~s}, 3 \mathrm{H}) ;{ }^{13} \mathrm{C} \mathrm{NMR}\left(75 \mathrm{MHz}, \mathrm{CDCl}_{3}\right)$ : 170.3 , 145.5, 135.7, 128.4, 128.3, 125.9, 120.1, 88.1, 43.6, 25.9, 24.3; IR $\left(\mathrm{CH}_{2} \mathrm{Cl}_{2}\right.$ cast, $\left.\mathrm{cm}^{-1}\right)$ : 3070, 2977, 1763, 1658, 1124, 751, 707; HRMS (EI, $m / z$ ) Calcd for $\mathrm{C}_{13} \mathrm{H}_{14} \mathrm{O}_{2}$ : 202.09938. Found: 202.09926; Anal. Calcd for $\mathrm{C}_{13} \mathrm{H}_{14} \mathrm{O}_{2}$ : C, 77.19; H, 6.99. Found: C, 76.94; H, 7.08.

\subsection{3 rac-5-(2-(tert-Butyldiphenylsilyloxy)ethyl)-4,4-dimethyl-3-methylene-dihydro-furan-2-one 2c}

3-(tert-Butyldiphenylsilyloxy)propanal $12(308 \mathrm{mg}, 1.15 \mathrm{mmol})$ and allylboronate $1 \mathbf{b}(289 \mathrm{mg}, 0.924 \mathrm{mmol})$ were stirred neat at room temperature for $12 \mathrm{~d}$. The crude mixture was then purified directly by flash chromatography $(22 \mathrm{~g}$ silica, $5 \% \mathrm{EtOAc} /$ Toluene) to give the product as a colourless oil (340 mg, $0.832 \mathrm{mmol}, 92 \%$ ). Kugelrohr distillation $\left(0.1\right.$ torr, $\left.225^{\circ} \mathrm{C}\right)$ afforded an analytical sample $(282 \mathrm{mg}, 0.690 \mathrm{mmol}$, $75 \%)$.

TLC (5\% EtOAc/Toluene, $\mathrm{KMnO}_{4}$ ): 0.40; ${ }^{1} \mathrm{H}$ NMR (300 MHz, $\mathrm{CDCl}_{3}$ ): 7.67 (m, 4H), $7.40(\mathrm{~m}, 6 \mathrm{H}), 6.14(\mathrm{~s}, 1 \mathrm{H}), 5.46(\mathrm{~m}, 1 \mathrm{H}), 4.30(\mathrm{dd}, J=10.4 \mathrm{~Hz}, 2.6 \mathrm{~Hz}, 1 \mathrm{H}), 3.84(\mathrm{~m}, 2 \mathrm{H})$, 
$1.72(\mathrm{~m}, 2 \mathrm{H}), 1.21$ (s, 3H), 1.04 (s, 12H); ${ }^{13} \mathrm{C}$ NMR (125 MHz, $\left.\mathrm{CDCl}_{3}\right)$ : $\square$ 170.1, 146.1, 135.4, 133.5, 129.7, 127.7, 119.1, 83.3, 60.2, 41.5, 32.8, 26.8, 24.9, 22.8, 19.1; IR $\left(\mathrm{CH}_{2} \mathrm{Cl}_{2}\right.$ cast, $\mathrm{cm}^{-1}$ ): 3070, 2960, 1767, 1650, 1600, 1296, 1194, 1112, 740, 702; HRMS (EI, $\mathrm{m} / z$ ) Calcd for $\mathrm{C}_{21} \mathrm{H}_{23} \mathrm{O}_{3} \mathrm{Si}$ : 351.14166. Found: 351.14269; Anal. Calcd for $\mathrm{C}_{21} \mathrm{H}_{23} \mathrm{O}_{3} \mathrm{Si}$ : C, 73.47; H, 7.91. Found: C, 73.00; H, 7.96.

\subsection{4 rac-4,4-Dimethyl-3-methylene-5-nonyl-dihydro-furan-2-one $2 \mathrm{~d}$}

A solution of $n$-decanal $(82 \mathrm{mg}, 0.52 \mathrm{mmol})$ and allylboronate $\mathbf{1 b}(178 \mathrm{mg}$, $0.631 \mathrm{mmol})$ in toluene $(0.5 \mathrm{~mL})$ was heated at $80{ }^{\circ} \mathrm{C}$ overnight. The solvent was removed and crude mixture was then purified by flash chromatography $(16 \mathrm{~g}$ silica, $5 \%$ EtOAc/Toluene) to give the product as a colourless oil (52 $\mathrm{mg}, 0.20 \mathrm{mmol}, 40 \%)$. Kugelrohr distillation $\left(0.1\right.$ torr, $\left.250{ }^{\circ} \mathrm{C}\right)$ afforded an analytical sample $(36 \mathrm{mg}, 0.14 \mathrm{mmol}$, $27 \%)$.

TLC (1\% EtOAc/Toluene, $\mathrm{KMnO}_{4}$ ): 0.37; ${ }^{1} \mathrm{H}$ NMR $\left(500 \mathrm{MHz}, \mathrm{CDCl}_{3}\right)$ : $\square 6.16$ (s, 1H); $5.44(\mathrm{~s}, 1 \mathrm{H}), 4.02(\mathrm{~m}, 1 \mathrm{H}), 1.64-1.47(\mathrm{~m}, 2 \mathrm{H}), 1.42-1.18(\mathrm{~m}, 14 \mathrm{H}), 1.20(\mathrm{~s}, 3 \mathrm{H}), 1.06(\mathrm{~s}$, $3 \mathrm{H}), 0.86(\mathrm{~m}, 3 \mathrm{H}) ;{ }^{13} \mathrm{C}$ NMR $\left(125 \mathrm{MHz}, \mathrm{CDCl}_{3}\right)$ : $\square$ 170.2, 146.2, 118.9, 87.4, 42.0, 31.9, 30.1, 29.56, 29.54, 29.53, 29.3, 26.4, 25.6, 22.8, 22.7, 14.2; $\mathrm{IR}\left(\mathrm{CH}_{2} \mathrm{Cl}_{2}\right.$ cast, $\left.\mathrm{cm}^{-1}\right): 2925$, 1769, 1661, 1108, 815; HRMS (EI, $m / z$ ) Calcd for $\mathrm{C}_{16} \mathrm{H}_{28} \mathrm{O}_{2}$ : 252.20892. Found: 252.20823; Anal. Calcd for $\mathrm{C}_{16} \mathrm{H}_{28} \mathrm{O}_{2}: \mathrm{C}, 76.12 ; \mathrm{H}, 11.20$. Found: C, 75.98; H, 11.00.

\subsection{5 $\left(4 R^{\star}, 5 S^{\star}\right)$-4-Ethyl-4-methyl-3-methylene-5-nonyl-dihydro-furan-2-one $2 \mathrm{e}$}

Decanal (161 mg, $1.03 \mathrm{mmol})$ and allylboronate $1 \mathrm{c}(435 \mathrm{mg}, 1.54 \mathrm{mmol})$ were stirred at room temperature under $\mathrm{N}_{2}$ in toluene $(1 \mathrm{~mL})$ for $48 \mathrm{~d}$. The solvent was then evaporated and the crude mixture was purified by flash chromatography (30 $\mathrm{g}$ silica, $5 \%$ EtOAc/Toluene) to give the product as a colourless oil (225 mg, $0.844 \mathrm{mmol}, 82 \%)$. The compound was then further purified by Kugelrohr distillation $\left(0.1\right.$ torr, $\left.200{ }^{\circ} \mathrm{C}\right)$ to give an analytically pure sample $(186 \mathrm{mg}, 0.700 \mathrm{mmol}, 68 \%)$.

TLC (5\% EtOAc/Toluene, $\mathrm{KMnO}_{4}$ ): 0.70; ${ }^{1} \mathrm{H} \mathrm{NMR}\left(500 \mathrm{MHz}, \mathrm{CDCl}_{3}\right)$ : $\square 6.21(\mathrm{~s}, 1 \mathrm{H})$, 5.39 (m, 1H), 4.16 (dd, $J=10.2 \mathrm{~Hz}, 2.9 \mathrm{~Hz}, 1 \mathrm{H}), 1.6-1.2$ (m, 18H), 1.08 (s, 3H), 0.85 (m, $6 \mathrm{H}) ;{ }^{13} \mathrm{C} \mathrm{NMR}\left(125 \mathrm{MHz}, \mathrm{CDCl}_{3}\right)$ : $\square$ 170.4, 144.1, 120.2, 88.0, 85.6, 45.1, 32.9, 31.9, 31.7, 29.6, 29.56, 29.55, 29.3, 26.2, 22.7, 20.3, 14.2, 8.4; IR $\left(\mathrm{CH}_{2} \mathrm{Cl}_{2}\right.$ cast, $\left.\mathrm{cm}^{-1}\right): 2924,1766$, 1660, 1109; HRMS (EI, $\mathrm{m} / \mathrm{z}$ ) Calcd for $\mathrm{C}_{17} \mathrm{H}_{30} \mathrm{O}_{2}: 266.2245$. Found: 266.22387; Anal. Calcd for $\mathrm{C}_{17} \mathrm{H}_{30} \mathrm{O}_{2}$ : C, 76.62; H, 11.37. Found: C, 76.67; H, 11.44. 


\subsection{6 $\left(4 R^{*}, 5 S^{*}\right)-4$-Ethyl-4-methyl-3-methylene-5-phenyl-dihydro-furan-2-one $2 f$}

Allylboronate 1c (413 mg, $1.46 \mathrm{mmol}$ ) and benzaldehyde (105 mg, $0.992 \mathrm{mmol}$ ) in toluene $(1 \mathrm{~mL})$ were stirred at room temperature under $\mathrm{N}_{2}$ for $48 \mathrm{~d}$. The mixture was then concentrated and the residue was purified by flash chromatography (30 $\mathrm{g}$ silica, $5 \% \mathrm{EtOAc} /$ Toluene) to give the product as a yellow oil (206 mg, $0.951 \mathrm{mmol}, 96 \%$ ). Kugelrohr distillation $\left(0.1\right.$ torr, $\left.250{ }^{\circ} \mathrm{C}\right)$ gave the product as a colourless oil $(191 \mathrm{mg}$, $0.883 \mathrm{mmol}, 89 \%)$.

TLC (5\% EtOAc/Toluene, UV/KMnO $)$ : 0.45; ${ }^{1} \mathrm{H}$ NMR (500 MHz, $\left.\mathrm{CDCl}_{3}\right): \square 7.32(\mathrm{~m}$, $3 \mathrm{H}), 7.13(\mathrm{~m}, 2 \mathrm{H}), 6.34(\mathrm{~s}, 1 \mathrm{H}), 5.44(\mathrm{~s}, 1 \mathrm{H}), 5.25(\mathrm{~s}, 1 \mathrm{H}), 1.70(\mathrm{~m}, 2 \mathrm{H}), 0.97(\mathrm{t}, J=7.3 \mathrm{~Hz}$, $3 \mathrm{H}), 0.71$ (s, 3H); ${ }^{13} \mathrm{C}$ NMR (125 MHz, $\mathrm{CDCl}_{3}$ ): $\square 170.6,142.8,136.9,128.3,128.2,126.0$, 125.9, 121.5, 86.3, 47.0, 33.6, 22.4, 8.5; IR $\left(\mathrm{CH}_{2} \mathrm{Cl}_{2}\right.$ cast, $\left.\mathrm{cm}^{-1}\right): 3034,2969,1765,1655$, 1604, 1106, 757, 701; HRMS (EI, $m / z$ ) Calcd for $\mathrm{C}_{14} \mathrm{H}_{16} \mathrm{O}_{2}$ : 216.11504. Found: 216.11490; Anal. Calcd for $\mathrm{C}_{14} \mathrm{H}_{16} \mathrm{O}_{2}$ : C, 77.73; H, 7.47. Found: C, 78.07; H, 7.77.

\subsection{7 (4R $\left.R^{\star}, 5 S^{\star}\right)$-4-Ethyl-5-(4-methoxyphenyl)- 4-methyl-3-methylene -dihydro-furan-2-one $2 \mathrm{~g}$}

Purified allylboronate 1c (326 mg, $1.15 \mathrm{mmol})$ and 4-methoxybenzaldehyde $(138 \mathrm{mg}, 1.01 \mathrm{mmol})$ in toluene $(1 \mathrm{~mL})$ were heated at $80{ }^{\circ} \mathrm{C}$ under Ar. After $60 \mathrm{~h}$ the mixture was concentrated and purified by flash chromatography $(30 \mathrm{~g}$ silica, $\left.25 \% \mathrm{Et}_{2} \mathrm{O} / \mathrm{Hexane}\right)$ to give the product as a colourless oil (179 $\left.\mathrm{mg}, 0.728 \mathrm{mmol}, 72 \%\right)$. Kugelrohr distillation $\left(0.1\right.$ torr, $\left.250{ }^{\circ} \mathrm{C}\right)$ gave an analytically pure sample $(176 \mathrm{mg}$, $0.713 \mathrm{mmol}, 70 \%)$.

TLC (5\% EtOAc/Toluene, UV/ $\mathrm{KMnO}_{4}$ ): 0.45; ${ }^{1} \mathrm{H}$ NMR (500 MHz, $\mathrm{CDCl}_{3}$ ): 77.00 (AB, $J=8.3 \mathrm{~Hz}, 2 \mathrm{H}), 6.80(\mathrm{AB}, J=8.8 \mathrm{~Hz}, 2 \mathrm{H}), 6.32(\mathrm{~s}, 1 \mathrm{H}), 5.39$ (s, 1H), 5.17 (s, $1 \mathrm{H}), 3.73$ (s, $3 \mathrm{H}), 1.65(\mathrm{~m}, 2 \mathrm{H}), 0.92(\mathrm{t}, J=7.3 \mathrm{~Hz}, 3 \mathrm{H}), 0.69(\mathrm{~s}, 3 \mathrm{H}) ;{ }^{13} \mathrm{C} \mathrm{NMR}\left(125 \mathrm{MHz}, \mathrm{CDCl}_{3}\right)$ : प 170.7, 159.6, 143.1, 129.0, 127.3, 121.4, 113.7, 86.3, 55.2, 46.9, 33.5, 22.1, 8.4; IR $\left(\mathrm{CH}_{2} \mathrm{Cl}_{2}\right.$ cast, $\left.\mathrm{cm}^{-1}\right): 2967,1765,1657,1612,1106,816$; HRMS (EI, $\mathrm{m} / \mathrm{z}$ ) Calcd for $\mathrm{C}_{15} \mathrm{H}_{18} \mathrm{O}_{3}: 246.12560$. Found: 246.12519; Anal. Calcd for $\mathrm{C}_{15} \mathrm{H}_{18} \mathrm{O}_{3}:$ C, 73.13; H, 7.38. Found: C, 72.82; H, 7.27. 


\subsection{8 $\left(4 R^{\star}, 5 S^{\star}\right)-4-E t h y l-4-m e t h y l-3-m e t h y l e n e-5-(4-n i t r o p h e n y l)-d i h y d r o-f u r a n-2-o n e ~ 2 h$}

Allylboronate 1c $(307 \mathrm{mg}, 1.09 \mathrm{mmol})$ and $p$-nitrobenzaldehyde $(95 \mathrm{mg}$, $0.63 \mathrm{mmol})$ in toluene $(1 \mathrm{~mL})$ were heated at $80{ }^{\circ} \mathrm{C}$ under Ar. After $5 \mathrm{~d}$ the reaction was concentrated and the residue was purified by flash chromatography (17 g silica, $5 \% \mathrm{EtOAc} /$ Toluene $)$ and then Kugelrohr distillation $\left(0.1\right.$ torr, $\left.225{ }^{\circ} \mathrm{C}\right)$ to give the product as an oil (133 mg, $0.508 \mathrm{mmol}, 81 \%)$.

TLC (5\% EtOAc/Toluene, UV/KMnO $\left.\left.)_{4}\right): 0.33 ;{ }^{1} \mathrm{H} \mathrm{NMR} \mathrm{(300} \mathrm{MHz,} \mathrm{CDCl}_{3}\right): \square 8.22(\mathrm{AB}$, $J=8.8 \mathrm{~Hz}, 2 \mathrm{H}), 7.35(\mathrm{AB}, J=8.3 \mathrm{~Hz}, 2 \mathrm{H}), 6.40(\mathrm{~s}, 1 \mathrm{H}), 5.51(\mathrm{~s}, 1 \mathrm{H}), 5.35(\mathrm{~s}, 1 \mathrm{H}), 1.75(\mathrm{~m}$, $2 \mathrm{H}), 1.01(\mathrm{t}, J=7.4 \mathrm{~Hz}, 3 \mathrm{H}), 0.72(\mathrm{~s}, 3 \mathrm{H}) ;{ }^{13} \mathrm{C} \mathrm{NMR}\left(75 \mathrm{MHz}, \mathrm{CDCl}_{3}\right)$ : $170.0,148.0$, 144.2, 141.9, 126.9, 123.7, 122.7, 84.6, 47.1, 33.3, 22.8, 8.5; IR $\left(\mathrm{CH}_{2} \mathrm{Cl}_{2}\right.$ cast, $\left.\mathrm{cm}^{-1}\right): 3083$, 2970, 1771, 1653, 1607, 1521, 1349, 1103; HRMS (EI, $m / z)$ Calcd for $\mathrm{C}_{14} \mathrm{H}_{15} \mathrm{NO}_{4}$ : 261.10010. Found: 261.10003; Anal. Calcd for $\mathrm{C}_{14} \mathrm{H}_{15} \mathrm{NO}_{4}$ : C, 64.35; H, 5.80; N, 5.36. Found: C, 63.98; H, 5.90; N, 5.13.

\subsection{9 $\left(4 R^{\star}, 5 S^{\star}\right)$-4-Butyl-4-methyl-3-methylene-5-(4-nitrophenyl)-dihydro-furan-2-one $2 \mathrm{i}$}

Allylboronate 1d (175 mg, $0.592 \mathrm{mmol})$ and $p$-nitrobenzaldehyde $(77 \mathrm{mg}$, $0.51 \mathrm{mmol})$ in toluene $(1 \mathrm{~mL})$ were heated at $80{ }^{\circ} \mathrm{C}$ under Ar overnight. The mixture was then concentrated and purified by flash chromatography (14 g silica, 25\% $\mathrm{Et}_{2} \mathrm{O} / \mathrm{Hexane}$, pre-absorption) to give the product as a colourless oil (112 $\mathrm{mg}, 0.387 \mathrm{mmol}, 76 \%$ ). Kugelrohr distillation $\left(0.1\right.$ torr, $\left.225^{\circ} \mathrm{C}\right)$ gave an analytically pure sample $(112 \mathrm{mg}$, $0.387 \mathrm{mmol}, 76 \%)$.

TLC (50\% $\left.\mathrm{Et}_{2} \mathrm{O} / \mathrm{Hexane}, \mathrm{UV} / \mathrm{KMnO}_{4}\right): 0.55 ;{ }^{1} \mathrm{H} \mathrm{NMR}\left(300 \mathrm{MHz}, \mathrm{CDCl}_{3}\right): \square 8.24(\mathrm{AB}$, $J=8.6 \mathrm{~Hz}, 2 \mathrm{H}), 7.50(\mathrm{AB}, J=8.6 \mathrm{~Hz}, 2 \mathrm{H}), 6.35(\mathrm{~s}, 1 \mathrm{H}), 5.54(\mathrm{~s}, 1 \mathrm{H}), 5.20(\mathrm{~s}, 1 \mathrm{H}), 1.38$ (s, 3H), 1.04 (m, 6H), 0.68 (m, 3H); ${ }^{13} \mathrm{C}$ NMR (75 MHz, $\left.\mathrm{CDCl}_{3}\right)$ : $\square$ 169.5, 147.9, 142.6, 142.3, 127.0, 123.6, 122.1, 87.1, 47.1, 34.4, 24.9, 22.7, 21.4, 13.7; IR $\left(\mathrm{CH}_{2} \mathrm{Cl}_{2}\right.$ cast, $\left.\mathrm{cm}^{-1}\right)$ : 2957, 1773, 1668, 1606, 1521, 1348; HRMS (EI, $m / z$ ) Calcd for $\mathrm{C}_{16} \mathrm{H}_{19} \mathrm{NO}_{4}$ : 289.13141. Found: 289.13071; Anal. Calcd for $\mathrm{C}_{16} \mathrm{H}_{19} \mathrm{NO}_{4}$ : C, 66.41; H, 6.63; N, 4.84. Found: C, 65.99; H, 6.72; N, 4.80 .

\subsubsection{0 (4R $\left.R^{*}, 5 R^{*}\right)-4-B u t y l-4-m e t h y l-3-m e t h y l e n e-5-(4-n i t r o p h e n y l)-d i h y d r o-f u r a n-2-o n e ~ 2 \mathrm{j}$}

Allylboronate 1e $(222 \mathrm{mg}, 0.717 \mathrm{mmol})$ and $p$-nitrobenzaldehyde $(79 \mathrm{mg}$, $0.52 \mathrm{mmol})$ in $\mathrm{CH}_{2} \mathrm{Cl}_{2}(1 \mathrm{~mL})$ were refluxed under Ar. After $48 \mathrm{~h}$ the reaction was concentrated to give the crude product. Flash chromatography (19 g silica, $5 \% \mathrm{EtOAc/Toluene)} \mathrm{gave} \mathrm{a} \mathrm{mixture} \mathrm{of} 3$ compounds. Further chromatography (10 g silica, 
$25 \% \mathrm{Et}_{2} \mathrm{O} /$ Hexanes) gave the product as a colourless oil (104 $\left.\mathrm{mg}, 0.360 \mathrm{mmol}, 69 \%\right)$. Kugelrohr distillation $\left(0.1\right.$ torr, $\left.225^{\circ} \mathrm{C}\right)$ gave an analytically pure sample $(101 \mathrm{mg}$, $0.349 \mathrm{mmol}, 67 \%)$.

TLC (50\% Et $\left.{ }_{2} \mathrm{O} / \mathrm{Hexanes,} \mathrm{UV} / \mathrm{KMnO}_{4}\right): 0.35 ;{ }^{1} \mathrm{H}$ NMR $\left(300 \mathrm{MHz}, \mathrm{CDCl}_{3}\right): \square 8.22(\mathrm{AB}$, $J=8.6 \mathrm{~Hz}, 2 \mathrm{H}), 7.34(\mathrm{AB}, J=8.4 \mathrm{~Hz}, 2 \mathrm{H}), 6.38(\mathrm{~s}, 1 \mathrm{H}), 5.50(\mathrm{~s}, 1 \mathrm{H}), 5.36(\mathrm{~s}, 1 \mathrm{H}), 1.67$ (m, 2H), $1.36(\mathrm{~m}, 2 \mathrm{H}), 0.94(\mathrm{~m}, 3 \mathrm{H}), 0.72(\mathrm{~s}, 3 \mathrm{H}) ;{ }^{13} \mathrm{C} \mathrm{NMR}\left(75 \mathrm{MHz}, \mathrm{CDCl}_{3}\right)$ : $170.0,147.9$, 144.2, 142.2, 126.9, 123.7, 122.5, 84.9, 46.8, 40.6, 26.2, 23.2, 23.0, 13.9; IR $\left(\mathrm{CH}_{2} \mathrm{Cl}_{2}\right.$ cast, $\left.\mathrm{cm}^{-1}\right):$ 3083, 2952, 1771, 1659, 1607, 1522, 1349, 1105; HRMS (EI, $m / z$ ) Calcd for $\mathrm{C}_{11} \mathrm{H}_{18} \mathrm{O}_{2}$ : 182.13068. Found: 182.13093; Anal. Calcd for $\mathrm{C}_{16} \mathrm{H}_{19} \mathrm{NO}_{4}$ : C, 77.73; H, 7.47. Found: C, 78.07; H, 7.77.

\subsubsection{1 $\left(4 R^{\star}, 5 R^{\star}\right)$-5-(4-Methoxyphenyl)-4-methyl-3-methylene-4-(2-methylpropyl)- dihydro-furan- 2-one $2 k$}

A solution of allylboronate $\mathbf{1 f}(280 \mathrm{mg}, 0.902 \mathrm{mmol})$ and $p$-anisaldehyde $(111 \mathrm{mg}$, $0.813 \mathrm{mmol})$ in toluene $(1 \mathrm{~mL})$ were heated at $110{ }^{\circ} \mathrm{C}$ for $24 \mathrm{~h}$. The solvent was then removed and the residue purified by flash chromatography $\left(25 \% \mathrm{Et}_{2} \mathrm{O} / \mathrm{Hexanes,} 20 \mathrm{~g}\right.$ silica) to give the title lactone (145 $\mathrm{mg}, 0.528 \mathrm{mmol}, 65 \%$ ).

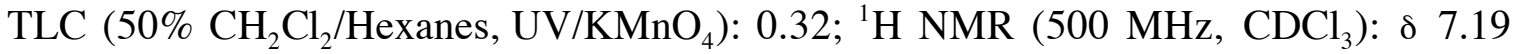
$(\mathrm{AB}, J=8.9 \mathrm{HZ}, 2 \mathrm{H}), 6.89(\mathrm{AB}, J=8.4 \mathrm{~Hz}, 2 \mathrm{H}), 6.27(\mathrm{~d}, J=0.6 \mathrm{~Hz}, 1 \mathrm{H}), 5.51$ (d, $J=0.5 \mathrm{~Hz}, 1 \mathrm{H}), 5.03(\mathrm{~s}, 1 \mathrm{H}), 3.81(\mathrm{~d}, J=0.6 \mathrm{~Hz}, 3 \mathrm{H}), 1.45(\mathrm{~m}, 1 \mathrm{H}), 1.33$ (s, 3H), 1.14 (dd, $J=14.4 \mathrm{~Hz}, 4.6 \mathrm{~Hz}, 1 \mathrm{H}), 0.70(\mathrm{~m}, 7 \mathrm{H}) ;{ }^{13} \mathrm{C} \mathrm{NMR}\left(125 \mathrm{MHz}, \mathrm{CDCl}_{3}\right): \square 170.3,159.5$, $144.4,127.5,126.6,120.5,113.7,88.7,55.3,47.7,42.9,25.4,25.0,23.5,22.3 ; \mathrm{IR}\left(\mathrm{CH}_{2} \mathrm{Cl}_{2}\right.$ cast, $\mathrm{cm}^{-1}$ ) 2958, 1770, 1659, 1613, 1386, 1366, 1251, 817; HRMS (EI, $m / z$ ) Calcd for $\mathrm{C}_{17} \mathrm{H}_{22} \mathrm{O}_{3}: 274.15689$. Found: 274.15618; Calcd for $\mathrm{C}_{17} \mathrm{H}_{22} \mathrm{O}_{3}: \mathrm{C}, 74.41 ; \mathrm{H}, 8.10$. Found: C, 74.06; H, 8.11.

\subsubsection{2 rac-3-(3-Butenyl)-4-methyl-5-(4-methoxyphenyl)-5H-furan-2-one 2 I}

A solution of allylboronate 1k (143 $\mathrm{mg}, 0.511 \mathrm{mmol})$ and $p$-anisaldehyde $(64 \mathrm{mg}$, $0.47 \mathrm{mmol})$ in toluene $(1 \mathrm{~mL})$ were heated at $110{ }^{\circ} \mathrm{C}$ for $16 \mathrm{~h}$. The solvent was then removed and the residue purified by flash chromatography $\left(25 \% \mathrm{Et}_{2} \mathrm{O} / \mathrm{Hexanes}, 23 \mathrm{~g}\right.$ silica) to give the title lactone as its [3+3] rearranged product (73 $\mathrm{mg}, 0.282 \mathrm{mmol}, 60 \%)$. Kugelrohr distillation $\left(250{ }^{\circ} \mathrm{C}, 0.1\right.$ torr $)$ gave an analytical sample $(59.2 \mathrm{mg}, 0.23 \mathrm{mmol}$, $48 \%)$.

TLC (25\% Et $\left.{ }_{2} \mathrm{O} / \mathrm{Hexanes} \mathrm{UV} / \mathrm{KMnO}_{4}\right): 0.17 ;{ }^{1} \mathrm{H} \mathrm{NMR}\left(500 \mathrm{MHz}, \mathrm{CDCl}_{3}\right): \square 7.09(\mathrm{AB}$, 
$J=8.7 \mathrm{~Hz}, 2 \mathrm{H}), 6.87(\mathrm{AB}, J=8.6 \mathrm{~Hz}, 2 \mathrm{H}), 5.78(\mathrm{dtt}, J=18.0 \mathrm{~Hz}, 9.0 \mathrm{~Hz}, 7.7 \mathrm{~Hz}, 1 \mathrm{H}), 5.53$ (s, 1H), $5.04(\mathrm{dd}, J=17.1 \mathrm{~Hz}, 1.4 \mathrm{~Hz}, 1 \mathrm{H}), 4.98(\mathrm{~d}, J=10.1 \mathrm{~Hz}, 1 \mathrm{H}), 3.79(\mathrm{~s}, 3 \mathrm{H}), 2.40(\mathrm{~m}$, 2H), $2.32(\mathrm{~m}, 2 \mathrm{H}), 1.77$ (s, 3H).; ${ }^{13} \mathrm{C}$ NMR (125 MHz, $\left.\mathrm{CDCl}_{3}\right)$ : $\mathrm{c}$ 174.0, 160.2, 159.7, 137.2, 128.3, 126.7, 126.4, 115.8, 114.3, 84.8, 55.3, 32.1, 23.2, 12.4; IR $\left(\mathrm{CH}_{2} \mathrm{Cl}_{2}\right.$ cast, $\left.\mathrm{cm}^{-1}\right)$ : 3071, 2931, 1773, 1660, 1607, 1105, 823, 741, 702; HRMS (EI, $m / z$ ) Calcd for $\mathrm{C}_{16} \mathrm{H}_{18} \mathrm{O}_{3}$ : 258.12558. Found:. 258.12529.

\subsubsection{3 $\left(4 R^{\star}, 5 S^{\star}\right)$-4-Methyl-3-methylene-5-(4-nitrophenyl)-4-phenyl-dihydro-furan-2-one $2 \mathrm{~m}$}

Allylboronate $11(125 \mathrm{mg}, 0.394 \mathrm{mmol})$ and 4-nitrobenzaldehyde $(62 \mathrm{mg}$, $0.41 \mathrm{mmol})$ in toluene $(1 \mathrm{~mL})$ were heated at $80{ }^{\circ} \mathrm{C}$ under Ar for $64 \mathrm{~h}$. The mixture was then concentrated and purified by flash chromatography (25 g silica, $50 \% \mathrm{CH}_{2} \mathrm{Cl}_{2} / \mathrm{Hexane}$ ) to give the solid product ( $32 \mathrm{mg}, 0.10 \mathrm{mmol}, 26 \%$ ).

${ }^{1} \mathrm{H}$ NMR $\left(500 \mathrm{MHz}, \mathrm{CDCl}_{3}\right)$ : $\square 8.15(\mathrm{AB}, J=8.9 \mathrm{~Hz}, 2 \mathrm{H}), 7.43(\mathrm{~m}, 2 \mathrm{H}), 7.38(\mathrm{~m}, 1 \mathrm{H})$, $7.33(\mathrm{~m}, 2 \mathrm{H}), 7.16(\mathrm{AB}, J=8.6 \mathrm{~Hz}, 2 \mathrm{H}), 6.43$ (s, 1H), 5.67 (s, 1H), 5.41 (s, 1H), 1.09 (s, $3 \mathrm{H}) ;{ }^{13} \mathrm{C}$ NMR (125 MHz, $\mathrm{CDCl}_{3}$ ): $\square$ 168.7, 147.8, 145.7, 142.0, 141.2, 129.0, 128.0, 127.4, 126.3, 123.8, 123.5, 87.7, 51.8, 22.1; Anal. Calcd for $\mathrm{C}_{18} \mathrm{H}_{15} \mathrm{NO}_{4}$ : C, 69.88; H, 4.90; N, 4.53. Found: C, 69.28; H, 4.99; N, 4.36.

\subsubsection{4 $\left(4 R^{\star}, 5 R^{\star}\right)$-4-(tert-Butyldiphenylsilyloxymethyl)-4-methyl-3-methylene-5-(4-nitrophenyl)- dihydro-furan-2-one $2 n$}

A solution of allylboronate $10(308 \mathrm{mg}, 0.605 \mathrm{mmol})$ and 4-nitrobenzaldehyde (90 mg, $0.59 \mathrm{mmol}$ ) in toluene $(1 \mathrm{~mL})$ were heated at $80{ }^{\circ} \mathrm{C}$ for $64 \mathrm{~h}$. The solvent was then removed and the residue purified by flash chromatography $\left(50 \% \mathrm{CH}_{2} \mathrm{Cl}_{2} / \mathrm{Hexanes,} 25 \mathrm{~g}\right.$ silica, pre-absorption) to give the title lactone ( $223 \mathrm{mg}, 0.444 \mathrm{mmol}, 75 \%)$. This compound was not sufficiently volatile for Kugelrohr distillation.

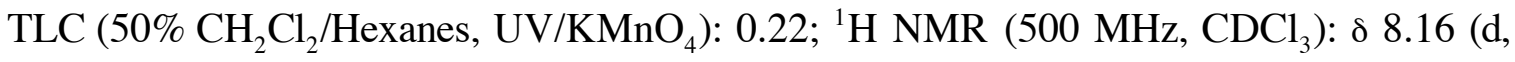
$J=8.9 \mathrm{~Hz}, 2 \mathrm{H}), 7.63(\mathrm{~m}, 4 \mathrm{H}), 7.44(\mathrm{~m}, 6 \mathrm{H}), 7.23(\mathrm{~d}, J=7.9 \mathrm{~Hz}, 2 \mathrm{H}), 6.39(\mathrm{~s}, 1 \mathrm{H}), 5.64(\mathrm{~s}$, $1 \mathrm{H}), 5.48(\mathrm{~s}, 1 \mathrm{H}), 3.72(\mathrm{AB}, J=10.2 \mathrm{~Hz}, 1 \mathrm{H}), 3.54(\mathrm{AB}, J=10.2 \mathrm{~Hz}, 1 \mathrm{H}), 1.10(\mathrm{~s}, 9 \mathrm{H})$, 0.67 (s, 3H); ${ }^{13} \mathrm{C}$ NMR (125 MHz, $\mathrm{CDCl}_{3}$ ): $\mathrm{Q}$ 169.4, 147.8, 144.2, 140.1, 135.7, 135.6, $132.4,132.3,130.2,130.1,128.0,127.9,126.9,123.6,123.5,82.7,69.6,48.8,27.0,20.2$, 19.4; IR $\left(\mathrm{CH}_{2} \mathrm{Cl}_{2}\right.$ cast, $\left.\mathrm{cm}^{-1}\right): 3071,2931,1773,1660,1607,1105,823,741,702$; HRMS (EI, $\mathrm{m} / z$ ) Calcd for $\mathrm{C}_{25} \mathrm{H}_{22} \mathrm{NO}_{5} \mathrm{Si}\left(\mathrm{M}^{+}{ }^{-} \mathrm{Bu}\right)$ : 444.12674. Found: 444.12487. 


\subsection{Enantioselective allylborations - Single auxiliary approach}

\subsubsection{Chiral alkynoates 10}

\subsubsection{2-Butynoic acid, $(R)$-pantolactone ester 10a}

This compound was prepared in analogy to the procedure of Koh and co-workers. ${ }^{20}$ A solution of 2-butynoic acid 8 (470 $\mathrm{mg}, 5.59 \mathrm{mmol}),(R)$-pantolactone 9a (742 $\mathrm{mg}$, $5.70 \mathrm{mmol})$, DCC (1.33 g, $6.45 \mathrm{mmol})$ and DMAP (105 $\mathrm{mg}, 0.857 \mathrm{mmol}$ ) in $\mathrm{CH}_{2} \mathrm{Cl}_{2}$ $(75 \mathrm{~mL})$ was stirred at room temperature under Ar for $2 \mathrm{~d}$. The resulting orange mixture was diluted with water $(100 \mathrm{~mL})$, the layers were separated, and the aqueous phase was extracted with $\mathrm{CH}_{2} \mathrm{Cl}_{2}(3 \times 25 \mathrm{~mL})$. The combined organic layers were washed with $\mathrm{NaHCO}_{3(\mathrm{aq})}(2 \times 25 \mathrm{~mL})$ and brine $(50 \mathrm{~mL})$, dried $\left(\mathrm{Na}_{2} \mathrm{SO}_{4}\right)$ and concentrated to give the

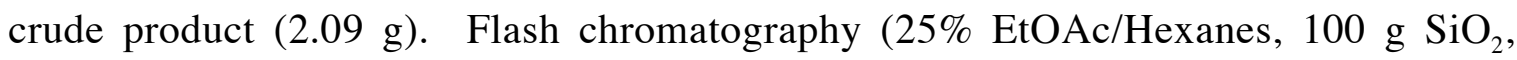
pre-absorption) gave the pure product as an oil (0.98 g, $5.0 \mathrm{mmol}, 89 \%)$. Kugelrohr distillation of a small amount $\left(0.1\right.$ torr, $\left.150{ }^{\circ} \mathrm{C}\right)$ gave an analytical sample which solidified over time.

mp 65-67 ${ }^{\circ} \mathrm{C}$; $[\square]_{\mathrm{D}}^{26}(+) 6.5$ (c 3.19, $\left.\mathrm{CHCl}_{3}\right) ;{ }^{1} \mathrm{H}$ NMR $\left(300 \mathrm{MHz}, \mathrm{CDCl}_{3}\right): \square 5.36(\mathrm{~s}, 1 \mathrm{H})$, $4.06(\mathrm{AB}, J=9.0 \mathrm{~Hz}, 1 \mathrm{H}), 3.98(\mathrm{AB}, J=9.1 \mathrm{~Hz}, 1 \mathrm{H}), 2.01(\mathrm{~s}, 3 \mathrm{H}), 1.20(\mathrm{~s}, 3 \mathrm{H}), 1.12$ (s, $3 \mathrm{H}) ;{ }^{13} \mathrm{C} \mathrm{NMR}\left(125 \mathrm{MHz}, \mathrm{CDCl}_{3}\right.$ ): $\square$ 171.5, 152.3, 88.7, 76.2, 76.0, 71.3, 40.3, 23.0, 19.8, 3.9; IR $\left(\mathrm{CH}_{2} \mathrm{Cl}_{2}\right.$ cast, $\left.\mathrm{cm}^{-1}\right)$ : 2970, 2236, 1792, 1720, 1248, 1096; HRMS (EI, $\left.m / z\right)$ Calcd for $\mathrm{C}_{10} \mathrm{H}_{12} \mathrm{O}_{4}, 196.07356$, found 196.07383; Anal. Calcd for $\mathrm{C}_{10} \mathrm{H}_{12} \mathrm{O}_{4}$ : C, 61.2; H, 6.2. Found: C, 60.94; H, 6.21.

\subsubsection{2-Butynoic acid, (-)-borneol ester 10b}

This compound was made in analogy to the procedure of Fonquerna and co-workers. ${ }^{21}$ A solution of 2-butynoic acid 8 (500 mg, $\left.5.95 \mathrm{mmol}\right)$ and (-)-borneol 9b (922 mg, $5.98 \mathrm{mmol})$ in $\mathrm{CH}_{2} \mathrm{Cl}_{2}(7.5 \mathrm{~mL})$ at $0{ }^{\circ} \mathrm{C}$ under $\mathrm{Ar}$ was treated dropwise with a solution of DCC (1.26 g, $6.11 \mathrm{mmol})$ and DMPA $(8.7 \mathrm{mg}, 0.071 \mathrm{mmol})$ in $\mathrm{CH}_{2} \mathrm{Cl}_{2}$ $(7.5 \mathrm{~mL})$. The resulting mixture was stirred at $0{ }^{\circ} \mathrm{C}$ for $4.5 \mathrm{~h}$ and then at room temperature for $2 \mathrm{~h}$, after which time the mixture was filtered. The residue was washed with $\mathrm{CH}_{2} \mathrm{Cl}_{2}$ $(6 \times 5 \mathrm{~mL})$ and the combined filtrates were concentrated to give an orange slush $(1.45 \mathrm{~g})$. Flash chromatography $\left(5 \% \mathrm{Et}_{2} \mathrm{O} /\right.$ Hexanes, $63 \mathrm{~g}$ silica, pre-absorption) gave the pure

(21) Foquerna, S.; Moyano, A.; Pericàs, M. A.; Riera, A. Tetrahedron 1995, 51, 4239-4254. 
product as a colourless oil which solidified under vacuum (1.06 g, $4.78 \mathrm{mmol}, 80 \%)$. Kugelrohr distillation of a small amount of the product $\left(100{ }^{\circ} \mathrm{C}, 0.1\right.$ torr) gave an analytical sample.

mp 57-59 ${ }^{\circ} \mathrm{C}$; [व] ${ }_{\mathrm{D}}^{26}$ (-) 43.5 (c 1.14, $\left.\mathrm{CHCl}_{3}\right) ;{ }^{1} \mathrm{H}$ NMR $\left(300 \mathrm{MHz}, \mathrm{CDCl}_{3}\right): \square 4.93(\mathrm{~m}, 1 \mathrm{H})$, $2.34(\mathrm{~m}, 1 \mathrm{H}), 1.97(\mathrm{~m}, 4 \mathrm{H}), 1.78(\mathrm{~m}, 2 \mathrm{H}), 1.34(\mathrm{~m}, 2 \mathrm{H}), 1.01(\mathrm{dd}, J=13.8 \mathrm{~Hz}, 3.5 \mathrm{~Hz}, 1 \mathrm{H})$, 0.87 (s, 3H), 0.85 (s, 3H), 0.83 (s, 3H); ${ }^{13} \mathrm{C}$ NMR (50 MHz, $\mathrm{CDCl}_{3}$ ): $\square$ 154.1, 84.8, 81.5, 72.8, 48.8, 47.8, 44.7, 36.4, 27.9, 27.0, 19.6, 18.7, 13.4, 3.7; IR $\left(\mathrm{CH}_{2} \mathrm{Cl}_{2}\right.$ cast, $\left.\mathrm{cm}^{-1}\right): 2955$, 2244, 1708, 1261; HRMS (EI, $m / z$ ) Calcd for $\mathrm{C}_{14} \mathrm{H}_{20} \mathrm{O}_{2}, 220.14633$, found 220.14604; Anal. Calcd for $\mathrm{C}_{14} \mathrm{H}_{20} \mathrm{O}_{2}$ : C, 76.31; H, 9.17. Found: C, 76.06; H, 9.17.

\subsubsection{2-Butynoic acid, $(R)$-1-phenylethanol ester 10c}

This compound was prepared according to the procedure described in Section 5.2.1.2, with 2-butynoic acid 8 (0.786 g, $9.35 \mathrm{mmol}),(R)$-1-phenylethanol 9c $(1.02 \mathrm{~g}$, $8.33 \mathrm{mmol})$, DCC (1.90 g, $9.22 \mathrm{mmol})$ and DMAP (51 $\mathrm{mg}, 0.42 \mathrm{mmol})$. Work up gave a dark orange slush $(1.98 \mathrm{~g})$. Flash chromatography $\left(5 \% \mathrm{Et}_{2} \mathrm{O} / \mathrm{Hexanes,} 100 \mathrm{~g}\right.$ silica, pre-absorption) gave the product $(1.24 \mathrm{~g}, 6.59 \mathrm{mmol}, 79 \%)$ as a colourless oil.

TLC (5\% Et ${ }_{2} \mathrm{O} / \mathrm{Hexanes,} \mathrm{UV} / \mathrm{KMnO}_{4}$ ): 0.24; [व] ${ }_{\mathrm{D}}^{26}(+) 4.55$ (c 4.46, $\left.\mathrm{CHCl}_{3}\right) ;{ }^{1} \mathrm{H}$ NMR $\left(300 \mathrm{MHz}, \mathrm{CDCl}_{3}\right): \square 7.36(\mathrm{~m}, 5 \mathrm{H}), 5.32(\mathrm{q}, J=6.6 \mathrm{~Hz}, 1 \mathrm{H}), 1.96(\mathrm{~s}, 3 \mathrm{H}), 1.56(\mathrm{~d}$, $J=6.7 \mathrm{~Hz}, 3 \mathrm{H}) ;{ }^{13} \mathrm{C} \mathrm{NMR}\left(75 \mathrm{MHz}, \mathrm{CDCl}_{3}\right): \square 152.9,140.7,128.5,128.1,126.1,85.5$, 73.9, 72.6, 22.0, 3.7; IR $\left(\mathrm{CH}_{2} \mathrm{Cl}_{2}\right.$ cast, $\left.\mathrm{cm}^{-1}\right)$ : 2982, 2240, 1707, 1253, 750, 699; HRMS (EI, $m / z$ ) Calcd for $\mathrm{C}_{12} \mathrm{H}_{12} \mathrm{O}_{2}, 188.08372$, found 188.08347; Anal. Calcd for $\mathrm{C}_{12} \mathrm{H}_{12} \mathrm{O}_{2}$ : C, 76.61; H, 6.44. Found: C, 76.63; H, 6.45.

\subsubsection{2-Butynoic acid, (-)-menthol ester 10d}

This compound was prepared according to the procedure described in Section 5.2.1.2, with 2-butynoic acid 8 (0.516 $\mathrm{mg}, 6.14 \mathrm{mmol})$, (-)-menthol 9d (948 $\mathrm{mg}$, $6.07 \mathrm{mmol})$, DCC $(1.24 \mathrm{~g}, 6.00 \mathrm{mmol})$ and DMAP $(17.8 \mathrm{mg}, 0.146 \mathrm{mmol})$ in $\mathrm{CH}_{2} \mathrm{Cl}_{2}$ $(7.5 \mathrm{~mL}$ ) except that the reaction was left to stir at room temperature for $7 \mathrm{~d}$. Work up gave a dark orange slush $(2.57 \mathrm{~g},>100 \%)$. Flash chromatography $\left(5 \% \mathrm{Et}_{2} \mathrm{O} /\right.$ Hexanes, $70 \mathrm{~g}$ silica, pre-absorption) gave the pure product as a colourless oil which solidified under vacuum $(1.05 \mathrm{~g}, 4.74 \mathrm{mmol}, 78 \%)$. Kugelrohr distillation of a small amount of the product $\left(100{ }^{\circ} \mathrm{C}, 0.1\right.$ torr) gave an analytical sample.

mp 36-38 ${ }^{\circ} \mathrm{C}$; $\left[\mathrm{C}^{26}{ }_{\mathrm{D}}\right.$ (-) $65.8\left(\mathrm{c} 1.07, \mathrm{CH}_{2} \mathrm{Cl}_{2}\right) ;{ }^{1} \mathrm{H}$ NMR (300 MHz, $\left.\mathrm{CDCl}_{3}\right): \square 4.74$ (dt, $J=10.9 \mathrm{~Hz}, 4.5 \mathrm{~Hz}, 1 \mathrm{H}), 1.94(\mathrm{~m}, 4 \mathrm{H}), 1.65(\mathrm{~m}, 2 \mathrm{H}), 1.41(\mathrm{~m}, 2 \mathrm{H}), 1.05(\mathrm{~m}, 2 \mathrm{H}), 0.88(\mathrm{~m}$, 
$8 \mathrm{H}), 0.74(\mathrm{~d}, J=7.0 \mathrm{~Hz}, 3 \mathrm{H}) ;{ }^{13} \mathrm{C} \mathrm{NMR}\left(125 \mathrm{MHz}, \mathrm{CDCl}_{3}\right)$ : $\square 153.3,84.8,75.8,72.7,46.8$, 40.6, 34.0, 31.3, 26.1 , 23.2, 21.9, 20.6, 16.1, 3.7; $\mathrm{IR}\left(\mathrm{CH}_{2} \mathrm{Cl}_{2}\right.$ cast, $\left.\mathrm{cm}^{-1}\right)$ : 2956, 2243, 1706, 1455, 1387, 1256; HRMS (EI, $m / z$ ) Calcd for $\mathrm{C}_{14} \mathrm{H}_{22} \mathrm{O}_{2}, 222.16199$, found 222.16378.

\subsubsection{2-Butynoic acid, (-)-8-phenylmenthol ester 10e}

A solution of 2-butynoic acid $8(0.54 \mathrm{~g}, 6.4 \mathrm{mmol})$ and (-)-8-phenylmenthol 9e (1.01 g, $4.36 \mathrm{mmol})$ in $\mathrm{CH}_{2} \mathrm{Cl}_{2}(4 \mathrm{~mL})$ at $-78{ }^{\circ} \mathrm{C}$ under Ar was treated dropwise with a solution of DCC (1.42 g, $6.89 \mathrm{mmol})$ and DMAP (100 mg, $0.824 \mathrm{mmol})$ in $\mathrm{CH}_{2} \mathrm{Cl}_{2}$ $(4 \mathrm{~mL})$. The resulting mixture was left to slowly come to room temperature overnight. The mixture was then filtered, the residue was washed with $\mathrm{CH}_{2} \mathrm{Cl}_{2}(6 \times 5 \mathrm{~mL})$ and the combined filtrates were concentrated to give an orange slush $(2.17 \mathrm{~g})$. Flash chromatography (5\% $\mathrm{Et}_{2} \mathrm{O} / \mathrm{Hexanes,} 100 \mathrm{~g}$ silica, pre-absorption) gave the pure product as a colourless oil $(1.19 \mathrm{~g}, 3.99 \mathrm{mmol}, 91 \%)$, which was further purified by Kugelrohr distillation $\left(250{ }^{\circ} \mathrm{C}\right.$, 0.1 torr) to give the pure product as a white solid $(1.15 \mathrm{~g}, 3.87 \mathrm{mmol}, 89 \%)$.

$[\square]^{26}{ }_{\mathrm{D}}(+) 9.7$ (c 2.62, $\left.\mathrm{CHCl}_{3}\right) ;{ }^{1} \mathrm{H}$ NMR $\left(500 \mathrm{MHz}, \mathrm{CDCl}_{3}\right)$ : $\mathrm{\square} 7.30(\mathrm{~m}, 4 \mathrm{H}), 7.16(\mathrm{~m}, 1 \mathrm{H})$, $4.85(\mathrm{dt}, J=10.8 \mathrm{~Hz}, 4.6 \mathrm{~Hz}, 1 \mathrm{H}), 1.97(\mathrm{~m}, 2 \mathrm{H}), 1.89(\mathrm{~s}, 3 \mathrm{H}), 1.54(\mathrm{~m}, 2 \mathrm{H}), 1.42(\mathrm{~m}, 1 \mathrm{H})$, $1.34(\mathrm{~s}, 3 \mathrm{H}), 1.25(\mathrm{~s}, 3 \mathrm{H}), 1.02(\mathrm{~m}, 2 \mathrm{H}), 0.84(\mathrm{~d}, J=6.6 \mathrm{~Hz}, 3 \mathrm{H}), 0.80(\mathrm{~m}, 1 \mathrm{H}) ;{ }^{13} \mathrm{C} \mathrm{NMR}$ (75 MHz, $\mathrm{CDCl}_{3}$ ): $\square 153.1,150.8,128.1,125.6,125.1,84.9,76.2,72.8,50.6,41.5,40.0$, 34.4, 31.4, 27.0, 26.8, 26.3, 21.7, 3.8; IR $\left(\mathrm{CH}_{2} \mathrm{Cl}_{2}\right.$ cast, $\left.\mathrm{cm}^{-1}\right): 2954,2254,1702,1600,1258$, 751, 700; HRMS (EI, $m / z$ ) Calcd for $\mathrm{C}_{20} \mathrm{H}_{26} \mathrm{O}_{2}, 298.19327$, found 298.19264; Anal. Calcd for $\mathrm{C}_{20} \mathrm{H}_{26} \mathrm{O}_{2}$ : C, 80.48; $\mathrm{H}, 8.80$. Found: $\mathrm{C}, 80.32 ; \mathrm{H}, 8.92$.

\subsubsection{2-Butynoic acid, 8-q-naphthylmenthol ester 10f}

This compound was prepared according to the procedure described in Section

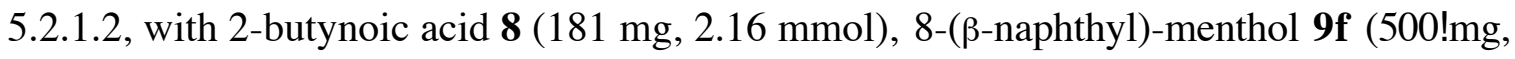
$1.78 \mathrm{mmol}$ ), DCC (429 mg, $2.08 \mathrm{mmol}$ ) and DMAP (21 mg, $0.17 \mathrm{mmol}$ ) except the mixture was left to stir at room temperature for $60 \mathrm{~h}$. Work up gave a dark orange slush $(767 \mathrm{mg})$. Flash chromatography $\left(10 \% \mathrm{Et}_{2} \mathrm{O} / \mathrm{Hexanes}, 28 \mathrm{~g}\right.$ silica, pre-absorption) gave the product $(496 \mathrm{mg})$. The product was further purified by Kugelrohr distillation $\left(0.1\right.$ torr, $250{ }^{\circ} \mathrm{C}$ ) to give an white crystalline solid (466 mg, $1.34 \mathrm{mmol}, 75 \%$ ).

mp 149-150 ${ }^{\circ} \mathrm{C}$; [0] ${ }_{\mathrm{D}}^{26}$ (-) 55.2 (c 1.56, $\mathrm{CHCl}_{3}$ ); ${ }^{1} \mathrm{H}$ NMR (500 MHz, $\left.\mathrm{CDCl}_{3}\right): \square 7.79$ (m, $3 \mathrm{H}), 7.63(\mathrm{~d}, J=1.7 \mathrm{~Hz}, 1 \mathrm{H}), 7.53(\mathrm{dd}, J=8.7 \mathrm{~Hz}, 2.0 \mathrm{~Hz}, 1 \mathrm{H}), 7.42(\mathrm{dt}, J=6.9 \mathrm{~Hz}$, $1.2 \mathrm{~Hz}, 1 \mathrm{H}), 7.36(\mathrm{dt}, J=6.9 \mathrm{~Hz}, 1.2 \mathrm{~Hz}, 1 \mathrm{H}), 4.92(\mathrm{dt}, J=10.7 \mathrm{~Hz}, 4.4 \mathrm{~Hz}, 1 \mathrm{H}), 2.15(\mathrm{~m}$, $1 \mathrm{H}), 1.86(\mathrm{~m}, 1 \mathrm{H}), 1.75(\mathrm{qd}, J=13.6 \mathrm{~Hz}, 3.5 \mathrm{~Hz}, 1 \mathrm{H}), 1.64(\mathrm{~m}, 1 \mathrm{H}), 1.46(\mathrm{~m}, 1 \mathrm{H}), 1.44(\mathrm{~s}$, 
$3 \mathrm{H}), 1.41(\mathrm{~s}, 3 \mathrm{H}), 1.31(\mathrm{~s}, 3 \mathrm{H}), 1.14(\mathrm{qd}, J=13.0 \mathrm{~Hz}, 3.5 \mathrm{~Hz}, 1 \mathrm{H}), 1.03(\mathrm{q}, J=12.2 \mathrm{~Hz}$, $1 \mathrm{H}), 0.90(\mathrm{qd}, J=13.0 \mathrm{~Hz}, 3.4 \mathrm{~Hz}, 1 \mathrm{H}), 0.86(\mathrm{~d}, J=6.4 \mathrm{~Hz}, 3 \mathrm{H}) ;{ }^{13} \mathrm{C}$ NMR $(125 \mathrm{MHz}$, $\left.\mathrm{CDCl}_{3}\right)$ : $\square 152.8,148.7,133.7,131.6,128.1,127.4,127.1,125.5,124.9,124.8,122.7,84.9$, 75.7, 72.2, 50.0, 41.5, 39.9, 34.5, 31.4, 28.0, 26.6, 24.6, 21.8, 3.2; IR $\left(\mathrm{CH}_{2} \mathrm{Cl}_{2}\right.$ cast, $\left.\mathrm{cm}^{-1}\right)$ : 3025, 2954, 2244, 1701, 1600, 1260, 854, 817, 749; HRMS (EI, $m / z$ ) Calcd for $\mathrm{C}_{24} \mathrm{H}_{28} \mathrm{O}_{2}$, 348.20892, found 348.20842; Anal. Calcd for $\mathrm{C}_{24} \mathrm{H}_{28} \mathrm{O}_{2}$ : C, 82.70; H, 8.11. Found: $\mathrm{C}, 82.52 ; \mathrm{H}, 8.19$.

\subsubsection{2-Butynoic acid, 8-(4-methoxyphenyl)menthol ester 10g}

This compound was prepared according to the procedure described in Section 5.2.1.2, with 2-butynoic acid 8 ( $0.385 \mathrm{~g}, 4.58 \mathrm{mmol}), 8$-(4-methoxyphenyl)-menthol $\mathbf{9 g}$ (1.11 g, $4.23 \mathrm{mmol})$, DCC (0.96 g, $4.67 \mathrm{mmol})$ and DMAP (15 mg, $0.12 \mathrm{mmol})$. Work up gave a dark orange slush. Flash chromatography $\left(5 \% \mathrm{Et}_{2} \mathrm{O} /\right.$ Hexanes, $55 \mathrm{~g}$ silica, pre-absorption) gave the solid product ( $896 \mathrm{mg}, 2.73 \mathrm{mmol}, 65 \%$ ).

mp 89-92 ${ }^{\circ} \mathrm{C}$; $[\mathrm{\square}]^{26}{ }_{\mathrm{D}}(+) 4.36\left(\mathrm{c} 2.89, \mathrm{CHCl}_{3}\right)$; IR $\left(\mathrm{CH}_{2} \mathrm{Cl}_{2}\right.$ cast, $\left.\mathrm{cm}^{-1}\right): 2954,2243,1701$, 1610, 1258, 1066, 830; HRMS (EI, $m / z$ ) Calcd for $\mathrm{C}_{21} \mathrm{H}_{28} \mathrm{O}_{3}, 328.20386$, found 328.20444; Anal. Calcd for $\mathrm{C}_{21} \mathrm{H}_{28} \mathrm{O}_{3}$ : C, 76.78; H, 8.61. Found: C, 76.46; H, 8.75.

\subsubsection{2-Butynoic acid, 8-(4-phenylphenyl)menthol ester 10h}

This compound was prepared according to the procedure described in Section 5.2.1.2, with 2-butynoic acid 8 (149 $\mathrm{mg}, 1.77 \mathrm{mmol}), 8$-(4-phenylphenyl)-menthol $\mathbf{9 h}$ (0.49 g, $1.6 \mathrm{mmol})$, DCC (0.36 g, $1.7 \mathrm{mmol})$ and DMAP (38 mg, $0.32 \mathrm{mmol})$ except that the mixture was left to stir at room temperature overnight. Work up gave a dark-orange slush $(0.77 \mathrm{~g})$. Flash chromatography $\left(5 \% \mathrm{Et}_{2} \mathrm{O} /\right.$ Hexanes, $100 \mathrm{~g}$ silica, pre-absorption) gave the product $(0.24 \mathrm{~g}, 0.64 \mathrm{mmol}, 35 \%)$, which was further purified by Kugelrohr distillation $\left(0.1\right.$ torr, $\left.250{ }^{\circ} \mathrm{C}\right)$ to give the product as a solid (146 $\left.\mathrm{mg}, 0.389 \mathrm{mmol}, 24 \%\right)$. mp 148-149 ${ }^{\circ} \mathrm{C}$; [c] $]_{\mathrm{D}}^{26}$ (+) 41.4 (c 2.34, $\mathrm{CHCl}_{3}$ ); ${ }^{1} \mathrm{H}$ NMR (500 MHz, $\mathrm{CDCl}_{3}$ ): 77.60 (m, $2 \mathrm{H}), 7.54(\mathrm{AB}, J=8.2 \mathrm{~Hz}, 2 \mathrm{H}), 7.39(\mathrm{~m}, 2 \mathrm{H}), 7.35(\mathrm{AB}, J=8.4 \mathrm{~Hz}, 2 \mathrm{H}), 7.28(\mathrm{~m}, 1 \mathrm{H})$, $4.89(\mathrm{dt}, J=10.8 \mathrm{~Hz}, 4.6 \mathrm{~Hz}, 1 \mathrm{H}), 2.02(\mathrm{~m}, 1 \mathrm{H}), 1.88(\mathrm{~m}, 1 \mathrm{H}), 1.70(\mathrm{~s}, 3 \mathrm{H}), 1.68(\mathrm{~m}, 1 \mathrm{H})$, $1.60(\mathrm{~m}, 1 \mathrm{H}), 1.43(\mathrm{~m}, 1 \mathrm{H}), 1.37(\mathrm{~s}, 3 \mathrm{H}), 1.28(\mathrm{~s}, 3 \mathrm{H}), 1.05(\mathrm{~m}, 2 \mathrm{H}), 0.86(\mathrm{~d}, J=6.4 \mathrm{~Hz}$, $3 \mathrm{H}), 0.84(\mathrm{~m}, 1 \mathrm{H}) ;{ }^{13} \mathrm{C}$ NMR $\left(125 \mathrm{MHz}, \mathrm{CDCl}_{3}\right)$ : $\square$ 152.9, 150.0, 140.8, 137.7, 128.6, 126.9, 126.7, 126.5, 125.9, 84.8, 75.8, 72.6, 50.6, 41.6, 39.8, 34.5, 31.4, 27.4, 26.8, 25.8, 21.8, 3.7; IR $\left(\mathrm{CH}_{2} \mathrm{Cl}_{2}\right.$ cast, $\left.\mathrm{cm}^{-1}\right): 3028,2955,2243,1701,1599,1259,837,767,698$; 
HRMS (EI, $m / z$ ) Calcd for $\mathrm{C}_{26} \mathrm{H}_{30} \mathrm{O}_{2}, 374.22458$, found 374.22467; Anal. Calcd for $\mathrm{C}_{26} \mathrm{H}_{30} \mathrm{O}_{2}$ : C, 83.37; H, 8.09. Found: C, 82.36; H, 8.40.

\subsubsection{2-Butynoic acid, 8-(3,5-dimethylphenyl)menthol ester 10i}

This compound was prepared according to the procedure described in Section 5.2.1.2, with 2-butynoic acid 8 (186 mg, $2.22 \mathrm{mmol}), 8$-(3,5-dimethylphenyl)-menthol 9i (462 mg, $1.77 \mathrm{mmol}$ ), DCC (0.47 g, $2.3 \mathrm{mmol}$ ) and DMAP (95 mg, $0.78 \mathrm{mmol}$ ) except the reaction was left to stir at room temperature for $36 \mathrm{~h}$. Work up gave a dark orange slush (770 mg). Flash chromatography ( $5 \% \mathrm{Et}_{2} \mathrm{O} /$ Hexanes, $43 \mathrm{~g}$ silica, pre-absorption) followed by Kugelrohr distillation $\left(0.1\right.$ torr, $\left.250^{\circ} \mathrm{C}\right)$ gave the product as a colourless oil $(251 \mathrm{mg}$, $0.768 \mathrm{mmol}, 43 \%)$.

$[\square]^{26}{ }_{\mathrm{D}}(+) 0.12\left(c\right.$ 1.65, $\left.\mathrm{CHCl}_{3}\right) ;{ }^{1} \mathrm{H}$ NMR $\left(500 \mathrm{MHz}, \mathrm{CDCl}_{3}\right): \square 6.86(\mathrm{~s}, 2 \mathrm{H}), 6.77(\mathrm{~s}, 1 \mathrm{H})$, $4.82(\mathrm{dt}, J=10.7 \mathrm{~Hz}, 4.4 \mathrm{~Hz}, 1 \mathrm{H}), 2.28(\mathrm{~s}, 6 \mathrm{H}), 1.91(\mathrm{~s}, 3 \mathrm{H}), 1.90(\mathrm{~m}, 2 \mathrm{H}), 1.55(\mathrm{~m}, 2 \mathrm{H})$, $1.41(\mathrm{~m}, 1 \mathrm{H}), 1.30(\mathrm{~s}, 3 \mathrm{H}), 1.23(\mathrm{~s}, 3 \mathrm{H}), 1.00(\mathrm{~m}, 2 \mathrm{H}), 0.84(\mathrm{~d}, J=6.6 \mathrm{~Hz}, 3 \mathrm{H}), 0.79(\mathrm{~m}$, $1 \mathrm{H})$; IR $\left(\mathrm{CH}_{2} \mathrm{Cl}_{2}\right.$ cast, $\left.\mathrm{cm}^{-1}\right): 2953,2243,1703,1599,1388,1370,1258,846,710$; HRMS (EI, $m / z$ ) Calcd for $\mathrm{C}_{22} \mathrm{H}_{30} \mathrm{O}_{2}, 326.22458$, found 326.22343; Anal. Calcd for $\mathrm{C}_{22} \mathrm{H}_{30} \mathrm{O}_{2}$ : C, 80.92; H, 9.28. Found: C, 80.61; H, 9.17.

\subsubsection{Chiral allylboronates 11}

\subsubsection{2-[(4,4,5,5-tetramethyl-1,3,2-dioxaborolan-2-yl)methyl]-3-methylbut-2-enoic acid, $(R)$-pantolactone ester 11a}

A slurry of $\mathrm{CuBr} \cdot \mathrm{SMe}_{2}(212 \mathrm{mg}, 1.03 \mathrm{mmol})$ in $\mathrm{THF}(3 \mathrm{~mL})$ at $0{ }^{\circ} \mathrm{C}$ under $\mathrm{Ar}$ was treated with $\mathrm{MeLi}(1.18 \mathrm{M}$ in ether, $1.8 \mathrm{~mL}, 2.1 \mathrm{mmol})$. Once a colourless solution formed ( $10 \mathrm{~min}$ ) the mixture was chilled to $-78{ }^{\circ} \mathrm{C}$ and treated via canula with a $-78{ }^{\circ} \mathrm{C}$ solution of butynoate $10 \mathrm{a}(206 \mathrm{mg}, 1.05 \mathrm{mmol})$ in THF $(0.5 \mathrm{~mL}+0.5 \mathrm{~mL}$ canula rinse) After $1 \mathrm{~h}$, the mixture was treated with HMPA $(1.8 \mathrm{~mL})$ and pinacol iodomethaneboronate $5 \mathbf{a}(917 \mathrm{mg}$, $3.42 \mathrm{mmol}$ ), stirred for $5 \mathrm{~min}$ and then placed in a $0{ }^{\circ} \mathrm{C}$ bath for $2 \mathrm{~h}$. ). After 5 minutes the mixture was brought to room temperature, left to stir for $2 \mathrm{~h}$, and then reaction was quenched with $\mathrm{NH}_{4} \mathrm{Cl}_{\text {(aq) }}(10 \mathrm{~mL})$. The layers were separated and the aqueous layer was extracted with ether $(4 \times 10 \mathrm{~mL})$. The combined organic layers were washed with water $(6 \times 10 \mathrm{~mL})$ and brine $(10 \mathrm{~mL})$, dried $\left(\mathrm{Na}_{2} \mathrm{SO}_{4}\right)$, and concentrated, yielding the crude allylboronate as a yellow oil (390 $\mathrm{mg},>100 \%)$.

${ }^{1} \mathrm{H}$ NMR (300 MHz, $\mathrm{CDCl}_{3}$ ): $\square 5.43$ (s, 1H), 4.02 (m, 2H), 2.11 (s, 3H), 1.93 (s, 2H), 1.82 
(s, 3H), 1.30-1.12 (m, 18H + impurities); ${ }^{13} \mathrm{C}$ NMR (50 MHz, $\left.\mathrm{CDCl}_{3}\right): \square$ 172.5, 167.3, $145.8,122.2,83.1,76.0,74.6,40.1,24.6,24.2,23.3,22.8,19.9 ;{ }^{11} \mathrm{~B}$ NMR (64 MHz, $\left.\mathrm{CDCl}_{3}\right)$ : $\mathrm{C} 32.7$; IR $\left(\mathrm{CH}_{2} \mathrm{Cl}_{2}\right.$ cast, $\left.\mathrm{cm}^{-1}\right): 2977,1792,1721,1628,1146$; HRMS (EI, $\mathrm{m} / z$ ) Calcd for $\mathrm{C}_{18} \mathrm{H}_{29}{ }^{11} \mathrm{BO}_{6}, 352.20572$, found 352.20556.

\subsubsection{2-[(4,4,5,5-tetramethyl-1,3,2-dioxaborolan-2-yl)methyl]-3-methylbut-2-enoic acid, (-)-borneol ester 11b}

This compound was prepared according to the procedure described in Section 5.2.2.1, with (-)-borneol butynoate $10 \mathrm{~b}(232 \mathrm{mg}, 1.05 \mathrm{mmol}), \mathrm{CuBr} \cdot \mathrm{SMe}_{2}(217 \mathrm{mg}$, $1.06 \mathrm{mmol}), \mathrm{MeLi}\left(1.29 \mathrm{M}\right.$ in $\left.\mathrm{Et}_{2} \mathrm{O}, 1.6 \mathrm{~mL}, 2.1 \mathrm{mmol}\right)$, and iodomethaneboronate $5 \mathbf{a}$ (842 mg, $3.14 \mathrm{mmol}$ ). Usual work-up gave the crude allylboronate as a yellow oil (453 mg, $>100 \%)$.

${ }^{1} \mathrm{H}$ NMR (300 MHz, CDCl $)$ : $\square 4.88(\mathrm{~m}, 1 \mathrm{H}), 2.36(\mathrm{~m}, 1 \mathrm{H}), 2.04(\mathrm{~s}, 3 \mathrm{H}), 1.96(\mathrm{~m}, 1 \mathrm{H})$, 1.89 (br s, 2H), $1.76(\mathrm{~s}, 3 \mathrm{H}), 1.72(\mathrm{~m}, 1 \mathrm{H}), 1.66(\mathrm{~m}, 1 \mathrm{H}), 1.24(\mathrm{~m}, 2 \mathrm{H}), 1.20(\mathrm{~s}, 12 \mathrm{H}), 1.01$ (m, 1H), 0.90 (s, 3H), 0.85 (s, 3H), $0.84(\mathrm{~s}, 3 \mathrm{H}) ;{ }^{13} \mathrm{C} \mathrm{NMR}\left(50 \mathrm{MHz}, \mathrm{CDCl}_{3}\right)$ : $\mathrm{N}$ 169.7, 141.9, 123.9, 83.0, 79.7, 48.6, 47.6, 44.8, 36.8, 28.0, 27.4, 24.7, 24.4, 23.0, 19.6, 18.8, 13.5;

${ }^{11} \mathrm{~B}$ NMR (64 MHz, $\left.\mathrm{CDCl}_{3}\right)$ : $\mathrm{q} 33.8$; IR $\left(\mathrm{CH}_{2} \mathrm{Cl}_{2}\right.$ cast $\left.\mathrm{cm}^{-1}\right)$ : 2978, 1708, 1632, 1371, 1112; HRMS (EI, $m / z$ ) Calcd for $\mathrm{C}_{22} \mathrm{H}_{37}{ }^{1}{ }^{1} \mathrm{BO}_{4}, 376.27850$, found 376.27917.

\subsubsection{2-[(4,4,5,5-tetramethyl-1,3,2-dioxaborolan-2-yl)methyl]-3-methylbut-2-enoic acid, $(R)$-1-phenylethanol ester 11c}

This compound was prepared according to the procedure described in Section 5.2.2.1, with (R)-1-phenylethyl butynoate 10c (621 mg, $3.30 \mathrm{mmol}), \mathrm{CuBr} \cdot \mathrm{SMe}_{2}(6.91 \mathrm{mg}$, $3.36 \mathrm{mmol}), \mathrm{MeLi}\left(1.37 \mathrm{M}\right.$ in $\left.\mathrm{Et}_{2} \mathrm{O}, 4.8 \mathrm{~mL}, 6.5 \mathrm{mmol}\right)$, and iodomethaneboronate $5 \mathrm{a}$ $(1.9 \mathrm{~mL}$, density $=1.4 \mathrm{~g} / \mathrm{mL}, 9.9 \mathrm{mmol})$. Usual work-up gave the crude allylboronate as a yellow oil $(1.12 \mathrm{~g}, 98 \%)$.

$\left.{ }^{1} \mathrm{H} \mathrm{NMR} \mathrm{(200} \mathrm{MHz,} \mathrm{CDCl}\right)$ : $77.30(\mathrm{~m}, 5 \mathrm{H}), 5.94$ (q, $\left.J=7 \mathrm{~Hz}, 1 \mathrm{H}\right), 2.06(\mathrm{~s}, 3 \mathrm{H}), 1.92$ (br s, 2H), $1.80(\mathrm{~s}, 3 \mathrm{H}), 1.57(\mathrm{~d}, J=7 \mathrm{~Hz}, 3 \mathrm{H}), 1.20(\mathrm{~s}, 12 \mathrm{H}) ;{ }^{13} \mathrm{C}$ NMR $\left(50 \mathrm{MHz}, \mathrm{CDCl}_{3}\right)$ : 口 168.2, 142.7, 142.2, 128.2, 127.4, 126.0, 123.6, 83.0, 71.8, 24.3, 23.1, 23.0, 22.5;

${ }^{11} \mathrm{~B}$ NMR (64 MHz, $\left.\mathrm{CDCl}_{3}\right)$ : $\square 31.7$; IR $\left(\mathrm{CH}_{2} \mathrm{Cl}_{2}\right.$ cast, $\left.\mathrm{cm}^{-1}\right): 2978,1711,1634,1347,1146$, 760, 699; HRMS (EI, $m / z$ ) Calcd for $\mathrm{C}_{20} \mathrm{H}_{29}{ }^{11} \mathrm{BO}_{4}, 344.21588$, found 344.21628 . 


\subsubsection{2-[(4,4,5,5-tetramethyl-1,3,2-dioxaborolan-2-yl)methyl]-3-methylbut-2-enoic acid,} (-)-menthol 11d

This compound was prepared according to the procedure described in Section 5.2.2.1, with (-)-menthol butynoate $10 d$ (198 $\mathrm{mg}, 0.890 \mathrm{mmol}), \mathrm{CuBr} \cdot \mathrm{SMe}_{2}$ (184 mg, $0.896 \mathrm{mmol}$ ), MeLi (1.29 $\mathrm{M}$ in $\mathrm{Et}_{2} \mathrm{O}, 1.4 \mathrm{~mL}, 1.8 \mathrm{mmol}$ ), and iodomethaneboronate $\mathbf{5 a}$ (810 mg, $3.02 \mathrm{mmol}$ ). Usual work-up gave the crude allylboronate as a yellow oil (316 $\mathrm{mg}$, $94 \%)$.

${ }^{1} \mathrm{H}$ NMR (300 MHz, $\mathrm{CDCl}_{3}$ ): $\square 4.75$ (dt, $\left.J=7.0 \mathrm{~Hz}, 4.5 \mathrm{~Hz}, 1 \mathrm{H}\right), 2.1-1.9$ (m, 2H), 2.00 (s, 3H), 1.84 (br s, 2H), 1.75 (s, 3H), 1.7-1.3 (m, 4H), 1.19 (s, 12H), 1.1-0.8 (m, 3H), 0.86 (m, $6 \mathrm{H}), 0.73(\mathrm{~d}, J=7.0 \mathrm{~Hz}, 3 \mathrm{H}) ;{ }^{13} \mathrm{C} \mathrm{NMR}\left(50 \mathrm{MHz}, \mathrm{CDCl}_{3}\right)$ : $\square$ 169.0, 141.3, 124.1, 83.0, 73.7, 47.1, 41.0, 34.3, 31.4, 25.6, 24.5, 23.0, 21.8, 20.7, 15.8; ${ }^{11} \mathrm{~B}$ NMR (64 MHz, $\left.\mathrm{CDCl}_{3}\right)$ : प31.6; IR $\left(\mathrm{CH}_{2} \mathrm{Cl}_{2}\right.$ cast, $\left.\mathrm{cm}^{-1}\right)$ : 2956, 1708, 1643, 1347, 1108; HRMS (EI, $\left.m / z\right)$ Calcd for $\mathrm{C}_{22} \mathrm{H}_{39}{ }^{11} \mathrm{BO}_{4}, 378.29413$, found 378.29497.

\subsubsection{2-[(4,4,5,5-tetramethyl-1,3,2-dioxaborolan-2-yl)methyl]-3-methylbut-2-enoic acid, (-)-8-phenylmenthol ester $11 \mathrm{e}$}

This compound was prepared according to the procedure described in Section 5.2.2.1, with (-)-8-phenylmenthol butynoate 10 e $(311 \mathrm{mg}, 1.04 \mathrm{mmol}), \mathrm{CuBr} \cdot \mathrm{SMe}_{2}$ (212 mg, $1.04 \mathrm{mmol}), \mathrm{MeLi}\left(1.4 \mathrm{M}\right.$ in $\left.\mathrm{Et}_{2} \mathrm{O}, 1.5 \mathrm{~mL}, 2.1 \mathrm{mmol}\right)$, and iodomethaneboronate 5 a $(0.60 \mathrm{~mL}, 3.1 \mathrm{mmol})$. Usual work-up gave the crude allylboronate as a yellow oil (511 mg). Flash chromatography $\left(10 \% \mathrm{Et}_{2} \mathrm{O} /\right.$ Hexanes, $23 \mathrm{~g}$ silica, pre-absorption) gave the purified compound as a colourless oil (209 $\mathrm{mg}, 0.460 \mathrm{mmol}, 44 \%)$.

${ }^{1} \mathrm{H}$ NMR (400 MHz, $\left.\mathrm{CDCl}_{3}\right)$ : $77.24(\mathrm{~m}, 4 \mathrm{H}), 7.10(\mathrm{~m}, 1 \mathrm{H}), 4.88(\mathrm{dt}, J=10.7 \mathrm{~Hz}, 4.4 \mathrm{~Hz}$, $1 \mathrm{H}), 1.94(\mathrm{~m}, 2 \mathrm{H}), 1.93(\mathrm{~s}, 3 \mathrm{H}), 1.72(\mathrm{~m}, 5 \mathrm{H}), 1.55(\mathrm{~m}, 1 \mathrm{H}), 1.42(\mathrm{~m}, 2 \mathrm{H}), 1.32(\mathrm{~s}, 3 \mathrm{H})$, $1.25(\mathrm{~s}, 3 \mathrm{H}), 1.22(\mathrm{~s}, 12 \mathrm{H}), 0.98(\mathrm{~m}, 2 \mathrm{H}), 0.83(\mathrm{~d}, J=6.9 \mathrm{~Hz}, 3 \mathrm{H}), 0.78(\mathrm{~m}, 1 \mathrm{H}) ;{ }^{13} \mathrm{C} \mathrm{NMR}$ (125 MHz, $\mathrm{CDCl}_{3}$ ): $\square 168.2,151.1,142.2,127.7,125.6,124.9,124.1,83.0,74.2,51.0,42.2$, $40.2,34.8,31.4,28.2,27.2,25.5,25.0,24.9,23.5,23.2,21.9 ;{ }^{11} \mathrm{~B}$ NMR $\left(64 \mathrm{MHz}, \mathrm{CDCl}_{3}\right)$ : प 34.9; IR $\left(\mathrm{CH}_{2} \mathrm{Cl}_{2}\right.$ cast, $\left.\mathrm{cm}^{-1}\right): 3056,2952,1701,1631,1600,1347,1147,767,700$; HRMS (EI, $m / z$ ) Calcd for $\mathrm{C}_{28} \mathrm{H}_{43}{ }^{11} \mathrm{BO}_{4}, 454.32544$, found 454.32597 . 


\subsubsection{2-[(4,4,5,5-tetramethyl-1,3,2-dioxaborolan-2-yl)methyl]-3-methylbut-2-enoic acid, 8-पnaphthylmenthol ester 11 ff}

This compound was prepared according to the procedure described in Section 5.2.2.1, with 8---napthylmenthol butynoate $\mathbf{1 0 f}(1.54 \mathrm{~g}, 4.42 \mathrm{mmol}), \mathrm{CuBr} \cdot \mathrm{SMe}_{2}(917 \mathrm{mg}$, $4.46 \mathrm{mmol}), \mathrm{MeLi}\left(1.27 \mathrm{M}\right.$ in $\left.\mathrm{Et}_{2} \mathrm{O}, 7.0 \mathrm{~mL}, 8.9 \mathrm{mmol}\right)$, and iodomethaneboronate $5 \mathrm{a}$ (3.91 g, $14.6 \mathrm{mmol})$. Usual work-up gave the crude allylboronate as a yellow oil (2.82 g, $>100 \%)$. Flash chromatography ( $25 \% \mathrm{Et}_{2} \mathrm{O} /$ Hexanes) of a small aliquot gave an analytical sample as a white solid.

TLC (25\% Et 2 O/Hexanes, UV/ $\left.\mathrm{KMnO}_{4}\right)$ : 0.47; ${ }^{1} \mathrm{H}$ NMR (500 MHz, $\left.\mathrm{CDCl}_{3}\right): 07.72(\mathrm{~m}$, $3 \mathrm{H}), 7.57(\mathrm{~s}, 1 \mathrm{H}), 7.46(\mathrm{dd}, J=8.7 \mathrm{~Hz}, 1.8 \mathrm{~Hz}, 1 \mathrm{H}), 7.36(\mathrm{~m}, 2 \mathrm{H}), 4.92(\mathrm{dt}, J=10.7 \mathrm{~Hz}$, $4.3 \mathrm{~Hz}, 1 \mathrm{H}), 2.09(\mathrm{~m}, 1 \mathrm{H}), 1.94(\mathrm{~m}, 1 \mathrm{H}), 1.75(\mathrm{~s}, 3 \mathrm{H}), 1.58(\mathrm{~m}, 2 \mathrm{H}), 1.48(\mathrm{br} \mathrm{s}, 2 \mathrm{H}), 1.46(\mathrm{~s}$, $1 \mathrm{H}), 1.40(\mathrm{~s}, 3 \mathrm{H}), 1.38(\mathrm{~s}, 3 \mathrm{H}), 1.31(\mathrm{~s}, 3 \mathrm{H}), 1.19(\mathrm{~s}, 12 \mathrm{H}), 1.09(\mathrm{~m}, 1 \mathrm{H}), 0.98$ (q, $J=11.0 \mathrm{~Hz}, 1 \mathrm{H}), 0.84(\mathrm{~m}, 1 \mathrm{H}), 0.83(\mathrm{~d}, J=6.6 \mathrm{~Hz}, 3 \mathrm{H}) ;{ }^{13} \mathrm{C} \mathrm{NMR}\left(125 \mathrm{MHz}, \mathrm{CDCl}_{3}\right)$ : $\mathrm{Q} 168.1,149.0,142.6,133.3,131.4,127.9,127.2,127.1,125.4,125.0,124.8,123.7,123.0$, 83.0, 73.9, 50.5, 42.2, 40.2, 34.8, 31.4, 27.0, 26.6, 24.9, 24.8, 23.3, 23.1, 21.9; ${ }^{11} \mathrm{~B}$ NMR $\left(64 \mathrm{MHz}, \mathrm{CDCl}_{3}\right)$ : $\mathrm{Q} 32.9$; IR $\left(\mathrm{CH}_{2} \mathrm{Cl}_{2}\right.$ cast, $\left.\mathrm{cm}^{-1}\right): \quad 3066,2975,1686,1632,1601,1357$, 1145, 876, 819, 749; HRMS (EI, $m / z$ ) Calcd for $\mathrm{C}_{32} \mathrm{H}_{45}{ }^{11} \mathrm{BO}_{4}, 504.34109$, found 504.34234 .

\subsubsection{2-[(4,4,5,5-tetramethyl-1,3,2-dioxaborolan-2-yl)methyl]-3-methylbut-2-enoic acid, 8-(4-methoxyphenyl)menthol ester $11 \mathrm{~g}$}

This compound was prepared according to the procedure described in Section 5.2.2.1, with 8-(4-anisyl)menthol butynoate $10 \mathrm{~g}$ (821 mg, $2.50 \mathrm{mmol}), \mathrm{CuBr} \cdot \mathrm{SMe}_{2}$

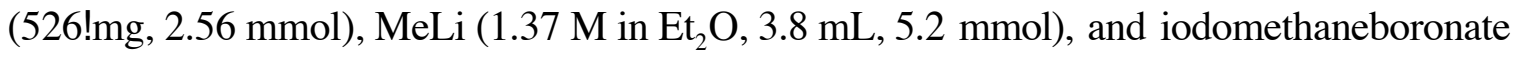
$\mathbf{5 a}(2.28 \mathrm{~g}, 8.51 \mathrm{mmol})$. Usual work-up gave the crude allylboronate as a yellow oil (1.68 g, $>100 \%)$.

${ }^{1} \mathrm{H}$ NMR $\left(500 \mathrm{MHz}, \mathrm{CDCl}_{3}\right): \square 7.15(\mathrm{AB}, J=8.9 \mathrm{~Hz}, 2 \mathrm{H}), 6.76(\mathrm{AB}, J=8.9 \mathrm{~Hz}, 2 \mathrm{H}), 4.85$ (dt, $J=10.7 \mathrm{~Hz}, 4.4 \mathrm{~Hz}, 1 \mathrm{H}), 3.76$ (s, 3H), 2.0-1.7 (m, 4H), 1.94 (s, 3H), 1.73 (s, 3H), 1.53 (m, 1H), $1.40(\mathrm{~m}, 2 \mathrm{H}), 1.28(\mathrm{~s}, 3 \mathrm{H}), 1.24(\mathrm{~s}, 3 \mathrm{H}), 1.19(\mathrm{~s}, 12 \mathrm{H}), 1.0-0.7(\mathrm{~m}, 3 \mathrm{H}), 0.81(\mathrm{~d}$, $J=6.0 \mathrm{~Hz}, 3 \mathrm{H}) ;{ }^{13} \mathrm{C}$ NMR $\left(125 \mathrm{MHz}, \mathrm{CDCl}_{3}\right)$ : $\square$ 168.3, 156.9, 143.2, 142.2, 126.6, 124.1, 113.0, 83.1, 74.3, 55.2, 51.1, 42.2, 39.6, 34.7, 31.4, 28.6, 27.2, 24.9, 24.8, 24.5, 23.3, 21.9; ${ }^{11} \mathrm{~B}$ NMR (64 MHz, $\left.\mathrm{CDCl}_{3}\right)$ : $\square 31.6$; IR $\left(\mathrm{CH}_{2} \mathrm{Cl}_{2}\right.$ cast, $\left.\mathrm{cm}^{-1}\right): 2975,1701,1611,1600,1346$, 1146, 830; HRMS (EI, $m / z$ ) Calcd for $\mathrm{C}_{29} \mathrm{H}_{45}{ }^{11} \mathrm{BO}_{5}, 484.33600$, found 484.33623 . 


\subsubsection{2-[(4,4,5,5-tetramethyl-1,3,2-dioxaborolan-2-yl)methyl]-3-methylbut-2-enoic acid, 8-(4-phenylphenyl)menthol ester $11 \mathrm{~h}$}

This compound was prepared according to the procedure described in Section 5.2.2.1, with 8-(4-phenylphenyl)menthol butynoate 10h (146 mg, $0.389 \mathrm{mmol}), \mathrm{CuBr} \cdot \mathrm{SMe}_{2}$ (80 mg, $0.39 \mathrm{mmol}$ ), MeLi (1.27 $\mathrm{M}$ in $\mathrm{Et}_{2} \mathrm{O}, 0.61 \mathrm{~mL}, 0.77 \mathrm{mmol}$ ), and iodomethaneboronate $\mathbf{5 a}(477 \mathrm{mg}, 1.78 \mathrm{mmol})$. Usual work-up gave the crude allylboronate as a yellow oil $(250 \mathrm{mg},>100 \%)$.

${ }^{1} \mathrm{H}$ NMR (500 MHz, $\mathrm{CDCl}_{3}$ ): $\mathrm{\square} 7.6-7.3$ (m, 9H), 4.80 (m, 1H), 2.1-1.9 (m, 2H), 1.94 (br s, $2 \mathrm{H}), 1.90(\mathrm{~s}, 3 \mathrm{H}), 1.7-1.4(\mathrm{~m}, 3 \mathrm{H}), 1.64$ (s, 3H), $1.38(\mathrm{~s}, 3 \mathrm{H}), 1.24(\mathrm{~s}, 3 \mathrm{H}), 1.20(\mathrm{~s}, 12 \mathrm{H})$, 1.1-0.8 (s, 6H); ${ }^{11} \mathrm{~B}$ NMR $\left(64 \mathrm{MHz}, \mathrm{CDCl}_{3}\right)$ : $\square 31.6$; IR $\left(\mathrm{CH}_{2} \mathrm{Cl}_{2}\right.$ cast, $\left.\mathrm{cm}^{-1}\right)$ : 3027, 2962, 1702, 1599, 1343, 1144, 801, 767, 698; HRMS (EI, $m / z$ ) Calcd for $\mathrm{C}_{34} \mathrm{H}_{47}{ }^{11} \mathrm{BO}_{4}$, 530.35675 , found 530.35769 .

\subsubsection{2-[(4,4,5,5-tetramethyl-1,3,2-dioxaborolan-2-yl)methyl]-3-methylbut-2-enoic acid, 8-(3,5-dimethylphenyl)menthol ester 11i}

This compound was prepared according to the procedure described in Section 5.2.2.1, with 8-(3,5-dimethylphenyl)menthol butynoate $10 \mathbf{i}(221 \mathrm{mg}, 0.677 \mathrm{mmol})$, $\mathrm{CuBr} \cdot \mathrm{SMe}_{2}(143 \mathrm{mg}, 0.698 \mathrm{mmol})$, MeLi (1.27 $\mathrm{M}$ in $\left.\mathrm{Et}_{2} \mathrm{O}, 1.1 \mathrm{~mL}, 1.4 \mathrm{mmol}\right)$, and iodomethaneboronate $\mathbf{5 a}(514 \mathrm{mg}, 1.92 \mathrm{mmol})$. Usual work-up gave the crude allylboronate as a yellow oil (342 $\mathrm{mg},>100 \%)$.

${ }^{11} \mathrm{~B}$ NMR (64 MHz, $\left.\mathrm{CDCl}_{3}\right)$ : $\square 31.4$; IR $\left(\mathrm{CH}_{2} \mathrm{Cl}_{2}\right.$ cast, $\left.\mathrm{cm}^{-1}\right)$ : 2922, 1704, 1633, 1599, 1343, 1145, 845, 710; HRMS (EI, $m / z$ ) Calcd for $\mathrm{C}_{30} \mathrm{H}_{47}{ }^{11} \mathrm{BO}_{4}, 482.35675$, found 482.35614 .

\subsubsection{Enantioenriched lactones from reaction with chiral allylboronates 11}

\subsubsection{Allylboration with $(R)$-pantolactone derived 11a}

Allylboronate 11a (276 mg, $1.03 \mathrm{mmol}$ ) and 3-(tert-butyldiphenylsilyloxy)propanal $12(217 \mathrm{mg}, 0.695 \mathrm{mmol})$ were stirred together at room temperature for $8 \mathrm{~d}$. The mixture was then purified by flash chromatography (5\% EtOAc/Toluene, $25 \mathrm{~g}$ silica) to give the enantioenriched lactone 2c (190 mg, $0.466 \mathrm{mmol}, 67 \%)$. Enantiomeric excess (CHIRALPAK AD-RH, $50 \%$ isopropanol/water, $0.3 \mathrm{~mL} / \mathrm{min}$ ): $22 \%$ for the $(S)$-enantiomer. 


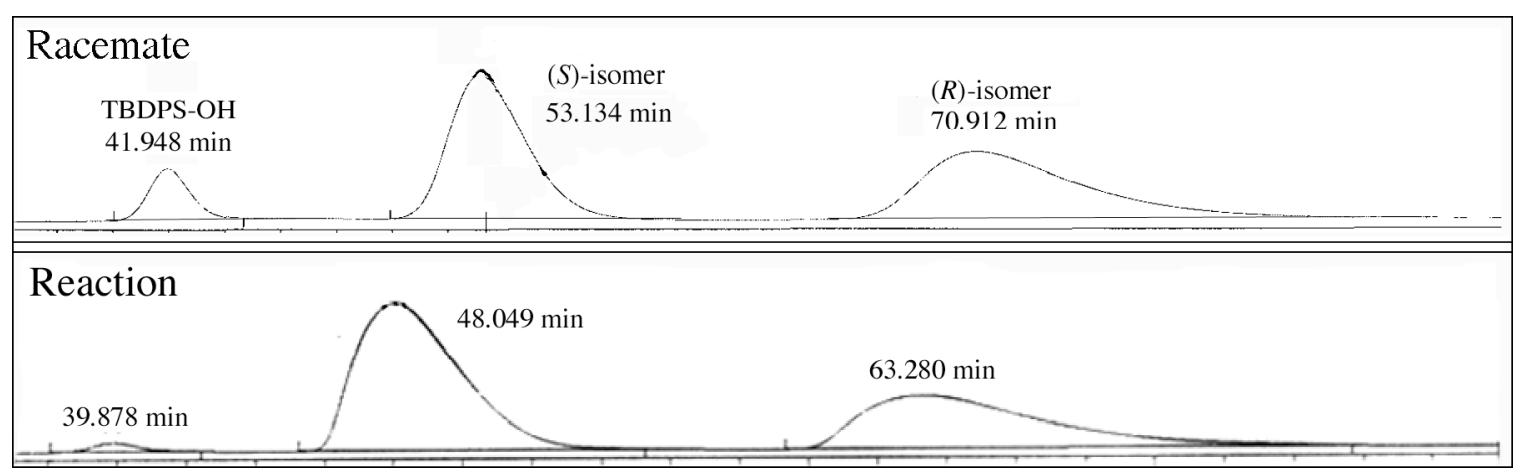

\subsubsection{Allylboration with (-)--borneol derived 11b}

Allylboronate 11b (416 mg, $1.13 \mathrm{mmol})$ and 3-(tert-butyldiphenylsilyloxy)propanal $12(261 \mathrm{mg}, 0.836 \mathrm{mmol})$ were stirred together at room temperature for $8 \mathrm{~d}$. The mixture was then purified by flash chromatography $\left(20 \% \mathrm{Et}_{2} \mathrm{O} / \mathrm{Hexanes}, 20 \mathrm{~g}\right.$ silica $)$ to give the enantioenriched lactone 2c (229 $\mathrm{mg}, 0.561 \mathrm{mmol}, 64 \%)$. Enantiomeric excess (CHIRALPAK AD-RH, $50 \%$ isopropanol/water, $0.3 \mathrm{~mL} / \mathrm{min}$ ): $6 \%$ for the $(R)$-enantiomer.

\begin{tabular}{|l|l} 
Racemate \\
TBDPS-OH \\
$41.948 \mathrm{~min}$
\end{tabular}

\subsubsection{Allylboration with $(R)-1$-phenylethanol derived 11c}

Allylboronate 11c (520 mg, $1.51 \mathrm{mmol}$ ) and 3-(tert-butyldiphenylsilyloxy)propanal $12(325 \mathrm{mg}, 1.04 \mathrm{mmol})$ were stirred together in toluene $(1 \mathrm{~mL})$ at room temperature for $26 \mathrm{~d}$. The mixture was then purified by flash chromatography (5\% EtOAc/Toluene, $25 \mathrm{~g}$ silica) to give the enantioenriched lactone $2 \mathrm{c}(0.30 \mathrm{~g}, 0.734 \mathrm{mmol}, 70 \%)$. Enantiomeric excess (CHIRALPAK AD-RH, 50\% isopropanol/water, $0.3 \mathrm{~mL} / \mathrm{min}$ ): $13 \%$ for the $(S)$-enantiomer. 


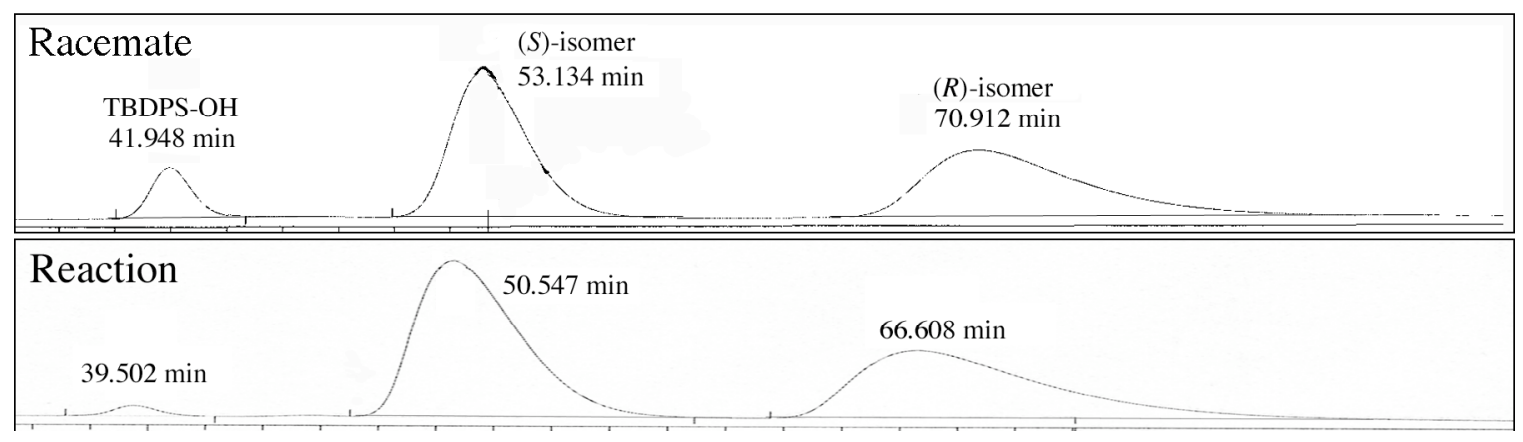

\subsubsection{Allylboration with (-)-menthol derived 11d}

Allylboronate 11d (270 mg, $0.71 \mathrm{mmol}$ ) and 3-(tert-butyldiphenylsilyloxy)propanal $12(149 \mathrm{mg}, 0.476 \mathrm{mmol})$ were stirred together at room temperature for $8 \mathrm{~d}$. The mixture was then purified by flash chromatography (5\% EtOAc/Toluene, $20 \mathrm{~g}$ silica) to give the enantioenriched lactone 2c (150 mg, $0.367 \mathrm{mmol}, 78 \%)$. Enantiomeric excess (CHIRALPAK AD-RH, 50\% isopropanol/water, $0.3 \mathrm{~mL} / \mathrm{min}$ ): $7 \%$ for the $(S)$-enantiomer.

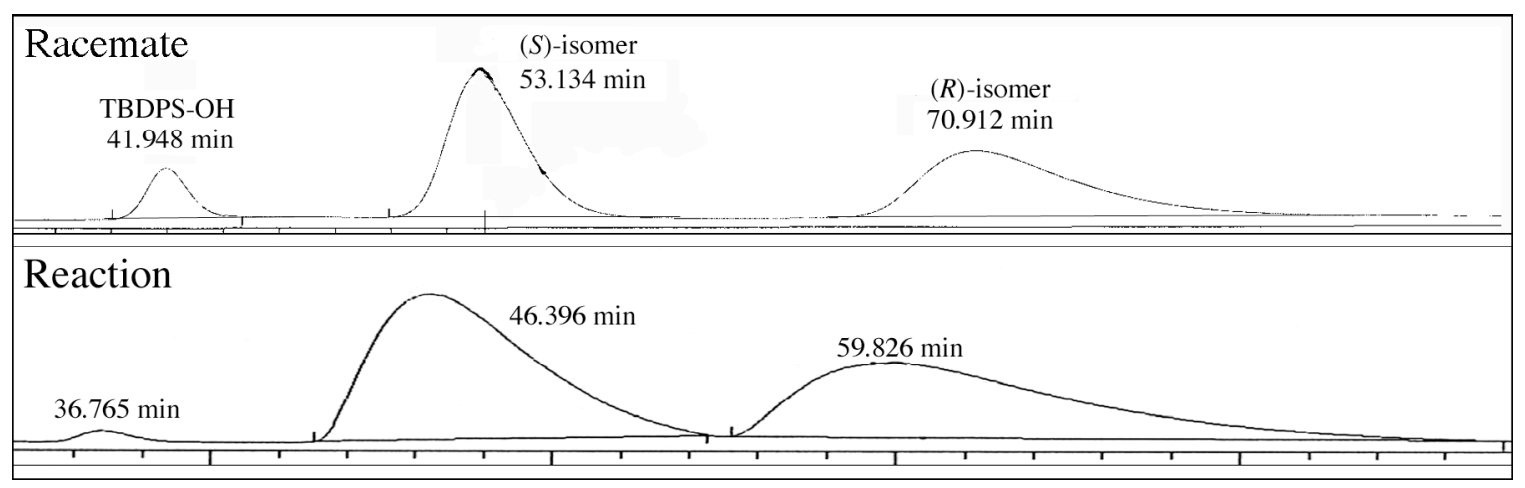

\subsubsection{Allylboration with (-)-8-phenylmenthol derived 11e}

Allylboronate $\quad \mathbf{1} \mathbf{e} \quad\left(\begin{array}{llll}372 & \mathrm{mg}, & 0.819 & \mathrm{mmol}\end{array}\right)$ and 3-(tert-butyldiphenylsilyloxy)propanal $12(168 \mathrm{mg}, 0.537 \mathrm{mmol})$ were stirred together in toluene $(1 \mathrm{~mL})$ at room temperature for $36 \mathrm{~d}$. A spatula tip of pTSA was then added and the mixture was left to stir for $30 \mathrm{~min}$. The reaction was then quenched with $\mathrm{NaHCO}_{3 \text { (aq) }}$ $(5 \mathrm{~mL})$ and extracted with $\mathrm{Et}_{2} \mathrm{O}(3 \times 5 \mathrm{~mL})$. The combined organic layers were washed with brine $(5 \mathrm{~mL})$, dried $\left(\mathrm{Na}_{2} \mathrm{SO}_{4}\right)$ and concentrated to give the crude lactone $2 \mathbf{c}$, which was then purified by flash chromatography to give the pure product (111 $\mathrm{mg}, 0.272 \mathrm{mmol}, 51 \%$ ). Enantiomeric excess (CHIRALPAK AD-RH, 50\% isopropanol/water, $0.3 \mathrm{~mL} / \mathrm{min}$ ): $80 \%$ for the $(S)$-enantiomer. 


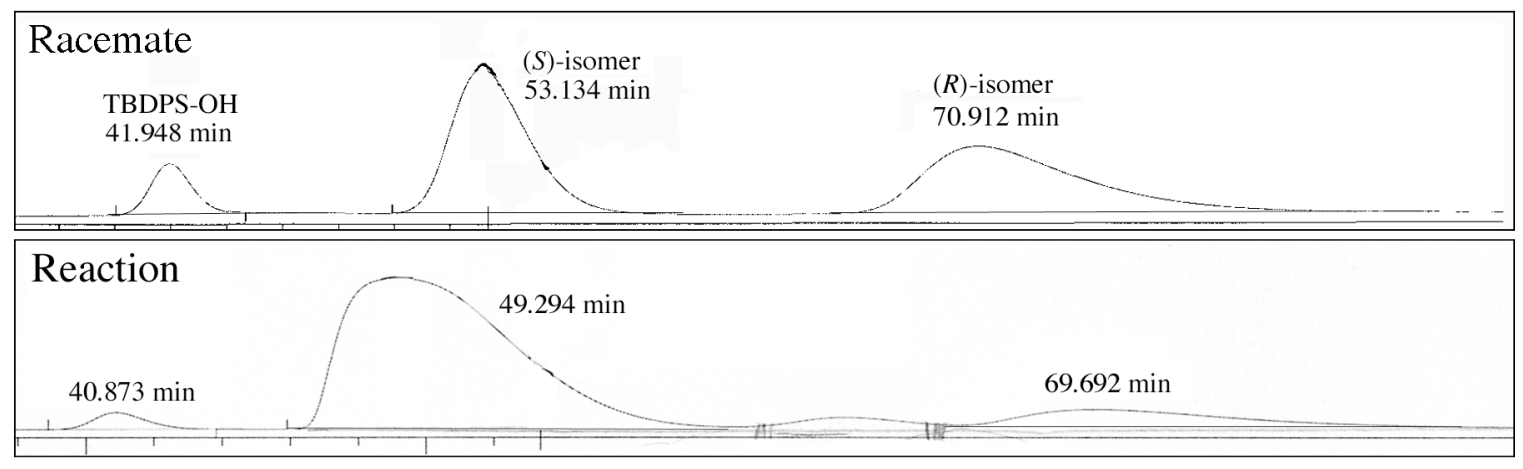

\subsubsection{Allylboration with $\square$-naphthylmenthol derived $11 \mathrm{f}$ in toluene}

Allylboronate 11f (565 mg, $1.12 \mathrm{mmol}$ ) and 3-(tert-butyldiphenylsilyloxy)propanal $12(243 \mathrm{mg}, 0.778 \mathrm{mmol})$ were stirred together in toluene $(1 \mathrm{~mL})$ at room temperature for $15 \mathrm{~d}$. A spatula tip of pTSA was then added and the mixture was left to stir for $30 \mathrm{~min}$. The reaction was then quenched with $\mathrm{NaHCO}_{3(\mathrm{aq})}(5 \mathrm{~mL})$ and extracted with $\mathrm{Et}_{2} \mathrm{O}$ $(3 \times 5 \mathrm{~mL})$. The combined organic layers were washed with brine $(5 \mathrm{~mL})$, dried $\left(\mathrm{Na}_{2} \mathrm{SO}_{4}\right)$ and concentrated to give the crude lactone $2 \mathbf{c}(674 \mathrm{mg})$, which was then purified by flash chromatography (10\% acetone/hexanes, $60 \mathrm{~g}$ silica) to give the compound still contaminated with the free arylmenthol (300 mg, 95\%). Enantiomeric excess (CHIRALPAK AD-RH, $50 \%$ isopropanol/water, $0.3 \mathrm{~mL} / \mathrm{min}$ ): $82 \%$ for the $(S)$-enantiomer.
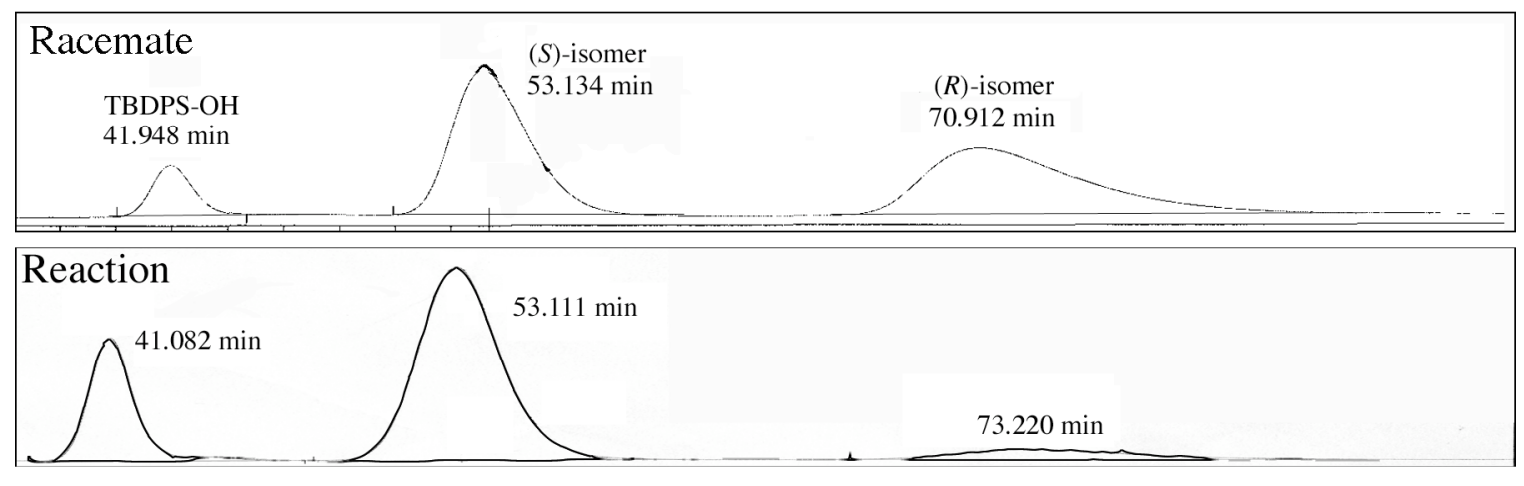

\subsubsection{Allylboration with 8---naphthylmenthol derived $11 \mathrm{f}$ in $\mathrm{CH}_{2} \mathrm{Cl}_{2}$}

Allylboronate $\mathbf{1 1 f}(456 \mathrm{mg}, 0.904 \mathrm{mmol})$ and 3-(tert-butyldiphenylsilyloxy)propanal $12(196 \mathrm{mg}, 0.628 \mathrm{mmol})$ were stirred together in $\mathrm{CH}_{2} \mathrm{Cl}_{2}(1 \mathrm{~mL})$ at room temperature for $14 \mathrm{~d}$. A spatula tip of pTSA was then added and 
the mixture was left to stir for $30 \mathrm{~min}$. The reaction was then quenched with $\mathrm{NaHCO}_{3(\mathrm{aq})}$ $(5 \mathrm{~mL})$ and extracted with $\mathrm{Et}_{2} \mathrm{O}(3 \times 5 \mathrm{~mL})$. The combined organic layers were washed with brine $(5 \mathrm{~mL})$, dried $\left(\mathrm{Na}_{2} \mathrm{SO}_{4}\right)$ and concentrated to give the crude lactone $2 \mathbf{c}(674 \mathrm{mg})$, which was then purified by flash chromatography ( $10 \%$ acetone/hexanes, $60 \mathrm{~g}$ silica) to give the compound still contaminated with the free arylmenthol $(0.22 \mathrm{~g}, 86 \%)$. Enantiomeric excess (CHIRALPAK AD-RH, 50\% isopropanol/water, $0.3 \mathrm{~mL} / \mathrm{min}$ ): $75 \%$ for the $(S)$-enantiomer.
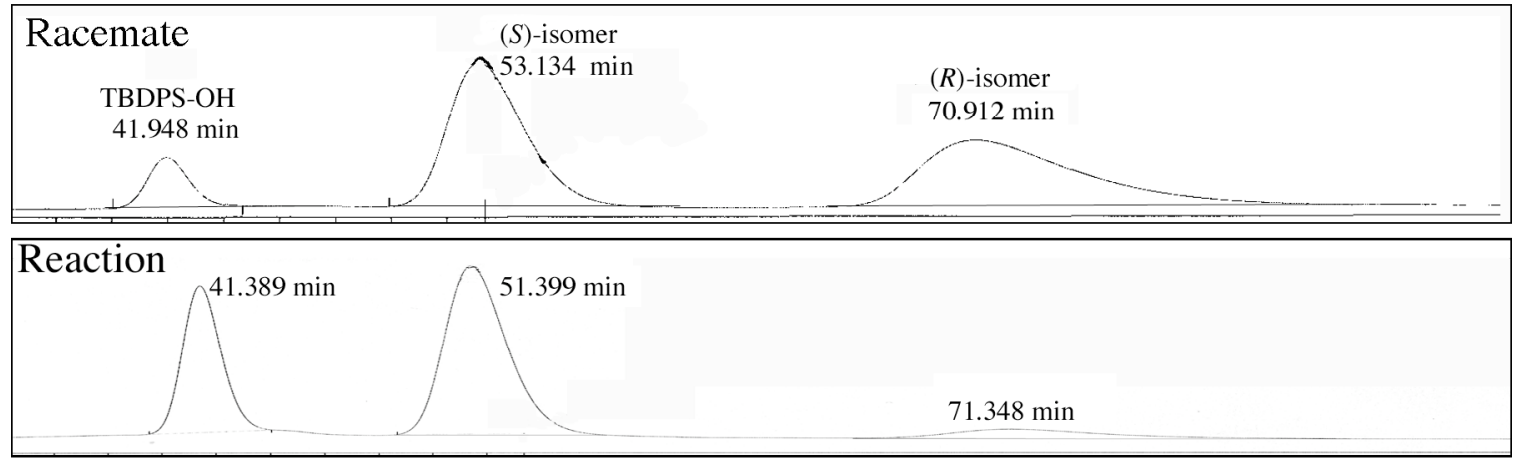

\subsubsection{Allylboration with 8-(4-methoxyphenyl)menthol derived 11g}

Allylboronate 11g (515 mg, $1.06 \mathrm{mmol}$ ) and 3-(tert-butyldiphenylsilyloxy)propanal $12(230 \mathrm{mg}, 0.737 \mathrm{mmol})$ were stirred together in toluene $(1 \mathrm{~mL})$ at room temperature for $14 \mathrm{~d}$. A spatula tip of pTSA was then added and the mixture was left to stir for $4 \mathrm{~h}$. The reaction was then quenched with $\mathrm{NaHCO}_{3 \text { (aq) }}(5 \mathrm{~mL})$ and extracted with $\mathrm{Et}_{2} \mathrm{O}(3 \times 5 \mathrm{~mL})$. The combined organic layers were washed with brine $(5 \mathrm{~mL})$, dried $\left(\mathrm{Na}_{2} \mathrm{SO}_{4}\right)$ and concentrated to give the crude lactone $2 \mathrm{c}(571 \mathrm{mg})$, which was then purified by flash chromatography (5\% EtOAc/toluene, $25 \mathrm{~g}$ silica) to give the pure compound (223 $\mathrm{mg}$, $0.547 \mathrm{mmol}, 74 \%$ ). Enantiomeric excess (CHIRALPAK AD-RH, 50\% isopropanol/water, $0.3 \mathrm{~mL} / \mathrm{min}): 56 \%$ for the $(S)$-enantiomer.

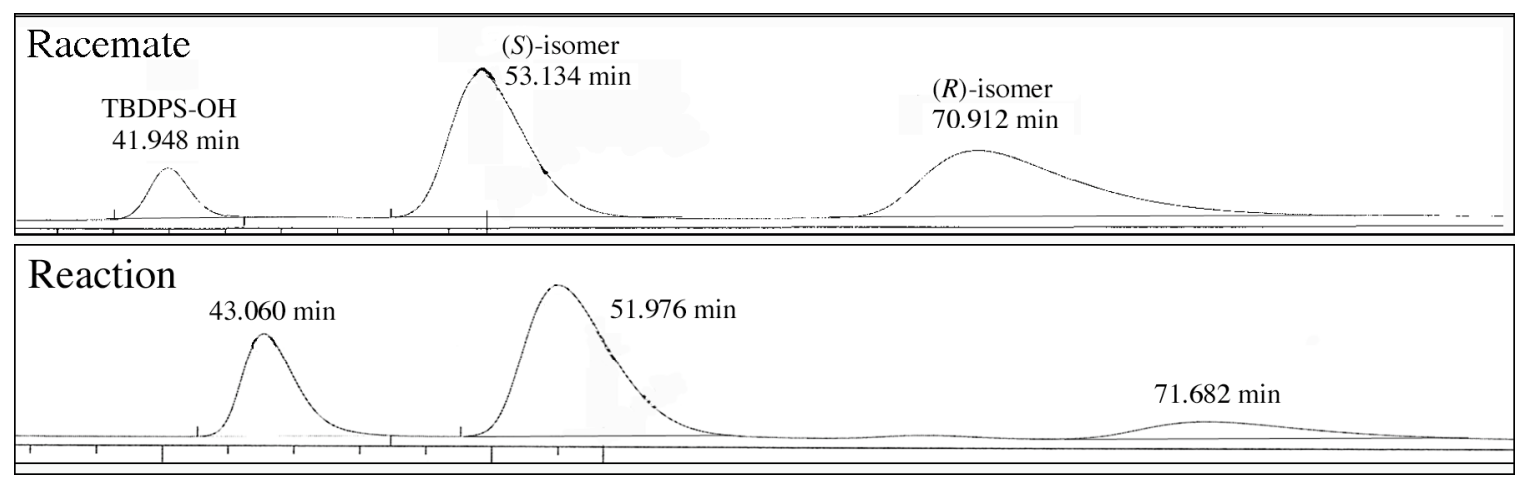




\subsubsection{Allylboration with 8-(4-phenylphenyl)menthol derived 11h}

Allylboronate $\quad \mathbf{1} \mathbf{1} \mathbf{h}(250 \quad \mathrm{mg}, \quad 0.472 \quad \mathrm{mmol})$ and 3-(tert-butyldiphenylsilyloxy)propanal $12(104 \mathrm{mg}, 0.333 \mathrm{mmol})$ were stirred together in toluene $(1 \mathrm{~mL})$ at room temperature for $24 \mathrm{~d}$. A spatula tip of pTSA was then added and the mixture was left to stir for $30 \mathrm{~min}$. The reaction was then quenched with $\mathrm{NaHCO}_{3(\mathrm{aq})}$ $(5 \mathrm{~mL})$ and extracted with $\mathrm{Et}_{2} \mathrm{O}(3 \times 5 \mathrm{~mL})$. The combined organic layers were washed with brine $(5 \mathrm{~mL})$, dried $\left(\mathrm{Na}_{2} \mathrm{SO}_{4}\right)$ and concentrated to give the crude lactone $\mathbf{2 c}$, which was then purified by flash chromatography $\left(40 \% \mathrm{CH}_{2} \mathrm{Cl}_{2} /\right.$ Hexanes, $35 \mathrm{~g}$ silica $)$ to give the pure compound (33 mg, $0.081 \mathrm{mmol}, 24 \%$ ). Enantiomeric excess (CHIRALPAK AD-RH, $50 \%$ isopropanol/water, $0.3 \mathrm{~mL} / \mathrm{min})$ : $66 \%$ for the $(S)$-enantiomer.

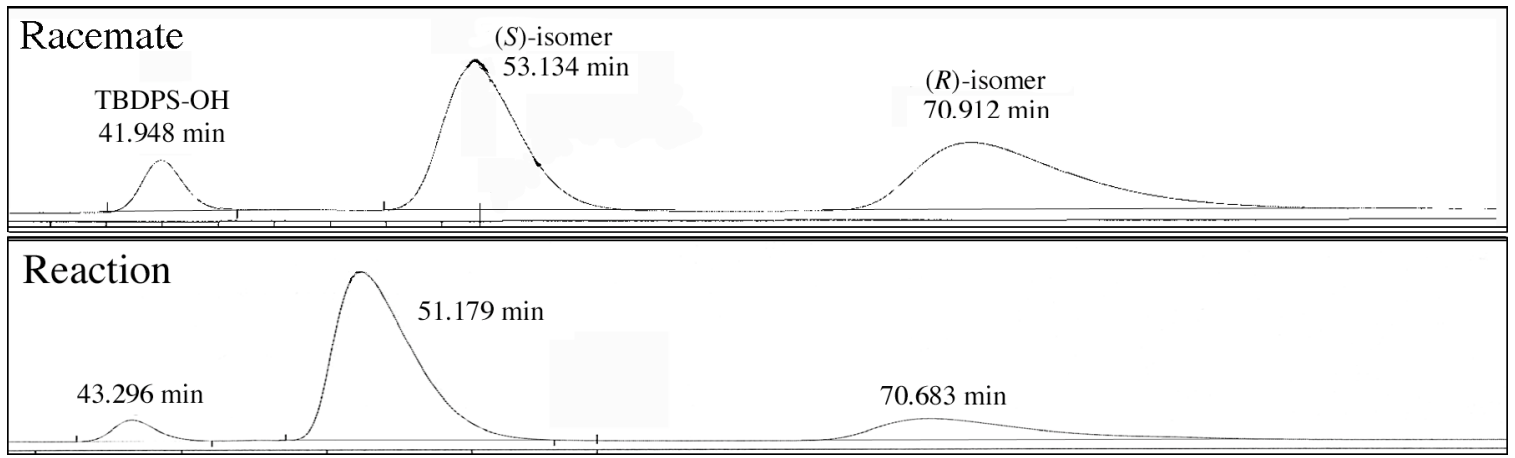

\subsubsection{Allylboration with 8-(3,5-dimethylphenyl)menthol derived 11i}

Allylboronate $11 \mathbf{i}(342 \mathrm{mg}, 0.708 \quad \mathrm{mmol})$ and 3-(tert-butyldiphenylsilyloxy)propanal $12(147 \mathrm{mg}, 0.470 \mathrm{mmol})$ were stirred together in toluene $(0.5 \mathrm{~mL})$ at room temperature for $15 \mathrm{~d}$. A spatula tip of pTSA was then added and the mixture was left to stir for $30 \mathrm{~min}$. The reaction was then quenched with $\mathrm{NaHCO}_{3(\mathrm{aq})}$ $(5 \mathrm{~mL})$ and extracted with $\mathrm{Et}_{2} \mathrm{O}(3 \times 5 \mathrm{~mL})$. The combined organic layers were washed with brine $(5 \mathrm{~mL})$, dried $\left(\mathrm{Na}_{2} \mathrm{SO}_{4}\right)$ and concentrated to give the crude lactone $2 \mathrm{c}(78 \mathrm{mg})$, which was then purified by flash chromatography (5\% EtOAc/toluene, $11 \mathrm{~g}$ silica) to give the compound still contaminated with the free arylmenthol (78 $\mathrm{mg}, 41 \%)$. A second chromatography step ( $40 \% \mathrm{CH}_{2} \mathrm{Cl}_{2} / \mathrm{Hexanes,} 5 \mathrm{~g}$ silica) gave the pure compound (11 $\mathrm{mg}$, $0.026 \mathrm{mmol}, 6 \%$ ). Enantiomeric excess (CHIRALPAK AD-RH, 50\% isopropanol/water, $0.3 \mathrm{~mL} / \mathrm{min}): 62 \%$ for the $(S)$-enantiomer. 


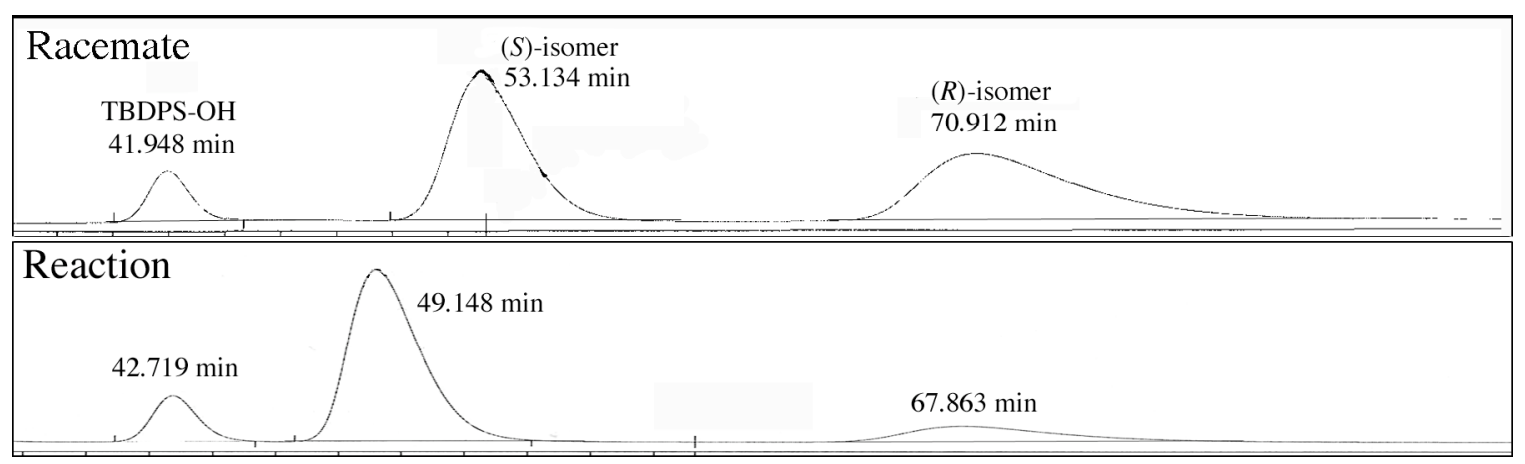

\subsection{Enantioselective allylborations - Dual auxiliary approach}

\subsection{1 (1R, 2R, 3R, 4S)-4-lodomethyl-1,10,10-trimethyl-2-phenyl-3,5-dioxa-4-bora- tricyclo[5.2.1.00,0]decane 15}

A solution of diisopropyl bromomethaneboronate 16a $(1.30 \mathrm{~g}, 5.84 \mathrm{mmol})$ in THF $(20 \mathrm{~mL})$ was treated with bornanediol (-)-14 (1.22 g, $4.96 \mathrm{mmol})$ and stirred under $\mathrm{Ar}$ overnight. In the morning the solvents were removed by distillation under Ar (bath temperature $110{ }^{\circ} \mathrm{C}$ ) to give the crude bromomethaneboronate as a brown oil. This oil was dissolved in acetone $(10 \mathrm{~mL})$, treated with $\mathrm{NaI}(1.58 \mathrm{~g}, 10.6 \mathrm{mmol})$ and refluxed for $60 \mathrm{~h}$. The mixture was then filtered and concentrated by rotor evaporator. The residue taken up in $\mathrm{CH}_{2} \mathrm{Cl}_{2}(50 \mathrm{~mL})$, filtered, and concentrated again to give the crude product $(2.08 \mathrm{~g})$. Kugelrohr distillation $\left(150{ }^{\circ} \mathrm{C}, 0.1\right.$ torr) removed a volatile impurity. Continued distillation at $250{ }^{\circ} \mathrm{C}$ gave the pure product as a slightly coloured oil $(1.57 \mathrm{~g}, 3.96 \mathrm{mmol}, 80 \%)$.

${ }^{1} \mathrm{H}$ NMR (500 MHz, $\left.\mathrm{CDCl}_{3}\right): \square 7.40(\mathrm{~m}, 2 \mathrm{H}), 7.32(\mathrm{~m}, 2 \mathrm{H}), 7.27(\mathrm{~m}, 1 \mathrm{H}), 4.79(\mathrm{~s}, 1 \mathrm{H})$, $2.16(\mathrm{~m}, 3 \mathrm{H}), 1.82(\mathrm{~m}, 1 \mathrm{H}), 1.24(\mathrm{~s}, 3 \mathrm{H}), 1.18(\mathrm{~m}, 2 \mathrm{H}), 1.03(\mathrm{~m}, 1 \mathrm{H}), 0.95(\mathrm{~s}, 3 \mathrm{H}), 0.93$ (s, $3 \mathrm{H}) ;{ }^{13} \mathrm{C} \mathrm{NMR}\left(50 \mathrm{MHz}, \mathrm{CDCl}_{3}\right.$ ): $141.0,127.5,127.4,126.6,97.0,89.5,52.1,50.4,48.7$, 29.5, 24.7, 23.5, 21.4, 9.3; ${ }^{11} \mathrm{~B}$ NMR (64 $\left.\mathrm{MHz} \mathrm{CDCl}_{3}\right): \square 32.4$.

\subsubsection{Dual auxiliary allylboronates $13 a$ and $13 b$}

A slurry of $\mathrm{CuBr} \cdot \mathrm{SMe}_{2}(422 \mathrm{mg}, 2.05 \mathrm{mmol})$ in THF $(3 \mathrm{~mL})$ at $0{ }^{\circ} \mathrm{C}$ under Ar was treated with $\mathrm{MeLi}\left(1.4 \mathrm{M}\right.$ in $\left.\mathrm{Et}_{2} \mathrm{O}, 2.9 \mathrm{~mL}, 4.1 \mathrm{mmol}\right)$. Once the colourless solution formed $(\sim 10 \mathrm{~min})$ the flask was placed in $\mathrm{a}-78{ }^{\circ} \mathrm{C}$ bath and treated via canula with a pre-cooled solution of chiral alkynoate 10e $(604 \mathrm{mg}, 2.02 \mathrm{mmol})$ in THF $(1 \mathrm{~mL},+1 \mathrm{~mL}$ canula rinse) and left to stir for $1.5 \mathrm{~h}$. The resulting dark mixture was then treated with the diisopropyl iodomethaneboronate $\mathbf{1 6 b}(1.59 \mathrm{~g}, 5.90 \mathrm{mmol})$ and HMPA $(3.6 \mathrm{~mL})$, left to stir for $5 \mathrm{~min}$ 
and then stirred at $0{ }^{\circ} \mathrm{C}$ for $2 \mathrm{~h}$. The reaction was then quenched with $\mathrm{NH}_{4} \mathrm{Cl}_{(\mathrm{aq})}(20 \mathrm{~mL})$ and the resulting biphasic mixture was divided into two roughly equal portions. One portion was poured into a solution of diol (-)-14 (250 mg, $1.02 \mathrm{mmol})$ in $\mathrm{Et}_{2} \mathrm{O}(10 \mathrm{~mL})$ and stirred for 30 min at RT. The layers were then separated and the aqueous phase was extracted with $\mathrm{Et}_{2} \mathrm{O}(3 \times 10 \mathrm{~mL})$. The combined organic phases were washed with water $(6 \times 10 \mathrm{~mL})$, brine $(10 \mathrm{~mL})$, dried $\left(\mathrm{Na}_{2} \mathrm{SO}_{4}\right)$ and concentrated to give the crude product $(722 \mathrm{mg})$. Flash chromatography $\left(10 \% \mathrm{Et}_{2} \mathrm{O} /\right.$ Hexanes $)$ gave the purified allylboronate 13a as a white solid $(511 \mathrm{mg}, 0.877 \mathrm{mmol})$. The second portion of the initial biphasic mixture was treated similarly with a solution of the enantiomeric diol (+)-14 $(254 \mathrm{mg}, 1.03 \mathrm{mmol})$ to give the diastereomeric allylboronate $\mathbf{1 3 b}(353 \mathrm{mg}, 0.606 \mathrm{mmol})$ as a colourless syrup after chromatography. The combined yield for the two isomers was $864 \mathrm{mg}$ (1.48 mmol, 73\%).

( $1 R ， 2 R ， 3 R ， 4 S)$-3-Methyl-2-(1,10,10-trimethyl-2-phenyl-3,5-dioxa-4-boratricyclo[5.2.1.0 $\left.{ }^{0,0}\right]$ dec-4-ylmethyl)-but-2-enoic acid, $(1 R, 2 S, 3 R)$-5-methyl-2-(1methyl-1-phenylethyl)cyclohexyl ester 13a: $\mathrm{mp}=50-55{ }^{\circ} \mathrm{C} ;{ }^{1} \mathrm{H}$ NMR $(500 \mathrm{MHz}$, $\left.\mathrm{CDCl}_{3}\right)$ : $7.43(\mathrm{~m}, 2 \mathrm{H}), 7.33(\mathrm{~m}, 2 \mathrm{H}), 7.23(\mathrm{~m}, 3 \mathrm{H}), 7.13(\mathrm{~m}, 3 \mathrm{H}), 4.68$ (dt, $J=10.7 \mathrm{~Hz}$, $4.3 \mathrm{~Hz}, 1 \mathrm{H}), 4.63(\mathrm{~s}, 1 \mathrm{H}), 2.09$ (d, $J=5.2 \mathrm{~Hz}, 1 \mathrm{H}), 2.05$ (s, 3H), 1.9-1.3 (m, 6H), 1.70 (s, $3 \mathrm{H}), 1.3-1.0(\mathrm{~m}, 4 \mathrm{H}), 1.20$ (s, 3H), 1.19 (s, 3H), 1.12 (s, 3H), 1.0-0.8 (m, 2H), 0.93 (s, 3H), $0.90(\mathrm{~s}, 3 \mathrm{H}), 0.75(\mathrm{~d}, J=6.6 \mathrm{~Hz}, 3 \mathrm{H}), 0.46(\mathrm{~m}, 2 \mathrm{H}) ;{ }^{13} \mathrm{C} \mathrm{NMR}\left(125 \mathrm{MHz}, \mathrm{CDCl}_{3}\right)$ : $\square$ 167.4, 151.2 , 144.3, 141.9, 128.0, 127.7, 127.5, 127.2, 126.9, 125.6, 125.5, 125.0, 123.5, 95.7, 88.6, 73.9, 52.0, 48.9, 41.5, 39.9, 34.1, 31.2, 29.6, 28.3, 26.9, 25.2, 24.8, 23.7, 23.6, 23.1, 21.7, 20.8, 9.4; ${ }^{11} \mathrm{~B}$ NMR (64 $\left.\mathrm{MHz}, \mathrm{CDCl}_{3}\right)$ : $\mathrm{n} 33.2$; IR $\left(\mathrm{CH}_{2} \mathrm{Cl}_{2}\right.$ cast, $\left.\mathrm{cm}^{-1}\right)$ : 3056, 2953, 1704, 1628, 1600, 1350, 1122, 760, 701; HRMS (EI, $m / z$ ) Calcd for $\mathrm{C}_{38} \mathrm{H}_{51}{ }^{11} \mathrm{BO}_{4}$, 582.38806, found 582.38910.

( $1 S, 2 S, 3 S, 4 R)$-3-Methyl-2-(1,10,10-trimethyl-2-phenyl-3,5-dioxa-4-boratricyclo[5.2.1.0 $\left.{ }^{0,0}\right]$ dec-4-ylmethyl)-but-2-enoic acid, $(1 R, 2 S, 3 R)$-5-methyl-2-(1methyl-1-phenylethyl)cyclohexyl ester 13b: ${ }^{1} \mathrm{H}$ NMR (500 MHz, $\left.\mathrm{CDCl}_{3}\right): \square .41$ (m, 2H), $7.30(\mathrm{~m}, 2 \mathrm{H}), 7.3-7.1(\mathrm{~m}, 6 \mathrm{H}), 4.75(\mathrm{dt}, J=10.8 \mathrm{~Hz}, 4.3 \mathrm{~Hz}, 1 \mathrm{H}), 4.70(\mathrm{~s}, 1 \mathrm{H}), 2.10$ (d, $J=5.2 \mathrm{~Hz}, 1 \mathrm{H}), 1.94(\mathrm{~s}, 3 \mathrm{H}), 1.8-0.8(\mathrm{~m}, 12 \mathrm{H}), 1.69(\mathrm{br} \mathrm{s}, 2 \mathrm{H}), 1.66(\mathrm{~s}, 3 \mathrm{H}), 1.21(\mathrm{~s}, 3 \mathrm{H})$, $1.16(\mathrm{~s}, 6 \mathrm{H}), 0.92(\mathrm{~s}, 3 \mathrm{H}), 0.91(\mathrm{~s}, 3 \mathrm{H}), 0.77(\mathrm{~d}, J=6.6 \mathrm{~Hz}, 3 \mathrm{H}) ;{ }^{13} \mathrm{C}$ NMR $(125 \mathrm{MHz}$, $\mathrm{CDCl}_{3}$ ): $\square 167.9,150.8,141.6,141.1,128.8,128.0,127.5,127.2,127.0,126.5,125.4,124.8$, 123.7, 95.6, 89.3, 88.5, 73.9, 51.9, 50.3, 50.0, 48.8, 41.6, 40.0, 34.4, 31.2, 29.6, 28.5, 27.0, 24.8, 23.6, 22.8, 21.8, 20.9, 9.4; ${ }^{11} \mathrm{~B}$ NMR (64 MHz, $\left.\mathrm{CDCl}_{3}\right)$ : $\square$ 35.4; IR $\left(\mathrm{CH}_{2} \mathrm{Cl}_{2}\right.$ cast, $\left.\mathrm{cm}^{-1}\right):$ 3056, 2955, 1704, 1600, 1346, 1121, 759, 701; HRMS (EI, $m / z$ ) Calcd for $\mathrm{C}_{38} \mathrm{H}_{51}{ }^{11} \mathrm{BO}_{4}, 582.38806$, found 582.38846. 


\subsection{3 (1R, 2R, 3R, 4S)-3-Methyl-2-(1,10,10-trimethyl-2-phenyl-3,5-dioxa-4-bora-}

\section{tricyclo[5.2.1.00,0]dec-4-ylmethyl)-but-2-enoic acid, ethyl ester 18}

A slurry of $\mathrm{CuBr} \cdot \mathrm{SMe}_{2}(738 \mathrm{mg}, 3.59 \mathrm{mmol})$ in THF $(10.5 \mathrm{~mL})$ at $0{ }^{\circ} \mathrm{C}$ under $\mathrm{Ar}$ was treated with $\mathrm{MeLi}\left(1.45 \mathrm{M}\right.$ in $\left.\mathrm{Et}_{2} \mathrm{O}, 4.9 \mathrm{~mL}, 7.1 \mathrm{mmol}\right)$. Once the colourless solution formed $(\sim 10 \mathrm{~min})$ the flask was placed in a $-78{ }^{\circ} \mathrm{C}$ bath and treated via canula with a pre-cooled solution of ethyl 2-butynoate $(400 \mathrm{mg}, 3.56 \mathrm{mmol})$ in THF $(1.8 \mathrm{~mL},+1.8 \mathrm{~mL}$ canula rinse) and left to stir for $1 \mathrm{~h}$. The resulting dark mixture was then treated with the diisopropyl iodomethaneboronate $\mathbf{1 6 b}(2.07 \mathrm{~g}, 7.67 \mathrm{mmol})$ and HMPA (5.6 mL, $32 \mathrm{mmol})$, left to stir for $5 \mathrm{~min}$ and then stirred at room temperature for $2 \mathrm{~h}$. The reaction was then quenched with $\mathrm{NH}_{4} \mathrm{Cl}_{\text {(aq) }}(30 \mathrm{~mL})$ and treated directly with a solution of diol (-)-14 (873 $\mathrm{mg}$, $3.54 \mathrm{mmol})$ in $\mathrm{Et}_{2} \mathrm{O}(30 \mathrm{~mL})$. The biphasic mixture was left to stir under Ar at room temperature for $2 \mathrm{~h}$, the layers were separated, and the aqueous phase was extracted with $\mathrm{Et}_{2} \mathrm{O}(3 \times 30 \mathrm{~mL})$. The combined ether layers were washed with water $(6 \times 30 \mathrm{~mL})$ and brine $(30 \mathrm{~mL})$, dried $\left(\mathrm{Na}_{2} \mathrm{SO}_{4}\right)$ and concentrated to give the crude product $(1.61 \mathrm{~g})$. Flash chromatography (10\% $\mathrm{Et}_{2} \mathrm{O} /$ Hexanes, $106 \mathrm{~g}$ silica) gave the pure compound (646 mg, $1.63 \mathrm{mmol}, 46 \%)$.

TLC (25\% Et 2 O/Hexanes, UV/ $\left.\mathrm{KMnO}_{4}\right)$ : 0.56; ${ }^{1} \mathrm{H}$ NMR (500 MHz, $\left.\mathrm{CDCl}_{3}\right): \square 7.38(\mathrm{~m}$, $2 \mathrm{H}), 7.30(\mathrm{~m}, 2 \mathrm{H}), 7.26(\mathrm{~m}, 1 \mathrm{H}), 4.69(\mathrm{~s}, 1 \mathrm{H}), 3.86(\mathrm{~m}, 1 \mathrm{H}), 3.73(\mathrm{~m}, 1 \mathrm{H}), 2.11(\mathrm{~d}$, $J=5.2 \mathrm{~Hz}, 1 \mathrm{H}), 2.02(\mathrm{~s}, 3 \mathrm{H}), 1.86(\mathrm{br} \mathrm{s}, 2 \mathrm{H}), 1.80(\mathrm{~m}, 1 \mathrm{H}), 1.72(\mathrm{~s}, 3 \mathrm{H}), 1.20(\mathrm{~s}, 3 \mathrm{H}), 1.15$ $(\mathrm{m}, 2 \mathrm{H}), 1.01(\mathrm{~m}, 1 \mathrm{H}), 0.93(\mathrm{t}, J=7.1 \mathrm{~Hz}, 3 \mathrm{H}), 0.92(\mathrm{~s}, 3 \mathrm{H}), 0.90(\mathrm{~s}, 3 \mathrm{H}) ;{ }^{13} \mathrm{C}$ NMR (125 MHz, $\mathrm{CDCl}_{3}$ ): $\square 168.8,142.8,141.8,127.3,127.1,126.7,123.3,95.6,88.8,59.7,51.9$, 50.1, 48.9, 29.6, 24.7, 23.6, 23.0, 22.9, 20.7, 13.9, 9.3; ${ }^{11} \mathrm{~B}$ NMR (64 MHz, $\mathrm{CDCl}_{3}$ ): $\square 33.2$; IR $\left(\mathrm{CH}_{2} \mathrm{Cl}_{2}\right.$ cast, $\left.\mathrm{cm}^{-1}\right): 2956,1713,1633,1345,1121,759,702$; HRMS (EI, $\left.\mathrm{m} / z\right)$ Calcd for $\mathrm{C}_{24} \mathrm{H}_{33}{ }^{11} \mathrm{BO}_{4}, 396.24719$, found 396.24821 .

\subsubsection{Preparation of enantioenriched lactones $2 \mathrm{~b}, 2 \mathrm{~d}$ and 20 from dual auxiliary allylboronates 13}

\subsubsection{Enantioenriched 4,4-dimethyl-3-methylene-5-nonyl-dihydro-furan-2-one $2 \mathrm{~d}$ from dual auxiliary allylboronate $13 a-$ Matched Case}

Allylboronate 13a (213 mg, $0.366 \mathrm{mmol})$ and decanal $(50 \mathrm{mg}, 0.32 \mathrm{mmol})$ were stirred together in toluene $(0.6 \mathrm{~mL})$ at room temperature for $18 \mathrm{~d}$. A spatula tip of pTSA was then added and the mixture was left to stir for $3 \mathrm{~h}$. The reaction was then quenched with $\mathrm{NaHCO}_{3(\text { aq })}(5 \mathrm{~mL})$ and extracted with $\mathrm{Et}_{2} \mathrm{O}(3 \times 5 \mathrm{~mL})$. The combined organic layers 
were washed with brine $(5 \mathrm{~mL})$, dried $\left(\mathrm{Na}_{2} \mathrm{SO}_{4}\right)$ and concentrated to give the crude lactone 2d (240 mg), which was then purified by flash chromatography (1\% EtOAc/toluene, $28 \mathrm{~g}$ silica) to give the pure compound $(73 \mathrm{mg}, 0.29 \mathrm{mmol}, 90 \%)$. Enantiomeric excess (CHIRALPAK AD-RH, $50 \%$ isopropanol/water, $0.3 \mathrm{~mL} / \mathrm{min}$ ): $98 \%$ for the $(S)$-enantiomer.
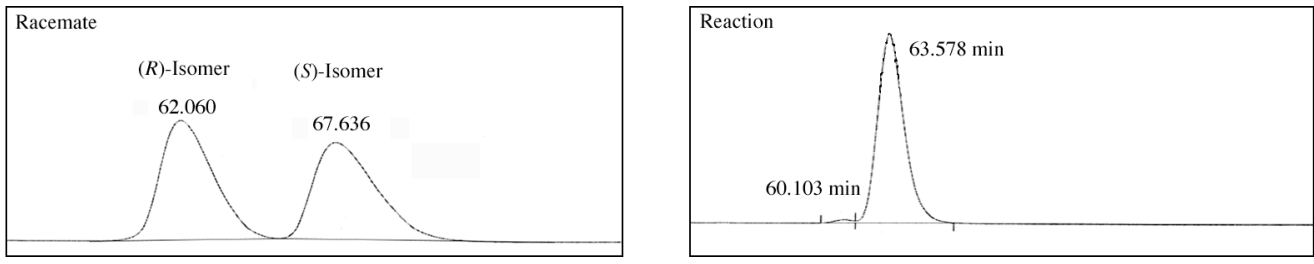

\subsubsection{Enantioenriched 4,4-dimethyl-3-methylene-5-nonyl-dihydro-furan-2-one $2 \mathrm{~d}$ from dual auxiliary allylboronate $13 b$ - Mis-matched Case}

This reaction was carried out as described in Section 5.3.4.1 with allylboronate $\mathbf{1 3 b}$ (306 $\mathrm{mg}, 0.524 \mathrm{mmol}$ ) and decanal $(82 \mathrm{mg}, 0.52 \mathrm{mmol})$ to give $20 \mathrm{mg}$ of lactone $\mathbf{2 d}$ (0.079 mmol, 15\%). Chiral HPLC (CHIRALPAK AD-RH, 50\% isopropanol/water, $0.3 \mathrm{~mL} / \mathrm{min}$ ) showed that the product was essentially racemic.
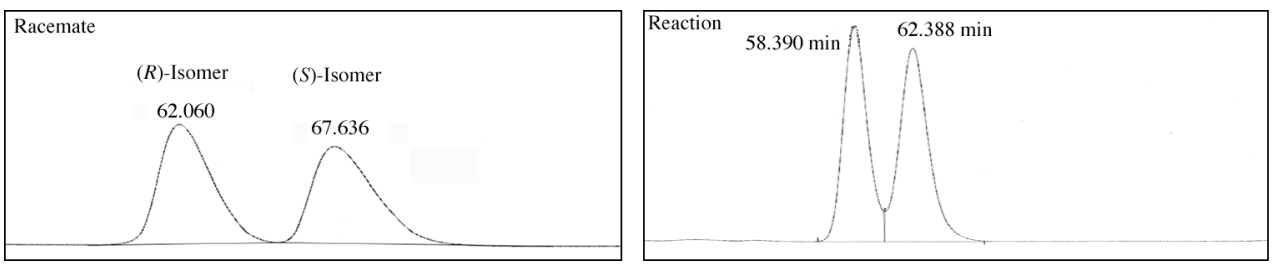

\subsubsection{Enantioenriched 4,4-dimethyl-3-methylene-5-nonyl-dihydro-furan-2-one $2 \mathrm{~d}$ from ¿-naphthylmenthol allylboronate $11 f$}

This reaction was carried out as described in Section 5.3.4.1 with allylboronate $11 \mathbf{f}$ (402 $\mathrm{mg}, 0.797 \mathrm{mmol}$ ) and decanal $(85 \mathrm{mg}, 0.54 \mathrm{mmol}$ ) to give $28 \mathrm{mg}$ of lactone $2 \mathbf{d}$ (0.11 mmol, 20\%). Enantiomeric excess (CHIRALPAK AD-RH, 50\% isopropanol/water, $0.3 \mathrm{~mL} / \mathrm{min}): 82 \%$ for the $(S)$-enantiomer.
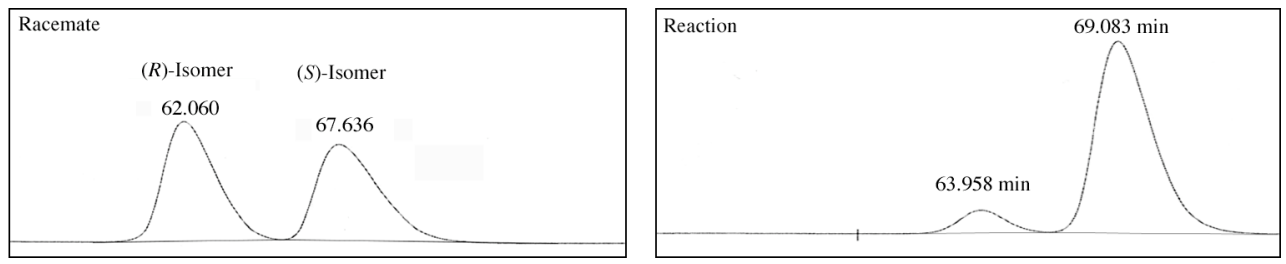


\subsubsection{Enantioenriched 4,4-dimethyl-3-methylene-5-nonyl-dihydro-furan-2-one $2 \mathrm{~d}$ from chiral allylboronate 18}

This reaction was carried out as described in Section 5.3.4.1 with allylboronate $\mathbf{1 8}$ (136 mg, $0.354 \mathrm{mmol})$ and decanal $(42 \mathrm{mg}, 0.27 \mathrm{mmol})$. The enantiomeric excess was determined from the crude product 36a, resulting in a chromatogram with three peaks in the region of interest. The peaks due the two enantiomers of the product were identified by co-injecting the product from the reaction with a sample of the racemate. Enantiomeric excess (CHIRALPAK AD-RH, 50\% isopropanol/water, $0.3 \mathrm{~mL} / \mathrm{min}$ ): $51 \%$ for the (S)-enantiomer.
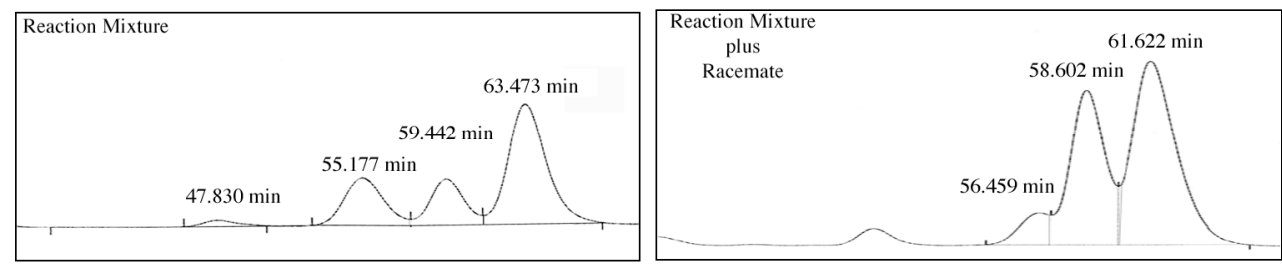

\subsubsection{Enantioenriched 4,4-dimethyl-3-methylene-5-phenyl-dihydro-furan-2-one $2 \mathrm{~b}$ from dual auxiliary allylboronate $13 a$}

This reaction was carried out as described in Section 5.3.4.1 with allylboronate 13a (172 $\mathrm{mg}, 0.295 \mathrm{mmol}$ ) and benzaldehyde $(32 \mathrm{mg}, 0.30 \mathrm{mmol})$. Flash chromatography (2.5\% EtOAc/Toluene, $30 \mathrm{~g}$ silica) gave the product $\mathbf{2 b}$ that was contaminated with both auxiliaries (33 mg, 55\%). Enantiomeric excess (CHIRALCEL OD, $0.5 \%$ isopropanol/pentane, $1 \mathrm{~mL} / \mathrm{min})$ : $>95 \%$ for the $(S)$-enantiomer. The preparation of the racemate is described in Section 5.1.2 (page 18).
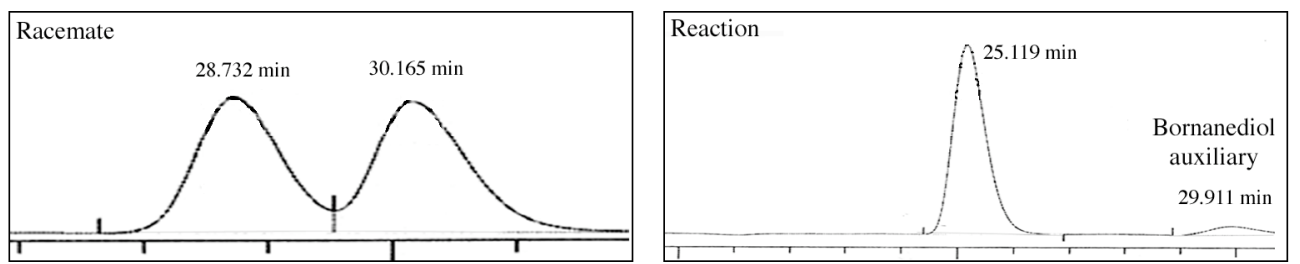


\subsubsection{Enantioenriched 5-(4-bromophenyl)-4,4-dimethyl-3-methylene-dihydro-furan-2-one 20 from dual auxiliary allylboronate $13 a$}

This reaction was carried out as described in Section 5.3.4.1 with allylboronate 13a (98 mg, $0.168 \mathrm{mmol}$ ) and $p$-bromobenzaldehyde (48 $\mathrm{mg}, 0.26 \mathrm{mmol}$ ). Work-up gave the crude product as an oil (100 mg). Flash chromatography (1\% EtOAc/Toluene, $13 \mathrm{~g}$ silica) gave a small fraction of the pure product $20(5 \mathrm{mg})$ and another fraction of product that was contaminated with 8-phenylmenthol $(15 \mathrm{mg})$. Total yield: $20 \mathrm{mg}, 0.068 \mathrm{mmol}, 40 \%$. The contaminated product was dissolved in pentane and chilled in the freezer to produce X-ray quality crystals. Enantiomeric excess (CHIRALCEL OD, 2\% isopropanol/hexane, $1 \mathrm{~mL} / \mathrm{min})$ : $>95 \%$ for the $(S)$-enantiomer. The preparation of the racemate is described in Section 6.4.2 (page 54).
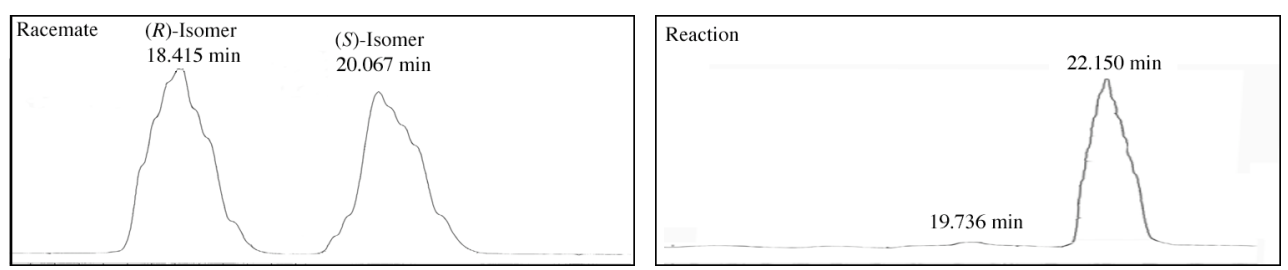


\section{Lewis acid catalysed allylborations with 2-carboxyester}

\section{3,3-disubstituted allylboronates 1}

\subsection{General procedure for NMR catalyst search}

The reaction with scandium triflate is representative. An NMR tube was charged with $\mathrm{Sc}(\mathrm{OTf})_{3}(9 \mathrm{mg}, 0.02 \mathrm{mmol})$ and dried overnight under vacuum at $130{ }^{\circ} \mathrm{C}$. Benzaldehyde ( $5 \mathrm{~L}, 0.05 \mathrm{mmol}$ ) was then added, followed by a $0.07 \mathrm{M}$ solution of allylboronate $1 \mathrm{c}$ in $\mathrm{CD}_{2} \mathrm{Cl}_{2}(0.75 \mathrm{~mL}, 0.053 \mathrm{mmol}) .{ }^{1} \mathrm{H}$ and ${ }^{11} \mathrm{~B} \mathrm{NMR}$ spectra were recorded daily for 7 days. The quartet at $4.14 \mathrm{ppm}\left(\mathrm{O}-\mathrm{CH}_{2}-\mathrm{Me}\right.$ for allylboronate 1c) and the singlet at $6.32 \mathrm{ppm}$ (olefin proton for lactone $\mathbf{2 f}$ ) were used as reference peaks in the proton spectra. Some ${ }^{1} \mathrm{H}$ spectra were complicated by the paramagnetic nature of some of the catalysts. To remove any adventitious triflic acid, one NMR reaction was run using $\mathrm{Sc}(\mathrm{OTf})_{3}$, allylboronate $1 \mathrm{c}\left(12.8 \mathrm{mg}\right.$ in $0.75 \mathrm{~mL}$ of $\left.\mathrm{CD}_{2} \mathrm{Cl}_{2}\right)$, benzaldehyde $(4.5 \square \mathrm{L}$, $0.044 \mathrm{mmol})$, and diisopropylamine (4.2 $\square \mathrm{L}, 0.024 \mathrm{mmol})$.

Studies with allylboronate $\mathbf{2 4}$ were performed in an analogous manner, except that the spectra were recorded every hour for 7 hours, reflecting the increased reactivity of these allylboronates. $^{1 \mathrm{~b}}$

The half-life reaction time for the $\mathrm{Sc}(\mathrm{OTf})_{3}$-catalyzed reaction was taken directly from Figure 7a. The data for the background uncatalysed run were extrapolated from an expanded version of this graph and assumed plot linearity. This analysis constitutes an underestimation given the expected exponential shape of the plot. These data were collected under the NMR screening conditions described previously of $50 \mathrm{~mol} \% \mathrm{Sc}(\mathrm{OTf})_{3}$. 


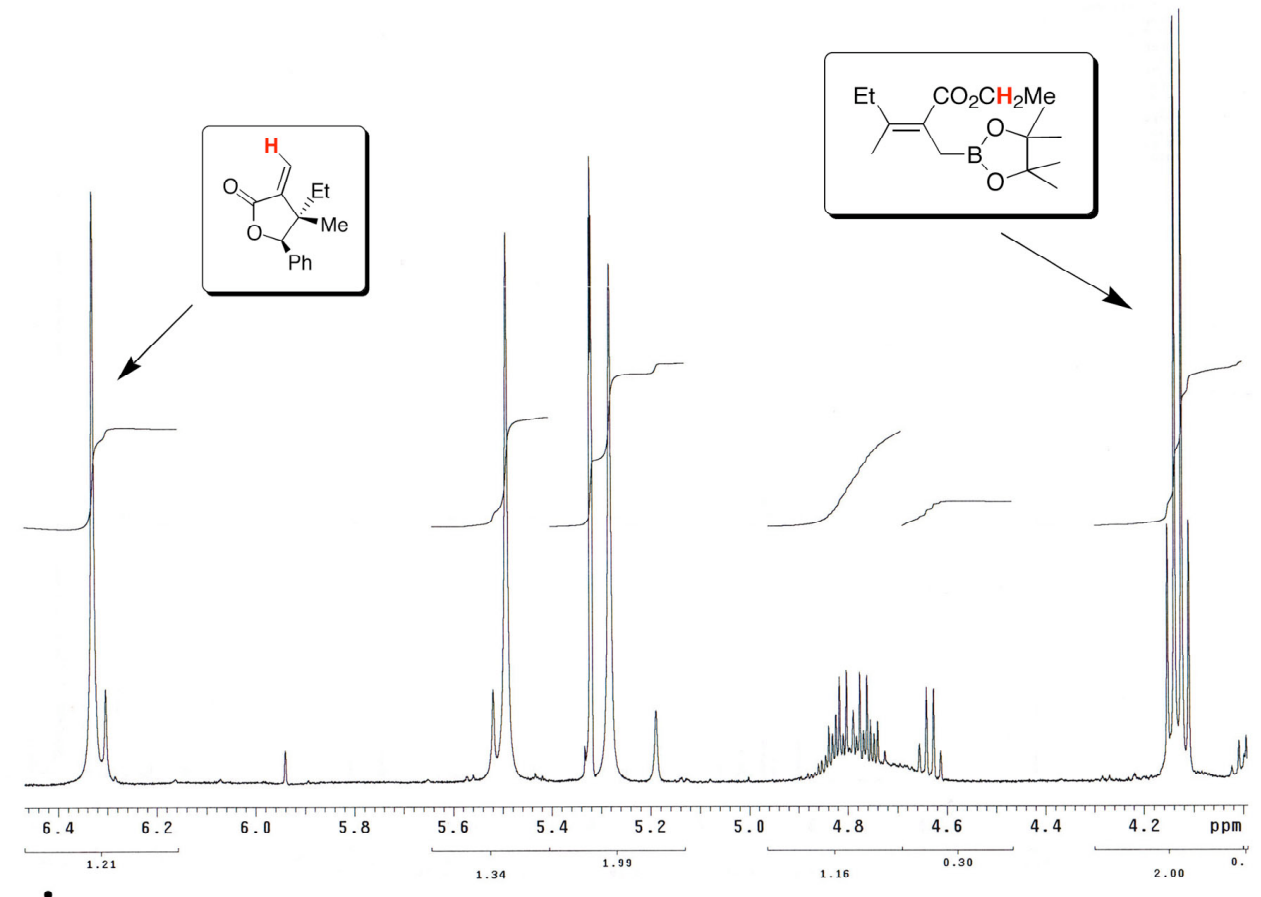

Figure S-1. Representative ${ }^{1} \mathrm{H}$ NMR Spectrum for $\mathbf{1 c / 2 f}\left(\mathrm{CD}_{2} \mathrm{Cl}_{2}, 500 \mathrm{MHz}\right)$.

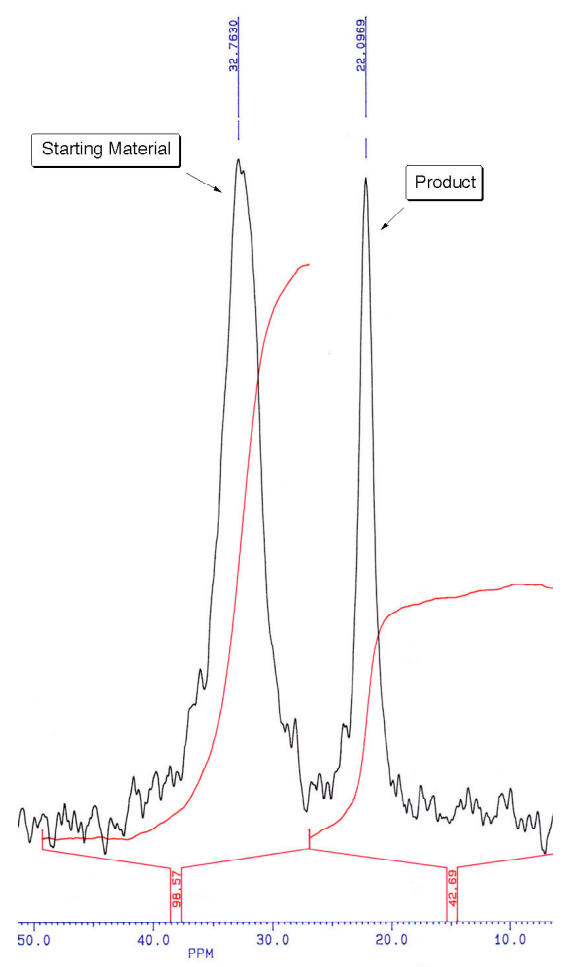

Figure S-2. Representative ${ }^{11} \mathrm{~B}$ NMR Spectrum for $1 \mathrm{c} / 20\left(\mathrm{CD}_{2} \mathrm{Cl}_{2}, 64 \mathrm{MHz}\right)$. 


\subsection{Optimization and control experiments for Lewis acid catalysed allylboration}

\subsubsection{Optimization of the metal ion}

a)

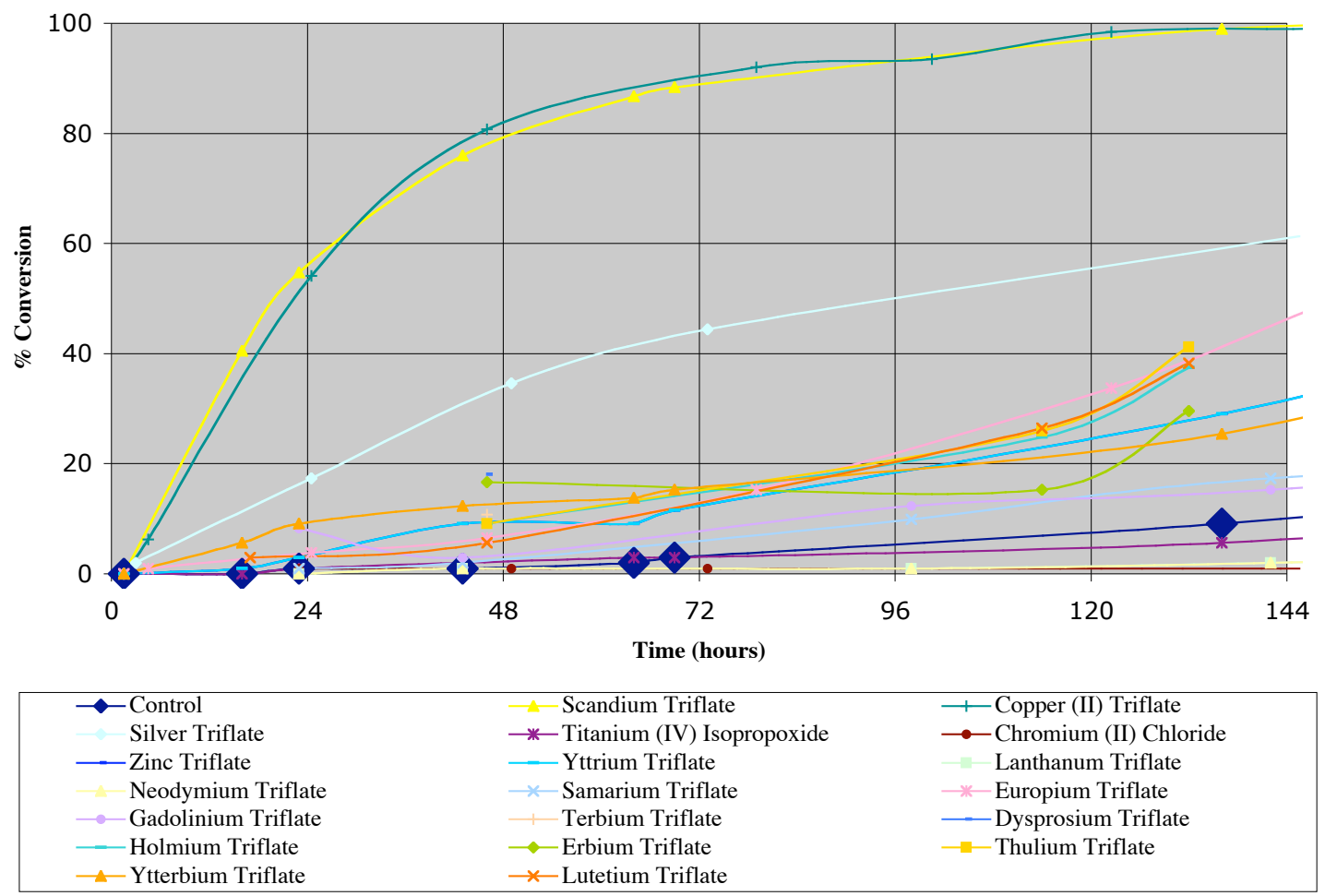


b)

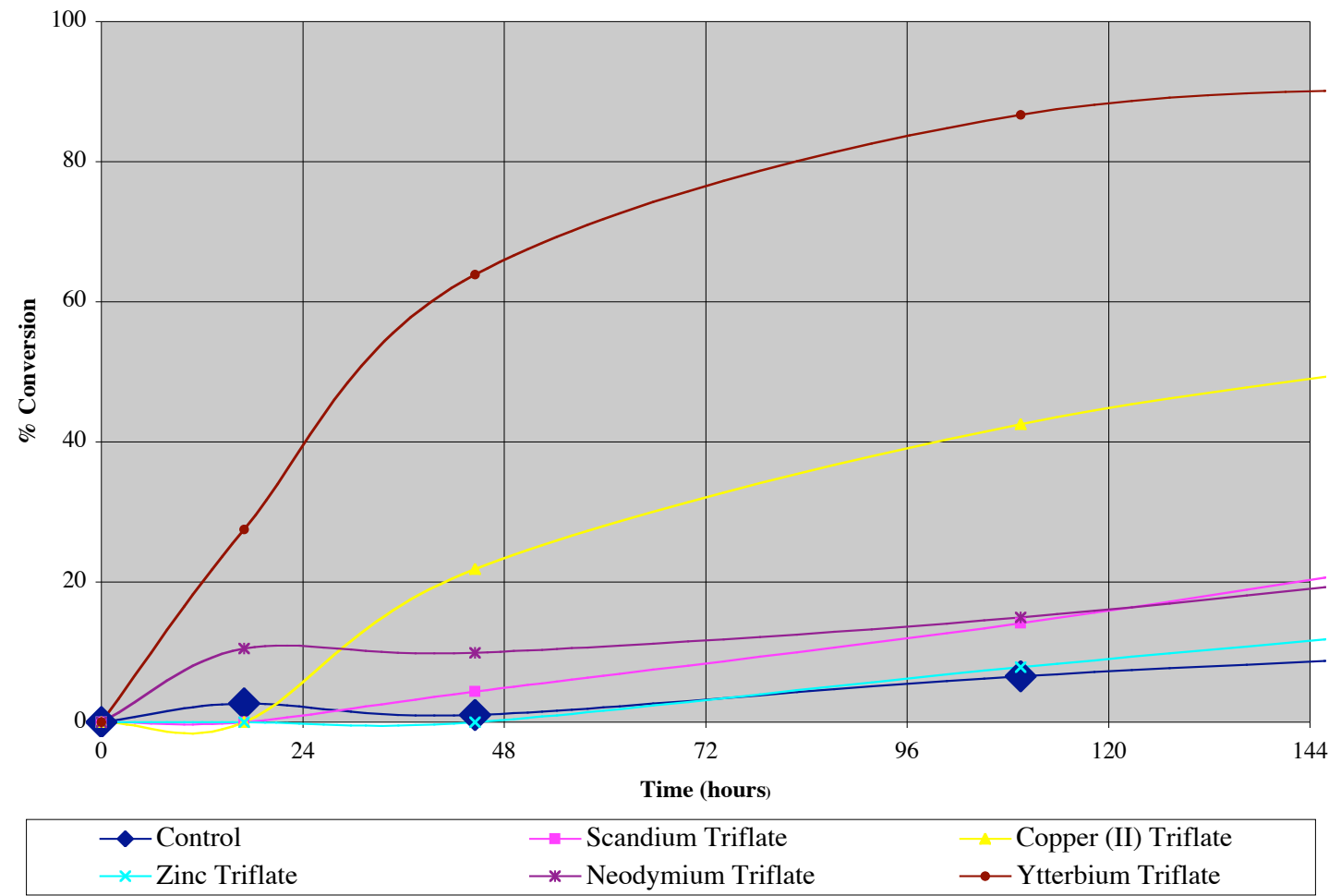

Initial metal screening for allylboration catalysts. Control reaction shown with extra large diamonds. Data collected from ${ }^{1} \mathrm{H}$ NMR data (400 MHz). a) Reactions in $\mathrm{CD}_{2} \mathrm{Cl}_{2}$. b) Reactions in 1:1 THF- $d_{8}: \mathrm{CD}_{2} \mathrm{Cl}_{2}$. 


\subsubsection{Optimization of the counter ion}
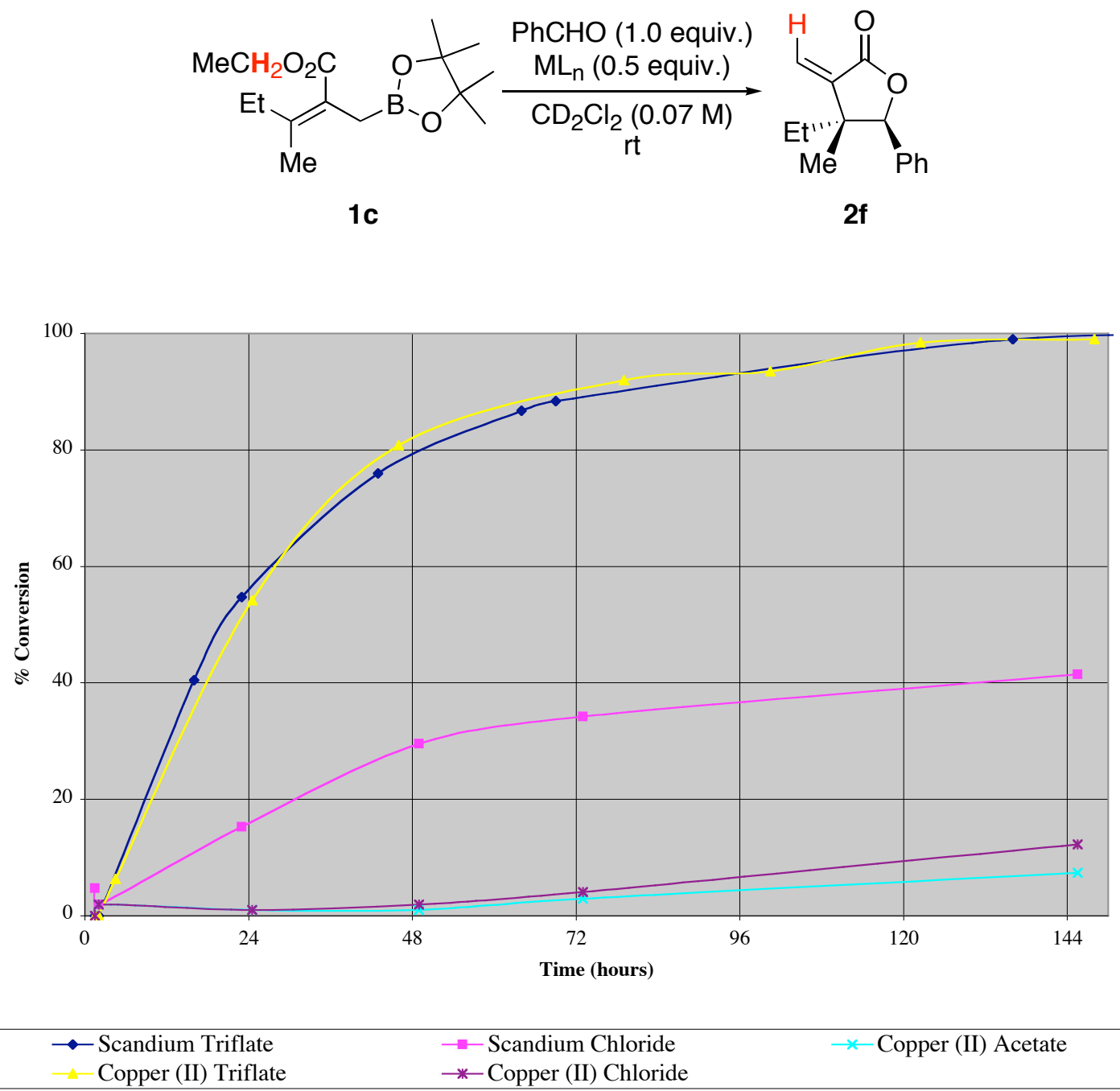


\subsubsection{Optimization of the solvent}
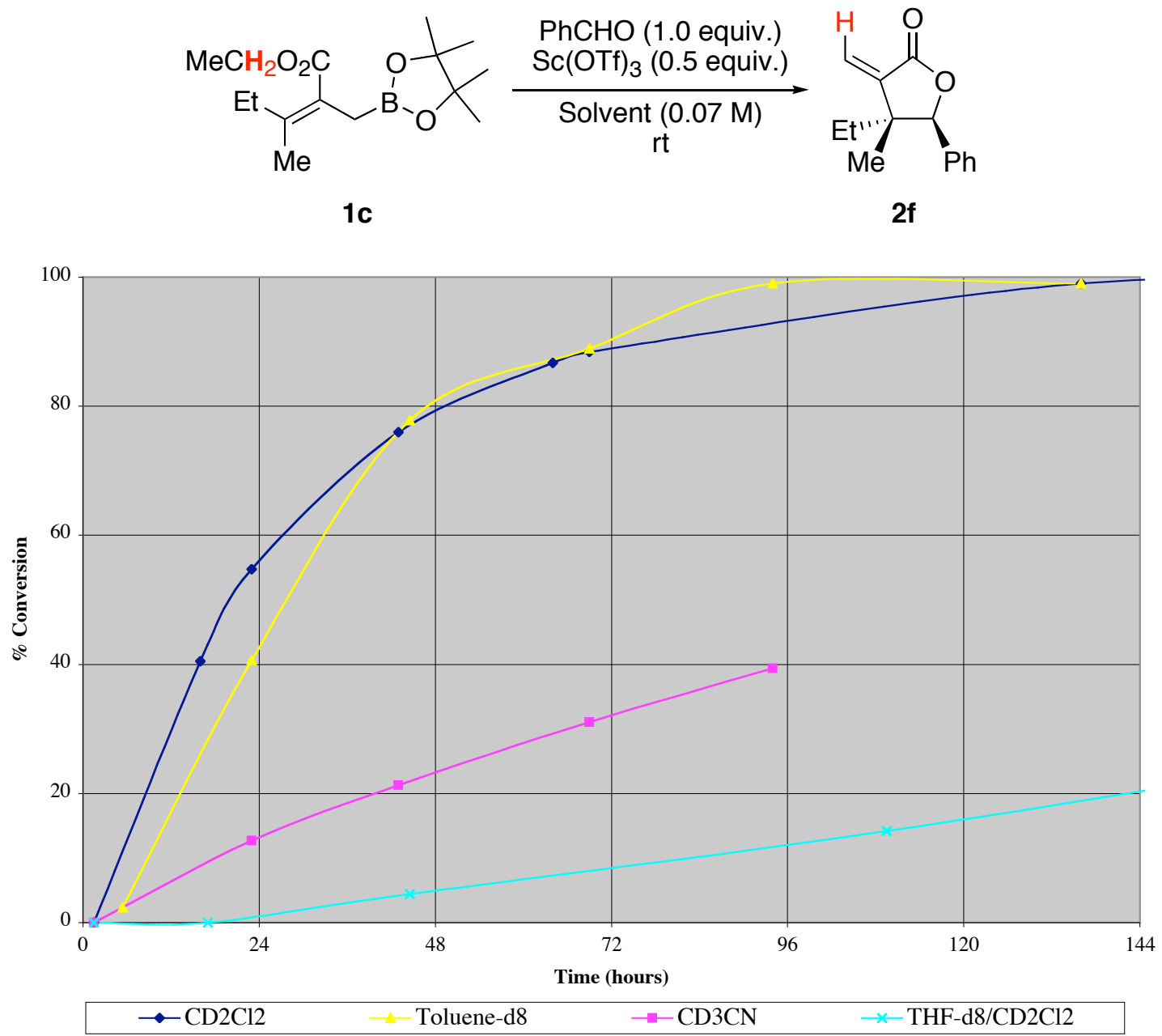


\subsubsection{Catalyst-control reactions}

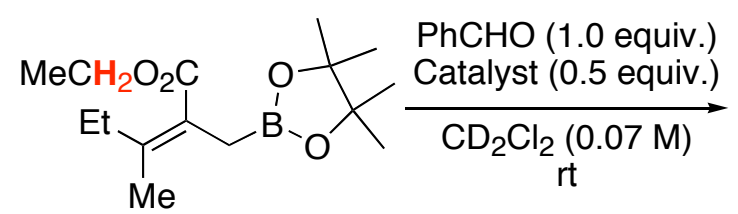

$1 c$

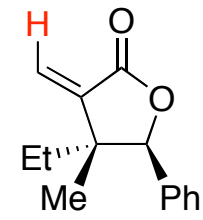

$2 f$

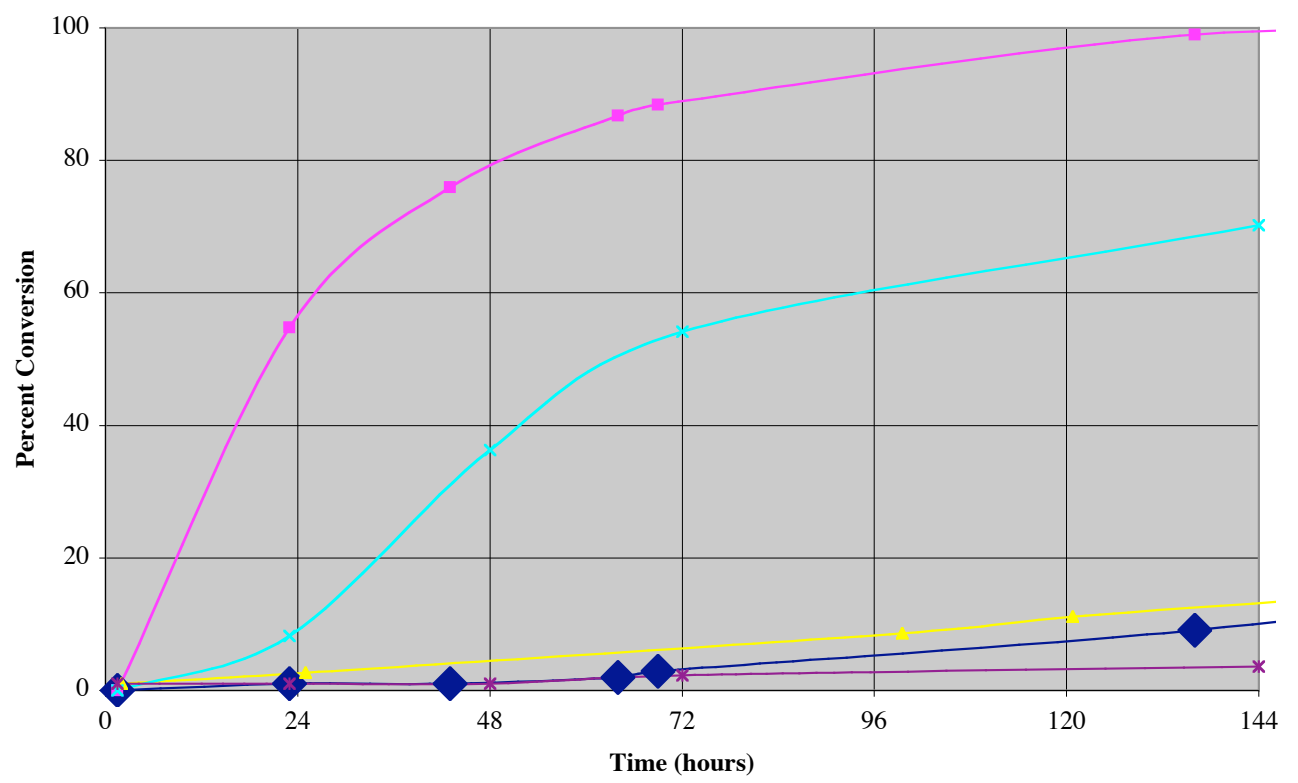

$\multimap$ Control $\rightarrow-$ Scandium Triflate $\longrightarrow$ Tetrabutylammonium triflate $\multimap$ Sc(OTf)3 : (i)-Pr2NH $\rightarrow-(\mathrm{i})-\operatorname{Pr} 2 \mathrm{NH}$ 
$6.3{ }^{1} \mathrm{H}$ and ${ }^{11} \mathrm{~B}$ NMR spectra of mixtures of allylboronate $1 \mathrm{c}$ and $\mathrm{Sc}(\mathrm{OTf})_{3}$

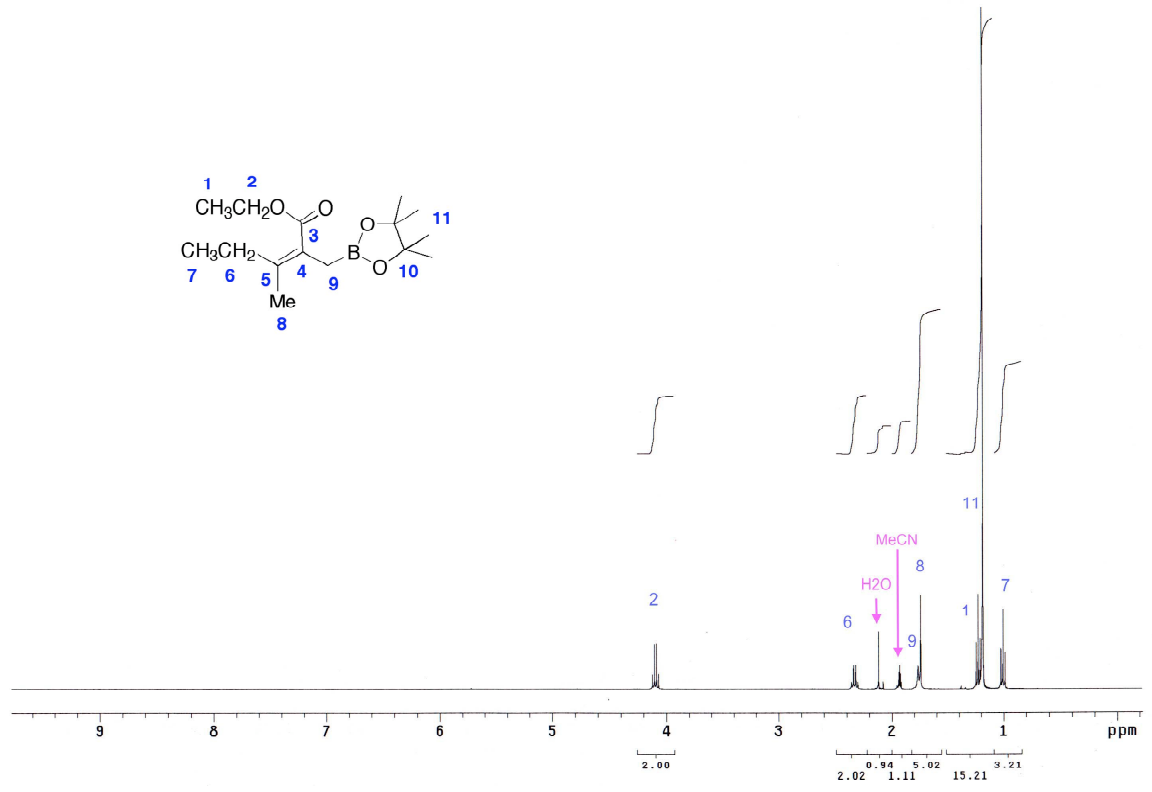

Figure S-3. ${ }^{1} \mathrm{H}$ NMR spectrum of allylboronate $1 \mathrm{c}$ in $\mathrm{CD}_{3} \mathrm{CN}(400 \mathrm{MHz})$. 

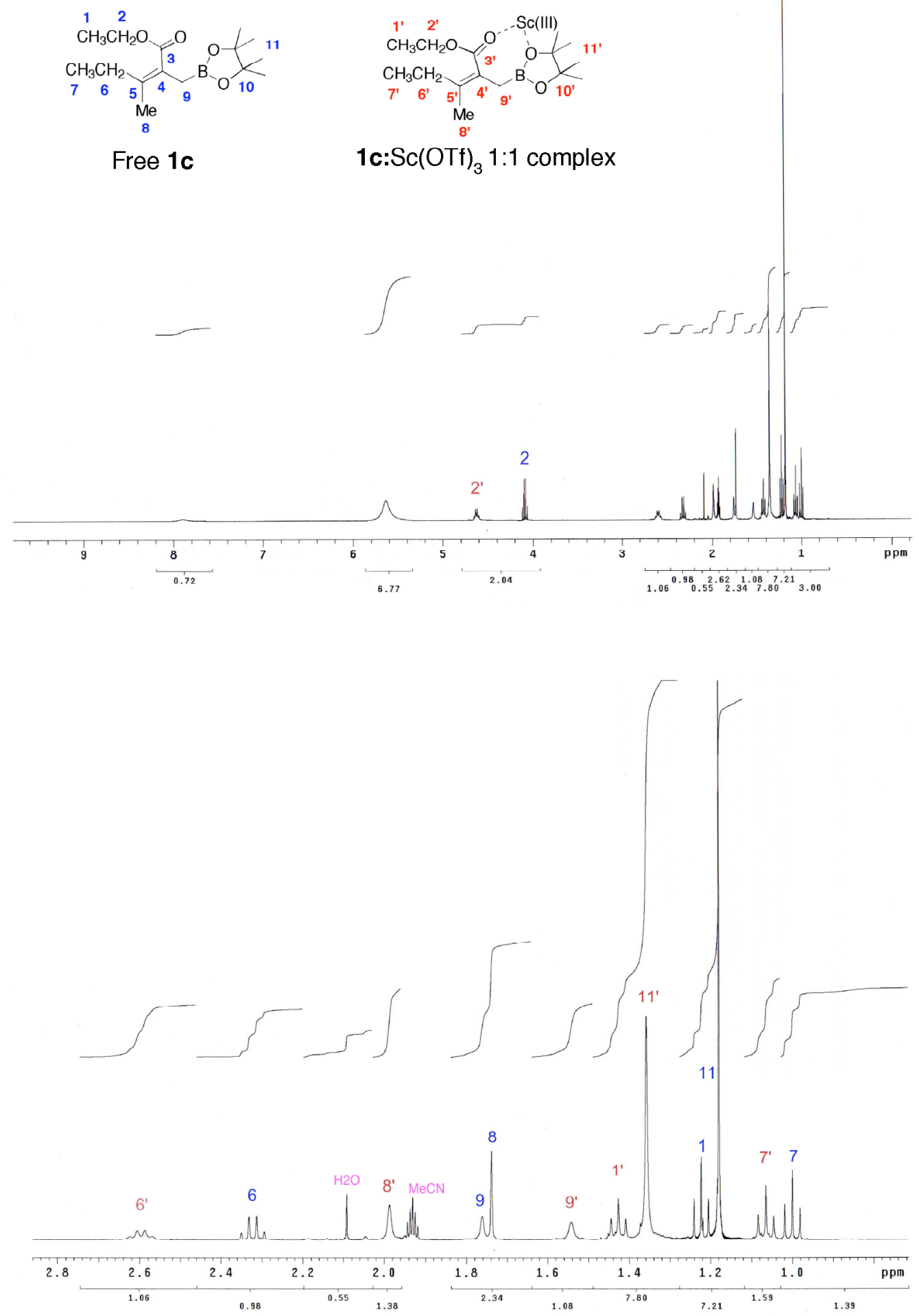

Figure S-4. ${ }^{1} \mathrm{H}$ NMR spectrum of allylboronate 1c with 1 equivalent of $\mathrm{Sc}(\mathrm{OTf})_{3}$ in $\mathrm{CD}_{3} \mathrm{CN}(400 \mathrm{MHz})$ with expansion. The structure of the allylboronate:scandium complex shown is hypothetical. 


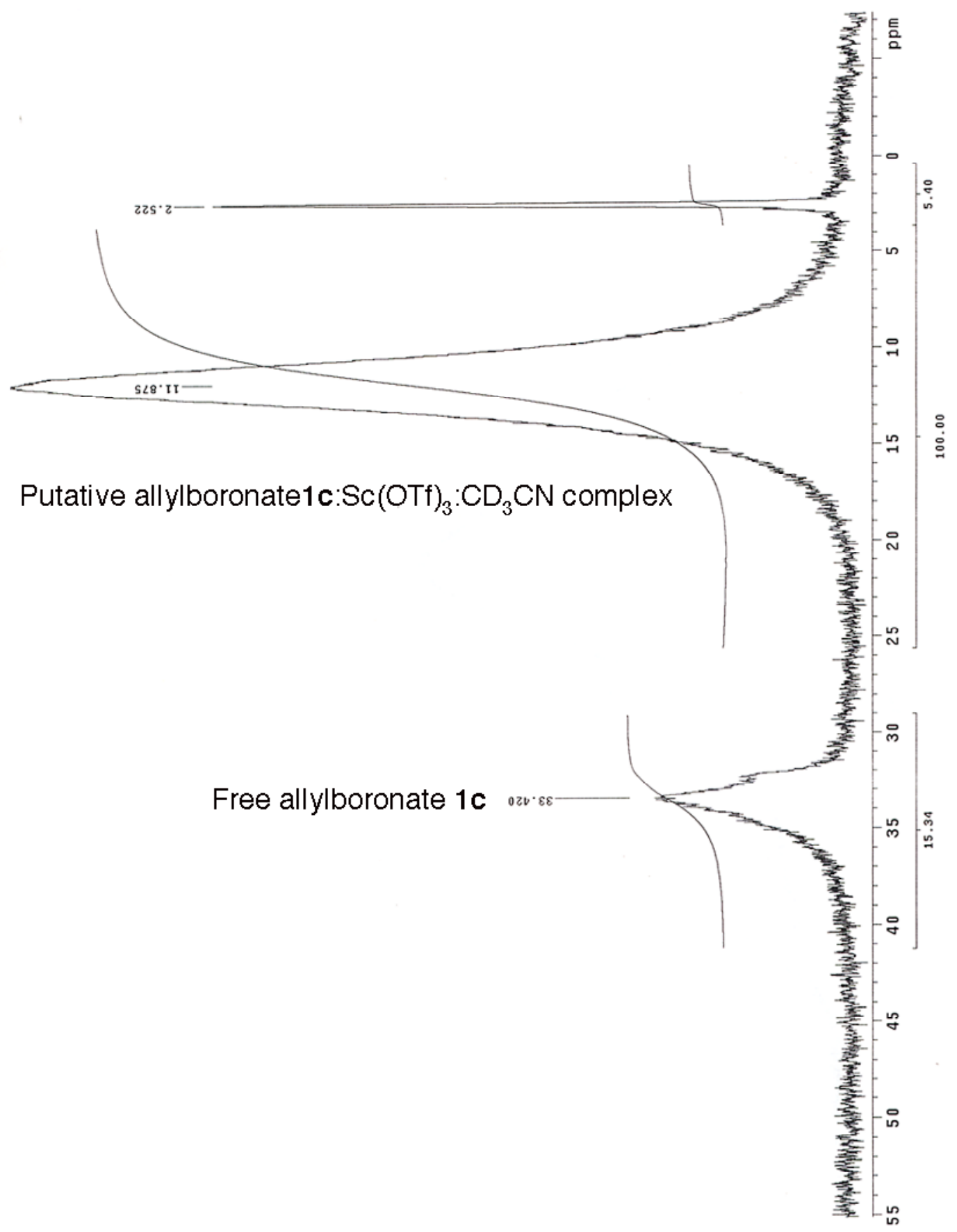

Figure S-5. ${ }^{11} \mathrm{~B}$ NMR spectrum of allylboronate 1c with 2 equivalents of $\mathrm{Sc}(\mathrm{OTf})_{3}$ in $\mathrm{CD}_{3} \mathrm{CN}$ (128 MHz). 


\subsection{Catalysed allylborations with aromatic aldehydes}

\subsection{1 $\left(4 R^{*}, 5 S^{\star}\right)-4$-Ethyl-4-methyl-3-methylene-5-phenyl-dihydro-furan-2-one $2 f$}

A slurry of allylboronate 1c $(140 \mathrm{mg}, 0.495 \mathrm{mmol})$, benzaldehyde $(55 \mathrm{mg}$, $0.52 \mathrm{mmol})$ and $\mathrm{Sc}(\mathrm{OTf})_{3}(25 \mathrm{mg}, 0.052 \mathrm{mmol})$ was stirred in toluene $(0.5 \mathrm{~mL})$ at room temperature under Argon for $24 \mathrm{~h}$. The mixture was then diluted with $\mathrm{NH}_{4} \mathrm{Cl} / \mathrm{NH}_{4} \mathrm{OH}(9: 1$, $\mathrm{v} / \mathrm{v}, 5 \mathrm{~mL})$ and extracted with $\mathrm{Et}_{2} \mathrm{O}(3 \times 5 \mathrm{~mL})$. The combined extractions were washed with brine $(5 \mathrm{~mL})$, dried $\left(\mathrm{Na}_{2} \mathrm{SO}_{4}\right)$ and concentrated to give the product $(99 \mathrm{mg}, 0.46 \mathrm{mmol}$, 93\%). Similarly, reactions using $\mathrm{Cu}(\mathrm{OTf})_{2}$ and $\mathrm{Yb}(\mathrm{OTf})_{3}$ gave the product in yields of $67 \%$ and $91 \%$ respectively after flash chromatography. The spectroscopic data for this compound were identical to that described in Section 5.1.6 (page 20).

\subsection{2 $\left(4 R^{*}, 5 S^{*}\right)$-5-(4-Bromophenyl)-4,4-dimethyl-3-methylene-dihydro-furan-2-one 20}

A solution of allylboronate 1c $(105 \mathrm{mg}, 0.39 \mathrm{mmol})$ and 4-bromobenzaldehyde $(63 \mathrm{mg}, 0.34 \mathrm{mmol})$ in toluene $(0.4 \mathrm{~mL})$ was treated with $\mathrm{Sc}(\mathrm{OTf})_{3}(18 \mathrm{mg}, 0.037 \mathrm{mmol})$ and stirred at room temperature under Ar for $19 \mathrm{~h}$. The reaction was then diluted with water $(5 \mathrm{~mL})$ and extracted with $\mathrm{Et}_{2} \mathrm{O}(3 \times 5 \mathrm{~mL})$. The combined extracts were washed with brine $(5 \mathrm{~mL})$, dried $\left(\mathrm{Na}_{2} \mathrm{SO}_{4}\right)$, and concentrated to give the crude product as an oil $(115 \mathrm{mg})$. Flash chromatography (5\% EtOAc/Toluene, $9 \mathrm{~g} \mathrm{SiO}_{2}$ ) yielded the pure product (60 $\mathrm{mg}$, $0.22 \mathrm{mmol}, 62 \%)$.

TLC (5\% EtOAc/Toluene, UV/ $\mathrm{KMnO}_{4}$ ): 0.40; ${ }^{1} \mathrm{H}$ NMR (500 MHz, $\left.\mathrm{CDCl}_{3}\right)$ : $7.52(\mathrm{AB}$, $J=8.5 \mathrm{~Hz}, 2 \mathrm{H}), 7.14(\mathrm{AB}, J=8.7 \mathrm{~Hz}, 2 \mathrm{H}), 6.28(\mathrm{~s}, 1 \mathrm{H}), 5.56(\mathrm{~s}, 1 \mathrm{H}), 5.10(\mathrm{~s}, 1 \mathrm{H}), 1.38$ (s, $3 \mathrm{H}), 0.74$ (s, 3H); ${ }^{13} \mathrm{C}$ NMR (125 MHz, $\mathrm{CDCl}_{3}$ ): $\square 169.9,145.1,134.8,131.6,127.5,122.4$, 120.5, 87.3, 43.5, 25.7, 24.3; IR $\left(\mathrm{CH}_{2} \mathrm{Cl}_{2}\right.$ cast, $\left.\mathrm{cm}^{-1}\right): 2966,1770,1660,1594,1118,814$; HRMS (EI, $m / z$ ) Calcd for $\mathrm{C}_{13} \mathrm{H}_{13} \mathrm{O}_{2}{ }^{81} \mathrm{Br}$ : 282.00784. Found: 282.00818; Calcd for $\mathrm{C}_{13} \mathrm{H}_{13} \mathrm{O}_{2}{ }^{79} \mathrm{Br}$ : 280.00989. Found: 280.00991 .

\subsection{3 $\left(4 R^{\star}, 5 S^{\star}\right)$-5-(4-Acetoxyphenyl )-4-ethyl-4-methyl-3-methylene-dihydro-furan-2-one $2 \mathrm{p}$}

A solution of allylboronate 1c $(62 \mathrm{mg}, 0.22 \mathrm{mmol})$ and $p$-acetoxybenzaldehyde $(33 \mathrm{mg}, 0.20 \mathrm{mmol})$ in toluene $(0.2 \mathrm{~mL})$ was treated with $\mathrm{Sc}(\mathrm{OTf})_{3}(12 \mathrm{mg}, 0.024 \mathrm{mmol})$ and stirred at room temperature under Ar for $18 \mathrm{~h}$. Work up as described in Section 6.4.2 gave the product as a crude oil $(71 \mathrm{mg})$. Flash chromatography (2.5\% Acetone/Toluene, 
$4 \mathrm{~g} \mathrm{SiO}_{2}$ ) gave the product ( $35 \mathrm{mg}, 0.13 \mathrm{mmol}, 64 \%$ ), which was further purified by Kugelrohr distillation $\left(250{ }^{\circ} \mathrm{C}, 0.1\right.$ torr) to give the pure lactone (32 mg, $0.12 \mathrm{mmol}, 57 \%$ ).

TLC (2.5\% Acetone/Toluene, UV/ $\mathrm{KMnO}_{4}$ ): $0.21 ;{ }^{1} \mathrm{H} \mathrm{NMR}\left(500 \mathrm{MHz}, \mathrm{CDCl}_{3}\right): \square 7.14$ $(\mathrm{AB}, J=8.3 \mathrm{~Hz}, 2 \mathrm{H}), 7.07(\mathrm{AB}, J=8.3 \mathrm{~Hz}, 2 \mathrm{H}), 6.34(\mathrm{~s}, 1 \mathrm{H}), 5.45(\mathrm{~s}, 1 \mathrm{H}), 5.25(\mathrm{~s}, 1 \mathrm{H})$, $2.27(\mathrm{~s}, 3 \mathrm{H}), 1.68(\mathrm{~m}, 2 \mathrm{H}), 0.96(\mathrm{t}, J=7.5 \mathrm{~Hz}, 3 \mathrm{H}), 0.73(\mathrm{~s}, 3 \mathrm{H}) ;{ }^{13} \mathrm{C} \mathrm{NMR}(125 \mathrm{MHz}$, $\left.\mathrm{CDCl}_{3}\right): \square 170.4,169.0,150.5,142.6,134.4,127.0,121.7,121.5,85.7,47.0,33.4,22.6$, 21.2, 8.6; Anal. Calcd for $\mathrm{C}_{16} \mathrm{H}_{18} \mathrm{O}_{4}$ : C, 70.04; H, 6.63. Found: C, 69.62; H, 6.40.

\subsection{4 (4R $\left(4 R^{\star}, 5 R^{\star}\right)-4-B u t y l-5$-(3-iodophenyl)-4-methyl-3-methylene-dihydro-furan-2-one $2 q$}

A solution of allylboronate 1e $(124 \mathrm{mg}, 0.401 \mathrm{mmol})$ and 3-iodobenzaldehyde $(142 \mathrm{mg}, 0.614 \mathrm{mmol})$ in toluene $(0.4 \mathrm{~mL})$ was treated with $\mathrm{Sc}(\mathrm{OTf})_{3}(20 \mathrm{mg}, 0.041 \mathrm{mmol})$. The resulting slurry was stirred at room temperature under Ar for $24 \mathrm{~h}$. Work up as described in Section 6.4.2 gave the product as a crude oil $(280 \mathrm{mg})$. Flash chromatography (1\% EtOAc/Toluene, $30 \mathrm{~g} \mathrm{SiO}_{2}$ ) gave the product $(82 \mathrm{mg}, 0.22 \mathrm{mmol}, 55 \%)$, which was further purified by Kugelrohr distillation $\left(250{ }^{\circ} \mathrm{C}, 0.1\right.$ torr) to give the pure lactone $(72 \mathrm{mg}$, $0.194 \mathrm{mmol}, 48 \%)$.

TLC (1\% EtOAc/Toluene, $\mathrm{KMnO}_{4}$ ): 0.42; $\left.{ }^{1} \mathrm{H} \mathrm{NMR} \mathrm{(400} \mathrm{MHz,} \mathrm{CDCl}_{3}\right)$ : $7.76(\mathrm{~m}, 2 \mathrm{H})$, $7.27(\mathrm{~m}, 1 \mathrm{H}), 7.11$ (apparent t, $J=7.8 \mathrm{~Hz}, 1 \mathrm{H}) 6.32(\mathrm{~s}, 1 \mathrm{H}), 5.50(\mathrm{~s}, 1 \mathrm{H}), 5.06(\mathrm{~s}, 1 \mathrm{H}), 1.32$ (s, 3H), 1.15-0.90 (m, 5H), $0.78(\mathrm{~m}, 1 \mathrm{H}), 0.73(\mathrm{t}, J=7.0 \mathrm{~Hz}, 3 \mathrm{H}) ;{ }^{13} \mathrm{C}$ NMR $(100 \mathrm{MHz}$, $\left.\mathrm{CDCl}_{3}\right): \square 169.9,143.3,137.4,137.2$. 134.9, 130.0, 125.3, 121.4, 94.1, 87.3, 46.9, 34.3, 25.0, 22.8, 21.6, 13.8; IR $\left(\mathrm{CH}_{2} \mathrm{Cl}_{2}\right.$ cast, $\left.\mathrm{cm}^{-1}\right)$ : 2957, 1770, 1660, 1592, 1119; HRMS (EI, $m / z)$ Calcd for $\mathrm{C}_{16} \mathrm{H}_{19} \mathrm{O}_{2} \mathrm{I}: 370.04297$. Found: 370.04344.

\subsection{5 $\left(4 R^{\star}, 5 S^{*}\right)$-4-Butyl-4-methyl-3-methylene-5-pentafluorophenyl-dihydro-furan-2-one $2 r$}

A solution of allylboronate 1e $(132 \mathrm{mg}, 0.424 \mathrm{mmol})$ and pentafluorobenzaldehyde $(132 \mathrm{mg}, 0.674 \mathrm{mmol})$ in toluene $(0.4 \mathrm{~mL})$ was treated with $\mathrm{Sc}(\mathrm{OTf})_{3}(21 \mathrm{mg}, 0.043 \mathrm{mmol})$ and stirred at room temperature under Ar for $24 \mathrm{~h}$. Work up as described in Section 6.4.2 gave the product as a crude oil $(212 \mathrm{mg})$. Flash chromatography (1\% EtOAc/Toluene, $22 \mathrm{~g}$ $\mathrm{SiO}_{2}$ ) gave the product ( $75 \mathrm{mg}, 0.22 \mathrm{mmol}, 53 \%$ ), which was further purified by Kugelrohr distillation $\left(250^{\circ} \mathrm{C}, 0.1\right.$ torr) to give the pure lactone $(66 \mathrm{mg}, 0.198 \mathrm{mmol}, 47 \%)$.

TLC (1\% EtOAc/Toluene, $\mathrm{KMnO}_{4}$ ): 0.42; $\left.{ }^{1} \mathrm{H} \mathrm{NMR} \mathrm{(400} \mathrm{MHz,} \mathrm{CDCl}_{3}\right)$ : $\square 6.34$ (s, 1H), $5.57(\mathrm{~s}, 1 \mathrm{H}), 5.48(\mathrm{~s}, 1 \mathrm{H}), 1.55(\mathrm{dt}, J=13.3 \mathrm{~Hz}, 4.2 \mathrm{~Hz}, 1 \mathrm{H}), 1.35(\mathrm{~s}, 3 \mathrm{H}), 1.35-0.90(\mathrm{~m}$, 
$5 \mathrm{H}), 0.75(\mathrm{t}, J=7.2 \mathrm{~Hz}, 3 \mathrm{H}) ;{ }^{13} \mathrm{C} \mathrm{NMR}\left(100 \mathrm{MHz}, \mathrm{CDCl}_{3}\right): \square 169.3,146.3$ and $143.8,{ }^{22}$ 142.7, 142.6 and $140.1,{ }^{22} 139.0$ and $136.5,{ }^{22} 121.6,110.3,{ }^{22} 80.6,46.2,34.5,25.5,24.6$, 22.9, 13.7; ${ }^{19} \mathrm{~F}$ NMR (376 MHz, $\mathrm{CDCl}_{3}$ ): $\square-139.6$ (br s, 2F), -152.3 (tt, $J=20.9 \mathrm{~Hz}$, $2.6 \mathrm{~Hz}, 1 \mathrm{~F}),-161.1(\mathrm{dt}, J=22.1 \mathrm{~Hz}, 8.0 \mathrm{~Hz}, 2 \mathrm{~F}) ; \mathrm{IR}\left(\mathrm{CH}_{2} \mathrm{Cl}_{2}\right.$ cast, $\left.\mathrm{cm}^{-1}\right): 2960,1781,1655$, 1130; HRMS (ES, $m / z$ ) Calcd for $\mathrm{C}_{16} \mathrm{H}_{15} \mathrm{~F}_{5} \mathrm{O}_{2} \mathrm{Na}$ : 357.088991. Found: 357.088836; Anal. Calcd for $\mathrm{C}_{16} \mathrm{H}_{15} \mathrm{~F}_{5} \mathrm{O}_{2}$ : C, 57.48; H, 4.53. Found: C, 57.40; H, 4.42.

\subsection{6 (4R $\left.R^{\star}, 5 R^{\star}\right)$-4-Butyl-4-methyl-3-methylene-5-(4-nitrophenyl)-dihydro-furan-2-one 2s}

A solution of allylboronate 1e (132 mg, $0.424 \mathrm{mmol})$ and 4-nitrobenzaldehyde (72 mg, $0.47 \mathrm{mmol})$ in toluene $(0.5 \mathrm{~mL})$ was treated with $\mathrm{Sc}(\mathrm{OTf})_{3}(22 \mathrm{mg}, 0.046 \mathrm{mmol})$ and stirred at room temperature under Ar for $24 \mathrm{~h}$. Work up as described in Section 6.4.2 gave the product as a crude oil $(154 \mathrm{mg})$. Flash chromatography (1\% EtOAc/Toluene, $16 \mathrm{~g}$ $\mathrm{SiO}_{2}$ ) gave the pure product $(71 \mathrm{mg}, 0.25 \mathrm{mmol}, 58 \%)$. The spectroscopic data for this compound were identical to that described in Section 5.1.5 (page 19).

\subsection{7 $\left(4 R^{*}, 5 S^{\star}\right)$-4-Butyl-4-methyl-3-methylene-5-(1-naphthyl)-dihydro-furan-2-one $2 \mathrm{t}$}

A solution of allylboronate 1e (124 mg, $0.399 \mathrm{mmol})$ and 1-naphthaldehyde $(56 \mathrm{mg}$, $0.36 \mathrm{mmol})$ in toluene $(0.4 \mathrm{~mL})$ was treated with $\mathrm{Sc}(\mathrm{OTf})_{3}(14 \mathrm{mg}, 0.28 \mathrm{mmol})$ and stirred at room temperature under Ar for $24 \mathrm{~h}$. Work up as described in Section 6.4.2 gave the product as a crude oil $(105 \mathrm{mg})$. Flash chromatography $\left(25 \% \mathrm{Et}_{2} \mathrm{O} / \mathrm{Hexanes}, 9 \mathrm{~g} \mathrm{SiO}_{2}\right)$ gave the pure product ( $46 \mathrm{mg}, 0.16 \mathrm{mmol}, 44 \%$ ).

TLC (25\% Et 2 O/Hexanes, UV/KMnO $\left.{ }_{4}\right): 0.24 ;{ }^{1} \mathrm{H} \mathrm{NMR}\left(500 \mathrm{MHz}, \mathrm{CDCl}_{3}\right): \square 7.88(\mathrm{~m}$, 1H), $7.85(\mathrm{~m}, 1 \mathrm{H}), 7.83(\mathrm{~m}, 1 \mathrm{H}), 7.61(\mathrm{~m}, 1 \mathrm{H}), 7.45(\mathrm{~m}, 3 \mathrm{H}), 6.37(\mathrm{~s}, 1 \mathrm{H}), 6.08(\mathrm{~s}, 1 \mathrm{H})$, $5.53(\mathrm{~s}, 1 \mathrm{H}), 1.43(\mathrm{~s}, 3 \mathrm{H}), 1.22(\mathrm{~m}, 1 \mathrm{H}), 0.94(\mathrm{~m}, 3 \mathrm{H}), 0.76(\mathrm{~m}, 2 \mathrm{H}), 0.61 \quad(\mathrm{t}, J=7.2 \mathrm{~Hz}$, $3 \mathrm{H}) ;{ }^{13} \mathrm{C}$ NMR (125 MHz, $\mathrm{CDCl}_{3}$ ): $\square$ 170.6, 144.2, 133.7, 130.9, 130.7, 129.1, 128.9, 126.2, 125.6, 125.3, 125.1, 123.0, 121.2, 84.9, 47.6, 34.8, 25.1, 22.8, 22.7, 13.7; Anal. Calcd for $\mathrm{C}_{20} \mathrm{H}_{22} \mathrm{O}_{2}: \mathrm{C}, 81.58 ; \mathrm{H}, 7.55$. Found: $\mathrm{C}, 81.23 ; \mathrm{H}, 7.57$.

(22) These signals are complex, broad patterns due to ${ }^{13} \mathrm{C}-{ }^{19} \mathrm{~F}$ splitting. They are well resolved singlets at $145.1,141.4,137.7$ and $110.2 \mathrm{ppm}$ respectively in ${ }^{19} \mathrm{~F}$ decoupled spectra. 


\subsection{8 $\left(4 R^{\star}, 5 R^{\star}\right)$-4-Butyl-4-methyl-3-methylene-5-(4-tolyl)-dihydro-furan-2-one $2 u$}

A solution of allylboronate $1 \mathrm{e}(118 \mathrm{mg}, 0.424 \mathrm{mmol})$ and $p$-tolualdehyde $(66 \mathrm{mg}$, $0.55 \mathrm{mmol})$ in toluene $(0.4 \mathrm{~mL})$ was treated with $\mathrm{Sc}(\mathrm{OTf})_{3}(18 \mathrm{mg}, 0.037 \mathrm{mmol})$ and stirred at room temperature under Ar for $19 \mathrm{~h}$. Work up as described in Section 6.4.2 gave the product as a crude oil $(130 \mathrm{mg})$. Flash chromatography (1\% EtOAc/Toluene, $15 \mathrm{~g} \mathrm{SiO}_{2}$ ) yielded the pure product $(73 \mathrm{mg}, 0.28 \mathrm{mmol}, 75 \%)$.

TLC (1\% EtOAc/Toluene, UV/KMnO ${ }_{4}$ ): 0.41; ${ }^{1} \mathrm{H}$ NMR (400 MHz, $\mathrm{CDCl}_{3}$ ): 7.17 (s, $5 \mathrm{H}), 6.30(\mathrm{~s}, 1 \mathrm{H}), 5.47(\mathrm{~s}, 1 \mathrm{H}), 5.10(\mathrm{~s}, 1 \mathrm{H}), 2.35(\mathrm{~s}, 3 \mathrm{H}), 1.30(\mathrm{~s}, 3 \mathrm{H}), 1.02(\mathrm{~m}, 5 \mathrm{H}), 0.80$ $(\mathrm{m}, 1 \mathrm{H}), 0.71(\mathrm{t}, J=6.9 \mathrm{~Hz}, 3 \mathrm{H}) ;{ }^{13} \mathrm{C} \mathrm{NMR}\left(100 \mathrm{MHz}, \mathrm{CDCl}_{3}\right)$ : $\mathrm{Q} 170.5,144.0,138.0$, 131.8, 128.9, 126.0, 120.8, 88.6, 46.9, 34.1, 25.1, 22.8, 21.4, 21.1, 13.8; HRMS (EI, $m / z$ ) Calcd for $\mathrm{C}_{17} \mathrm{H}_{22} \mathrm{O}_{2}: 258.16199$. Found: 258.16159; Anal. Calcd for $\mathrm{C}_{17} \mathrm{H}_{22} \mathrm{O}_{2}: \mathrm{C}, 79.02$; H, 8.60. Found: C, 78.18; H, 8.74.

\subsection{9 $\left(4 R^{\star}, 5 S^{\star}\right)$-4-Ethyl-5-(2-methoxyphenyl)-4-methyl-3-methylene-dihydro-furan-2-one 2v}

A solution of allylboronate $1 \mathrm{c}(97 \mathrm{mg}, 0.34 \mathrm{mmol})$ and $o$-anisaldehyde $(44 \mathrm{mg}$, $0.32 \mathrm{mmol})$ in toluene $(0.4 \mathrm{~mL})$ was treated with $\mathrm{Sc}(\mathrm{OTf})_{3}(14 \mathrm{mg}, 0.030 \mathrm{mmol})$ and stirred at room temperature under $\mathrm{Ar}$ for $24 \mathrm{~h}$. Work up as described in Section 6.4.2 gave the product as a crude oil $(66 \mathrm{mg}) .{ }^{1} \mathrm{H} \mathrm{NMR}\left(500 \mathrm{MHz}, \mathrm{CDCl}_{3}\right)$ showed that the product was a 2:1 mixture of diastereomers.

\subsubsection{0 $\left(4 R^{\star}, 5 S^{\star}\right)-4-E$ thyl-4-methyl-3-methylene-5-[Z-2-(4-nitrophenyl)-ethenyl]-dihydro-furan-2- one $2 w$}

A solution of allylboronate $1 \mathrm{c}(82 \mathrm{mg}, 0.29 \mathrm{mmol})$ and 4-nitrocinnamaldehyde $(52 \mathrm{mg}, 0.30 \mathrm{mmol})$ in toluene $(0.3 \mathrm{~mL})$ was treated with $\mathrm{Sc}(\mathrm{OTf})_{3}(15 \mathrm{mg}, 0.030 \mathrm{mmol})$ and stirred at room temperature under Ar for $24 \mathrm{~h}$. Work up as described in Section 6.4.2 gave the product as a crude oil $(103 \mathrm{mg}) .{ }^{1} \mathrm{H}$ NMR $\left(500 \mathrm{MHz}, \mathrm{CDCl}_{3}\right)$ showed that the product was a 2.8:1 mixture of diastereomers favouring the expected isomer.

A diastereomerically pure sample was prepared by refluxing a solution of allylboronate 1c (103 mg, $0.364 \mathrm{mmol})$ and 4-nitrocinnamaldehyde $(51 \mathrm{mg}, 0.29 \mathrm{mmol})$ in toluene $(0.7 \mathrm{~mL})$ under Ar for $18 \mathrm{~h}$. Concentration of the mixture (125 mg crude) followed by flash chromatography ( $25 \%$ EtOAc/Hexanes, $7 \mathrm{~g}$ silica) yielded the pure lactone $(68 \mathrm{mg}$, $0.23 \mathrm{mmol}, 82 \%)$. 
TLC (25\% EtOAc/Hexanes, UV/ $\mathrm{KMnO}_{4}$ ): 0.32; ${ }^{1} \mathrm{H} \mathrm{NMR}\left(400 \mathrm{MHz}, \mathrm{CDCl}_{3}\right): \square 8.18(\mathrm{AB}$, $J=8.8 \mathrm{~Hz}, 2 \mathrm{H}), 7.50(\mathrm{AB}, J=8.8 \mathrm{~Hz}, 2 \mathrm{H}), 6.71(\mathrm{~d}, J=15.8 \mathrm{~Hz}, 1 \mathrm{H}), 6.32(\mathrm{~s}, 1 \mathrm{H}), 6.25$ (dd, $J=16.0 \mathrm{~Hz}, 6.7 \mathrm{~Hz}, 1 \mathrm{H}), 5.49(\mathrm{~s}, 1 \mathrm{H}), 5.28(\mathrm{~s}, 1 \mathrm{H}), 4.84(\mathrm{dd}, J=6.7 \mathrm{~Hz}, 1.2 \mathrm{~Hz}, 1 \mathrm{H}$ ), $1.67(\mathrm{~m}, 2 \mathrm{H}), 1.12(\mathrm{~s}, 3 \mathrm{H}), 0.94(\mathrm{t}, J=7.5 \mathrm{~Hz}, 3 \mathrm{H}) ;{ }^{13} \mathrm{C} \mathrm{NMR}\left(125 \mathrm{MHz}, \mathrm{CDCl}_{3}\right)$ : $\square$ 169.9, 147.4, 142.8, 142.1, 131.0, 129.1, 127.3, 124.0, 121.7, 84.7, 46.5, 32.8, 21.4, 8.4; IR $\left(\mathrm{CH}_{2} \mathrm{Cl}_{2}\right.$ cast, $\left.\mathrm{cm}^{-1}\right): 2969,1764,1655,1596,1517,1343,1108,971,815$; HRMS (EI, $m / z)$ Calcd for $\mathrm{C}_{16} \mathrm{H}_{19} \mathrm{NO}_{4}$ : 289.13141. Found: 289.13096; Anal. Calcd for $\mathrm{C}_{16} \mathrm{H}_{19} \mathrm{NO}_{4}$ : C, 66.88; H, 5.98; N, 4.88. Found: C, 66.82; H, 5.92; N, 4.74.

\subsubsection{1 $\left(4 R^{\star}, 5 R^{\star}\right)$-4-Butyl-5-(4-methoxyphenyl)-4-methyl-3-methylene-dihydro-furan-2-one $2 \mathrm{x}$}

A solution of allylboronate $1 \mathrm{e}(135 \mathrm{mg}, 0.434 \mathrm{mmol})$ and $p$-anisaldehyde $(90 \mathrm{mg}$, $0.66 \mathrm{mmol})$ in toluene $(0.9 \mathrm{~mL})$ was treated with $\mathrm{Sc}(\mathrm{OTf})_{3}(22 \mathrm{mg}, 0.044 \mathrm{mmol})$ and stirred at room temperature under Ar for $16 \mathrm{~h}$. Work up as described in Section 6.4.2 gave the product as a crude oil $(190 \mathrm{mg}) .{ }^{1} \mathrm{H} \mathrm{NMR}\left(500 \mathrm{MHz}, \mathrm{CDCl}_{3}\right)$ showed that the product was a 1.8:1 mixture of diastereomers favouring the expected isomer.

A diastereomerically pure sample of $\mathbf{2} \mathbf{x}$ was prepared by refluxing a solution of allylboronate 1e $(112 \mathrm{mg}, 0.360 \mathrm{mmol})$ and $p$-anisaldehyde $(45 \mathrm{mg}, 0.33 \mathrm{mmol})$ in toluene $(1 \mathrm{~mL})$ under $\mathrm{Ar}$ for $24 \mathrm{~h}$. Concentration of the mixture followed by flash chromatography (25\% $\mathrm{Et}_{2} \mathrm{O} / \mathrm{Hexanes,} 11 \mathrm{~g}$ silica) yielded the pure lactone (56 mg, $\left.0.20 \mathrm{mmol}, 64 \%\right)$.

${ }^{1} \mathrm{H}$ NMR (400 MHz, $\left.\mathrm{CDCl}_{3}\right): \square 7.19(\mathrm{AB}, J=8.9 \mathrm{~Hz}, 2 \mathrm{H}), 6.89(\mathrm{AB}, J=8.7 \mathrm{~Hz}, 2 \mathrm{H}), 6.29$ (s, 1H), $5.44(\mathrm{~s}, 1 \mathrm{H}), 5.08(\mathrm{~s}, 1 \mathrm{H}), 3.81(\mathrm{~s}, 3 \mathrm{H}), 1.28(\mathrm{~s}, 3 \mathrm{H}), 1.15-0.90(\mathrm{~m}, 5 \mathrm{H}), 0.79(\mathrm{~m}$, $1 \mathrm{H}), 0.71(\mathrm{t}, J=6.7 \mathrm{~Hz}, 3 \mathrm{H}) ;{ }^{13} \mathrm{C} \mathrm{NMR}\left(125 \mathrm{MHz}, \mathrm{CDCl}_{3}\right)$ : $\square 170.3,159.5,144.0,127.4$, $126.8,120.8,113.6,88.5,55.3,47.0,34.2,25.2,23.0,21.6,13.9 ;$ IR $\left(\mathrm{CH}_{2} \mathrm{Cl}_{2}\right.$ cast, $\left.\mathrm{cm}^{-1}\right)$ : 2958, 1769, 1659, 1613, 1251, 817; HRMS (EI, $m / z$ ) Calcd for $\mathrm{C}_{17} \mathrm{H}_{22} \mathrm{O}_{3}$ : 274.15689. Found: 274.15514.

\subsubsection{2 $\left(4 R^{\star}, 5 R^{\star}\right)$-4-Butyl-4-methyl-3-methylene-5-(E-1-propenyl)-dihydro-furan-2-one 2y}

A solution of allylboronate $1 \mathrm{e}(129 \mathrm{mg}, 0.416 \mathrm{mmol})$ and crotonaldehyde $(43 \mathrm{mg}$, $0.61 \mathrm{mmol})$ in toluene $(0.8 \mathrm{~mL})$ was treated with $\mathrm{Sc}(\mathrm{OTf})_{3}(21 \mathrm{mg}, 0.044 \mathrm{mmol})$ and stirred at room temperature under Ar for $20 \mathrm{~h}$. Work up as described in Section 6.4.2 gave the crude product as a 1.6:1 mixture of diastereomers $(137 \mathrm{mg})$, which could be separated by flash chromatography ( $5 \% \mathrm{Et}_{2} \mathrm{O} /$ Hexanes, $10 \mathrm{~g}$ silica).

Major Isomer: (4R*, 5R*)-2y, $32 \mathrm{mg}, 0.15 \mathrm{mmol}, 36 \%$. ${ }^{1} \mathrm{H}$ NMR $\left(500 \mathrm{MHz}, \mathrm{CDCl}_{3}\right)$ : $\square 6.19(\mathrm{~s}, 1 \mathrm{H}), 5.82(\mathrm{~m}, 1 \mathrm{H}), 5.49(\mathrm{~m}, 1 \mathrm{H}), 5.39(\mathrm{~s}, 1 \mathrm{H}), 4.38(\mathrm{~d}, J=8.1 \mathrm{~Hz}, 1 \mathrm{H}), 1.76(\mathrm{~m}$, 
$3 \mathrm{H}), 1.4-1.1(\mathrm{~m}, 6 \mathrm{H}), 1.13(\mathrm{~s}, 3 \mathrm{H}), 0.84(\mathrm{t}, J=7.1 \mathrm{~Hz}, 3 \mathrm{H}) ;{ }^{13} \mathrm{C}$ NMR $\left(125 \mathrm{MHz}, \mathrm{CDCl}_{3}\right)$ : प170.2, 144.0, 132.5, 124.0, 120.2, 88.5, 46.1, 34.7, 25.6, 23.1, 21.7, 18.0, 14.0; IR $\left(\mathrm{CH}_{2} \mathrm{Cl}_{2}\right.$ cast, $\mathrm{cm}^{-1}$ ): 2959, 1766, 1661, 1106, 968; HRMS (EI, $m / z$ ) Calcd for $\mathrm{C}_{13} \mathrm{H}_{20} \mathrm{O}_{2}: 208.14633$. Found: 208.14593; Anal. Calcd for $\mathrm{C}_{13} \mathrm{H}_{20} \mathrm{O}_{2}$ : C, 74.94; H, 9.70. Found: C, 74.85; H, 9.57.

Minor Isomer: $\left(4 R^{*}, 5 S^{*}\right)-\mathbf{2 y}, 27 \mathrm{mg}, 0.099 \mathrm{mmol}, 23 \% .{ }^{1} \mathrm{H}$ NMR $\left(500 \mathrm{MHz}, \mathrm{CDCl}_{3}\right)$ : $\square 6.21(\mathrm{~s}, 1 \mathrm{H}), 5.77(\mathrm{~m}, 1 \mathrm{H}), 5.40(\mathrm{~s}, 1 \mathrm{H}), 5.36(\mathrm{~m}, 1 \mathrm{H}), 4.56(\mathrm{~d}, J=8.2 \mathrm{~Hz}, 1 \mathrm{H}), 1.72(\mathrm{~m}$, $3 \mathrm{H}), 1.48(\mathrm{~m}, 2 \mathrm{H}), 1.30-1.15(\mathrm{~m}, 4 \mathrm{H}), 1.04(\mathrm{~s}, 3 \mathrm{H}), 0.87(\mathrm{t}, J=7.0 \mathrm{~Hz}, 3 \mathrm{H}) ;{ }^{13} \mathrm{C}$ NMR (125 MHz, $\left.\mathrm{CDCl}_{3}\right)$ : $\square$ 170.2, 144.0, 131.4, 126.0, 120.4, 86.5, 45.5, 40.0, 26.1, 23.2, 21.8, 17.9, 14.0; IR $\left(\mathrm{CH}_{2} \mathrm{Cl}_{2}\right.$ cast, $\left.\mathrm{cm}^{-1}\right): 2960,1766,1661,1105,966$; HRMS (EI, $\left.m / z\right)$ Calcd for $\mathrm{C}_{13} \mathrm{H}_{20} \mathrm{O}_{2}:$ 208.14633. Found: 208.14616; Anal. Calcd for $\mathrm{C}_{13} \mathrm{H}_{20} \mathrm{O}_{2}$ : C, 74.94; H, 9.70. Found: C, 74.97; H, 9.89.

\subsubsection{3 $\left(4 R^{\star}, 5 S^{\star}\right)$-3-Butyl-3-ethyl-4-methylene-3,4-dihydro-2H-[2,2']bifuranyl-5-one $2 z$}

A solution of allylboronate 1i $(130 \mathrm{mg}, 0.402 \mathrm{mmol})$ and furfural $(38 \mathrm{mg}$, $0.40 \mathrm{mmol})$ in toluene $(0.4 \mathrm{~mL})$ was treated with $\mathrm{Sc}(\mathrm{OTf})_{3}(20 \mathrm{mg}, 0.04 \mathrm{mmol})$ and stirred at room temperature under $\mathrm{Ar}$ for $24 \mathrm{~h}$. Work up as described in Section 6.4.2 gave the product as a crude oil $(116 \mathrm{mg}) .{ }^{1} \mathrm{H} \mathrm{NMR}\left(500 \mathrm{MHz}, \mathrm{CDCl}_{3}\right)$ showed that the product was a 2.5:1 mixture of diastereomers favouring the expected isomer.

A diastereomerically pure sample of $\mathbf{2 z}$ was prepared by refluxing a solution of allylboronate $1 \mathbf{i}(136 \mathrm{mg}, 0.419 \mathrm{mmol})$ and furfural $(40 \mathrm{mg}, 0.42 \mathrm{mmol})$ in toluene $(0.8 \mathrm{~mL})$ under Ar for $24 \mathrm{~h}$. Concentration of the mixture followed by flash chromatography (2.5\% EtOAc/Toluene, $26 \mathrm{~g}$ silica) yielded the pure lactone $(78 \mathrm{mg}$, $0.31 \mathrm{mmol}, 74 \%)$.

${ }^{1} \mathrm{H}$ NMR (500 MHz, CDCl $)$ : $77.36(\mathrm{~m}, 1 \mathrm{H}), 6.35(\mathrm{~s}, 1 \mathrm{H}), 6.33(\mathrm{~m}, 1 \mathrm{H}), 6.31(\mathrm{~m}, 1 \mathrm{H})$, $5.42(\mathrm{~s}, 1 \mathrm{H}), 5.18(\mathrm{~s}, 1 \mathrm{H}), 1.71(\mathrm{~m}, 2 \mathrm{H}), 1.48(\mathrm{~m}, 1 \mathrm{H}), 1.24-0.96(\mathrm{~m}, 5 \mathrm{H}), 0.85(\mathrm{t}$, $J=7.5 \mathrm{~Hz}, 3 \mathrm{H}), 0.74(\mathrm{t}, J=7.1 \mathrm{~Hz}, 3 \mathrm{H}) ;{ }^{13} \mathrm{C} \mathrm{NMR}\left(125 \mathrm{MHz}, \mathrm{CDCl}_{3}\right): \square$ 170.2, 149.9, $142.8,141.5,121.5,110.3,109.4,81.3,50.0,31.6,28.8,25.3,23.0,13.7$, 7.6; Anal. Calcd for $\mathrm{C}_{15} \mathrm{H}_{20} \mathrm{O}_{3}:$ C, 72.54; H, 8.13. Found: C, 72.45; H, 8.41.

\subsubsection{4 $\left(4 R^{\star}, 5 R^{\star}\right)$-4-Butyl-4-ethyl-3-methylene-5-(4-methylthiophenyl)-dihydro-furan-2-one 2aa}

A solution of allylboronate $1 \mathbf{i}(137 \mathrm{mg}, 0.421 \mathrm{mmol})$ and 4-methylthiobenzaldehyde $(65 \mathrm{mg}, 0.43 \mathrm{mmol})$ in toluene $(0.4 \mathrm{~mL})$ was treated with $\mathrm{Sc}(\mathrm{OTf})_{3}(21 \mathrm{mg}, 0.043 \mathrm{mmol})$ and stirred at room temperature under Ar for $7 \mathrm{~d}$. Work up as described in Section 6.4.2 
gave the product as a crude oil $(135 \mathrm{mg}) .{ }^{1} \mathrm{H}$ NMR $\left(500 \mathrm{MHz}, \mathrm{CDCl}_{3}\right)$ showed that the product was a 2.5:1 mixture of diastereomers.

\subsection{Catalysed allylborations with aliphatic aldehydes}

\subsubsection{Lewis acid catalysed reactions with excess aldehyde}

\subsubsection{1 $\left(4 R^{*}, 5 S^{\star}\right)-4$-Ethyl-4-methyl-3-methylene-5-phenethyl-dihydro-furan-2-one 2ab}

A solution of allylboronate 1c $(103 \mathrm{mg}, 0.365 \mathrm{mmol})$ and hydrocinnamaldehyde $(74 \mathrm{mg}, 0.55 \mathrm{mmol})$ in toluene $(0.6 \mathrm{~mL})$ was treated with $\mathrm{Sc}(\mathrm{OTf})_{3}(20 \mathrm{mg}, 0.041 \mathrm{mmol})$ and stirred at room temperature under Ar for $24 \mathrm{~h}$. Reaction was then diluted with water $(5 \mathrm{~mL})$ and extracted with $\mathrm{Et}_{2} \mathrm{O}(3 \times 5 \mathrm{~mL})$. The combined extracts were washed with brine $(5 \mathrm{~mL})$, dried $\left(\mathrm{Na}_{2} \mathrm{SO}_{4}\right)$ and concentrated to give the crude product $(145 \mathrm{mg})$. Flash chromatography (10\% $\mathrm{Et}_{2} \mathrm{O} / \mathrm{Hexanes}, 7 \mathrm{~g} \mathrm{SiO}_{2}$ ) gave the pure product $(57 \mathrm{mg}, 0.23 \mathrm{mmol}$, $64 \%)$. Similarly, use of $\mathrm{Cu}(\mathrm{OTf})_{2}$ or $\mathrm{Yb}(\mathrm{OTf})_{3}$ instead of scandium triflate afforded the lactone product in $63 \%$ and $66 \%$ yield respectively.

TLC (1\% EtOAc/Toluene, $\mathrm{KMnO}_{4}$ ): 0.26; $\left.{ }^{1} \mathrm{H} \mathrm{NMR} \mathrm{(500} \mathrm{MHz,} \mathrm{CDCl}_{3}\right)$ : $7.28(\mathrm{~m}, 2 \mathrm{H})$, $7.18(\mathrm{~m}, 3 \mathrm{H}), 6.23(\mathrm{~s}, 1 \mathrm{H}), 5.41(\mathrm{~s}, 1 \mathrm{H}), 4.17(\mathrm{dd}, J=10.8 \mathrm{~Hz}, 2.9 \mathrm{~Hz}), 2.94(\mathrm{~m}, 1 \mathrm{H}), 2.68$ (m, 1H), $1.78(\mathrm{~m}, 2 \mathrm{H}), 1.52(\mathrm{~m}, 2 \mathrm{H}), 1.08(\mathrm{~s}, 3 \mathrm{H}), 0.80(\mathrm{t}, J=7.4 \mathrm{~Hz}, 3 \mathrm{H}) ;{ }^{13} \mathrm{C}$ NMR (125 MHz, $\mathrm{CDCl}_{3}$ ): $\square$ 170.4, 144.0, 141.0, 128.5, 126.1, 120.6, 84.4, 45.0, 33.6, 32.8, 32.3, 20.2, 8.3; IR $\left(\mathrm{CH}_{2} \mathrm{Cl}_{2}\right.$ cast, $\left.\mathrm{cm}^{-1}\right): 3061,2967,1762,1659,1602,1113,751,700$; HRMS (EI, $m / z$ ) Calcd for $\mathrm{C}_{16} \mathrm{H}_{22} \mathrm{O}_{2}$ : 244.14633. Found: 244.14648; Anal. Calcd for $\mathrm{C}_{16} \mathrm{H}_{22} \mathrm{O}_{2}$ : C, 78.64; H, 8.27. Found: C, 78.28; H, 8.38.

\subsubsection{2 $\left(4 R^{*}, 5 R^{*}\right)$-5-Benzyloxymethyl-4-ethyl-4-methyl-3-methylene-dihydro-furan-2-one 2ac}

A solution of allylboronate 1c $(93 \mathrm{mg}, 0.33 \mathrm{mmol})$ and benzyloxyacetaldehyde (74 mg, $0.49 \mathrm{mmol})$ in toluene $(0.7 \mathrm{~mL})$ was treated with $\mathrm{Sc}(\mathrm{OTf})_{3}(19 \mathrm{mg}, 0.031 \mathrm{mmol})$ and stirred at room temperature under Ar for $24 \mathrm{~h}$. Reaction was diluted with water $(5 \mathrm{~mL})$ and extracted with $\mathrm{Et}_{2} \mathrm{O}(3 \times 5 \mathrm{~mL})$. The combined extracts were washed with brine $(5 \mathrm{~mL})$, dried $\left(\mathrm{Na}_{2} \mathrm{SO}_{4}\right)$ and concentrated to give the crude product $(98 \mathrm{mg})$. Flash chromatography (25\% $\mathrm{Et}_{2} \mathrm{O} / \mathrm{Hexanes}, 10 \mathrm{~g} \mathrm{SiO}_{2}$ ) followed by Kugelrohr distillation ( $250{ }^{\circ} \mathrm{C}, 0.1$ torr) gave the pure product (44 $\mathrm{mg}, 0.17 \mathrm{mmol}, 52 \%$ ).

$\operatorname{TLC}\left(25 \% \mathrm{Et}_{2} \mathrm{O} / \mathrm{Hexanes} \mathrm{KMnO}_{4}\right): 0.19 ;{ }^{1} \mathrm{H} \mathrm{NMR}\left(500 \mathrm{MHz}, \mathrm{CDCl}_{3}\right)$ : $7.32(\mathrm{~m}, 2 \mathrm{H})$, $7.26(\mathrm{~m}, 3 \mathrm{H}), 6.19(\mathrm{~s}, 1 \mathrm{H}), 5.37(\mathrm{~s}, 1 \mathrm{H}), 4.52(\mathrm{~s}, 2 \mathrm{H}), 4.32(\mathrm{t}, J=4.5 \mathrm{~Hz}, 1 \mathrm{H}), 3.60(\mathrm{dq}$, 
$J=10.8 \mathrm{~Hz}, 4.3 \mathrm{~Hz}, 2 \mathrm{H}), 1.55(\mathrm{~m}, 2 \mathrm{H}), 1.18(\mathrm{~s}, 3 \mathrm{H}), 0.85(\mathrm{t}, J=7.5 \mathrm{~Hz}, 3 \mathrm{H})$; IR $\left(\mathrm{CH}_{2} \mathrm{Cl}_{2}\right.$ cast, $\left.\mathrm{cm}^{-1}\right): 3030,2968,1764,1657,1105,737,697$; HRMS (EI, $\left.m / z\right)$ Calcd for $\mathrm{C}_{16} \mathrm{H}_{20} \mathrm{O}_{3}$ : 260.14124. Found: 260.14190; Anal. Calcd for $\mathrm{C}_{16} \mathrm{H}_{20} \mathrm{O}_{3}: \mathrm{C}, 73.80 ; \mathrm{H}, 7.76$. Found: C, 73.46; H, 7.96 .

\subsubsection{3 $\left(4 R^{*}, 5 S^{\star}\right)$-4-Ethyl-4-methyl-3-methylene-5-(2-methylpropyl) -dihydro-furan-2-one 2ad}

A solution of allylboronate 1c (84 mg, $0.30 \mathrm{mmol})$ and isovaleraldehyde $(24 \mathrm{mg}$, $0.28 \mathrm{mmol})$ in toluene $(0.6 \mathrm{~mL})$ was treated with $\mathrm{Sc}(\mathrm{OTf})_{3}(15 \mathrm{mg}, 0.030 \mathrm{mmol})$ and stirred at room temperature under $\mathrm{Ar}$ for $12 \mathrm{~h}$. Reaction was diluted with water $(5 \mathrm{~mL})$ and extracted with $\mathrm{Et}_{2} \mathrm{O}(3 \times 5 \mathrm{~mL})$. The combined extracts were washed with brine $(5 \mathrm{~mL})$, dried $\left(\mathrm{Na}_{2} \mathrm{SO}_{4}\right)$ and concentrated to give the crude product $(79 \mathrm{mg})$. Flash chromatography (50\% $\mathrm{CH}_{2} \mathrm{Cl}_{2} / \mathrm{Hexanes,} 4 \mathrm{~g} \mathrm{SiO}_{2}$ ) gave the pure product ( $25 \mathrm{mg}, 0.13 \mathrm{mmol}, 46 \%$ ).

${ }^{1} \mathrm{H} \mathrm{NMR}\left(500 \mathrm{MHz}, \mathrm{CDCl}_{3}\right)$ : $\square 6.21(\mathrm{~s}, 1 \mathrm{H}), 5.40(\mathrm{~s}, 1 \mathrm{H}), 4.26(\mathrm{dd}, J=11.4 \mathrm{~Hz}, 2.4 \mathrm{~Hz}$, 1H), $1.88(\mathrm{~m}, 1 \mathrm{H}), 1.55(\mathrm{~m}, 2 \mathrm{H}), 1.44$ (ddd, $J=14.2 \mathrm{~Hz}, 11.4 \mathrm{~Hz}, 4.1 \mathrm{~Hz}, 1 \mathrm{H}), 1.21$ (ddd, $J=14.2 \mathrm{~Hz}, 9.7 \mathrm{~Hz}, 2.4 \mathrm{~Hz}, 1 \mathrm{H}), 1.06$ (s, 3H), $0.94(\mathrm{dd}, J=6.7 \mathrm{~Hz}, 1.4 \mathrm{~Hz}, 6 \mathrm{H}), 0.86(\mathrm{t}$, $J=7.5 \mathrm{~Hz}, 3 \mathrm{H})$.

\subsubsection{4 $\left(4 R^{\star}, 5 S^{\star}\right)$-5-Cyclohexyl-4-ethyl-4-methyl-3-methylene-dihydro-furan-2-one 2ae}

A solution of allylboronate 1c $(126 \mathrm{mg}, 0.405 \mathrm{mmol}, 10: 1 \mathrm{Z}: E$ mixture $)$ and cyclohexanecarboxaldehyde $(111 \mathrm{mg}, 0.394 \mathrm{mmol})$ in toluene $(0.75 \mathrm{~mL})$ was treated with $\mathrm{Cu}(\mathrm{OTf})_{2}(15 \mathrm{mg}, 0.043 \mathrm{mmol})$ and stirred at $60{ }^{\circ} \mathrm{C}$ under Ar for $16 \mathrm{~h}$. Reaction was then brought to RT, diluted with water $(5 \mathrm{~mL})$ and extracted with $\mathrm{Et}_{2} \mathrm{O}(3 \times 5 \mathrm{~mL})$. The combined extracts were washed with brine $(5 \mathrm{~mL})$, dried $\left(\mathrm{Na}_{2} \mathrm{SO}_{4}\right)$ and concentrated to give the crude product $(164 \mathrm{mg})$. Flash chromatography $\left(1 \% \mathrm{EtOAc} / T o l u e n e, 10 \mathrm{~g} \mathrm{SiO}_{2}\right.$ ) gave the pure product ( $47 \mathrm{mg}, 0.21 \mathrm{mmol}, 54 \%$ ) as an inseparable 10:1 mixture of diastereomers. TLC (1\% EtOAc/Toluene, $\mathrm{KMnO}_{4}$ ): 0.20; ${ }^{1} \mathrm{H}$ NMR (500 MHz, $\mathrm{CDCl}_{3}$ ): $\square 6.16(\mathrm{~s}, 1 \mathrm{H})$, $5.33(\mathrm{~s}, 1 \mathrm{H}), 3.97(\mathrm{~d}, J=4.2 \mathrm{~Hz}, 1 \mathrm{H}), 1.80-1.45(\mathrm{~m}, 8 \mathrm{H}), 1.4-1.0(\mathrm{~m}, 5 \mathrm{H}), 1.17$ (s, 3H), $0.82(\mathrm{t}, J=7.4 \mathrm{~Hz}, 3 \mathrm{H}) ;{ }^{13} \mathrm{C}$ NMR (125 MHz, $\left.\mathrm{CDCl}_{3}\right)$ : $170.7,144.1,118.9,89.1,45.2$, 40.0, 34.4, 30.9, 27.4, 26.4, 26.1, 25.7, 19.9, 8.2; $\mathrm{IR}\left(\mathrm{CH}_{2} \mathrm{Cl}_{2}\right.$ cast, $\left.\mathrm{cm}^{-1}\right): 3093,2928,1765$, 1655, 1111; HRMS (EI, $m / z$ ) Calcd for $\mathrm{C}_{14} \mathrm{H}_{22} \mathrm{O}_{2}: 222.16199$. Found: 222.16219. 


\subsubsection{5 $\left(4 R^{\star}, 5 R^{\star}\right)$-4-Butyl-5-cyclohexyl-4-methyl-3-methylene-dihydro-furan-2-one 2af}

A solution of allylboronate 1 e $(109 \mathrm{mg}, 0.382 \mathrm{mmol})$ and cyclohexanecarboxaldehyde $(62 \mathrm{mg}, 0.55 \mathrm{mmol})$ in toluene $(0.8 \mathrm{~mL})$ was treated with $\mathrm{Sc}(\mathrm{OTf})_{3}(18 \mathrm{mg}, 0.037 \mathrm{mmol})$ and stirred at $60{ }^{\circ} \mathrm{C}$ under Ar for $24 \mathrm{~h}$. Reaction was brought to RT, diluted with water $(5 \mathrm{~mL})$ and extracted with $\mathrm{Et}_{2} \mathrm{O}(3 \times 5 \mathrm{~mL})$. The combined extracts were washed with brine $(5 \mathrm{~mL})$, dried $\left(\mathrm{Na}_{2} \mathrm{SO}_{4}\right)$ and concentrated to give the crude product $(182 \mathrm{mg})$. Flash chromatography $\left(5 \% \mathrm{Et}_{2} \mathrm{O} / \mathrm{Hexanes}, 10 \mathrm{~g} \mathrm{SiO}_{2}\right)$ gave the pure product ( $30 \mathrm{mg}, 0.12 \mathrm{mmol}, 32 \%$ ).

${ }^{1} \mathrm{H}$ NMR (500 MHz, $\mathrm{CDCl}_{3}$ ): $\square 6.16(\mathrm{~s}, 1 \mathrm{H}), 5.35$ (s, 1H), $3.71(\mathrm{~d}, J=8.9 \mathrm{~Hz}, 1 \mathrm{H}), 2.04$ $(\mathrm{m}, 1 \mathrm{H}), 1.74(\mathrm{~m}, 4 \mathrm{H}), 1.66(\mathrm{~m}, 1 \mathrm{H}), 1.42(\mathrm{~m}, 2 \mathrm{H}), 1.3-1.1(\mathrm{~m}, 7 \mathrm{H}), 1.21(\mathrm{~s}, 3 \mathrm{H}), 1.06(\mathrm{~m}$, $2 \mathrm{H}), 0.87(\mathrm{t}, J=7.1 \mathrm{~Hz}, 3 \mathrm{H}) ;{ }^{13} \mathrm{C} \mathrm{NMR}\left(125 \mathrm{MHz}, \mathrm{CDCl}_{3}\right): \square 170.5,145.0,119.5,91.6$, 45.3, 38.0, 33.1, 30.0, 29.6, 26.1, 25.6, 25.5, 25.4, 23.1, 23.0, 13.9; IR $\left(\mathrm{CH}_{2} \mathrm{Cl}_{2}\right.$ cast, $\left.\mathrm{cm}^{-1}\right)$ : 2930, 1766, 1659, 1111; HRMS (EI, $m / z$ ) Calcd for $\mathrm{C}_{16} \mathrm{H}_{26} \mathrm{O}_{2}:$ 250.19328. Found: 250.19308. Anal. Calcd for $\mathrm{C}_{16} \mathrm{H}_{26} \mathrm{O}_{2}$ : C, 76.74; H, 10.49. Found: C, 76.14; H, 10.37.

\subsubsection{6 $\left(4 R^{*}, 5 R^{\star}\right)$-4-Butyl -4-methyl-3-methylene-5-(2-propyl)-dihydro-furan-2-one 2ag}

A solution of allylboronate 1 e $(129 \mathrm{mg}, 0.415 \mathrm{mmol})$ and isobutyraldehyde $(52 \mathrm{mg}$, $0.72 \mathrm{mmol})$ in toluene $(0.8 \mathrm{~mL})$ was treated with $\mathrm{Sc}(\mathrm{OTf})_{3}(20 \mathrm{mg}, 0.041 \mathrm{mmol})$ and stirred at $60{ }^{\circ} \mathrm{C}$ under Ar for $18 \mathrm{~h}$. Reaction was brought to RT, diluted with water $(5 \mathrm{~mL})$ and extracted with $\mathrm{Et}_{2} \mathrm{O}(3 \times 5 \mathrm{~mL})$. The combined extracts were washed with brine $(5 \mathrm{~mL})$, dried $\left(\mathrm{Na}_{2} \mathrm{SO}_{4}\right)$ and concentrated to give the crude product $(202 \mathrm{mg})$. Flash chromatography $\left(5 \% \mathrm{Et}_{2} \mathrm{O} / \mathrm{Hexanes}, 10 \mathrm{~g} \mathrm{SiO}_{2}\right)$ gave the pure product $(28 \mathrm{mg}, 0.13 \mathrm{mmol}$, $32 \%)$.

${ }^{1} \mathrm{H}$ NMR (500 MHz, $\mathrm{CDCl}_{3}$ ): $\square 6.17$ (s, 1H), 5.36 (s, 1H), 3.65 (d, $\left.J=9.2 \mathrm{~Hz}, 1 \mathrm{H}\right), 2.04$ $(\mathrm{m}, 1 \mathrm{H}), 1.44(\mathrm{~m}, 2 \mathrm{H}), 1.3-1.1(\mathrm{~m}, 4 \mathrm{H}), 1.22(\mathrm{~s}, 3 \mathrm{H}), 1.06(\mathrm{~d}, J=6.5 \mathrm{~Hz}, 3 \mathrm{H}), 1.00$ (d, $J=6.6 \mathrm{~Hz}, 3 \mathrm{H}), 0.86(\mathrm{t}, J=7.1 \mathrm{~Hz}, 3 \mathrm{H}) ;{ }^{13} \mathrm{C} \mathrm{NMR}\left(125 \mathrm{MHz}, \mathrm{CDCl}_{3}\right): \square 170.5,144.9$, 120.0, 93.0, 45.3, 33.2, 28.5, 25.6, 23.1, 23.0, 20.4, 19.6, 13.9; IR $\left(\mathrm{CH}_{2} \mathrm{Cl}_{2}\right.$ cast, $\left.\mathrm{cm}^{-1}\right): 2960$, 1767, 1659, 1111; HRMS (ES, $m / z$ ) Calcd for $\mathrm{C}_{13} \mathrm{H}_{22} \mathrm{O}_{2} \mathrm{Na}$ : 233.151750. Found: 233.151637; Anal. Calcd for $\mathrm{C}_{13} \mathrm{H}_{22} \mathrm{O}_{2}$ : C, 74.22; H, 10.56. Found: C, 73.60; H, 10.86 .

\subsubsection{7 (4R $\left.R^{\star}, 5 R^{\star}\right)$-4-Butyl-4-methyl-3-methylene-5-(2-methylpropyl)-dihydro-furan-2-one 2ah}

A solution of allylboronate 1e $(126 \mathrm{mg}, 0.405 \mathrm{mmol})$ and isovaleraldehyde $(54 \mathrm{mg}$, $0.62 \mathrm{mmol})$ in toluene $(0.8 \mathrm{~mL})$ was treated with $\mathrm{Sc}(\mathrm{OTf})_{3}(22 \mathrm{mg}, 0.045 \mathrm{mmol})$ and stirred at room temperature under Ar for $24 \mathrm{~h}$. Reaction was diluted with water $(5 \mathrm{~mL})$ and 
extracted with $\mathrm{Et}_{2} \mathrm{O}(3 \times 5 \mathrm{~mL})$. The combined extracts were washed with brine $(5 \mathrm{~mL})$, dried $\left(\mathrm{Na}_{2} \mathrm{SO}_{4}\right)$ and concentrated to give the crude product $(88 \mathrm{mg})$. Flash chromatography (5\% $\mathrm{Et}_{2} \mathrm{O} / \mathrm{Hexanes,} 10 \mathrm{~g} \mathrm{SiO}_{2}$ ) gave the pure product (48 $\mathrm{mg}, 0.21 \mathrm{mmol}, 53 \%$ ).

TLC (1\% EtOAc/Toluene, $\mathrm{KMnO}_{4}$ ): 0.20; ${ }^{1} \mathrm{H}$ NMR (500 MHz, $\mathrm{CDCl}_{3}$ ): $\square 6.18$ (s, $1 \mathrm{H}$ ), $5.38(\mathrm{~s}, 1 \mathrm{H}), 4.06(\mathrm{dd}, J=10.9 \mathrm{~Hz}, 2.2 \mathrm{~Hz}, 1 \mathrm{H}), 1.86(\mathrm{~m}, 1 \mathrm{H}), 1.59(\mathrm{~m}, 1 \mathrm{H}), 1.4-1.0(\mathrm{~m}$, $7 \mathrm{H}), 1.13(\mathrm{~s}, 3 \mathrm{H}), 0.97(\mathrm{~d}, J=6.7 \mathrm{~Hz}, 3 \mathrm{H}), 0.92(\mathrm{~d}, J=6.6 \mathrm{~Hz}, 3 \mathrm{H}), 0.86(\mathrm{t}, J=7.1 \mathrm{~Hz}$, $3 \mathrm{H}) ;{ }^{13} \mathrm{C}$ NMR (100 MHz, $\mathrm{CDCl}_{3}$ ): $\square 170.8,144.3,120.0,86.0,45.2,37.3,33.4,25.6,25.2$, 23.5, 23.1, 21.6, 21.1, 13.9; IR $\left(\mathrm{CH}_{2} \mathrm{Cl}_{2}\right.$ cast, $\left.\mathrm{cm}^{-1}\right): 2957,1769,1661,1111$; HRMS (EI, $m / z)$ Calcd for $\mathrm{C}_{14} \mathrm{H}_{24} \mathrm{O}_{2}$ : 224.17763. Found: 224.17738.

\subsubsection{8 $\left(4 R^{\star}, 5 R^{\star}\right)$-4,5-Dibutyl-4-methyl-3-methylene-dihydro-furan-2-one 2ai}

A solution of allylboronate 1e (126 mg, $0.406 \mathrm{mmol})$ and valeraldehyde $(53 \mathrm{mg}$, $0.61 \mathrm{mmol})$ in toluene $(0.8 \mathrm{~mL})$ was treated with $\mathrm{Sc}(\mathrm{OTf})_{3}(17 \mathrm{mg}, 0.034 \mathrm{mmol})$ and stirred at room temperature under $\mathrm{Ar}$ for $24 \mathrm{~h}$. Reaction was diluted with water $(5 \mathrm{~mL})$ and extracted with $\mathrm{Et}_{2} \mathrm{O}(3 \times 5 \mathrm{~mL})$. The combined extracts were washed with brine $(5 \mathrm{~mL})$, dried $\left(\mathrm{Na}_{2} \mathrm{SO}_{4}\right)$ and concentrated to give the crude product $(160 \mathrm{mg})$. Flash chromatography (10\% EtOAc/Hexanes, $10 \mathrm{~g} \mathrm{SiO}_{2}$ ) gave the pure product (47 $\mathrm{mg}$, $0.21 \mathrm{mmol}, 51 \%$ ). A further $10 \mathrm{mg}$ of product was obtained which was contaminated with trace amounts of valeraldehyde pinacol acetal. Overall yield: $57 \mathrm{mg}, 0.25 \mathrm{mmol}, 62 \%$.

TLC (10\% EtOAc/Hexanes, $\mathrm{KMnO}_{4}$ ): 0.38; ${ }^{1} \mathrm{H}$ NMR (500 MHz, $\left.\mathrm{CDCl}_{3}\right): \square 6.18(\mathrm{~s}, 1 \mathrm{H})$, $5.38(\mathrm{~s}, 1 \mathrm{H}), 3.98(\mathrm{dd}, J=10.6 \mathrm{~Hz}, 2.9 \mathrm{~Hz}, 1 \mathrm{H}), 1.56(\mathrm{~m}, 3 \mathrm{H}), 1.35(\mathrm{~m}, 5 \mathrm{H}), 1.24(\mathrm{~m}, 2 \mathrm{H})$, $1.16(\mathrm{~m}, 2 \mathrm{H}), 1.14(\mathrm{~s}, 3 \mathrm{H}), 0.90(\mathrm{t}, J=7.0 \mathrm{~Hz}, 3 \mathrm{H}), 0.85(\mathrm{t}, J=7.0 \mathrm{~Hz}, 3 \mathrm{H}) ;{ }^{13} \mathrm{C}$ NMR (125 MHz, $\mathrm{CDCl}_{3}$ ): $\square 170.7,144.5,120.0,88.1,45.2,33.6,28.8,28.4,25.6,23.1,22.5,21.5$, 13.9, 13.8; IR $\left(\mathrm{CH}_{2} \mathrm{Cl}_{2}\right.$ cast, $\left.\mathrm{cm}^{-1}\right): 3093,2957,1770,1660,1106$; HRMS (EI, $\left.m / z\right)$ Calcd for $\mathrm{C}_{14} \mathrm{H}_{24} \mathrm{O}_{2}:$ 224.17763. Found: 224.17647; Anal. Calcd for $\mathrm{C}_{14} \mathrm{H}_{24} \mathrm{O}_{2}: \mathrm{C}, 74.94$; H, 10.80. Found: C, 74.45; H, 10.87 .

\subsubsection{9 $\left(4 R^{*}, 5 S^{*}\right)-5$-Benzyloxymethyl-4-methyl-3-methylene-dihydro-furan-2-one 2aj}

A solution of allylboronate 1a $(67 \mathrm{mg}, 0.29 \mathrm{mmol})$ and benzyloxyacetaldehyde $(47 \mathrm{mg}, 0.32 \mathrm{mmol})$ in toluene $(0.6 \mathrm{~mL})$ was treated with $\mathrm{Sc}(\mathrm{OTf})_{3}(15 \mathrm{mg}, 0.031 \mathrm{mmol})$ and stirred at room temperature under Ar for $25 \mathrm{~h}$. Reaction was diluted with water $(5 \mathrm{~mL})$ and extracted with $\mathrm{Et}_{2} \mathrm{O}(3 \times 5 \mathrm{~mL})$. The combined extracts were washed with brine $(5 \mathrm{~mL})$, dried $\left(\mathrm{Na}_{2} \mathrm{SO}_{4}\right)$ and concentrated to give the crude product $(73 \mathrm{mg})$. Flash chromatography $\left(10 \% \mathrm{Et}_{2} \mathrm{O} / \mathrm{Hexanes}, 5 \mathrm{~g} \mathrm{SiO}_{2}\right.$ ) gave the pure product (22 $\left.\mathrm{mg}, 0.095 \mathrm{mmol}, 33 \%\right)$. 
TLC (25\% Et $\left.\mathrm{E}_{2} \mathrm{O} / \mathrm{Hexanes,} \mathrm{KMnO}_{4}\right): 0.10 ;{ }^{1} \mathrm{H} \mathrm{NMR}\left(500 \mathrm{MHz}, \mathrm{CDCl}_{3}\right): \square 7.34(\mathrm{~m}, 2 \mathrm{H})$, $7.25(\mathrm{~m}, 3 \mathrm{H}), 6.16(\mathrm{~d}, J=3.2 \mathrm{~Hz}, 1 \mathrm{H}), 5.45(\mathrm{~d}, J=2.8 \mathrm{~Hz}, 1 \mathrm{H}), 4.64(\mathrm{~m}, 1 \mathrm{H}), 4.51$ (AB, $J=12.0 \mathrm{~Hz}, 1 \mathrm{H}), 4.49(\mathrm{AB}, J=12.3 \mathrm{~Hz}, 1 \mathrm{H}), 3.63(\mathrm{dq}, J=10.8 \mathrm{~Hz}, 4.2 \mathrm{~Hz}, 2 \mathrm{H}), 3.59$ (m, $1 \mathrm{H}), 1.24(\mathrm{~d}, J=7.0 \mathrm{~Hz}, 3 \mathrm{H}) ;{ }^{13} \mathrm{C} \mathrm{NMR}\left(125 \mathrm{MHz}, \mathrm{CDCl}_{3}\right): \square$ 170.2, 140.4, 137.6, 128.4, 127.7, 127.4, 119.2, 79.1, 73.6, 68.7, 36.4, 13.1; IR $\left(\mathrm{CH}_{2} \mathrm{Cl}_{2}\right.$ cast, $\left.\mathrm{cm}^{-1}\right)$ : 3030, 1764, 1663, 1121, 731, 698; HRMS (EI, $m / z$ ) Calcd for $\mathrm{C}_{14} \mathrm{H}_{16} \mathrm{O}_{3}$ : 232.10994. Found: 232.1010; Anal. Calcd for $\mathrm{C}_{14} \mathrm{H}_{16} \mathrm{O}_{3}$ : C, 72.38; H, 6.96. Found: C, 72.01; H, 6.87.

\subsubsection{Lewis acid catalysed reactions with phenylboronic acid scavenging}

\subsubsection{1 $\left(4 R^{\star}, 5 S^{\star}\right)$-4-Ethyl-4-methyl-3-methylene-5-phenethyl-dihydro-furan-2-one 2ab}

A solution of allylboronate 1c $(100 \mathrm{mg}, 0.354 \mathrm{mmol})$ and hydrocinnamaldehyde (46 mg, $0.34 \mathrm{mmol})$ in toluene $(0.7 \mathrm{~mL})$ was treated with $\mathrm{Sc}(\mathrm{OTf})_{3}(20 \mathrm{mg}, 0.041 \mathrm{mmol})$ and phenylboronic acid (44 mg, $0.36 \mathrm{mmol}$ ). The resulting mixture was stirred at room temperature under Ar for $18 \mathrm{~h}$. Reaction was diluted with water $(5 \mathrm{~mL})$ and extracted with $\mathrm{Et}_{2} \mathrm{O}(3 \times 5 \mathrm{~mL})$. The combined extracts were washed with brine $(5 \mathrm{~mL})$, dried $\left(\mathrm{Na}_{2} \mathrm{SO}_{4}\right)$ and concentrated to give the crude product $(138 \mathrm{mg})$. Flash chromatography $\left(25 \% \mathrm{Et}_{2} \mathrm{O} / \mathrm{Hexanes}, 16 \mathrm{~g} \mathrm{SiO}_{2}\right.$ ) gave the pure product (69 $\left.\mathrm{mg}, 0.28 \mathrm{mmol}, 82 \%\right)$. The spectral characteristics for this compound were described in Section 6.5.1.1.

\subsubsection{2 $\left(4 R^{\star}, 5 R^{\star}\right)$-5-Benzyloxymethyl-4-ethyl-4-methyl-3-methylene-dihydro-furan-2-one 2ac}

A solution of allylboronate $1 \mathrm{c}(86 \mathrm{mg}, 0.30 \mathrm{mmol})$ and benzyloxyacetaldehyde (45 mg, $0.30 \mathrm{mmol})$ in toluene $(0.6 \mathrm{~mL})$ was treated with $\mathrm{Sc}(\mathrm{OTf})_{3}(18 \mathrm{mg}, 0.037 \mathrm{mmol})$ and phenylboronic acid (40 $\mathrm{mg}, 0.32 \mathrm{mmol})$. The resulting mixture was stirred at room temperature under Ar for $19 \mathrm{~h}$. Reaction was diluted with water $(5 \mathrm{~mL})$ and extracted with $\mathrm{Et}_{2} \mathrm{O}(3 \times 5 \mathrm{~mL})$. The combined extracts were washed with brine $(5 \mathrm{~mL})$, dried $\left(\mathrm{Na}_{2} \mathrm{SO}_{4}\right)$ and concentrated to give the crude product $(134 \mathrm{mg})$. Flash chromatography $\left(25 \% \mathrm{Et}_{2} \mathrm{O} / \mathrm{Hexanes}, 6 \mathrm{~g} \mathrm{SiO}_{2}\right.$ ) gave the pure product (42 $\mathrm{mg}, 0.16 \mathrm{mmol}, 54 \%$ ). The spectral characteristics for this compound were described in Section 6.5.1.2.

\subsubsection{3 $\left(4 R^{*}, 5 S^{*}\right)$-4-Ethyl-4-methyl-3-methylene-5-(2-methylpropyl)-dihydro-furan-2-one 2ad}

A solution of allylboronate $1 c(104 \mathrm{mg}, 0.37 \mathrm{mmol})$ and isovaleraldehyde (32 $\mathrm{mg}$, $0.38 \mathrm{mmol})$ in toluene $(0.7 \mathrm{~mL})$ was treated with $\mathrm{Sc}(\mathrm{OTf})_{3}(18 \mathrm{mg}, 0.037 \mathrm{mmol})$ and 
phenylboronic acid $(42 \mathrm{mg}, 0.35 \mathrm{mmol})$. The resulting slurry was stirred at room temperature under Ar for $12 \mathrm{~h}$. Reaction was diluted with water $(5 \mathrm{~mL})$ and extracted with $\mathrm{Et}_{2} \mathrm{O}(3 \times 5 \mathrm{~mL})$. The combined extracts were washed with brine $(5 \mathrm{~mL})$, dried $\left(\mathrm{Na}_{2} \mathrm{SO}_{4}\right)$ and concentrated to give the crude product $(122 \mathrm{mg})$. Flash chromatography (50\% $\mathrm{CH}_{2} \mathrm{Cl}_{2} / \mathrm{Hexanes,} 6 \mathrm{~g} \mathrm{SiO}_{2}$ ) gave the pure product (48 $\mathrm{mg}, 0.24 \mathrm{mmol}, 66 \%$ ). The spectral characteristics for this compound were described in Section 6.5.1.3.

\subsubsection{4 $\left(4 R^{\star}, 5 S^{\star}\right)$-5-Benzyloxymethyl-4-methyl-3-methylene-dihydro-furan-2-one 2aj}

A solution of allylboronate 1a $(70 \mathrm{mg}, 0.30 \mathrm{mmol})$ and benzyloxyacetaldehyde $(50 \mathrm{mg}, 0.33 \mathrm{mmol})$ in toluene $(0.6 \mathrm{~mL})$ was treated with $\mathrm{Sc}(\mathrm{OTf})_{3}(15 \mathrm{mg}, 0.037 \mathrm{mmol})$ and phenylboronic acid $(46 \mathrm{mg}, 0.38 \mathrm{mmol})$. The resulting mixture was stirred at room temperature under Ar for $25 \mathrm{~h}$. Reaction was diluted with water $(5 \mathrm{~mL})$ and extracted with $\mathrm{Et}_{2} \mathrm{O}(3 \times 5 \mathrm{~mL})$. The combined extracts were washed with brine $(5 \mathrm{~mL})$, dried $\left(\mathrm{Na}_{2} \mathrm{SO}_{4}\right)$ and concentrated to give the crude product $(96 \mathrm{mg})$. Flash chromatography (25\% $\mathrm{Et}_{2} \mathrm{O} / \mathrm{Hexanes}, 6 \mathrm{~g} \mathrm{SiO}_{2}$ ) gave the pure product ( $42 \mathrm{mg}, 0.18 \mathrm{mmol}, 60 \%$ ). The spectral characteristics for this compound were described in Section 6.5.1.9.

\subsubsection{Enantioselective catalysed reactions}

\subsubsection{Enantioselective allylboration with 8-phenylmenthol allylboronate $11 \mathrm{e}$ under $\mathrm{Sc}(\mathrm{OTf})_{3}$ catalysis}

Chiral allylboronate 11e (55 mg, $0.12 \mathrm{mmol})$ and decanal (29 $\mathrm{mg}, 0.18 \mathrm{mmol})$ in toluene $(0.25 \mathrm{~mL})$ was treated with $\mathrm{Sc}(\mathrm{OTf})_{3}(5 \mathrm{mg}, 0.01 \mathrm{mmol})$ and stirred at room temperature under Ar. After $24 \mathrm{~h}$, the mixture was diluted with $\mathrm{CH}_{2} \mathrm{Cl}_{2}(5 \mathrm{~mL})$, treated with a spatula tip of pTSA, and stirred for $3 \mathrm{~h}$. The reaction was quenched with $\mathrm{NaHCO}_{3(\text { aq })}$ $(5 \mathrm{~mL})$ and extracted with $\mathrm{Et}_{2} \mathrm{O}(3 \times 5 \mathrm{~mL})$. The combined ether layers were washed with brine $(5 \mathrm{~mL})$, dried $\left(\mathrm{Na}_{2} \mathrm{SO}_{4}\right)$ and concentrated to give the crude product $(34 \mathrm{mg})$, which was purified by flash chromatography ( $1 \%$ EtOAc/Toluene, $8 \mathrm{~g}$ silica) to give the enantioenriched product $\mathbf{2 d}(9 \mathrm{mg}, 29 \%)$. Chiral HPLC analysis (CHIRALPAK AD-RH, $50 \%$ isopropanol/water, $0.3 \mathrm{~mL} / \mathrm{min}$ ) showed that the enantiomeric excess of the product was $10 \%$ for the $(R)$-enantiomer. 


\subsubsection{Enantioselective allylboration with 8-phenylmenthol allylboronate $11 \mathrm{e}$ under $\mathrm{Cu}(\mathrm{OTf})_{2}$ catalysis}

This reaction was performed following the procedure outlined in Section 6.5.3.1, using chiral allylboronate 11e (59 mg, $0.13 \mathrm{mmol}$ ), decanal (30 mg, $0.18 \mathrm{mmol})$, and $\mathrm{Cu}(\mathrm{OTf})_{2}(5 \mathrm{mg}, 0.01 \mathrm{mmol})$. Work-up and purification gave lactone $2 \mathrm{~d}$ in $93 \%$ yield and with a $71 \%$ ee for the $(S)$-enantiomer.

\subsubsection{Enantioselective allylboration with 8-phenylmenthol allylboronate 11e under $\mathrm{Yb}(\mathrm{OTf})_{3}$ catalysis}

This reaction was performed following the procedure outlined in Section 6.5.3.1, using chiral allylboronate 11e (63 $\mathrm{mg}, 0.14 \mathrm{mmol})$, decanal $(32 \mathrm{mg}, 0.20 \mathrm{mmol})$, and $\mathrm{Yb}(\mathrm{OTf})_{3}(9 \mathrm{mg}, 0.01 \mathrm{mmol})$. Work-up and purification gave lactone 2d in $30 \%$ yield and with a $31 \%$ ee for the $(S)$-enantiomer.

\subsubsection{Enantioselective allylboration with chiral allylboronate 18 under $\mathrm{Sc}(\mathrm{OTf})_{3}$ catalysis}

Chiral allylboronate $18(120 \mathrm{mg}, 0.30 \mathrm{mmol})$ and decanal $(70 \mathrm{mg}, 0.45 \mathrm{mmol})$ in toluene $(0.6 \mathrm{~mL})$ was treated with $\mathrm{Sc}(\mathrm{OTf})_{3}(19 \mathrm{mg}, 0.04 \mathrm{mmol})$ and stirred at room temperature under Ar. After $24 \mathrm{~h}$, the mixture was diluted with water $(5 \mathrm{~mL})$ and extracted with $\mathrm{Et}_{2} \mathrm{O}(3 \times 5 \mathrm{~mL})$. The combined ether layers were washed with brine $(5 \mathrm{~mL})$, dried $\left(\mathrm{Na}_{2} \mathrm{SO}_{4}\right)$ and concentrated to give the crude product $(145 \mathrm{mg})$, which was purified by flash chromatography ( $25 \% \mathrm{Et}_{2} \mathrm{O} / \mathrm{Hexanes,} 7 \mathrm{~g}$ silica) to give the enantioenriched product $\mathbf{2 d}$ (40 mg, 53\%). Chiral HPLC analysis (CHIRALPAK AD-RH, 50\% isopropanol/water, $0.3 \mathrm{~mL} / \mathrm{min}$ ) showed that the enantiomeric excess of the product was $3 \%$ for the $(R)$-enantiomer.

\subsubsection{Enantioselective allylboration with chiral allylboronate 18 under $\mathrm{Cu}(\mathrm{OTf})_{2}$ catalysis}

This reaction was performed following the procedure outlined in Section 6.5.3.4, using chiral allylboronate 18 (119 $\mathrm{mg}, 0.30 \mathrm{mmol})$, decanal $(73 \mathrm{mg}, 0.46 \mathrm{mmol})$, and $\mathrm{Cu}(\mathrm{OTf})_{2}(12 \mathrm{mg}, 0.33 \mathrm{mmol})$. Work-up and purification gave lactone $\mathbf{2 d}$ in $31 \%$ yield and with a $13 \%$ ee for the $(R)$-enantiomer. 


\subsubsection{Enantioselective allylboration with chiral allylboronate 18 under $\mathrm{Yb}(\mathrm{OTf})_{3}$ catalysis}

This reaction was performed following the procedure outlined in Section 6.5.3.4, using chiral allylboronate 18 (132 mg, $0.334 \mathrm{mmol})$, decanal $(78 \mathrm{mg}, 0.50 \mathrm{mmol})$, and $\mathrm{Yb}(\mathrm{OTf})_{3}(19 \mathrm{mg}, 0.30 \mathrm{mmol})$. Work-up and purification gave lactone 2d in $37 \%$ yield and with a $21 \%$ ee for $(R)$-enantiomer.

\section{Further functionalization of lactones 2}

\section{1 -Methylbutyrolactones 27 via hydrogenation of the exocyclic double bond}

\subsection{1 $\left(3 R^{*}, 4 R^{*}, 5 S^{*}\right)-4-E$ thyl-3,4-dimethyl-5-nonyl-dihydro-furan-2-one 27 a}

A solution of lactone $2 \mathbf{e}(107 \mathrm{mg}, 0.401 \mathrm{mmol})$ in $\mathrm{EtOH}(10 \mathrm{~mL})$ was hydrogenated at $1 \mathrm{~atm}$ with $10 \% \mathrm{Pd} / \mathrm{C}(18 \mathrm{mg})$. After $60 \mathrm{~h}$ the mixture was filtered through Celite ${ }^{\circledR}$ and the residue was washed with ethanol $(6 \times 10 \mathrm{~mL})$. The combined filtrates were concentrated and the resulting oil was purified by Kugelrohr distillation $\left(200{ }^{\circ} \mathrm{C}, 0.1\right.$ torr $)$ to give the product as a white solid (92 $\mathrm{mg}, 0.34 \mathrm{mmol}, 85 \%)$.

${ }^{1} \mathrm{H}$ NMR (500 MHz, $\mathrm{CDCl}_{3}$ ): 3.99 (dd, $\left.J=10.1 \mathrm{~Hz}, 2.6 \mathrm{~Hz}, 1 \mathrm{H}\right), 2.46(\mathrm{q}, J=7.2 \mathrm{~Hz}$, $1 \mathrm{H}), 1.58(\mathrm{~m}, 1 \mathrm{H}), 1.46(\mathrm{~m}, 3 \mathrm{H}), 1.25(\mathrm{~m}, 12 \mathrm{H}), 1.2-0.8(\mathrm{~m}, 5 \mathrm{H}), 1.07(\mathrm{~d}, J=7.2 \mathrm{~Hz}, 3 \mathrm{H})$, $0.91(\mathrm{t}, J=7.6 \mathrm{~Hz}, 3 \mathrm{H}), 0.78(\mathrm{~s}, 3 \mathrm{H}) ;{ }^{13} \mathrm{C} \mathrm{NMR}\left(125 \mathrm{MHz}, \mathrm{CDCl}_{3}\right): \mathrm{1}$ 178.8, 86.0, 45.3, 44.8, 31.9, 30.0, 29.6, 29.5, 29.4, 29.3, 27.1, 22.7, 14.4, 14.2, 9.1, 9.0; IR $\left(\mathrm{CH}_{2} \mathrm{Cl}_{2}\right.$ cast, $\mathrm{cm}^{-1}$ ): 2925, 1776, 1204; HRMS (EI, $\mathrm{m} / z$ ) Calcd for $\mathrm{C}_{17} \mathrm{H}_{32} \mathrm{O}_{2}:$ 268.24023. Found: 268.23970; Anal. Calcd for $\mathrm{C}_{17} \mathrm{H}_{32} \mathrm{O}_{2}$ : C, 76.04; H, 12.04. Found: C, 76.12; H, 12.13.

\subsection{2 $\left(3 R^{\star}, 4 R^{\star}, 5 R^{\star}\right)$-4-Ethyl-3,4-dimethyl-5-phenyl-dihydro-furan-2-one $27 \mathrm{~b}$}

This compound as prepared according to the procedure in Section 7.1.1, using lactone $2 \mathbf{f}(106 \mathrm{mg}, 0.491 \mathrm{mmol})$ and $10 \% \mathrm{Pd} / \mathrm{C}(11 \mathrm{mg})$ in $\mathrm{EtOH}(10 \mathrm{~mL})$, except the reaction was left for $5 \mathrm{~d}$. Kugelrohr distillation $\left(250{ }^{\circ} \mathrm{C}, 0.1\right.$ torr) afforded the pure product (92 mg, $0.42 \mathrm{mmol}, 86 \%$ ).

${ }^{1} \mathrm{H}$ NMR (400 MHz, $\left.\mathrm{CDCl}_{3}\right): 7.34(\mathrm{~m}, 3 \mathrm{H}), 7.25(\mathrm{~m}, 2 \mathrm{H}), 5.16(\mathrm{~s}, 1 \mathrm{H}), 2.71$ (q, $J=7.2 \mathrm{~Hz}, 1 \mathrm{H}), 1.56(\mathrm{~m}, 2 \mathrm{H}), 1.15(\mathrm{~d}, J=7.2 \mathrm{~Hz}, 3 \mathrm{H}), 1.01(\mathrm{t}, J=7.6 \mathrm{~Hz}, 3 \mathrm{H}), 0.54(\mathrm{~s}$, $3 \mathrm{H}) ;{ }^{13} \mathrm{C}$ NMR (125 MHz, $\mathrm{CDCl}_{3}$ ): $\square$ 178.6, 135.0, 128.08, 128.06, 126.2, 85.6, 47.5, 43.8, 29.0, 16.0, 9.5, 8.8; IR $\left(\mathrm{CH}_{2} \mathrm{Cl}_{2}\right.$ cast, $\left.\mathrm{cm}^{-1}\right): 3032,2971,1782,1658,1134,742,701$; 
HRMS (EI, $m / z)$ Calcd for $\mathrm{C}_{14} \mathrm{H}_{18} \mathrm{O}_{2}:$ 218.13068. Found: 218.12994; Anal. Calcd for $\mathrm{C}_{14} \mathrm{H}_{18} \mathrm{O}_{2}: \mathrm{C}, 77.01 ; \mathrm{H}, 8.33$. Found: $\mathrm{C}, 75.71 ; \mathrm{H}, 8.19$.

\subsection{3 $\left(3 R^{*}, 4 S^{*}, 5 R^{*}\right)-4-(2-M e t h y \mid p r o p y l)-5-(4-m e t h o x y p h e n y l)-3,4-d i m e t h y l-d i h y d r o-f u r a n-2-$ one $27 \mathrm{c}$}

This compound as prepared according to the procedure in Section 7.1.1, using lactone $2 \mathbf{k}$ (48 $\mathrm{mg}, 0.18 \mathrm{mmol})$ and $10 \% \mathrm{Pd} / \mathrm{C}(12 \mathrm{mg})$ in EtOH $(5 \mathrm{~mL})$, except the reaction was left for $48 \mathrm{~h}$. Kugelrohr distillation $\left(250{ }^{\circ} \mathrm{C}, 0.1\right.$ torr) afforded the pure solid product (39 mg, $0.14 \mathrm{mmol}, 81 \%$ ).

TLC (25\% Et $\mathrm{E}_{2} \mathrm{O} / \mathrm{Hexanes,} \mathrm{PMA):} \mathrm{0.21;}{ }^{1} \mathrm{H}$ NMR (500 $\left.\mathrm{MHz}, \mathrm{CDCl}_{3}\right): \square 7.21(\mathrm{AB}$, $J=8.6 \mathrm{~Hz}, 2 \mathrm{H}), 6.88(\mathrm{AB}, J=8.7 \mathrm{~Hz}, 2 \mathrm{H}), 4.97(\mathrm{~s}, 1 \mathrm{H}), 3.80(\mathrm{~s}, 3 \mathrm{H}), 2.50$ (q, $J=7.2 \mathrm{~Hz}$, $1 \mathrm{H}), 1.26(\mathrm{~s}, 3 \mathrm{H}), 1.16(\mathrm{~d}, J=7.2 \mathrm{~Hz}, 3 \mathrm{H}), 1.1-0.9(\mathrm{~m}, 3 \mathrm{H}), 0.71(\mathrm{~d}, J=6.4 \mathrm{~Hz}, 3 \mathrm{H}), 0.54$ $(\mathrm{d}, J=6.4 \mathrm{~Hz}, 3 \mathrm{H}) ;{ }^{13} \mathrm{C} \mathrm{NMR}\left(125 \mathrm{MHz}, \mathrm{CDCl}_{3}\right)$ : $\square$ 178.4, 159.4, 127.3, 127.2, 113.6, 89.1, 55.3, 49.2, 48.4, 37.4, 25.3, 25.1, 23.6, 21.9, 9.4; IR $\left(\mathrm{CH}_{2} \mathrm{Cl}_{2}\right.$ cast, $\left.\mathrm{cm}^{-1}\right): 2958,1778$, 1613, 1385, 1367, 1252, 829; HRMS (EI, $\mathrm{m} / z$ ) Calcd for $\mathrm{C}_{17} \mathrm{H}_{24} \mathrm{O}_{3}$ : 276.17255. Found: 276.17279; Anal. Calcd for $\mathrm{C}_{17} \mathrm{H}_{24} \mathrm{O}_{3}$ : C, 73.86; H, 8.77. Found: C, 73.46; H, 8.93.

\subsection{4 $\left(3 R^{*}, 4 R^{*}, 5 S^{\star}\right)$-4-Ethyl-3,4-dimethyl-5-phenethyl-dihydro-furan-2-one $27 \mathrm{~d}$}

This compound as prepared by Melissa Chee according to the procedure outlined in Section 7.1.1, using lactone $2 \mathbf{a b}(448 \mathrm{mg}, 1.82 \mathrm{mmol})$ and $10 \% \mathrm{Pd} / \mathrm{C}(50 \mathrm{mg})$ in $\mathrm{EtOH}$ $(18 \mathrm{~mL})$, except the reaction was left for $6 \mathrm{~h}$. Kugelrohr distillation $\left(250{ }^{\circ} \mathrm{C}, 0.1\right.$ torr $)$ afforded the pure product (393 $\mathrm{mg}, 1.60 \mathrm{mmol}, 88 \%$ ).

TLC (10\% EtOAc/Hexanes, $\mathrm{KMnO}_{4}$ ): 0.25; ${ }^{1} \mathrm{H}$ NMR (400 MHz, $\left.\mathrm{CDCl}_{3}\right)$ : $7.2(\mathrm{~m}, 5 \mathrm{H})$, $4.0(\mathrm{dd}, J=11.1 \mathrm{~Hz}, 2.1 \mathrm{~Hz}, 1 \mathrm{H}), 2.9(\mathrm{~m}, 1 \mathrm{H}), 2.7(\mathrm{~m}, 1 \mathrm{H}), 2.45(\mathrm{q}, J=14.4 \mathrm{~Hz}, 1 \mathrm{H})$, $1.8(\mathrm{~m}, 2 \mathrm{H}), 1.4(\mathrm{~m}, 2 \mathrm{H}), 1.05(\mathrm{~d}, J=15 \mathrm{~Hz}, 3 \mathrm{H}), 0.95$ (t, $J=7.0 \mathrm{~Hz}, 3 \mathrm{H}), 0.8(\mathrm{~s}, 3 \mathrm{H})$; ${ }^{13} \mathrm{C}$ NMR $\left(125 \mathrm{MHz}, \mathrm{CDCl}_{3}\right)$ : $\square$ 178.7, 141.1, 128.5, 128.4, 126.1, 84.6, 45.1, 44.7, 32.9, 31.3, 29.8, 14.3, 9.0, 8.9; IR $\left(\mathrm{CH}_{2} \mathrm{Cl}_{2}\right.$ cast, $\left.\mathrm{cm}^{-1}\right)$ : 3084, 2979, 1755; HRMS (EI, $\left.m / z\right)$ Calcd for $\mathrm{C}_{16} \mathrm{H}_{22} \mathrm{O}_{2}$ : 246.16198. Found: 246.16177; Anal. Calcd for $\mathrm{C}_{16} \mathrm{H}_{22} \mathrm{O}_{2}:$ C, 78.00; H, 9.02. Found: C, 77.76; H, 9.22. 


\subsection{Reduction of butyrolactone 27 a to acyclic diol 28}

\subsection{1 $\left(2 R^{*}, 3 R^{*}, 4 S^{*}\right)-3$-Ethyl-2,3-dimethyltridecane-1,4-diol 28}

A solution of butyrolactone $27 \mathrm{a}(23 \mathrm{mg}, 0.084 \mathrm{mmol})$ in THF $(5 \mathrm{~mL})$ was treated with Red-Al (65\% solution in toluene, $0.55 \mathrm{~mL}, 0.18 \mathrm{mmol}$ ) and refluxed at $80^{\circ} \mathrm{C}$. After $14 \mathrm{~h}$ the mixture was brought to room temperature and carefully quenched with $\sim 3$ drops of water from a Pasteur pipette. The resulting mixture was passed through a plug of cotton wool. The residue was rinsed with EtOAc $(6 \times 2 \mathrm{~mL})$, and the combined filtrates were dried $\left(\mathrm{Na}_{2} \mathrm{SO}_{4}\right)$ and concentrated to give the crude product $(25 \mathrm{mg})$. The compound was further purified by Kugelrohr distillation $\left(250^{\circ} \mathrm{C}, 0.1\right.$ torr) to give the product as a colourless oil (18 mg, $0.068 \mathrm{mg}, 80 \%$ ).

TLC (20\% EtOAc/toluene, PMA): $0.29 ;{ }^{1} \mathrm{H}$ NMR (500 MHz, $\left.\mathrm{CDCl}_{3}\right): 03.63$ (m, 2H), $3.48(\mathrm{~m}, 1 \mathrm{H}), 2.49(\mathrm{br} \mathrm{s}, 2 \mathrm{H}), 1.76(\mathrm{~m}, 1 \mathrm{H}), 1.6-1.2(\mathrm{~m}, 18 \mathrm{H}+$ hydrocarbon impurities), $1.02(\mathrm{~d}, J=7.2 \mathrm{~Hz}, 3 \mathrm{H}), 0.88(\mathrm{~m}, 3 \mathrm{H}), 0.82(\mathrm{~s}, 3 \mathrm{H}), 0.81(\mathrm{t}, J=5.2 \mathrm{~Hz}, 3 \mathrm{H}) ;{ }^{13} \mathrm{C} \mathrm{NMR}$ (125 MHz, $\mathrm{CDCl}_{3}$ ): $\square 77.6,64.9,41.7,41.1,31.9,31.8,29.72,29.71,29.6,29.3,29.0,27.4$, 22.7, 17.9, 14.1, 13.8, 8.0; IR $\left(\mathrm{CH}_{2} \mathrm{Cl}_{2}\right.$ cast, $\left.\mathrm{cm}^{-1}\right)$ : 3345, 2925; HRMS (ES, $\left.\mathrm{m} / z\right)$ Calcd for $\mathrm{C}_{17} \mathrm{H}_{36} \mathrm{O}_{2} \mathrm{Na}$ : 295.26075. Found: 295.26057. 


\section{NMR spectra of 2-carboxyester-3,3-disubstituted allylboronates 1}
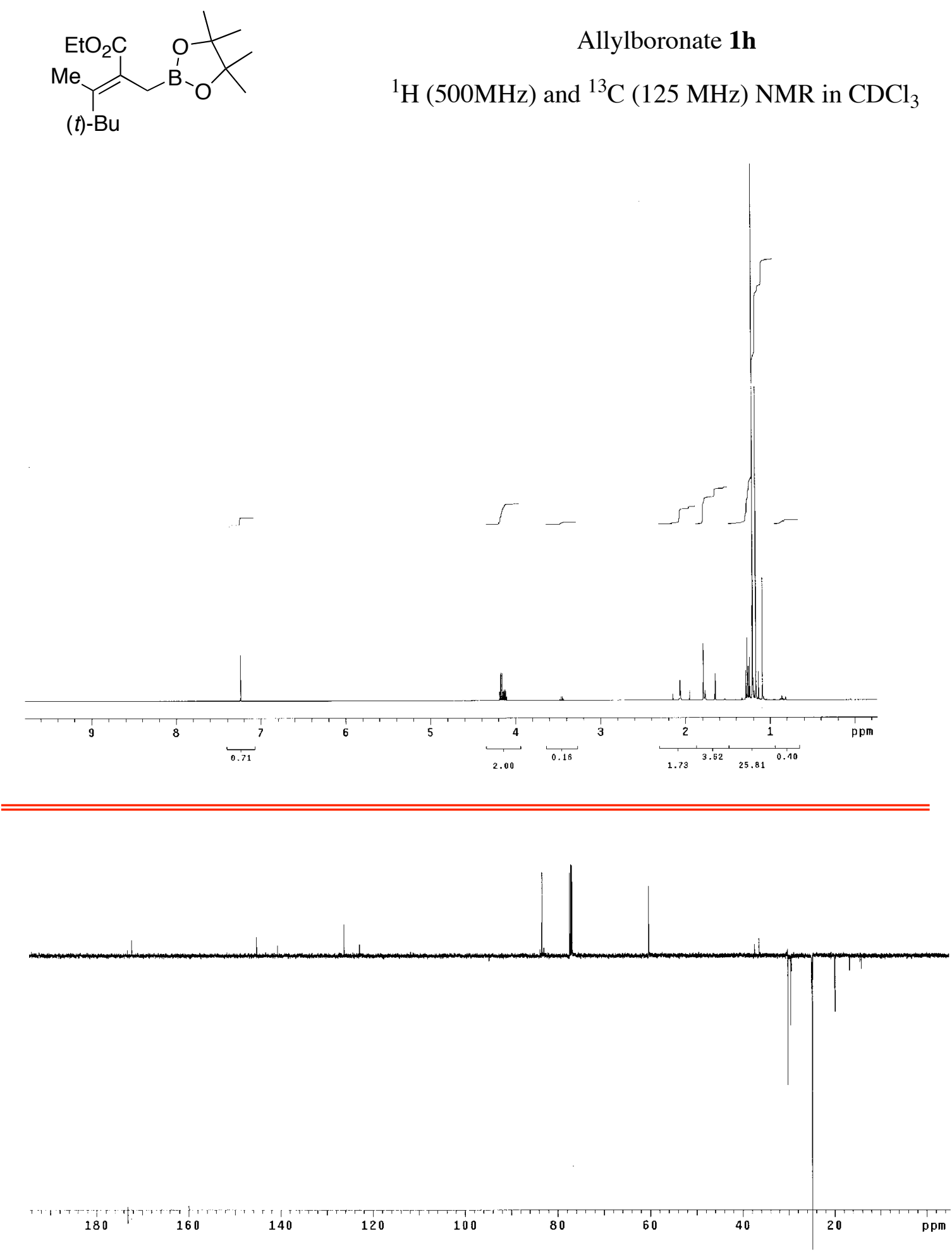


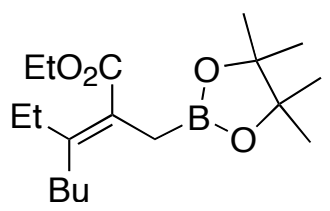

Allylboronate 1i

${ }^{1} \mathrm{H}(500 \mathrm{MHz})$ and ${ }^{13} \mathrm{C}(100 \mathrm{MHz}) \mathrm{NMR}$ in $\mathrm{CDCl}_{3}$
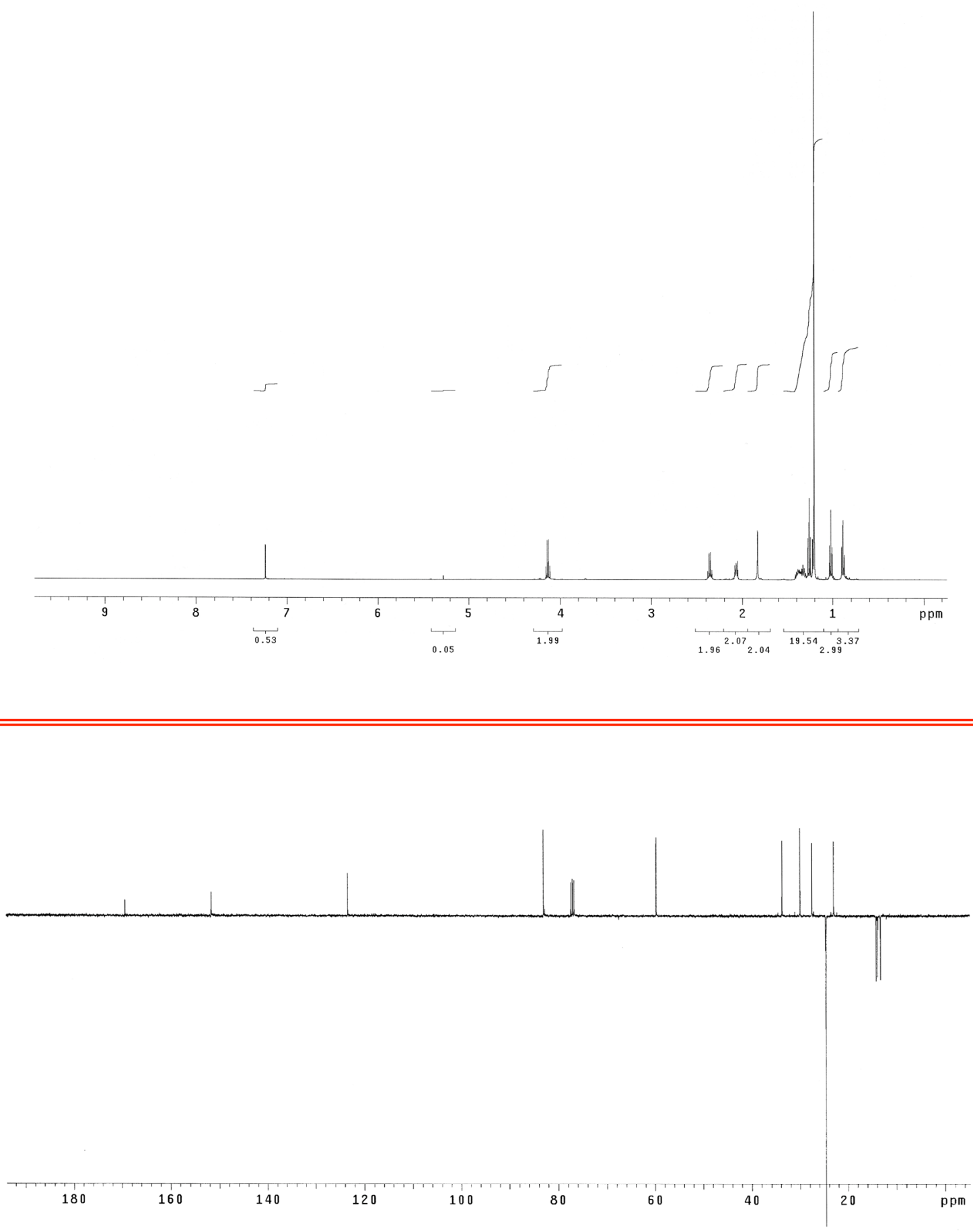


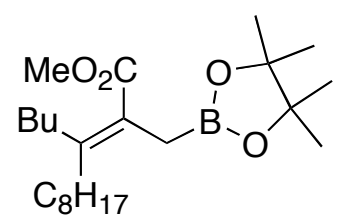

Allylboronate 1j

${ }^{1} \mathrm{H}(300 \mathrm{MHz})$ and ${ }^{13} \mathrm{C}(125 \mathrm{MHz}) \mathrm{NMR}$ in $\mathrm{CDCl}_{3}$
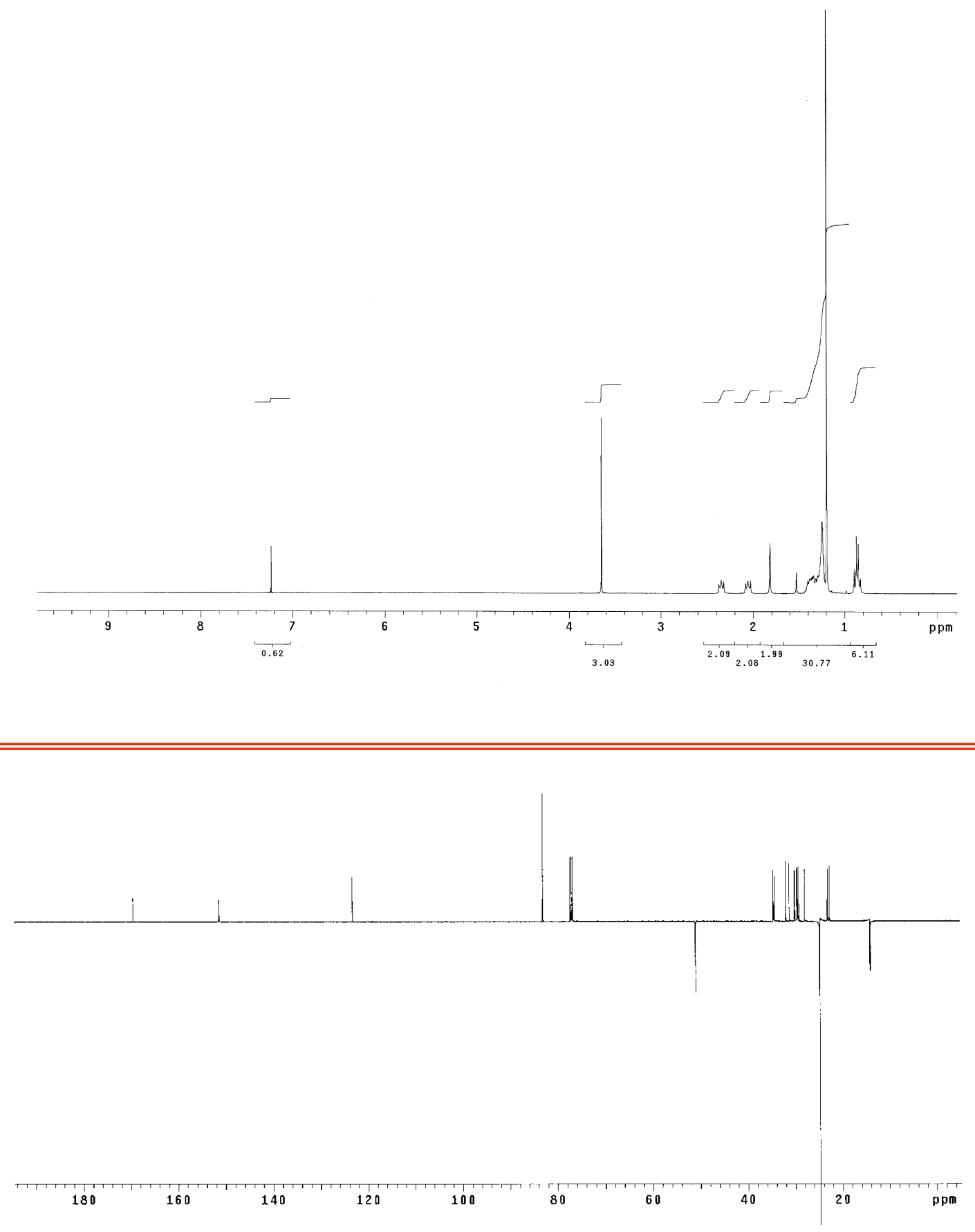

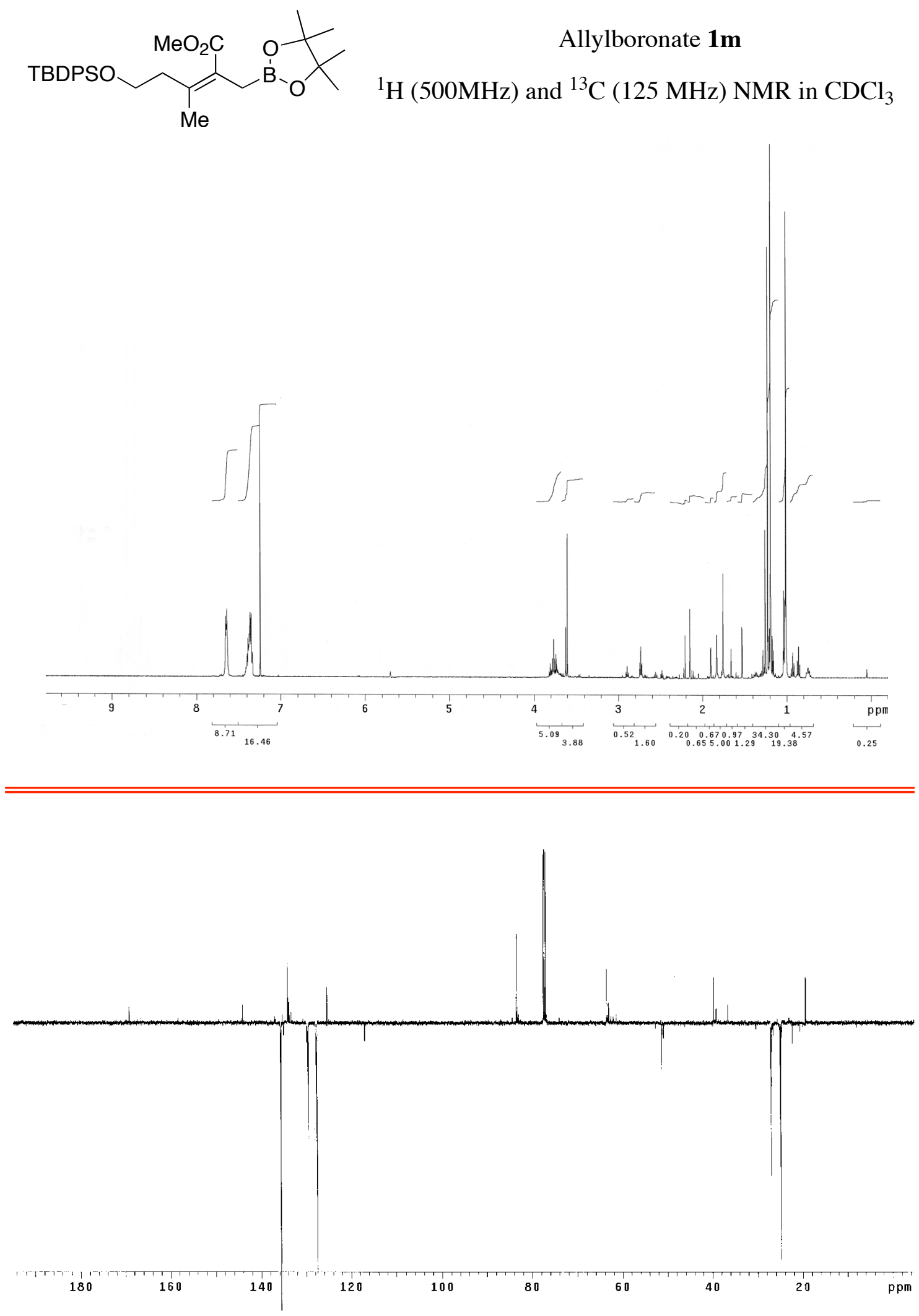

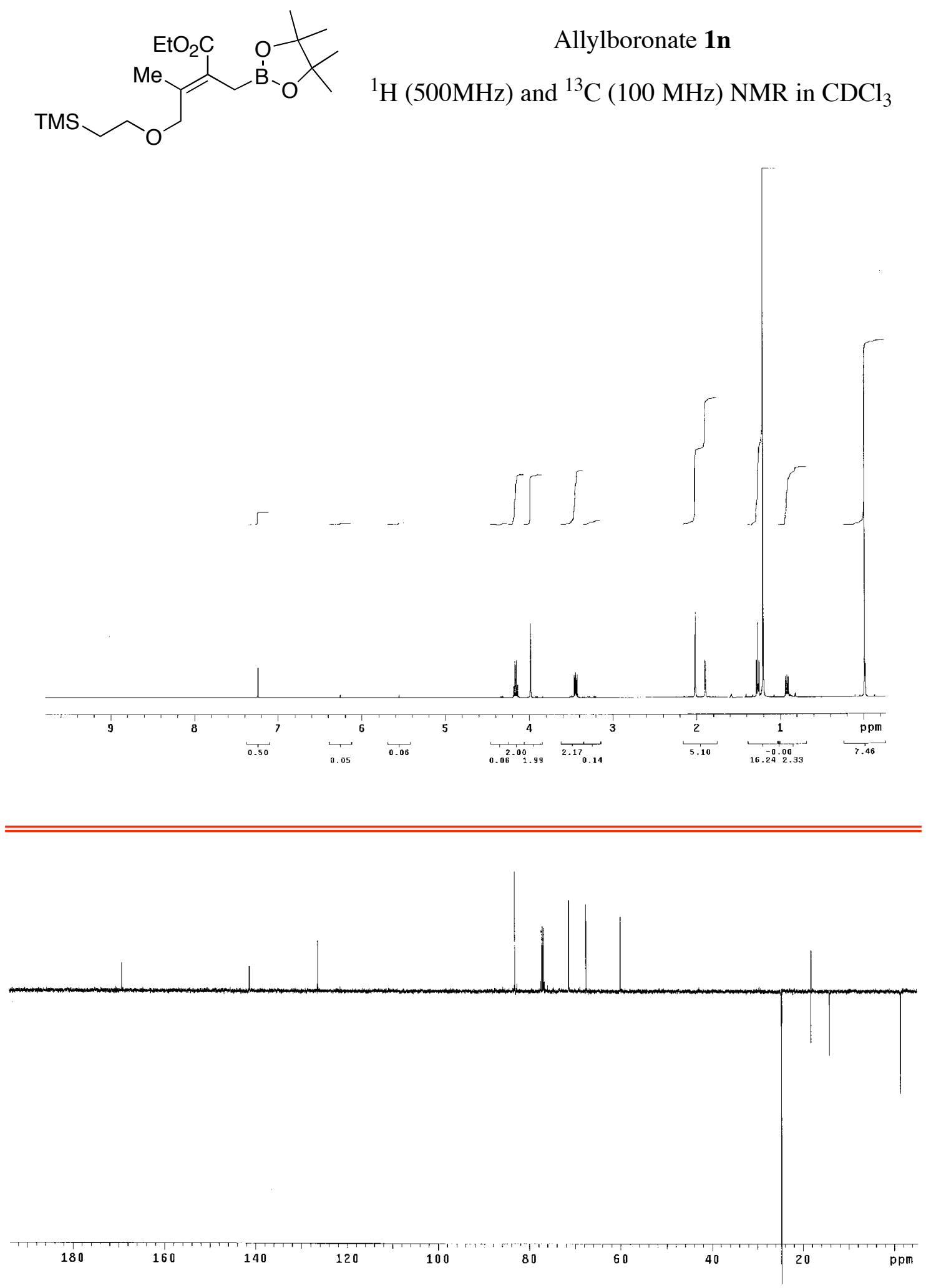

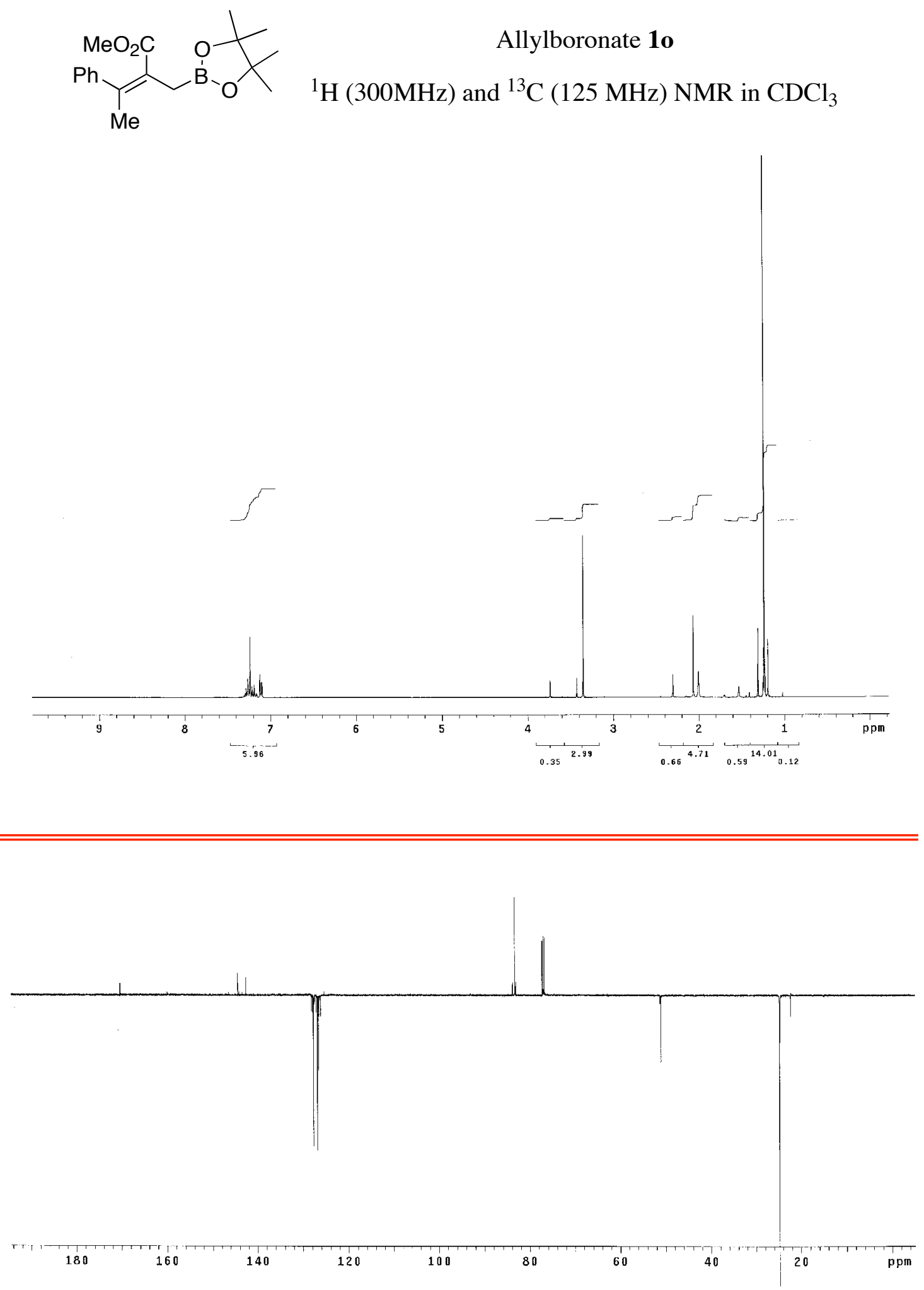
9 NMR spectra of exo-methylene butyrolactones 2

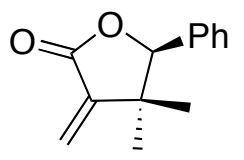

Lactone $\mathbf{2 b}$

${ }^{1} \mathrm{H}(300 \mathrm{MHz})$ and ${ }^{13} \mathrm{C}(75 \mathrm{MHz}) \mathrm{NMR}$ in $\mathrm{CDCl}_{3}$
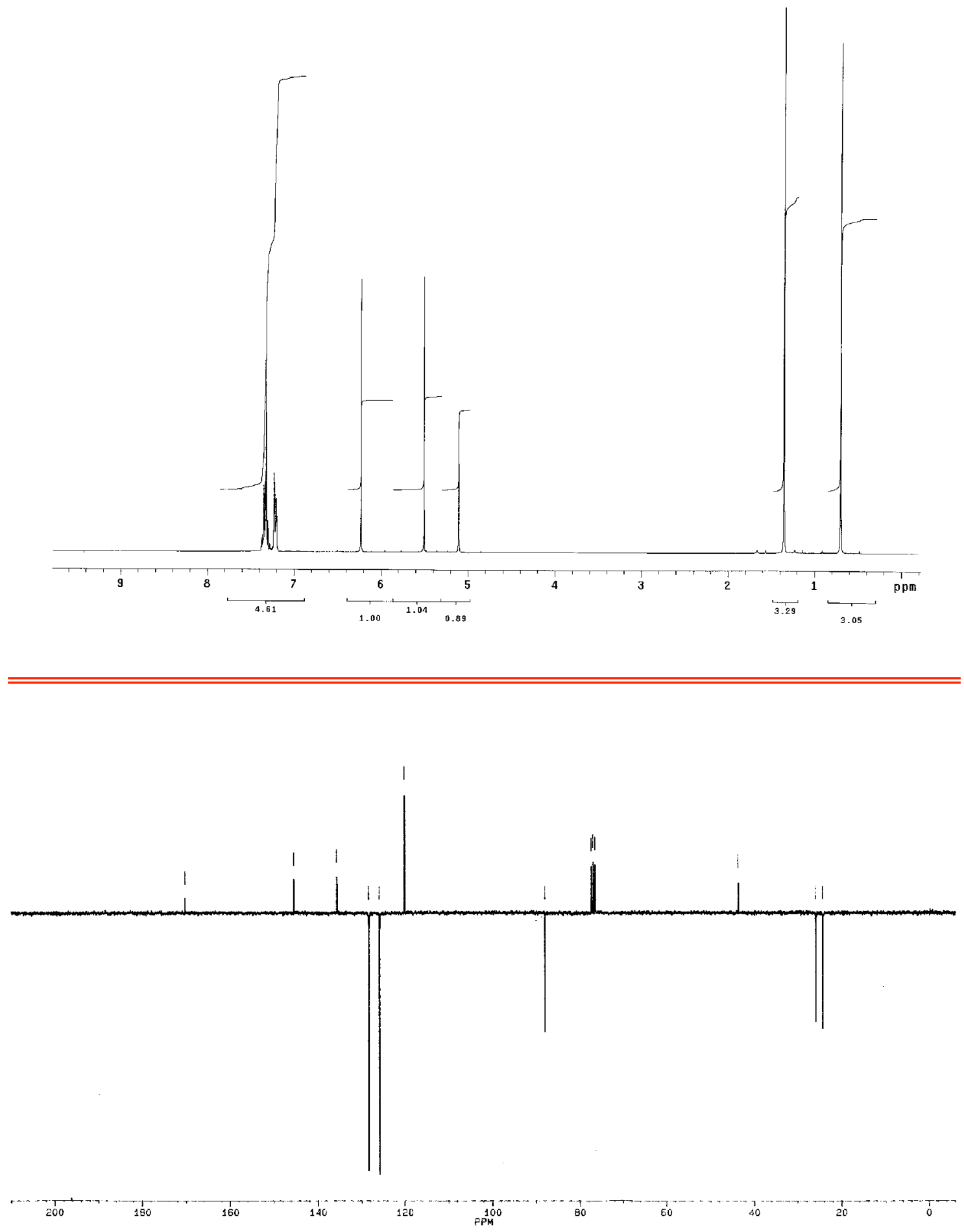


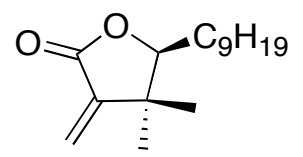

Lactone 2d

${ }^{1} \mathrm{H}(500 \mathrm{MHz})$ and ${ }^{13} \mathrm{C}(125 \mathrm{MHz}) \mathrm{NMR}$ in $\mathrm{CDCl}_{3}$
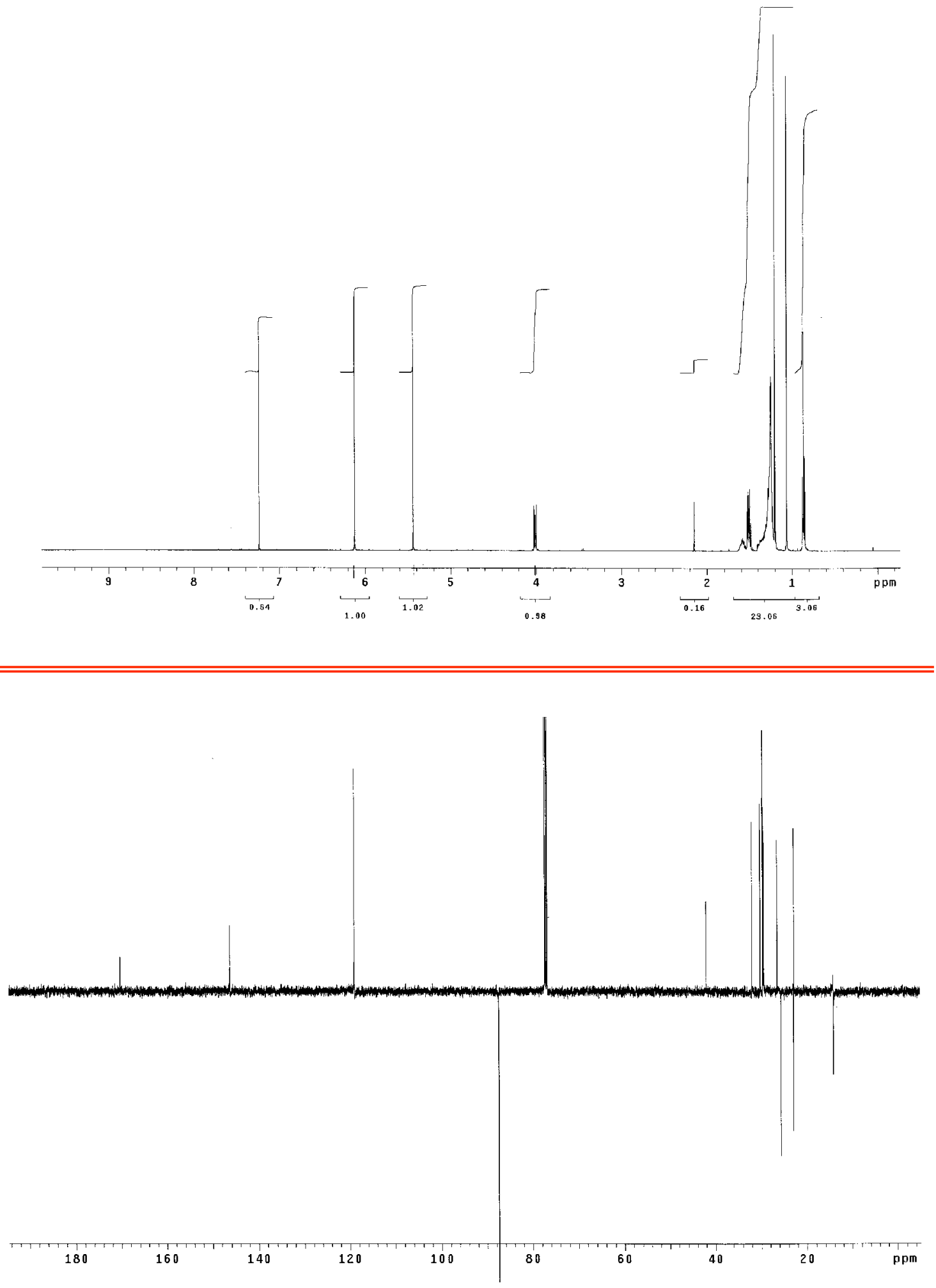

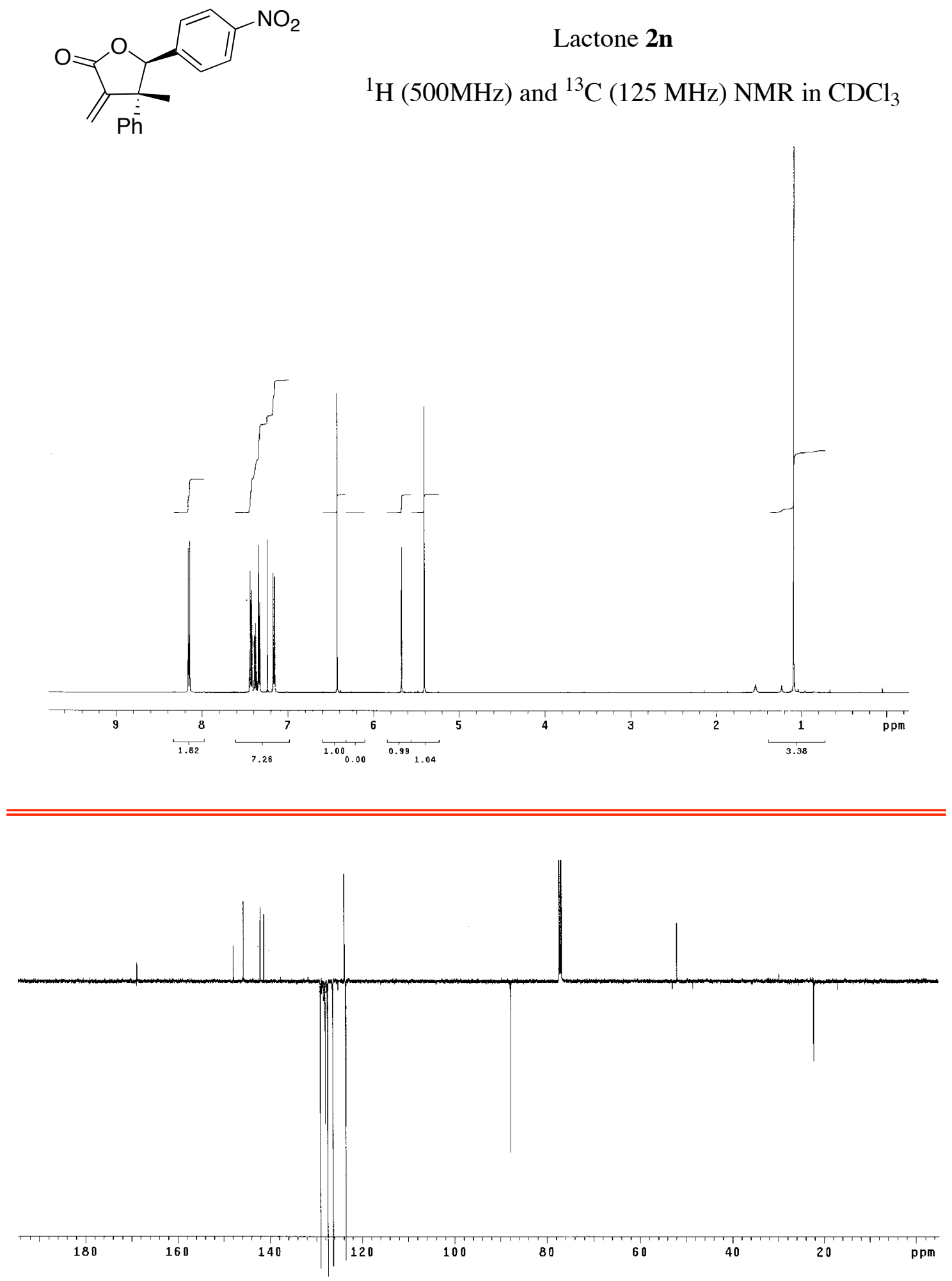


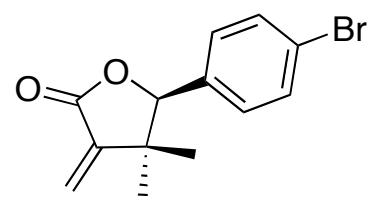

Lactone 20

${ }^{1} \mathrm{H}(500 \mathrm{MHz})$ and ${ }^{13} \mathrm{C}(125 \mathrm{MHz}) \mathrm{NMR}$ in $\mathrm{CDCl}_{3}$
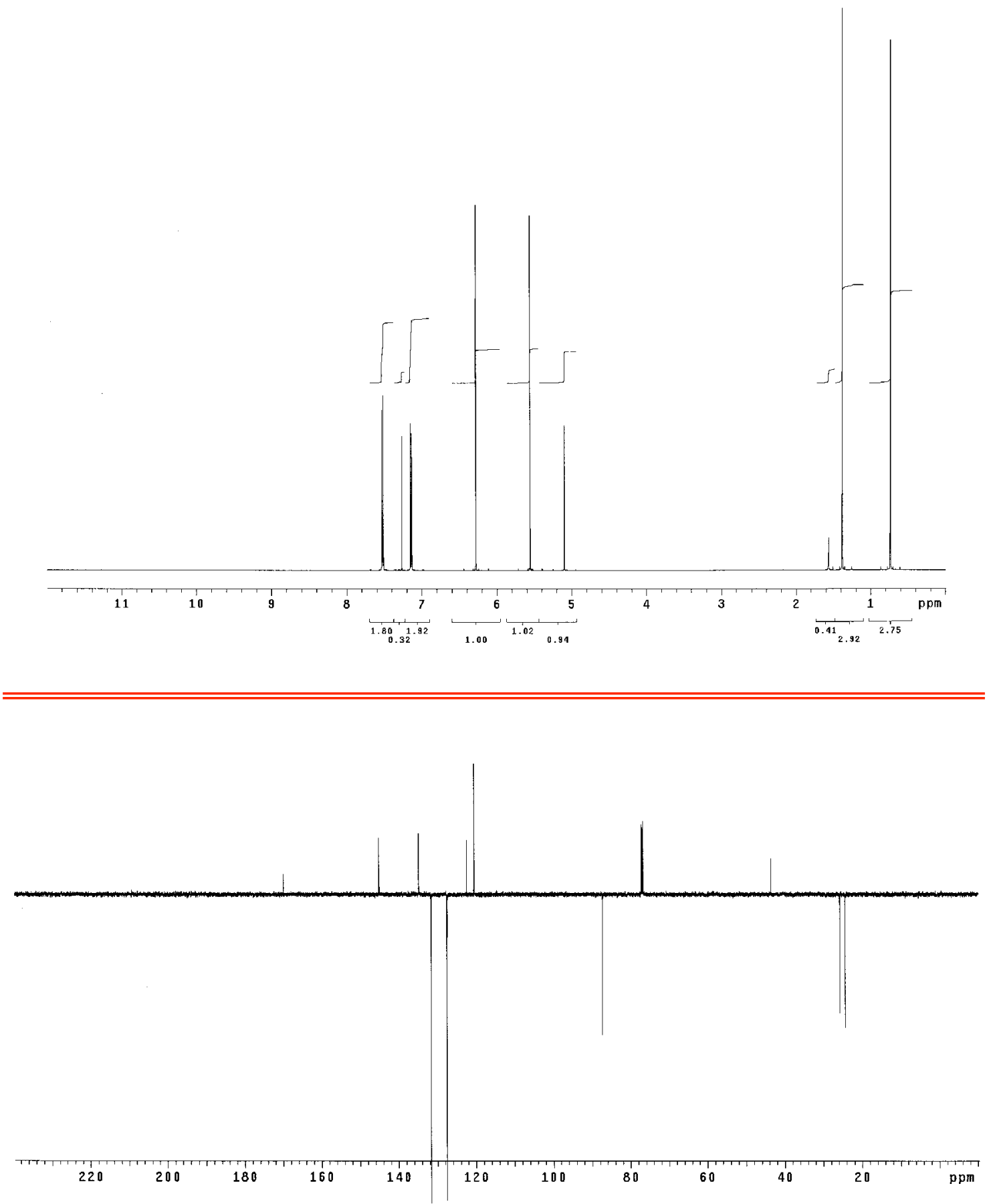
<smiles>C=C1C(=O)O[C@@H](c2ccc(OC(C)=O)cc2)[C@@]1(C)CC</smiles>

Lactone 2p

${ }^{1} \mathrm{H}(500 \mathrm{MHz})$ and ${ }^{13} \mathrm{C}(125 \mathrm{MHz})$ NMR in $\mathrm{CDCl}_{3}$
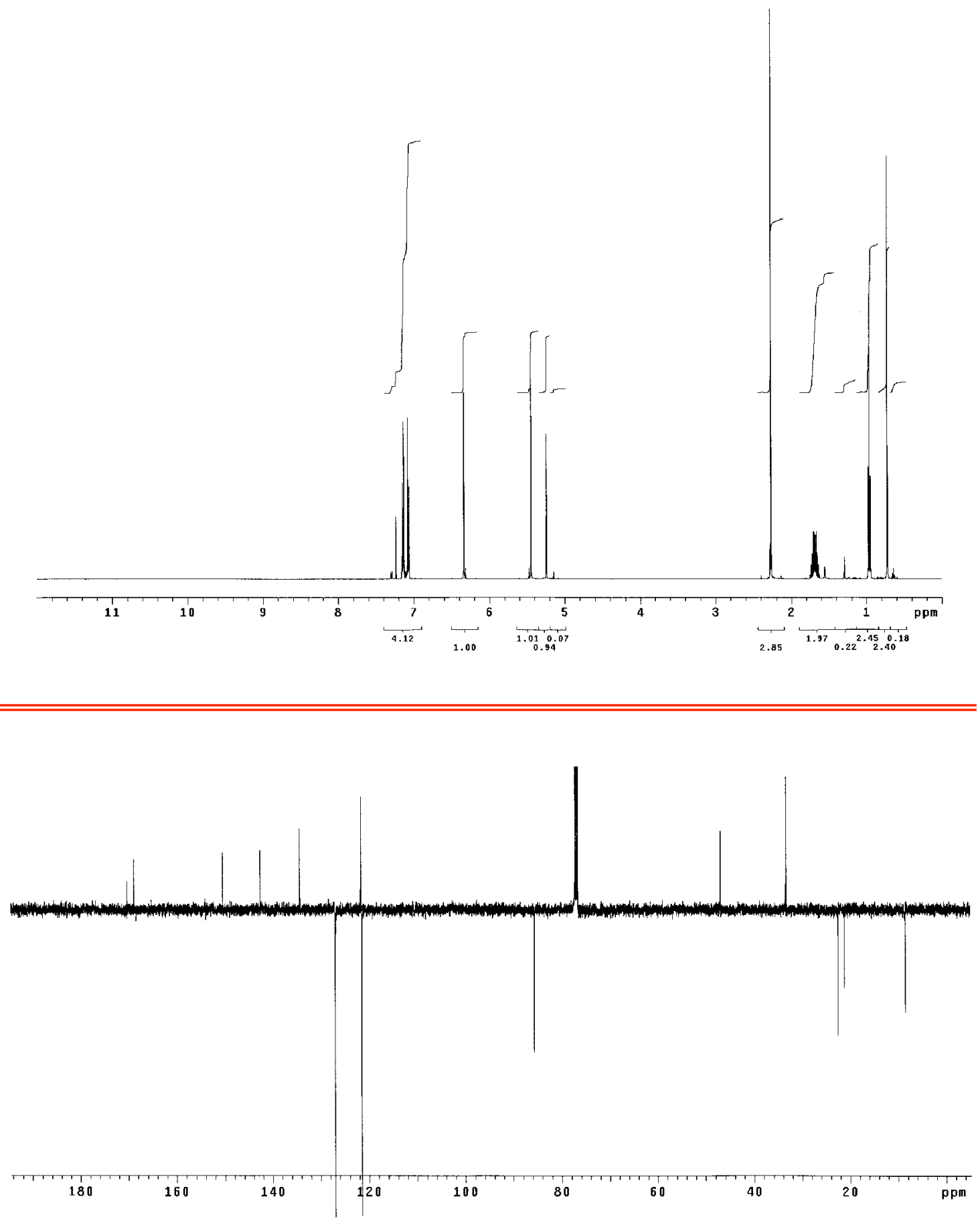
<smiles>C=C1C(=O)O[C@@H](c2cccc3ccccc23)C1(C)C</smiles>

Lactone $\mathbf{2 t}$

${ }^{1} \mathrm{H}(500 \mathrm{MHz})$ and ${ }^{13} \mathrm{C}(125 \mathrm{MHz}) \mathrm{NMR}$ in $\mathrm{CDCl}_{3}$
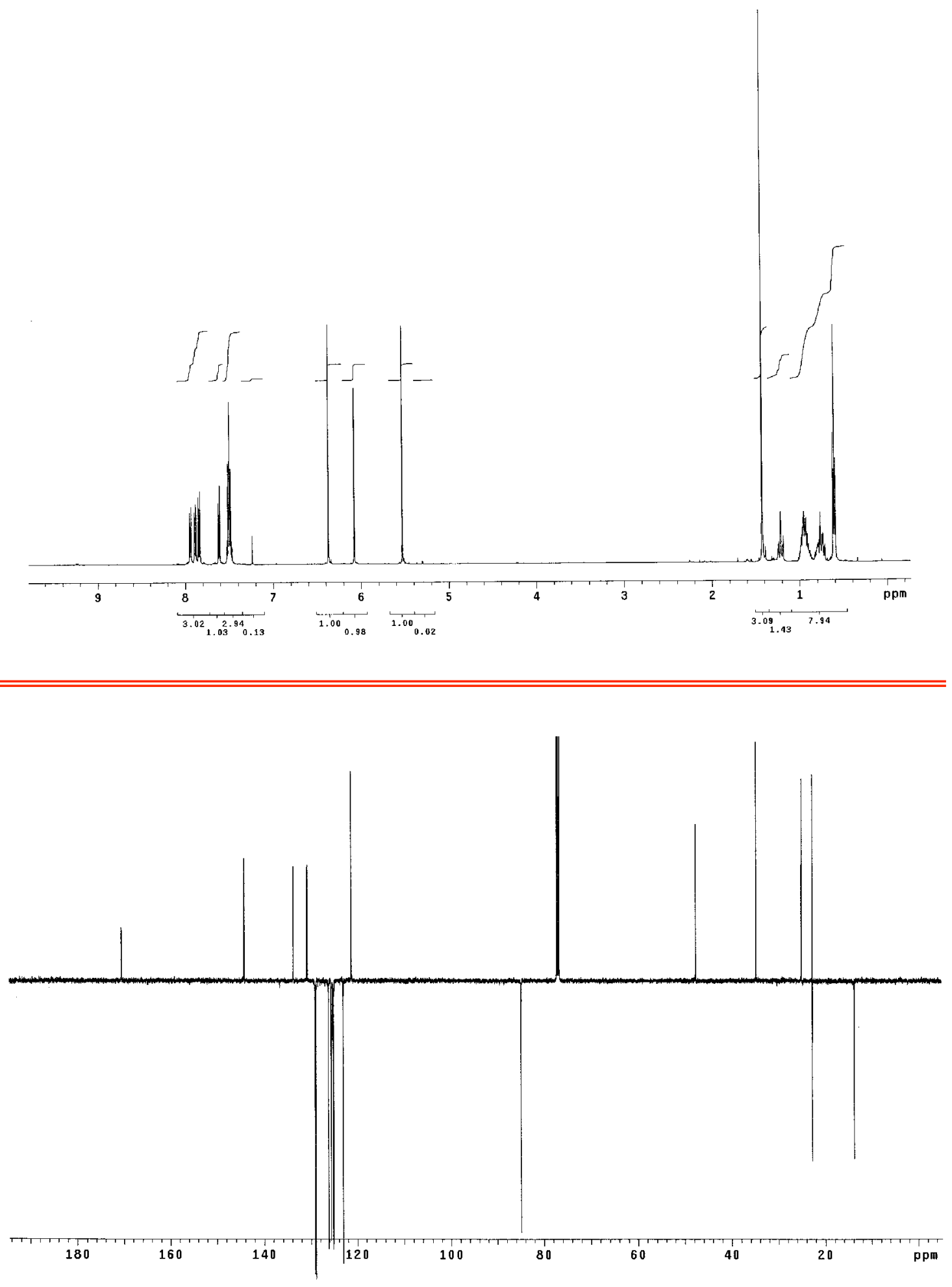


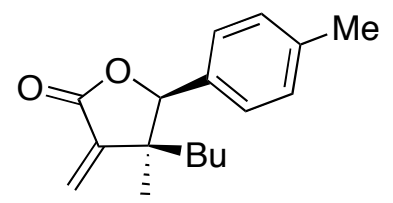

Lactone $\mathbf{2 u}$

${ }^{1} \mathrm{H}(400 \mathrm{MHz})$ and ${ }^{13} \mathrm{C}(100 \mathrm{MHz}) \mathrm{NMR}$ in $\mathrm{CDCl}_{3}$
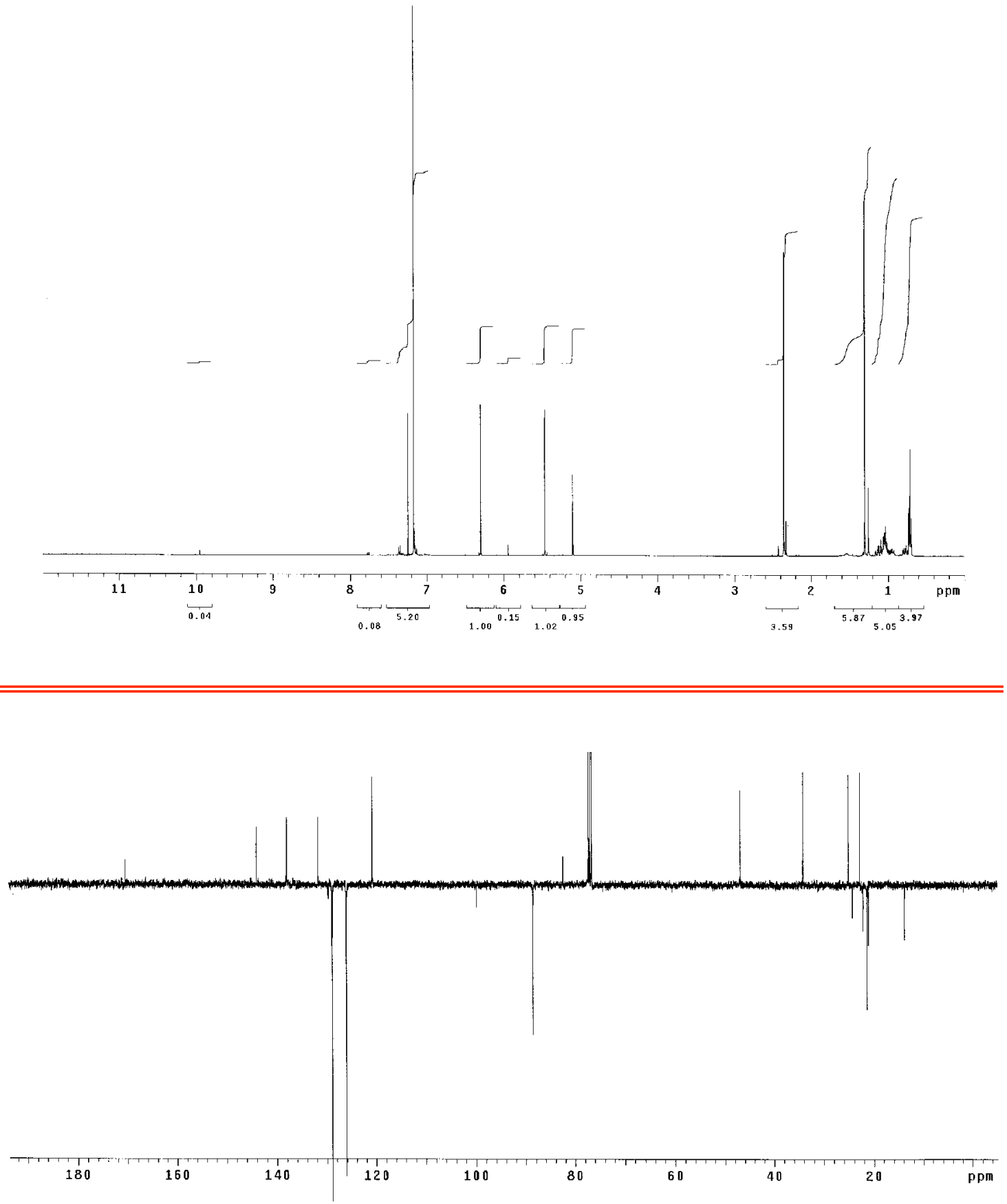


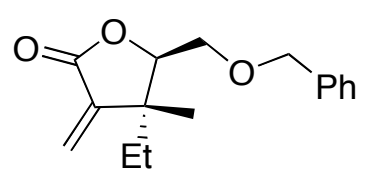

Lactone 2ac

${ }^{1} \mathrm{H}(500 \mathrm{MHz}) \mathrm{NMR}$ in $\mathrm{CDCl}_{3}$

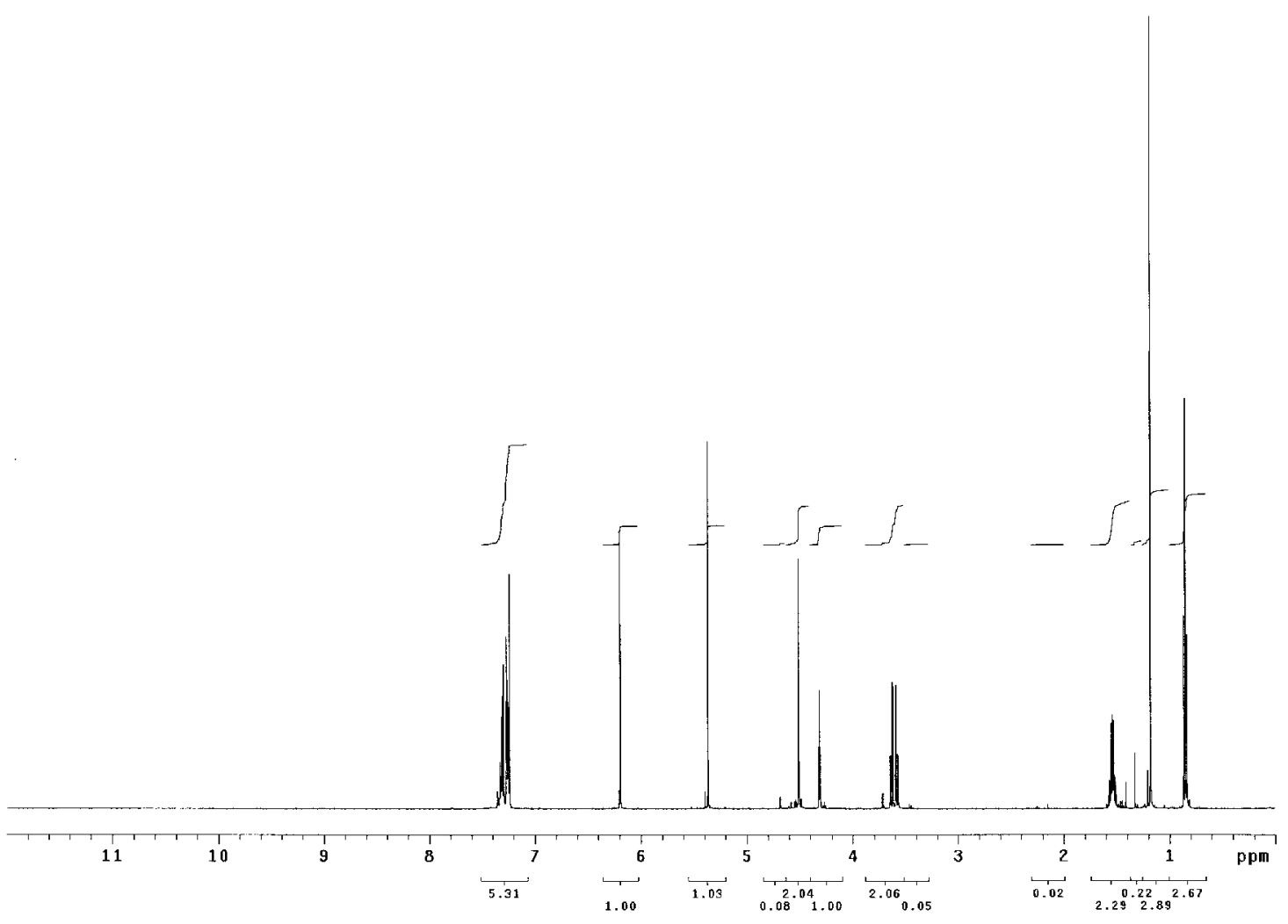




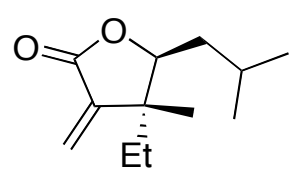

Lactone 2ad

${ }^{1} \mathrm{H}(500 \mathrm{MHz}) \mathrm{NMR}$ in $\mathrm{CDCl}_{3}$

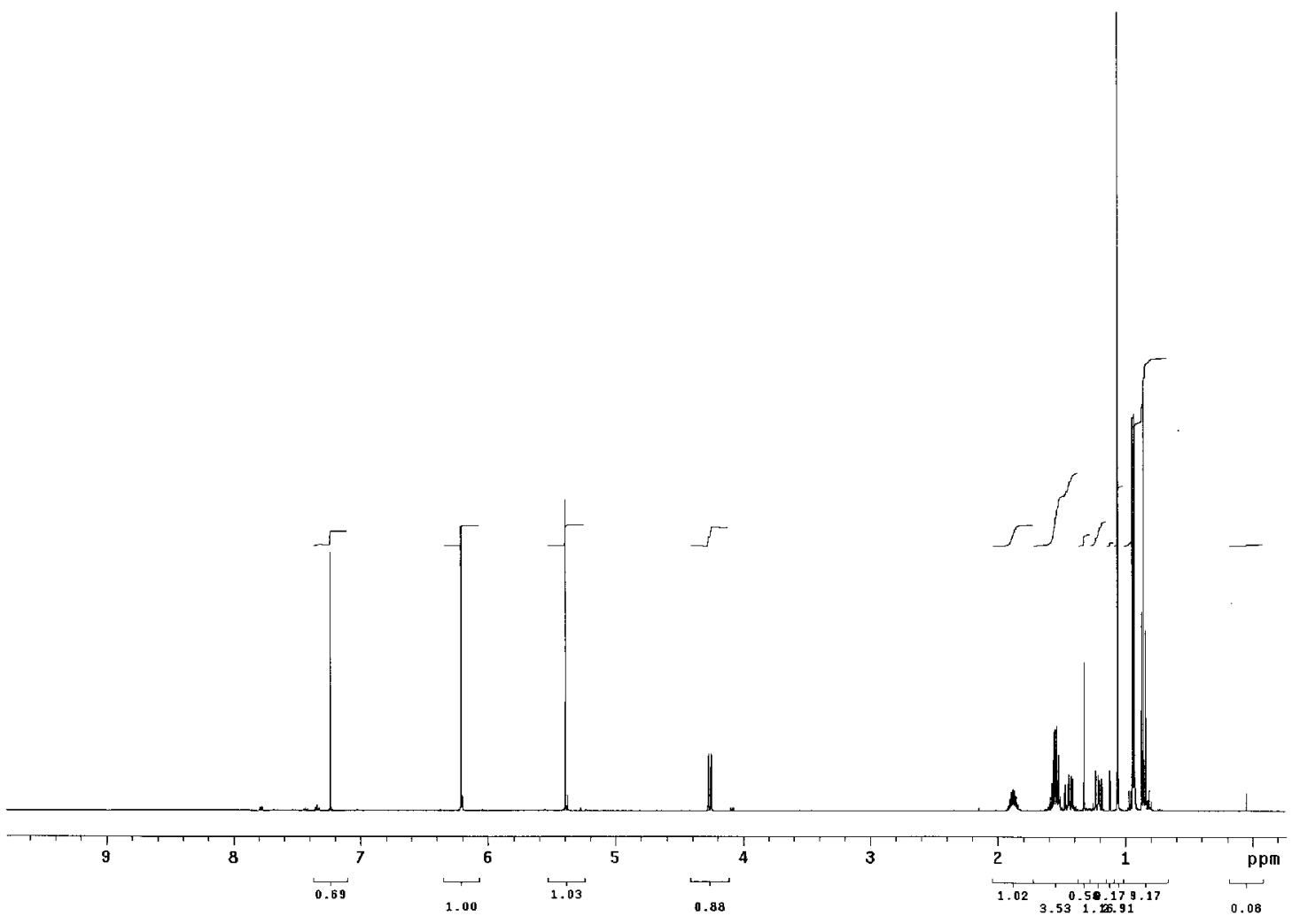




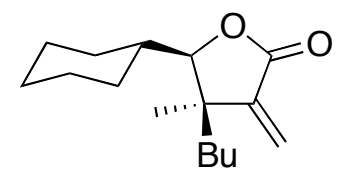

Lactone 2af

${ }^{1} \mathrm{H}(500 \mathrm{MHz})$ and ${ }^{13} \mathrm{C}(125 \mathrm{MHz}) \mathrm{NMR}$ in $\mathrm{CDCl}_{3}$
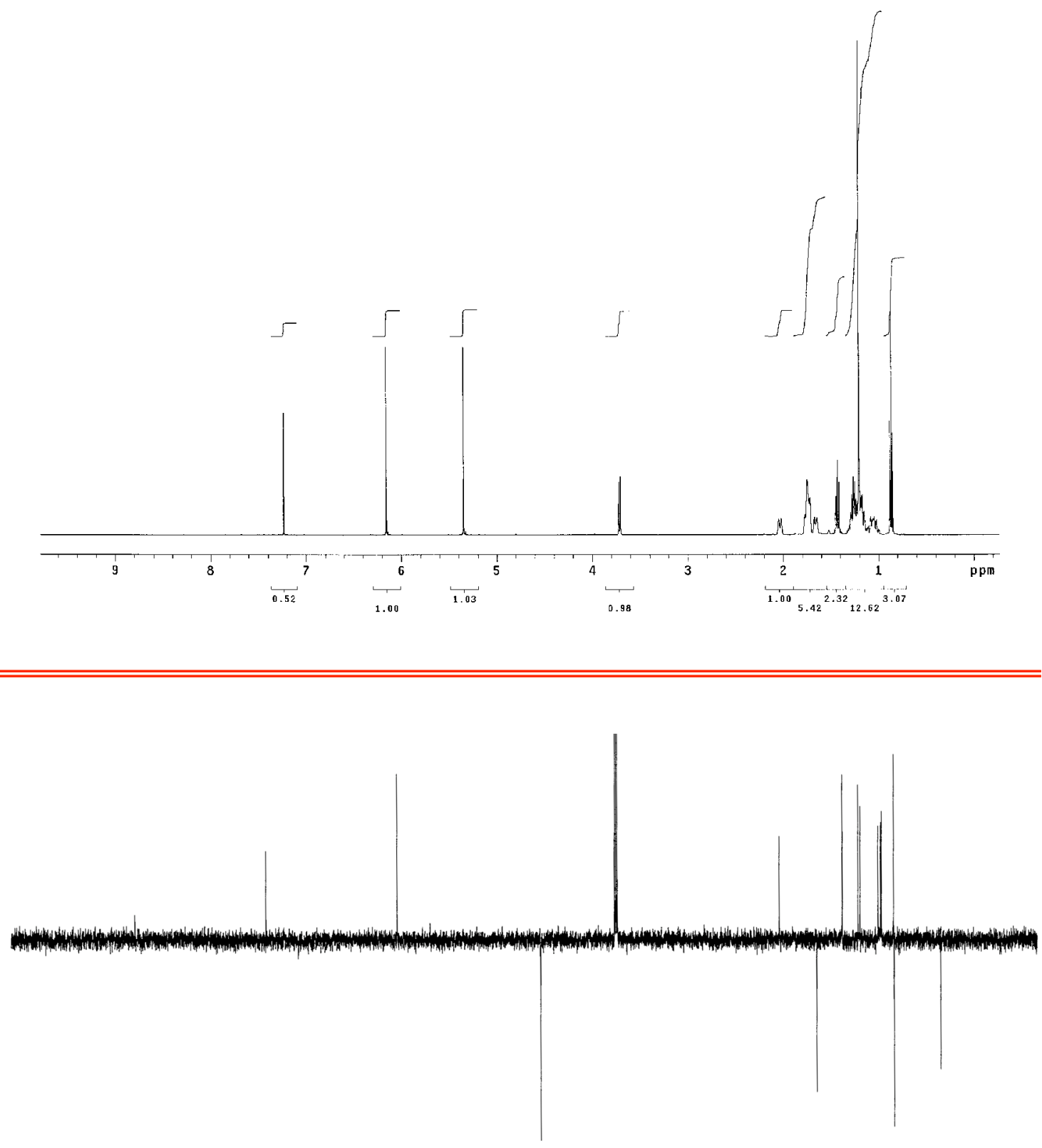

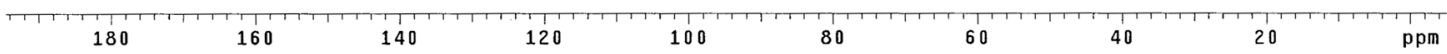


<smiles>C=C1C(=O)OC(C(C)C)[C@@]1(C)Br</smiles>

Lactone 2ag

${ }^{1} \mathrm{H}(500 \mathrm{MHz})$ and ${ }^{13} \mathrm{C}(125 \mathrm{MHz}) \mathrm{NMR}$ in $\mathrm{CDCl}_{3}$
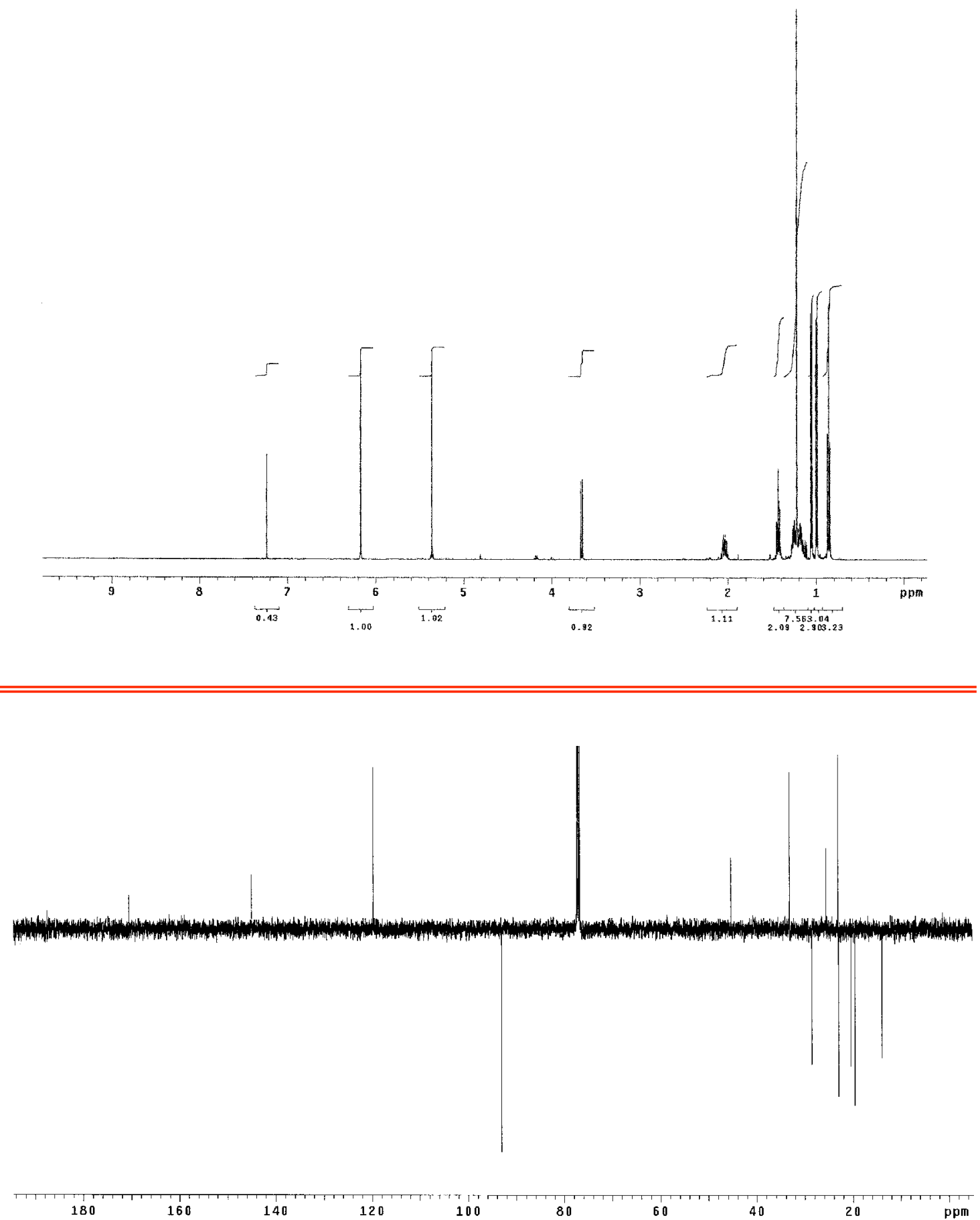


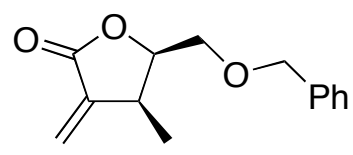

Lactone 2aj

${ }^{1} \mathrm{H}(500 \mathrm{MHz})$ and ${ }^{13} \mathrm{C}(125 \mathrm{MHz}) \mathrm{NMR}$ in $\mathrm{CDCl}_{3}$
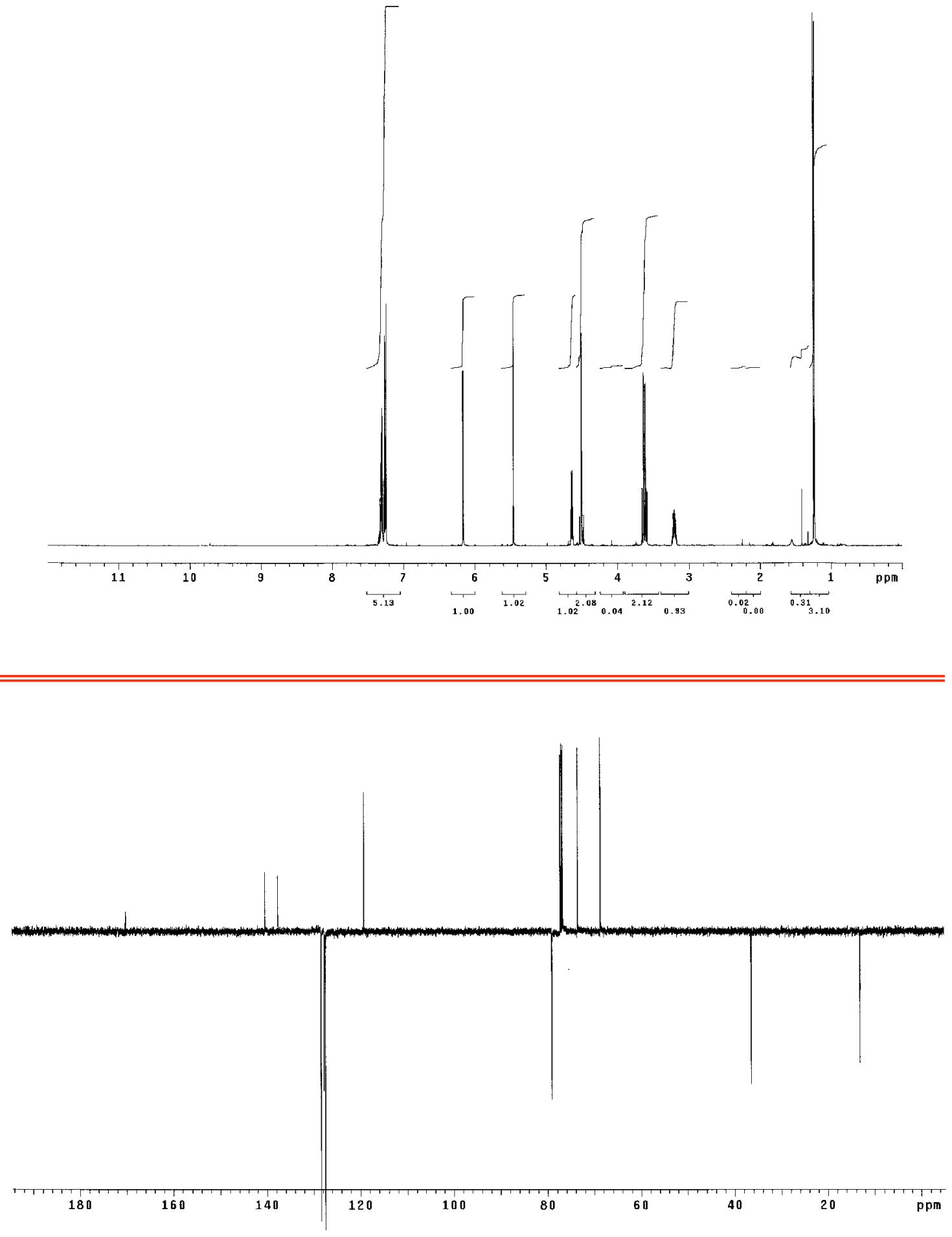
10 NMR spectra of selected single auxiliary chiral alkynoates 10 and allylboronates 11
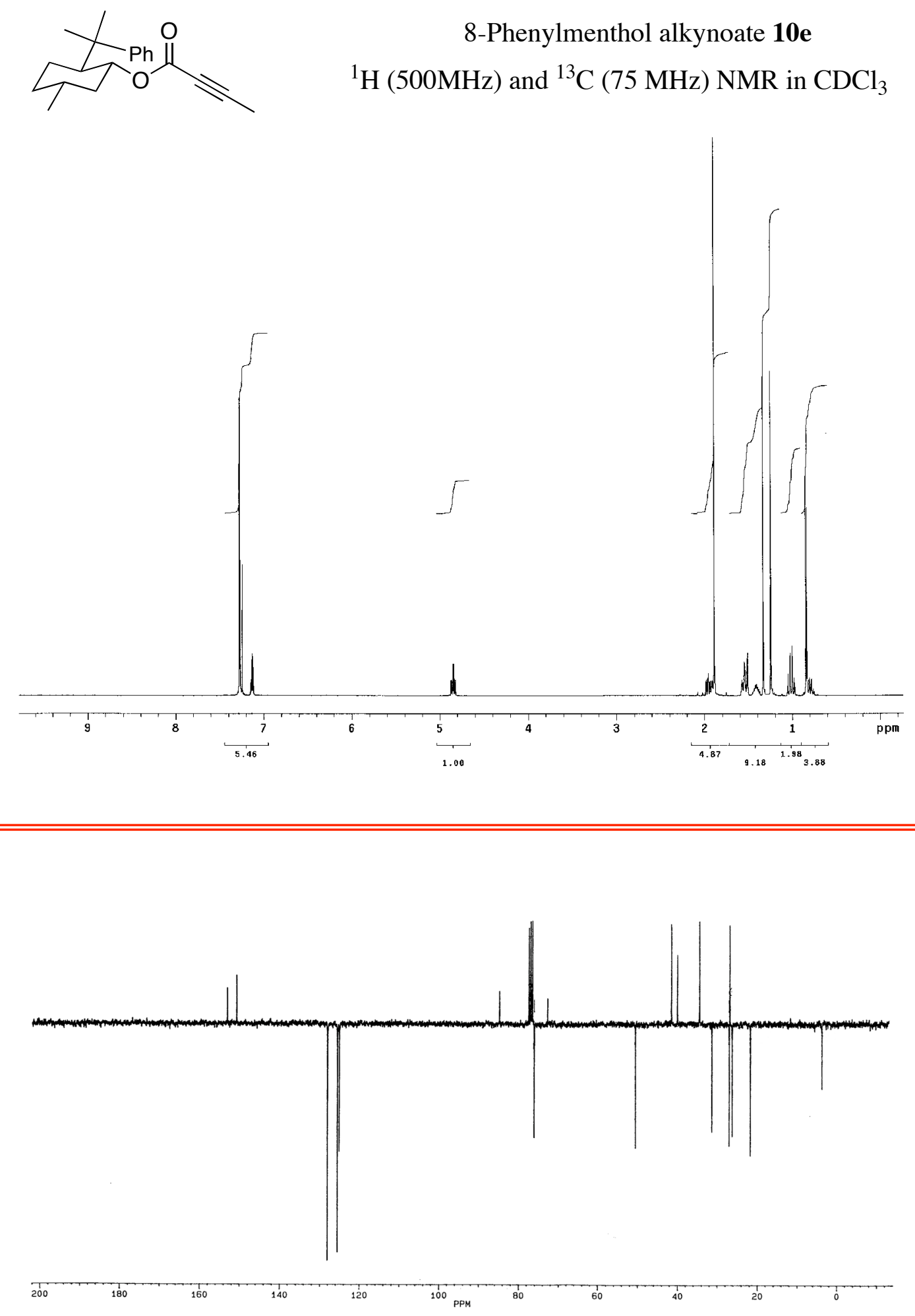


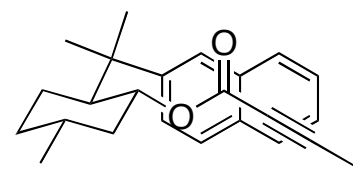

8-(ß-Naphthyl)menthol alkynoate 10f
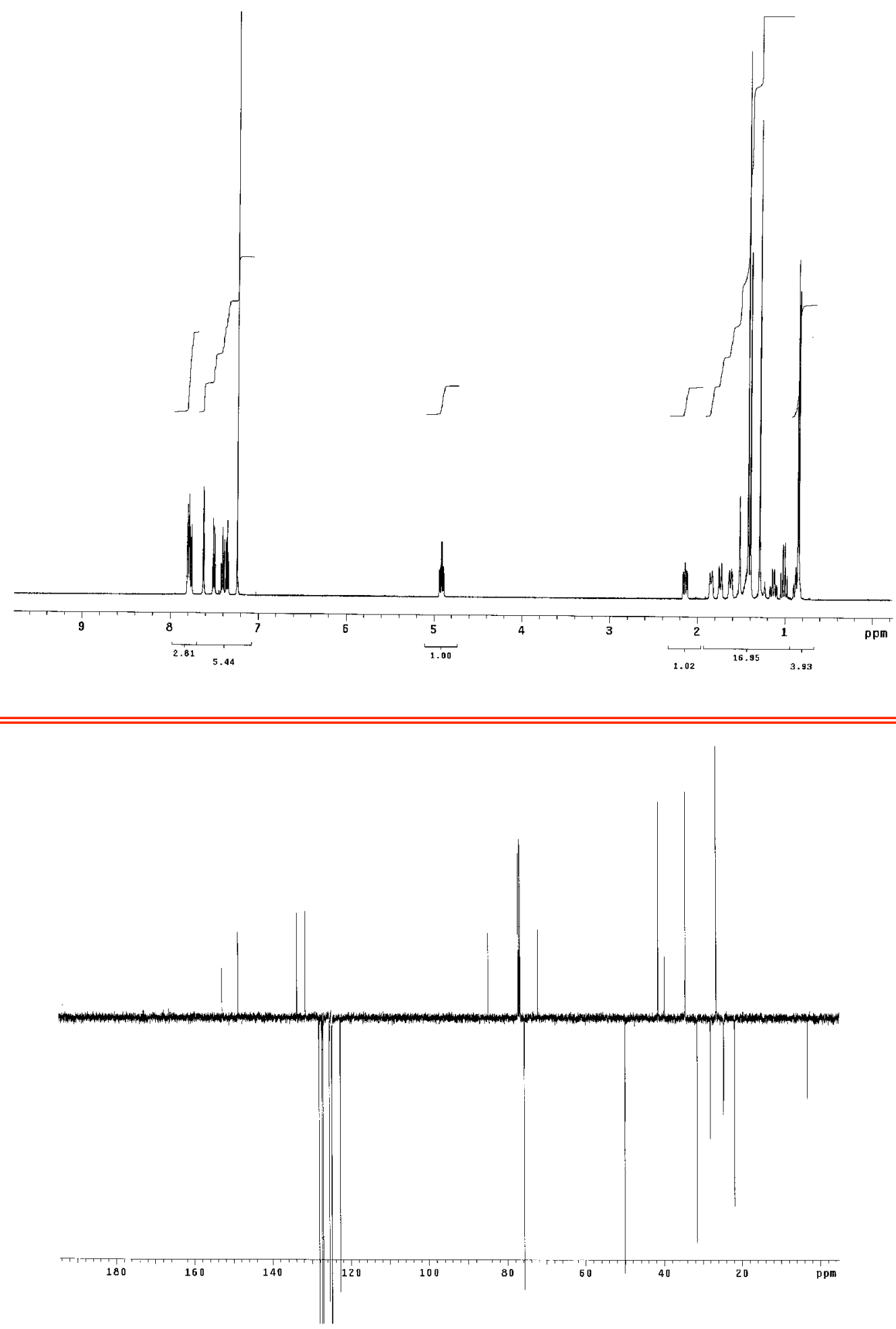


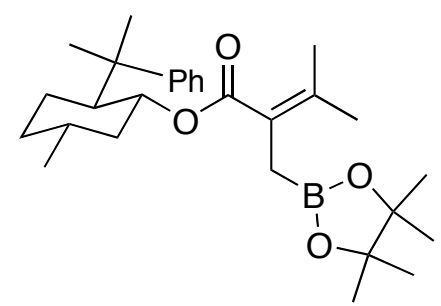

8-Phenylmenthol allylboronate 11e ${ }^{1} \mathrm{H}(400 \mathrm{MHz})$ and ${ }^{13} \mathrm{C}(125 \mathrm{MHz}) \mathrm{NMR}$ in $\mathrm{CDCl}_{3}$
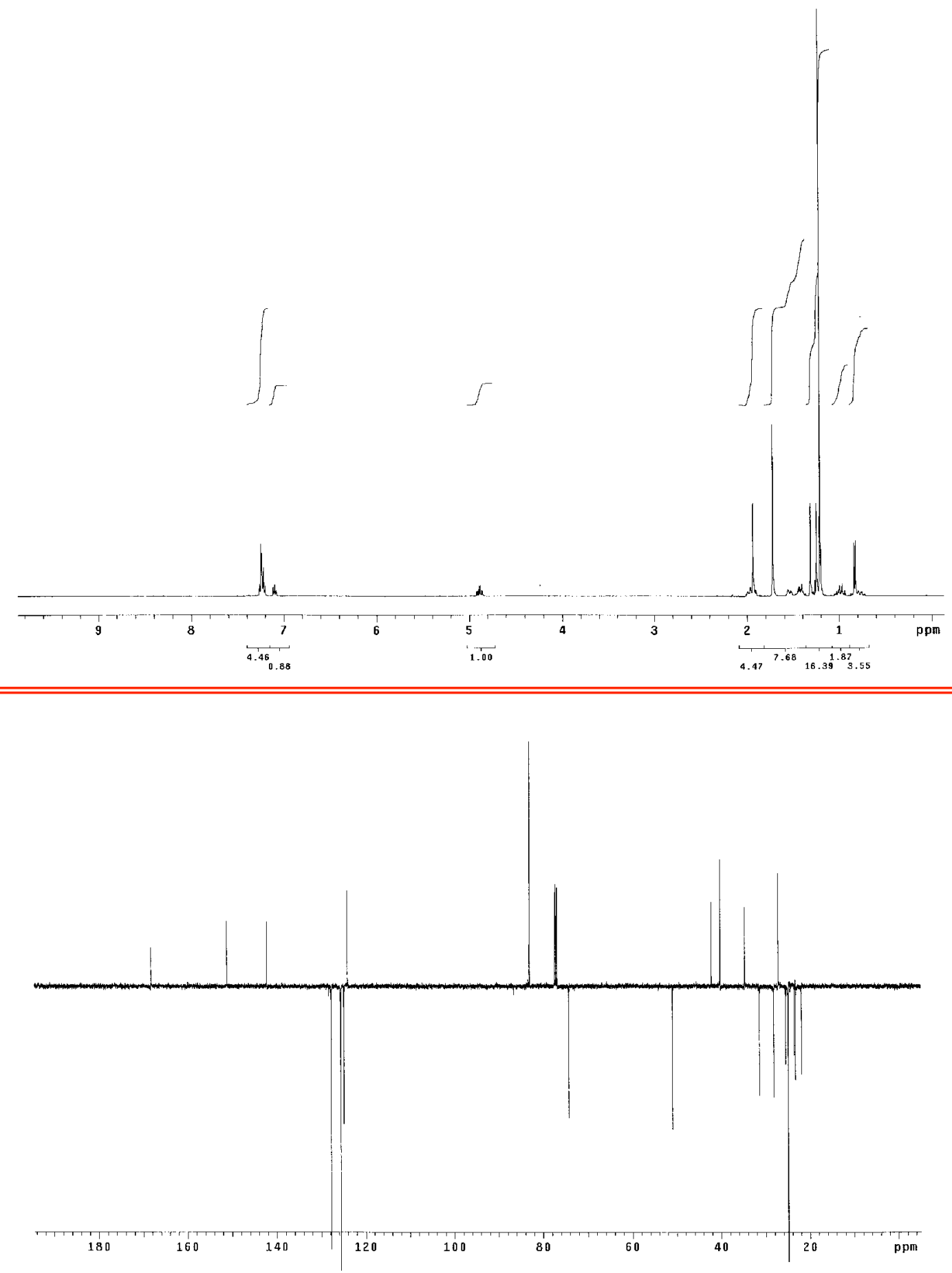


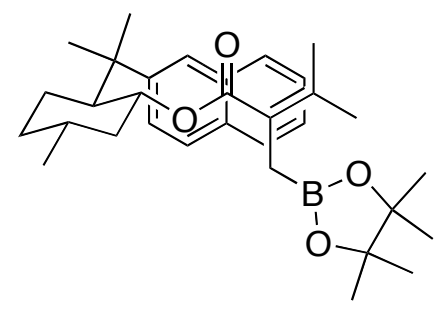

8-(ß-Naphthyl)menthol allylboronate 11f ${ }^{1} \mathrm{H}(500 \mathrm{MHz})$ and ${ }^{13} \mathrm{C}(125 \mathrm{MHz}) \mathrm{NMR}$ in $\mathrm{CDCl}_{3}$
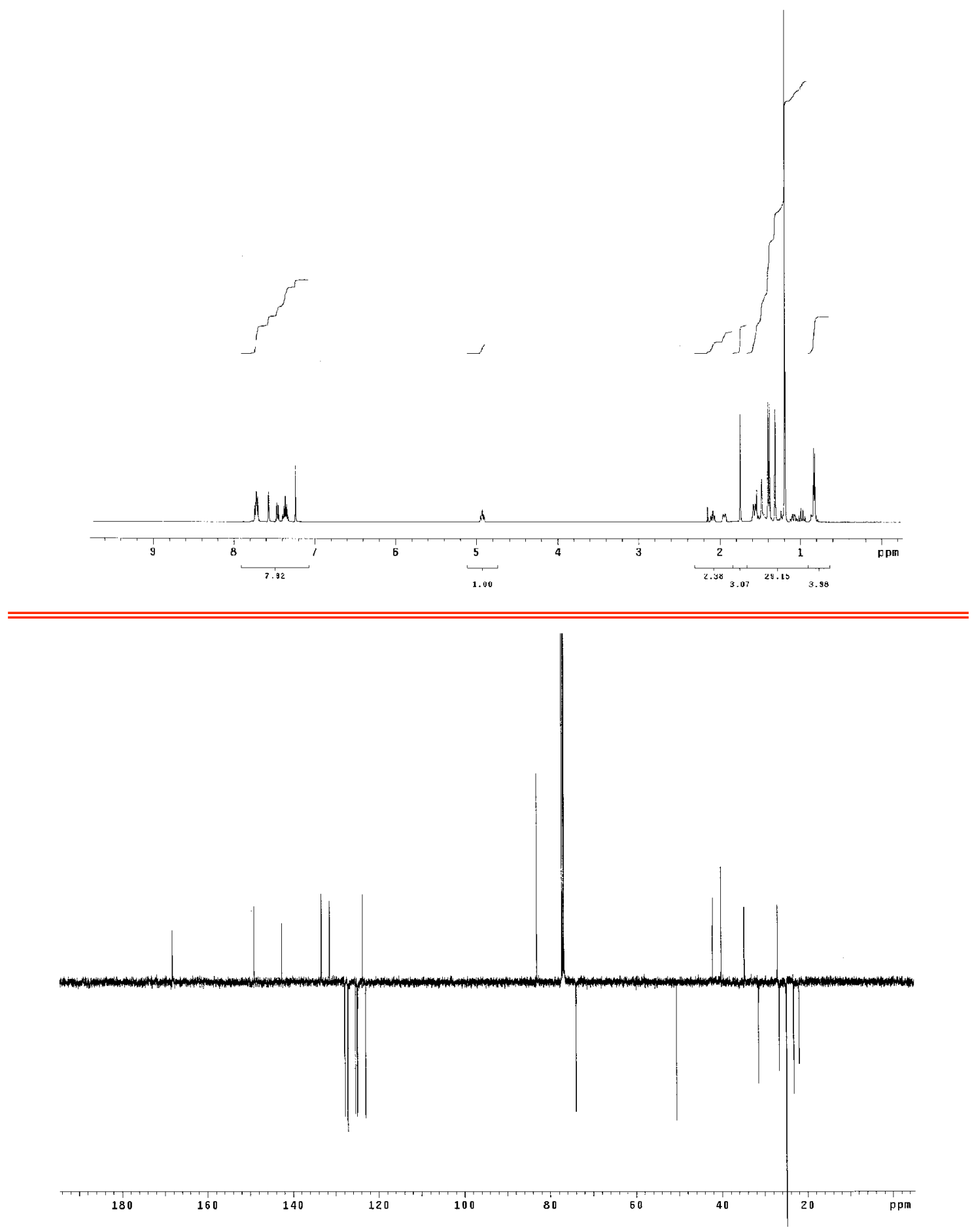


\section{NMR spectra of butyrolactones 27 and diol 28}<smiles>CCC1C(=O)OC(=O)C1C</smiles>

\section{Butyroactone 27a}

${ }^{1} \mathrm{H}(500 \mathrm{MHz})$ and ${ }^{13} \mathrm{C}(125 \mathrm{MHz}) \mathrm{NMR}$ in $\mathrm{CDCl}_{3}$
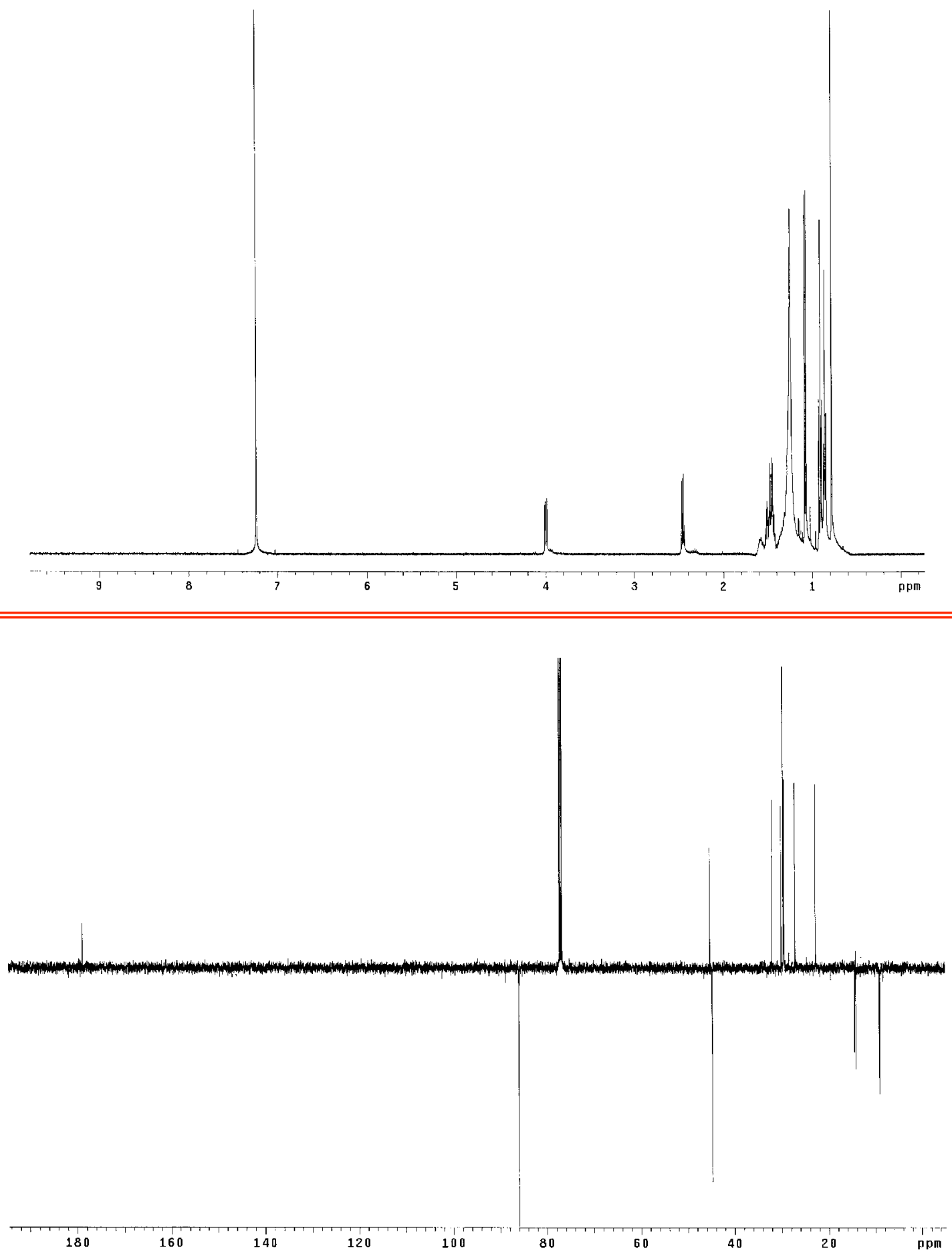


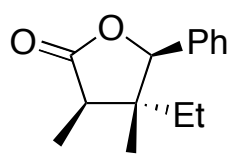

Butyroactone 27b

${ }^{1} \mathrm{H}(400 \mathrm{MHz})$ and ${ }^{13} \mathrm{C}(125 \mathrm{MHz})$ NMR in $\mathrm{CDCl}_{3}$
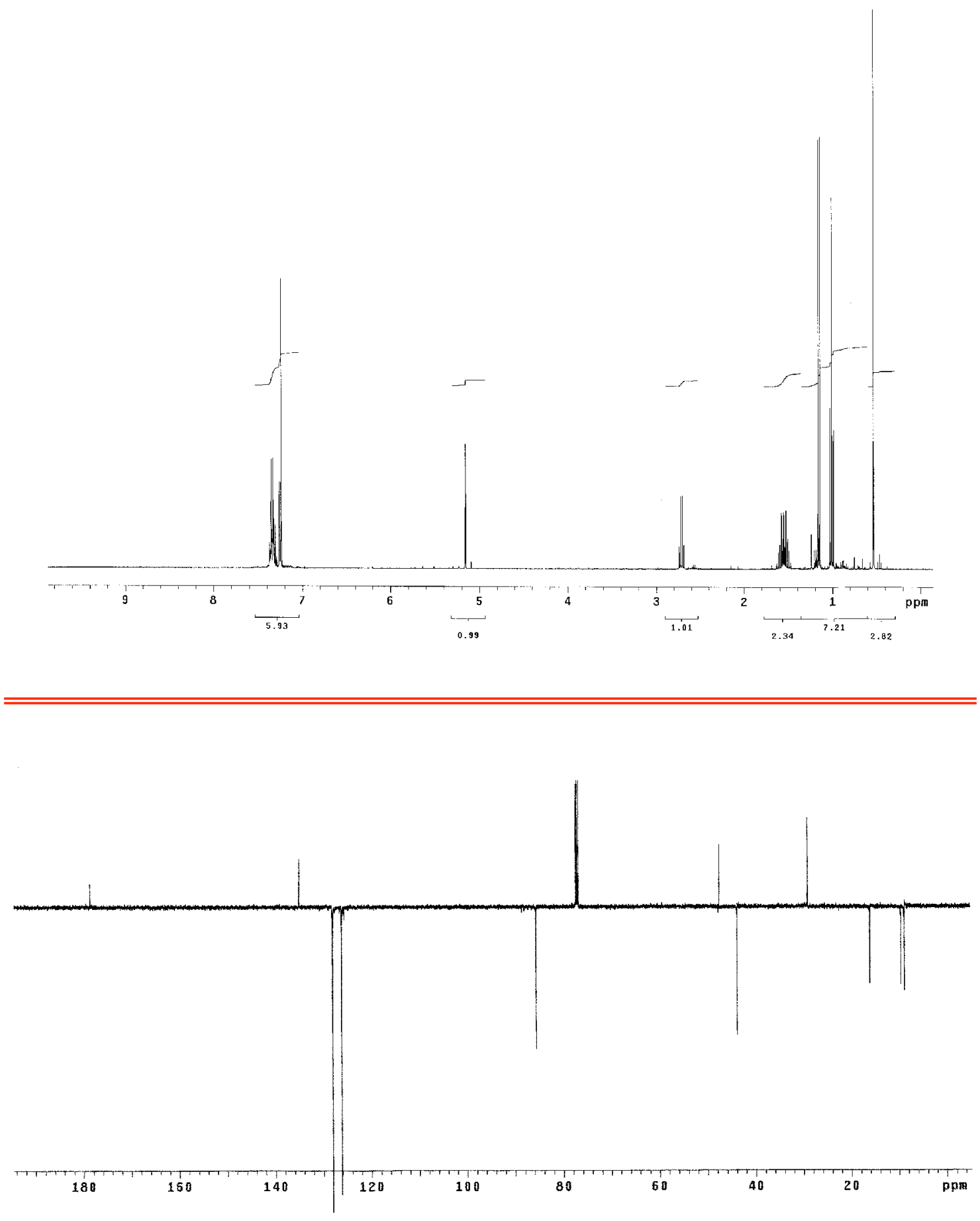


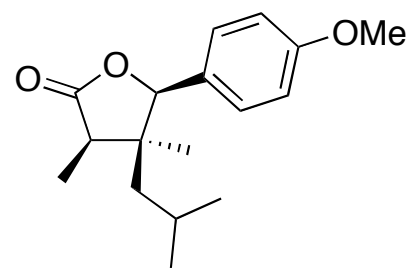

Butyroactone 27c

${ }^{1} \mathrm{H}(500 \mathrm{MHz})$ and ${ }^{13} \mathrm{C}(125 \mathrm{MHz}) \mathrm{NMR}$ in $\mathrm{CDCl}_{3}$
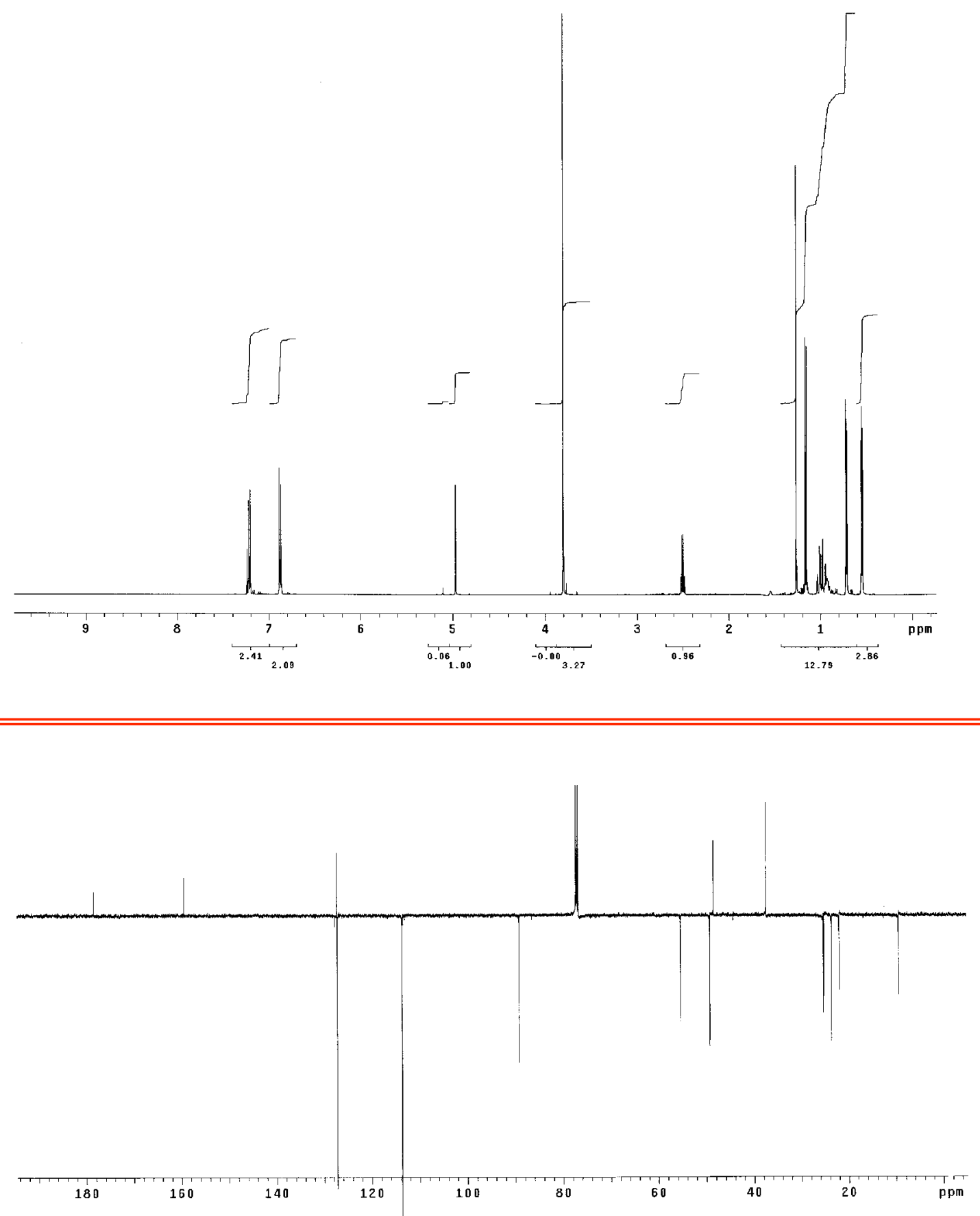

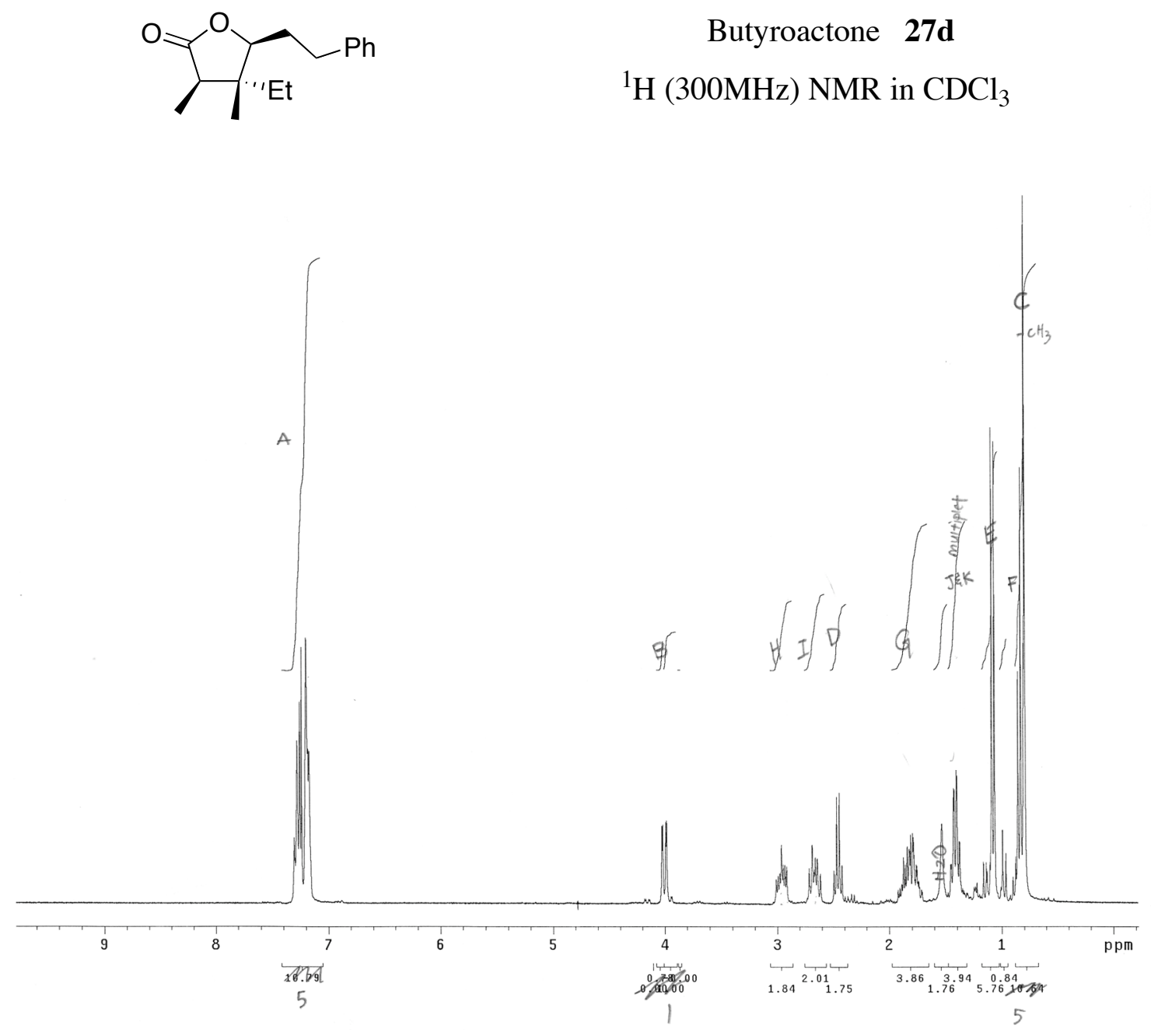
<smiles>CCC(C)(C(C)CO)C(O)C(O)c1ccccc1</smiles>

\section{Diol 28}

${ }^{1} \mathrm{H}(500 \mathrm{MHz})$ and ${ }^{13} \mathrm{C}(125 \mathrm{MHz}) \mathrm{NMR}$ in $\mathrm{CDCl}_{3}$
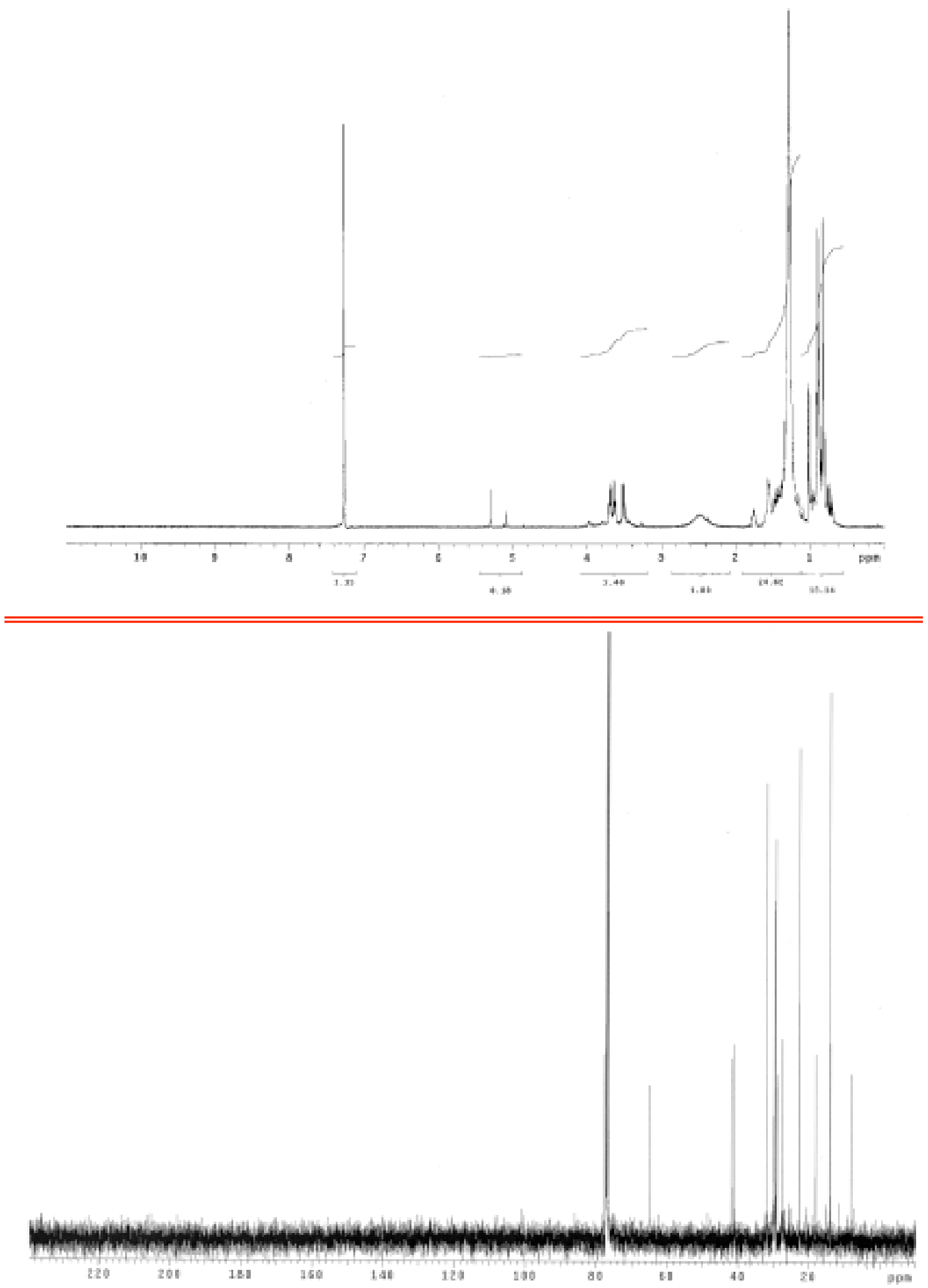